MÉMOIRES DE LA SMF 85

\title{
BOUNDARY COHOMOLOGY OF SHIMURA VARIETIES, III: \\ COHERENT COHOMOLOGY ON \\ HIGHER-RANK BOUNDARY STRATA \\ AND APPLICATIONS \\ TO HODGE THEORY
}

Michael Harris

Steven Zucker

Société Mathématique de France 2001

Publié avec le concours du Centre National de la Recherche Scientifique 


\section{Harris}

Institut de Mathématiques de Jussieu, U.M.R. 7586 du CNRS, Université Paris 7, 2 Pl. Jussieu 75251 Paris cedex 05, France.

E-mail : harris@math.jussieu.fr

Url : http: //www.math. jussieu.fr/ harris

S. Zucker

Department of Mathematics, Johns Hopkins University, Baltimore, MD 21218 USA.

E-mail : sz@math. jhu .edu

Url : http: //www.math. jhu.edu/ ${ }^{\sim}$ sz

2000 Mathematics Subject Classification. - 14G35, 11G18, 14C30, 11F75.

Key words and phrases. - Shimura varieties, automorphic vector bundles, cohomology of arithmetic groups. mixed Hodge structures.

M.H.: Supported in part by the National Science Foundation, through Grant DMS9203142 .

S.Z.: Supported in part by the National Science Foundation, through Grants DMS9423689 and DMS-9820958. 


\title{
BOUNDARY COHOMOLOGY OF SHIMURA VARIETIES, III: COHERENT COHOMOLOGY ON HIGHER-RANK BOUNDARY STRATA AND APPLICATIONS TO HODGE THEORY
}

\author{
Michael Harris, Steven Zucker
}

\begin{abstract}
In this article, third of a series, we complete the verification of the following fact. The nerve spectral sequence for the cohomology of the Borel-Serre boundary of a Shimura variety Sh is a spectral sequence of mixed Hodge-de Rham structures over the field of definition of its canonical model. To achieve that, we develop the machinery of automorphic vector bundles on mixed Shimura varieties, for the latter enter in the boundary of the toroidal compactifications of Sh; and study the nerve spectral sequence for the automorphic vector bundles and the toroidal boundary. We also extend the technique of averting issues of base-change by taking cohomology with growth conditions. We give and apply formulas for the Hodge gradation of the cohomology of both Sh and its Borel-Serre boundary.
\end{abstract}

Résumé (Cohomologie au bord des variétés de Shimura, III). - Dans cet article, troisième d'une série, nous terminons la vérification du fait suivant. La suite spectrale « du nerf », qui calcule la cohomologie du bord de la compactification de Borel-Serre d'une variété de Shimura Sh, est une suite spectrale de structures de Hodge-de Rham mixtes sur le corps de définition de son modèle canonique. Pour le faire, nous développons la théorie de fibrés automorphes sur les variétés de Shimura mixtes, car de tels objets figurent dans le bord d'une compactification toroïdale de Sh; et nous étudions la suite spectrale «du nerf » pour les fibrés automorphes et le bord toroïdal. En plus, nous généralisons nos résultats antérieurs sur la cohomologie avec conditions de croissance, qui permettent d'éviter les difficultés associées au changement de base. Enfin, nous énonçons et appliquons des formules pour la graduation de Hodge de la cohomologie de Sh et celle du bord de sa compactification de Borel-Serre. 



\section{CONTENTS}

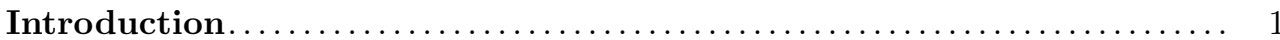

1. Automorphic vector bundles on mixed Shimura varieties......... 9

1.1. Mixed Shimura varieties.................................... 9

1.2. Construction of automorphic vector bundles.................... 14

1.3. Canonical extensions of automorphic vector bundles............... 23

1.4. Functorial properties of canonical extensions ..................... 28

1.5. A boundary calculation $\ldots \ldots \ldots \ldots \ldots \ldots \ldots \ldots \ldots \ldots \ldots \ldots \ldots \ldots \ldots \ldots \ldots \ldots$

2. Mixed growth conditions and coherent cohomology ............. 41

2.1. $\bar{\partial}$ with logarithmic growth in one variable (cf. [HP], [H4, § 3], [HZ1, 3.8]) . 41

2.2. Logarithmic growth in several variables........................ 43

2.3. Forms with mixed growth conditions $\ldots \ldots \ldots \ldots \ldots \ldots \ldots \ldots \ldots \ldots, \ldots 4$

2.4. Siegel sets in mixed Shimura varieties........................ 44

2.5. Toroidal compactifications and Siegel sets.................... 46

2.6. Growth conditions at the toroidal boundary .................... 49

2.7. Mixed growth conditions and basechange $\ldots \ldots \ldots \ldots \ldots \ldots \ldots \ldots \ldots . \ldots \ldots$

3. The nerve spectral sequence for coherent cohomology ........... 55

3.1. Cohomology on boundary strata of toroidal compactifications.......... 55

3.2 . The nerve spectral sequence for boundary cohomology $\ldots \ldots \ldots \ldots \ldots \ldots 61$

3.3. Differentials in the nerve spectral sequence...................... 63

3.4. Differentials in the nerve spectral sequence, concluded.............. 65

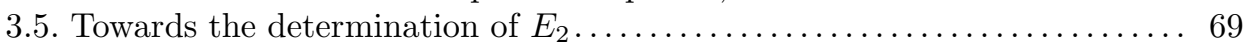

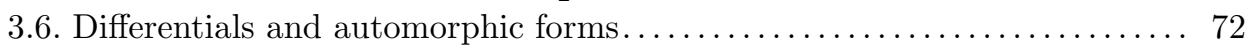




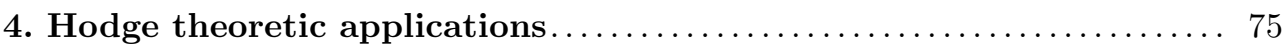

4.1. The Hodge filtration for boundary cohomology . . . . . . . . . . . . . . 75

4.2. The dual Bernstein-Gelfand-Gelfand complex................. 80

4.3. Hodge theory at the boundary, revisited $\ldots \ldots \ldots \ldots \ldots \ldots \ldots \ldots \ldots \ldots . \ldots \ldots$

4.4. The topological cohomology ............................. 95

4.5. The mixed Hodge complex filtered by holomorphic rank.............. 100

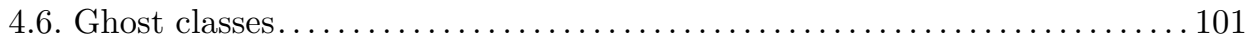

5. On the comparison of Hodge structures.................... 109

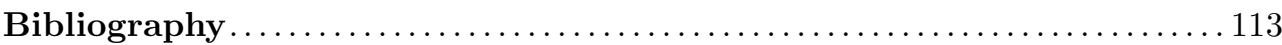




\section{INTRODUCTION}

The present article continues the study of the boundary cohomology of Shimura varieties initiated in [HZ1, HZ2]. Let $G$ be a reductive group over $\mathbb{Q}, X$ the symmetric space associated to $G(\mathbb{R})$, and $\Gamma$ a congruence subgroup of $G(\mathbb{Q})$. We consider the cohomology of $\Gamma \backslash X$ with coefficients in the local system $\widetilde{\boldsymbol{V}}$ constructed from a representation $V$ of $G$, i.e., $H^{\bullet}(\Gamma \backslash X, \widetilde{\boldsymbol{V}}) \simeq H^{\bullet}(\Gamma, V)$. It is standard that this cohomology can be decomposed as the direct sum of "interior" cohomology, defined as the image of the cohomology with compact supports $H_{c}^{\bullet}(\Gamma \backslash X, \widetilde{\boldsymbol{V}})$, and a complementary "boundary cohomology" that restricts non-trivially to the boundary of the Borel-Serre (manifold-with-corners) compactification of $\Gamma \backslash X$. The designation of boundary cohomology is generally non-canonical, and much work has been devoted to constructing canonical decompositions using Eisenstein series.

By an elaboration on the de Rham theorem, one knows that the cohomology group $H \bullet(\Gamma \backslash X, \widetilde{\boldsymbol{V}})$ can be expressed as the relative Lie algebra cohomology of the space of $V$ valued $C^{\infty}$ functions on $\Gamma \backslash G(\mathbb{R})$, or even the functions of moderate growth ([B2, $\left.\S 7\right]$ ). Thanks to the work of Franke [Fr1], one can replace the functions of moderate growth by the subspace of automorphic forms, and this can provide the starting point for an approach to the boundary cohomology. However, in this series of articles we are concerned only tangentially with the relation between boundary cohomology and automorphic forms. We choose to work at a more intrinsic level, concentrating instead on the additional structures on $H \bullet(\Gamma \backslash X, \widetilde{\boldsymbol{V}})$ when $X$ is a hermitian symmetric domain. In that case, $\Gamma \backslash X$ is an algebraic variety, and $\widetilde{\boldsymbol{V}}$ underlies a natural variation of Hodge structure. Morihiko Saito's theory of mixed Hodge modules [Sa3] then gives that $H \bullet(\Gamma \backslash X, \widetilde{\boldsymbol{V}})$ has a corresponding mixed Hodge structure (MHS). The nature of this MHS at the boundary - more accurately, the associated MHS on the deleted neighborhood cohomology of the boundary - was the subject of [HZ2].

The "adelic version" of $\Gamma \backslash X$ is the Shimura variety $\operatorname{Sh}(G, X)$, whose connected components are of the form $\Gamma \backslash X$. This has a canonical model over a number field $E$. The de Rham isomorphism identifies $H \bullet(\operatorname{Sh}(G, X), \widetilde{\boldsymbol{V}})$ with the hypercohomology of of an $E$-rational complex of coherent sheaves on $\operatorname{Sh}(G, X)$; thus $H^{\bullet}(\operatorname{Sh}(G, X), \widetilde{\boldsymbol{V}})$ 
acquires an $E$-rational structure distinct from the topological rational structure coming from the coefficients $\widetilde{\boldsymbol{V}}$. In particular, $H^{\bullet}(\operatorname{Sh}(G, X), \widetilde{\boldsymbol{V}})$ has a Hodge filtration whose graded pieces are given by the coherent cohomology with coefficients in certain automorphic vector bundles [H1, Mi2]; the latter have natural E-rational structure. (This $E$-rationality can be asserted for de Rham cohomology, without grading for the Hodge filtration, and that is conjecturally equivalent in this context.) Study of the boundary cohomology of such automorphic vector bundles was begun in [HZ1].

Both [HZ1] and [HZ2] made essential use of toroidal compactifications of Shimura varieties (which has its origin in $[\mathrm{AMRT}]$ ), following [H3] and [H4]. The toroidal boundary of $\operatorname{Sh}(G, X)$ (a divisor with normal crossings), like the Borel-Serre boundary, is stratified according to conjugacy classes of parabolic subgroups of $G$. The cohomology of the boundary, both in the topological setting (as above) and in the coherent setting (i.e., for canonically extended automorphic vector bundles), can be computed as the abutment of the spectral sequence for the closed covering given by this stratification; this is called the nerve spectral sequence. In [HZ1] we analyzed the contribution to the nerve spectral sequence from the strata associated to maximal parabolics in the coherent setting. The first task of the present article is to extend this analysis to general parabolics, thereby fulfilling our promise from [HZ1], and this is carried out in the first three Chapters. This necessitated a generalization in Chapter 1 of much of the machinery of automorphic vector bundles to the toroidal compactifications of mixed Shimura varieties (constructed by Pink $[\mathrm{P}]$ ).

Most of the calculations from $[\mathrm{HZ} 1, \S 3]$ go over without change, but there are a few delicate points, notably the issue of basechange in (3.4). For the latter, we must recall the role of conditions of growth and decay. These entered in the coherent setting when we established the existence and degeneration of Leray spectral sequences for morphisms of toroidally compactified varieties and the corresponding morphisms of canonical (or subcanonical) extensions of automorphic vector bundles. In effect, it enabled us to circumvent the complications related to basechange at infinity. As suggested above, this last point recurs here. We are obliged to prove (in (2.3)) a generalization to mixed conditions of growth and decay, enabling us, in effect, to isolate a single boundary stratum.

Our main result in Chapter 3 is that the differentials in the $E_{1}$-term of the nerve spectral sequence for coherent cohomology decompose naturally into pieces that either are given in terms of restriction maps on pure Shimura varieties or are "purely topological" (see (3.5.4)). Via Franke's interpretation of cohomology in terms of automorphic forms, this implies (see (3.6)) that the constant term maps for cohomology, expressed as integration of an automorphic form along the unipotent radical of appropriate parabolic subgroups, are rational with respect to the de Rham rational structure; for maximal parabolics, this was already obtained in [HZ1, 4.8]. 
The nerve spectral sequence for the topological cohomology $H \bullet(\Gamma \backslash X, \widetilde{\boldsymbol{V}})$ was studied in detail in [HZ2]. Hodge-theoretic considerations require (algebraic) compactifications, and the toroidal compactifications were convenient to use for this purpose as well. It was a subtle matter to compare the deleted neighborhoods of the BorelSerre and toroidal boundary strata associated to a given parabolic subgroup (see $[\mathrm{HZ} 2, \S 2])$. We constructed isomorphisms between them that are compatible with restriction maps, allowing for transport of structure from the latter to the former. From this, it follows that the differentials in the topological nerve spectral sequence are morphisms of mixed Hodge structures. In particular, they induce maps after grading for the Hodge filtration $F$. Since a morphism of mixed Hodge structures is determined by its gradation for $F$, it follows, for instance, that ghost classes exist in $H^{\bullet}(\Gamma \backslash X, \widetilde{\boldsymbol{V}})$ if and only if they exist in $\operatorname{Gr}_{F} H \bullet(\Gamma \backslash X, \widetilde{\boldsymbol{V}}$ ) (see (4.6.7)). (Recall that a ghost class in $H^{\bullet}(\Gamma \backslash X, \widetilde{\boldsymbol{V}})$ is a cohomology class whose restriction to the Borel-Serre boundary is non-zero, yet whose restriction to each face (stratum) thereof is zero.) In (4.1), we compare the graded differentials to the results obtained for the differentials in the case of the coherent cohomology. To that end, we derive a formula for the deleted neighborhood cohomology of a boundary stratum as de Rham cohomology on a suitable toroidal compactification of the associated (Baily-Borel) boundary component (see (4.1.9)).

Of course, the above can be repeated for the weight filtration. For an example of the use of weights to rule out ghost classes (cf. (4.6.14)), see [Z5, App. A].(1) We are still seeking a satisfactory way of dealing with the entire mixed Hodge structure. It is therefore strongly to be feared that this article is not the last of the series ... The content of the first three chapters of this article completes the verification of results announced in $[\mathrm{HZ} 1, \S 5]$ and in $[\mathrm{H} 5]$. They can be summarized by saying that the (topological) nerve spectral sequence is a spectral sequence of mixed Hodge-de Rham structures over the field of definition of the canonical model.

In Chapter 4, we continue to develop the Hodge theoretic material from [HZ2, §5]. In (4.2), we reformulate the results in (4.1) by using the "minimal model" of the holomorphic de Rham complex, viz., the dual Bernstein-Gelfand-Gelfand complex, and deduce the $E$-rational version of (4.1.9).

A big surprise in this work was the discovery of another interesting filtration on the boundary complex, whose spectral sequence is, like the nerve spectral sequence, a spectral sequence of mixed Hodge structures. In a way, there is nothing new about this filtration, which we call the filtration by holomorphic rank; it is given by the pullback to the Borel-Serre boundary of the filtration of the Baily-Borel Satake boundary by

\footnotetext{
${ }^{(1)}$ The correct outcome of the calculation presented in the latter is that there are no ghosts for $G S p(4)$ when the representation $V$ is generic, i.e., where the highest weight for $S p(4)$ has positive inner product with both simple roots. When $V$ is trivial, on the other hand, the calculation does allow for a weight-two ghost class in $H^{2}(\Gamma \backslash X, \mathbb{Q})$, and such a class is determined in [KR, 14.1.3].
} 
(unions of) boundary strata of increasing dimension (see also (4.4.15)). In a sense that can be made precise, its $E_{1}$-term is closer to the abutment than that of the nerve spectral sequence, though further from the question of ghost classes. We treat the holomorphic rank filtration in (4.4), though the same considerations already show up in (3.5) in the coherent setting. Cases of the latter give the Hodge components for the $E_{1}$-term of the topological holomorphic rank spectral sequence, and this gets examined in (4.5).

Several fundamental questions remain open. The analysis of cohomology of Shimura varieties should be extended to the intersection cohomology of their minimal (BailyBorel) compactifications. The Zucker conjecture, proved by Looijenga $[\mathrm{L}]$ and SaperStern [SS], asserts that this cohomology is isomorphic to the $L_{2}$-cohomology, or again to the Lie algebra cohomology of square-integrable $C^{\infty}$ functions, or by [Fr1], of square-integrable automorphic forms. However, it is not known whether this isomorphism identifies Morihiko Saito's Hodge structure with the analytic Hodge structure on $L_{2}$-cohomology (the one given a priori by the $L^{2}$ harmonic forms). In Chapter 5 we obtain a partial result in this direction: we show that the map from $L_{2}$-cohomology of the open Shimura variety to ordinary cohomology is a morphism of (mixed) Hodge structures (this is a small improvement over what was asserted in $[\mathrm{H} 5,3.3 .9]$ ). We do not address the question of whether intersection cohomology carries a de Rham rational structure.

It is also true that not all questions are treated in maximum generality. For instance, we have not studied the cohomology of a general automorphic vector bundle or variation of mixed Hodge structure on a mixed Shimura variety, but have rather been content to work out the cases directly relevant to the cohomology of pure Shimura varieties. Experience suggests these omissions will return to haunt us (providing even more impetus for article IV?). Another thing absent is the exploration of relations between our constructions and the general polylogarithms constructed by Wildeshaus [W1, W2].

Much of this work was begun at the time of writing of [H5], where some of our results were announced. The actual writing of the present article did not get under way until the second-named author visited Université Paris 7 in May, 1997. We both wish to thank that institution for the hospitality extended on that occasion. Likewise, a large amount of the work and writing of this article was carried out while the second-named author was spending Academic Year 1998-99 on sabbatical at the Institute for Advanced Study in Princeton. We also wish to thank P. Polo for helpful discussions of the generalized Bernstein-Bernstein-Gelfand resolution, and Z. Mebkhout for help with the proof of Proposition (4.2.21). We thank J. Wildeshaus for numerous thoughtful comments on both the content and the exposition of the article. Finally, we are grateful to the referee for his careful reading of the first 
version of the present article and for his numerous suggestions, which we believe have led to a substantial improvement of the first section, in particular.

\section{Conventions and notation}

0.1. We let $\underline{S}=R_{\mathbb{C} / \mathbb{R}} \mathbb{G}_{m, \mathbb{C}}$ be the torus whose real representations parametrize $\mathbb{R}$ Hodge structures. To any real representation $h: \underline{S} \rightarrow G L(V)$ is associated a Hodge structure on $V$ such that $V^{p, q}$ is the subspace of $V_{\mathbb{C}}$ on which $z \in \underline{S}(\mathbb{R}) \stackrel{\sim}{\longrightarrow} \mathbb{C}^{\times}$acts as $z^{-p} \bar{z}^{-q}$. The Hodge structure thus obtained is canonically the direct sum of its weight graded components. We let $\mu: \mathbb{G}_{m, \mathbb{C}} \rightarrow \underline{S}_{\mathbb{C}}$ be the cocharacter such that $h \circ \mu(z)$ acts as $z^{-p}$ on $V^{p, q}$, for any $h$ and $V$ as above. We let $w: \mathbb{G}_{m, \mathbb{R}} \rightarrow \underline{S}$ be the natural map. Then with $(h, V)$ as above, the associated Hodge structure is pure of weight $w(V)$ if and only if $h \circ w$ defines a character of $\mathbb{G}_{m, \mathbb{R}}$, and in that case $h \circ w(t)=t^{-w(V)}$.

0.2. Let $G$ be a connected reductive group over $\mathbb{C}, P \subset G$ a parabolic subgroup, $L \subset P$ a Levi subgroup, $H \subset L$ a maximal torus, $R_{u} P \subset P$ the unipotent radical. Let $B \subset P$ be a Borel subgroup of $G$ containing $H$. We use the lower case Gothic letters $\mathfrak{g}, \mathfrak{p}, \mathfrak{h}, \mathfrak{l}, \mathfrak{b}$ to denote the corresponding Lie algebras, and let $\Phi=\Phi(\mathfrak{g}, \mathfrak{h})$ be the set of roots, $\Phi^{+}$the positive roots corresponding to $\mathfrak{b}, \Phi_{L}=\Phi(\mathfrak{l}, \mathfrak{h})$ and $\Phi_{L}^{+}$ respectively the roots and positive roots of $\mathfrak{h}$ in $\mathfrak{l}$. Let $W=W(\mathfrak{g}, \mathfrak{h})$ be the Weyl group, $W_{L}=W(\mathfrak{l}, \mathfrak{h})$. The subset $W^{P} \subset W$ of Kostant representatives of $W_{L} \backslash W$ is the set

$$
\left\{w \in W \mid w^{-1}(\alpha)>0, \forall \alpha \in \Phi_{L}^{+}\right\} .
$$

Then $W^{P}$ is the set of representatives of shortest length for the right cosets $W_{L} \backslash W$.

The same terminology is used for the shortest representatives of $W_{L}$ in $W$ when $G$ is quasi-split over a field of characteristic zero.

0.3. Starting in $\S 3$ we will concentrate on the case of pure Shimura varieties. Notation is as in [HZ1] and [HZ2] (see, however, $(0.5)$ ). In particular, $(G, X)$ is a Shimura datum, $K_{p} \subset G_{\mathbb{R}}$ is the (algebraic) identity component of the stabilizer of a point $p \in X, H \subset K_{p}$ is a maximal torus, $\mathfrak{h}$ its Lie algebra. We assume a set of positive roots $\Phi^{+}$chosen as above, with decomposition $\Phi^{+}=\Phi_{c}^{+} \cup \Phi_{n}^{+}$into compact and non-compact roots, as in [HZ1,3.6] (where we used the letter $R$ instead of $\Phi$ ). All references to highest weights will be with respect to these choices. Let $\mathfrak{P}^{+} \subset \mathfrak{g}$ (resp. $\mathfrak{P}^{-}$) be the maximal parabolic subalgebra with Levi component $\mathfrak{k}_{p}$ and unipotent radical $\mathfrak{p}^{+}\left(\right.$resp. $\left.\mathfrak{p}^{-}\right)$.

0.4. We wish to recall the combinatorics of the intersection of boundary strata in the Borel-Serre and toroidal compactifications of the locally symmetric variety $\Gamma \backslash X$. This goes the same for both, so we will treat only the former.

The Borel-Serre compactification is $\Gamma \backslash \bar{X}$, where $\bar{X}$ is a certain manifold-withcorners with $G(\mathbb{Q}$ )-action, with $X$ as interior. Its closed (or open) boundary faces are in natural one-to-one correspondence with the rational parabolic subgroups $P$ of $G$, 
and are denoted $\overline{e(P)}$. Then $\overline{e(P)} \cap \overline{e(Q)} \neq \varnothing$ if and only if $P \cap Q$ is parabolic, and then the intersection is simply $\overline{e(P \cap Q)}$.

In $\Gamma \backslash \bar{X}$, one has boundary faces indexed by $\Gamma$-conjugacy classes of rational parabolic subgroups. It is customary to take representatives of these conjugacy classes, so one must understand the notational complication that ensues. The boundary face $\overline{e^{\prime}(P)}$ of $\Gamma \backslash \bar{X}$ is an arithmetic quotient of $\overline{e(P)}$, so likewise for any $\Gamma$-conjugate of $P$. Thus, it is wrong to expect that $\overline{e^{\prime}(P)} \cap \overline{e^{\prime}(Q)}=\overline{e^{\prime}(P \cap Q)}$ when $P \cap Q$ is parabolic. Indeed, $\overline{e^{\prime}(P \cap Q)}$ is only one connected component of the intersection. Suppose that $P \cap Q$ is the parabolic subgroup $R$. Then $\overline{e^{\prime}(P)} \cap \overline{e^{\prime}(Q)}$ is a finite disjoint union of faces of the form $\overline{e^{\prime}\left(g R g^{-1}\right)}$, for $g$ running over representatives of the fiber over the identity double coset in the right-hand side of

$$
(\Gamma \cap P) \backslash P(\mathbb{Q}) / R(\mathbb{Q}) \longrightarrow \Gamma \backslash G(\mathbb{Q}) / Q(\mathbb{Q}) .
$$

This understanding underlies all treatment of intersections of boundary strata in this work. (For more on this, see the appendix to [HZ2, (3.5)].)

0.5. We wish to remind the reader that it was necessary in $[\mathrm{HZ2},(1.4)]$ to make a change of notation for the sequel, in particular this paper. In [HZ1], we used the symbol $F$ to denote a boundary component, and associated objects such as its normalizing parabolic subgroup $P_{F}$, the Cayley transform $c_{F}$, the associated cone complex $\Sigma_{F}$, etc. carried the subscript $F$. Given that $P=P_{F}$ and $F$ are equivalent data, we decided it was more convenient to switch to writing $P, c_{P}, \Sigma_{P}$, etc. As such, one must take this change into account when tracking down notions from [HZ1]. For example, the Shimura datum denoted $\left(G_{h, F}, X(F)\right)$ in $[\mathrm{HZ1} 1.1 .7]$ is here denoted $\left(G_{h, P}, X(P)\right)$

The notation $Z_{\Sigma}(R)$ (for the closed $R$-stratum of the toroidal boundary) was introduced in [HZ2, (1.5.2)]. It derives from [HZ2, (1.4.11)]; there, the $F$-stratum should have been denoted $<Z_{F, \Sigma}$ (as in 1.5.2 of [HZ1]), not ${ }^{0} Z_{F, \Sigma}$. Fortunately, this causes no trouble in the sequel.

0.6. There is a notational inconsistency in [HZ1] (cf. (0.5)). Along with the $\bar{P}$ stratum we defined the $F$-stratum of $K_{f}^{1} \operatorname{Sh}(G, W \backslash X)_{\Sigma_{1}}$ in $[\mathrm{HZ1}, 1.7]$, where $F$ denotes the rational boundary component fixed by $\bar{P}$, also denoted $P_{F}$. However, in $\S 5.3$ of [HZ1] the $F$-stratum was denoted the $\bar{P}$-stratum. In the latter setting we had also defined $R$-strata for general parabolic subgroups $R$ of $G$ (i.e., not only for maximal parabolics) by the formula

$$
K_{f}^{1} \operatorname{Sh}(G, W \backslash X)_{\Sigma_{1}}^{R}=\bigcap_{\substack{P \supset R \\ P \text { maximal }}} K_{f}^{K_{1}^{1}} \operatorname{Sh}(G, W \backslash X)_{\Sigma_{1}}^{\bar{P}} .
$$


Here the final $\bar{P}$ should have been an $F$. However, we will retain the notation (0.6.1) and rename the $F$-stratum the $\bar{P}(*)$-stratum, denoted ${ }_{K_{f}^{1}} \operatorname{Sh}(G, W \backslash X)_{\Sigma_{1}}^{\bar{P}(*)}$. We recall that this is the union of the $G\left(\boldsymbol{A}_{f}\right)$-translates of the $P_{\alpha}$-strata for all maximal parabolics $P_{\alpha}$ conjugate to $\bar{P}$. Similarly, if $R$ is any standard parabolic subgroup of $G$, we let

$$
K_{f}^{1} \operatorname{Sh}(G, W \backslash X)_{\Sigma_{1}}^{R(*)}=\bigcap_{\substack{P \supset R \\ P \\ \text { maximal }}} K_{f}^{1} \operatorname{Sh}(G, W \backslash X)_{\Sigma_{1}}^{\bar{P}(*)} .
$$

In what follows, we will also use the less cumbersome notation of [HZ2, (1.5.2)] for closed boundary strata. Thus the Zariski closure of the $R$-stratum will be denoted $Z_{\Sigma}(R)$ and the Zariski closure of the $P(*)$-stratum will be denoted $Z_{\Sigma}(P(*))$, where appropriate.

0.7. Let $(G, X)$ be a (pure) Shimura datum. As in [HZ2] we let $\mathcal{P}(G)$ denote the set of rational $\mathbb{Q}$-parabolic subgroups of $G, \mathcal{P}_{\max }(G) \subset \mathcal{P}(G)$ the subset of maximal proper parabolics; the analogous notation is used for other reductive groups. To any $P \in \mathcal{P}_{\max }(G)$ we associate its Levi quotient $L_{P}$ and subgroups $G_{h, P}$ and $G_{\ell, P}$ of $L_{P}$, as in $[\mathrm{HZ} 1, \S 1.2]$, where the " $P$ " was sometimes dropped; see also (0.5). As in [HZ1], we choose liftings of $L_{P}$ to a subgroup of $P$, also denoted $L_{P}$. Sometimes we will restrict attention to standard parabolic subgroups, relative to a choice of minimal parabolic $P_{0}$ and then the Levi subgroups $L_{P}$ will all be assumed to contain $L_{P_{0}}$.

Assume $G^{\text {ad }}$ is $\mathbb{Q}$-simple. Then the set of standard maximal $\mathbb{Q}$-parabolics of $G$ $\mathcal{P}_{\max }(G)$ is totally ordered, as follows. Fix a connected component $X^{+} \subset X$. For each $P \in \mathcal{P}_{\max }(G)$ let $F(P)$ denote the rational boundary component of $X^{+}$fixed by the identity component $P(\mathbb{R})^{0}$ of $P(\mathbb{R})$. Then $P^{\prime} \leqslant P$ if and only if $F\left(P^{\prime}\right)$ is contained in the closure of $F(P)$.

As in $[\mathrm{HZ} 2,(2.2)]$, for $P \in \mathcal{P}_{\max }(G)$ we define an injection $\varepsilon_{P}: \mathcal{P}\left(G_{\ell, P}\right) \rightarrow \mathcal{P}(G)$, with image denoted $\mathcal{P}_{P}(G)$, such that

$$
\mathcal{P}(G)-\{G\}=\coprod_{P \in \mathcal{P}_{\max }(G)} \mathcal{P}_{P}(G) .
$$

If $R \in \mathcal{P}_{P}(G)$, then $R$ is said to be subordinate to $P$, and we write $\Pi(R)=P$; this is the case if $G_{h, P}$ is maximal among the $G_{h, Q}$ contained in the Levi quotient of $R$, as $Q$ runs through $\mathcal{P}_{\max }(G)$ (this was stated differently in [HZ2]). For brevity, we write $G_{h, R}=G_{h, P}$ if $R$ is subordinate to $P$. Let $R_{\ell, P}=R \cap G_{\ell, P}$ (in [HZ2,(2.2.3)], this was called $\left.\iota_{P}(R)\right)$. It is a $\mathbb{Q}$-parabolic subgroup of $G_{\ell, P}$, and every $\mathbb{Q}$-parabolic of $G_{\ell, P}$ is of the form $R_{\ell, P}$ for some $R$ subordinate to $P$. We also let $G_{\ell, R}$ denote the Levi subgroup $L_{R} \cap G_{\ell, P}$ of $R_{\ell, P}$.

0.8. A single object (sheaf, group, etc.) can be regarded as a complex with [at most] one non-zero term in degree 0 . A sheaf $\mathcal{S}$, or other such object, even if it "looks like it belongs in degree $q$ ", is placed in degree $q$ only by specifying the standard shift to the 
right: $\mathcal{S}[-q]$. In general, $\mathcal{S} \bullet[-q]$ has in degree $i$ what $\mathcal{S} \bullet$ has in degree $i-q$, whence the notation.

The cone $\mathcal{C} \bullet$ of a morphism of complexes $\phi: \mathcal{S}^{\bullet} \rightarrow \mathcal{T} \bullet$ has, by definition, as underlying sheaf $\mathcal{S} \bullet[1] \oplus \mathcal{T} \bullet$. Often it is more natural to work with $\mathcal{C} \bullet[-1]$; in that case, this shift will be specified. 


\section{CHAPTER 1}

\section{AUTOMORPHIC VECTOR BUNDLES ON MIXED SHIMURA VARIETIES}

In $\S 4$ of [HZ1], although we did not draw attention to this fact, we tacitly developed the basic theory of automorphic vector bundles on the mixed Shimura varieties, in the sense of Pink's thesis $[\mathrm{P}]$, that arise as boundary strata of toroidal compactifications of pure Shimura varieties. The present discussion recapitulates the constructions of $[\mathrm{HZ1}]$ in more orderly fashion and in the full generality of $[\mathrm{P}]$. The general setting includes the special case of Kuga's families of abelian varieties with additional structure, as well as their toroidal compactifications. These will not be discussed in the sequel but the results proved here appear to have interesting applications to the construction of mixed motives.

Most of the proofs are simple adaptations of the constructions in [H1, H2, H4], as well as [HZ1, HZ2,5.1]. Where this is the case, we keep the details to a minimum, referring to the analogous proofs in the earlier papers and thereby avoiding tedious repetition. We follow the strategy of [HZ1] by reducing purely geometric theorems about (mixed) Shimura varieties to local assertions, which we then prove on connected models of the form $\Gamma \backslash D$.

\subsection{Mixed Shimura varieties}

We refer to $[\mathrm{P}]$ for notation and precise definitions concerning mixed Shimura varieties. As in $[H Z 1,1.6]$, a mixed Shimura variety is defined by a pair $(Q, X)$, where $Q$ is a connected algebraic group over $\mathbb{Q}$ with a three step-filtration by normal subgroups

$$
\{1\} \subset W_{-2} Q \subset W_{-1} Q=R_{u} Q \subset W_{0} Q=Q
$$

and $\mathcal{X}$ is a homogeneous space for $Q(\mathbb{R}) \cdot W_{-2} Q(\mathbb{C})$ with a finite-to-one map

$$
h: X \longrightarrow \operatorname{Hom}\left(\underline{S}_{\mathbb{C}}, Q_{\mathbb{C}}\right) .
$$

We write $h_{x}$ for $h(x)$. It is assumed that $W_{-2} Q$ is commutative, and for any arithmetic subgroup $\Gamma \subset Q$ the quotient $T_{\Gamma}(Q, X)=\Gamma \cap W_{-2} Q(\mathbb{Q}) \backslash W_{-2} Q(\mathbb{C})$ is viewed as the set of complex points of the split torus with character group $\operatorname{Hom}\left(\Gamma \cap W_{-2} Q, \mathbb{Z}\right)$. The 
additional conditions satisfied by the pair $(Q, X)$ are listed in [P,Definition 2.1] and will be recalled as needed. The mixed Shimura variety is denoted $\operatorname{Sh}(Q, X)$.

We write $U_{Q}=W_{-2} Q, W_{Q}=W_{-1} Q, V_{Q}=W_{Q} / U_{Q}$, and drop the subscript $Q$ when it is understood, as it will be for most of the remainder of this section. Then $U$ and $V$ are commutative unipotent algebraic groups. In particular the exponential maps $\operatorname{Lie}(U) \rightarrow U$ and $\operatorname{Lie}(V) \rightarrow V$ are isomorphisms of vector groups. We let $G=Q / W$ be the maximal reductive quotient of $Q$.

Let

$$
\alpha_{1}: Q \longrightarrow Q / U, \quad \alpha_{2}: Q / U \longrightarrow G, \quad \alpha=\alpha_{2} \circ \alpha_{1}: Q \longrightarrow G
$$

be the natural maps. Conditions (i) and (ii) of [P, Definition 2.1] are that

(i) For any $x \in \mathcal{X}, \alpha_{1} \circ h_{x}$ is defined over $\mathbb{R}$;

(ii) For any $x \in \mathcal{X}, \alpha \circ h_{x} \circ w$ is a cocharacter of the center of $G$.

Pink's remaining conditions imply that the pair $\left(G,\left\{\alpha \circ h_{x} \mid x \in X\right\}\right)$ is a datum defining a pure Shimura variety. Let $\rho: Q \rightarrow G L(\Lambda)$ be a $\mathbb{Q}$-rational representation. Assume the weight morphism is defined over $\mathbb{Q}$ (see (1.1.7), below). Then for any $x \in \mathcal{X}$, the map $\rho \circ h_{x}$ defines a rational mixed Hodge structure on $\Lambda$ [P, Proposition 1.4], and, as $x$ varies, the family of $\rho \circ h_{x}$ defines a variation of mixed Hodge structures over $\mathcal{X}$. In particular, the family satisfies Griffiths transversality. Taking $\rho$ to be the adjoint representation, we obtain a family of mixed Hodge structures on $\operatorname{Lie}(Q)$. For $x \in \mathcal{X}$, let $Q_{x}^{0} \subset Q_{\mathbb{C}}$ denote the connected subgroup with Lie algebra $F_{x}^{0}(\operatorname{Lie}(Q))$; then $Q_{x}^{0}$ is the subgroup stabilizing the Hodge filtration $F_{\dot{x}}^{\bullet}(\Lambda)$ for any representation $(\rho, \Lambda)$. Let $\check{x}$ denote the quotient $Q_{\mathbb{C}} / Q_{x}^{0}$.

Let $\mathcal{H}_{\mathcal{X}}=\operatorname{Im}(h) \subset \operatorname{Hom}\left(\underline{S}_{\mathbb{C}}, Q_{\mathbb{C}}\right)$. The space $\mathcal{H}_{\mathcal{X}}$ has a $Q(\mathbb{R}) \cdot U(\mathbb{C})$-equivariant complex structure determined by the following property: Suppose $(\rho, \Lambda)$ is a faithful representation of $Q$. Let $\mathcal{F}(\rho)$ denote the variety of flags in $\Lambda$ containing the Hodge filtration $F_{y}$ attached to $\rho \circ y$ for any, and hence all, $y \in \mathcal{H}_{\mathcal{X}}$. Then the map

$$
\beta^{\prime}: \mathcal{H}_{\mathcal{X}} \longrightarrow \mathcal{F}(\rho) ; \quad y \longmapsto F_{y}
$$

is a complex analytic embedding [P,1.18(a)] that naturally factors through the homogeneous space $\check{X}(\mathbb{C})$. Since $h$ is a local diffeomorphism, this also determines a unique $Q(\mathbb{R}) \cdot U(\mathbb{C})$-equivariant complex structure on $\mathcal{H}$. The map $\beta=\beta^{\prime} \circ h: X \rightarrow \check{X}$ is called the Borel morphism.

The reflex field $E(Q, X)$ of the pair $(Q, X)$ is the field of definition of the conjugacy class of $h_{x} \circ \mu: \mathbb{G}_{m} \rightarrow Q$, with $\mu$ as in (0.1), for any $x \in X$ [P,Definition 11.1]. Just as in the case of pure Shimura varieties, the homogeneous algebraic variety $\check{x}$ is canonically a quotient of this conjugacy class, and for the same reasons has a natural rational structure over $E(Q, X)$ (cf. $[\mathrm{H} 1, \S 3])$.

Let $K_{f} \subset Q\left(\boldsymbol{A}_{f}\right)$ be a compact open subgroup. Then the mixed Shimura variety $K_{f} \operatorname{Sh}(Q, X)$ is defined as a complex analytic variety, just as in the pure case, by

$$
K_{f} \operatorname{Sh}(Q, X)(\mathbb{C})=Q(\mathbb{Q}) \backslash\left(X \times\left(Q\left(\boldsymbol{A}_{f}\right) / K_{f}\right)\right) .
$$


As $K_{f}$ varies, the $K_{f} \operatorname{Sh}(Q, X)(\mathbb{C})$ form a projective system, and one can define $\operatorname{Sh}(Q, X)(\mathbb{C})$ to be the inverse limit of the $K_{f} \operatorname{Sh}(Q, X)(\mathbb{C})$. Alternatively, we can view $\operatorname{Sh}(Q, X)(\mathbb{C})$ as shorthand for the projective system. In either case, we obtain an action of the group $Q\left(\boldsymbol{A}_{f}\right)$ on $\operatorname{Sh}(Q, X)(\mathbb{C})$ as "Hecke correspondences". However, we will mainly be concerned with the objects at finite level.

A morphism $(Q, X) \rightarrow\left(Q^{\prime}, X^{\prime}\right)$ of mixed Shimura data is a pair consisting of a group homomorphism $Q \rightarrow Q^{\prime}$, defined over $\mathbb{Q}$, and a holomorphic map $X \rightarrow X^{\prime}$, compatible with the group actions on the two sides. To a morphism $\phi:(Q, X) \rightarrow\left(Q^{\prime}, X^{\prime}\right)$ of mixed Shimura data we can associate holomorphic maps of mixed Shimura varieties: if $K_{f} \subset Q\left(\boldsymbol{A}_{f}\right)$ and $K_{f}^{\prime} \subset Q^{\prime}\left(\boldsymbol{A}_{f}\right)$ are such that $\phi\left(K_{f}\right) \subset K_{f}^{\prime}$, then there is a natural map

$$
[\phi]:{ }_{K_{f}} \operatorname{Sh}(Q, X)(\mathbb{C}) \longrightarrow{ }_{K_{f}^{\prime}} \operatorname{Sh}\left(Q^{\prime}, X^{\prime}\right)(\mathbb{C})
$$

$([\mathrm{P}, 3.4])$. As in the pure case, to a morphism of mixed Shimura data we obtain a map of reflex fields in the opposite direction: $E\left(Q^{\prime}, X^{\prime}\right) \subset E(Q, X)$.

In particular, we can take $Q^{\prime}=Q / U$ or $Q^{\prime}=G=Q / W$ in the above construction, and $Q \rightarrow Q^{\prime}$ to be the natural projection. If we define $X^{\prime}$ to be the quotient of $\mathcal{X}$ by $U(\mathbb{C})$, resp. by $U(\mathbb{C}) \cdot W(\mathbb{R})$, we thus obtain morphisms of Shimura data $(Q, X) \rightarrow$ $\left(Q^{\prime}, X^{\prime}\right)$. Writing $U \backslash X$ in place of $U(\mathbb{C}) \backslash X$ and $W \backslash X$ in place of $U(\mathbb{C}) \cdot W(\mathbb{R}) \backslash X$, we thus obtain a short sequence of morphisms of mixed Shimura varieties:

$$
\operatorname{Sh}(Q, X)(\mathbb{C}) \stackrel{\pi_{2}}{\longrightarrow} \operatorname{Sh}(Q / U, U \backslash X)(\mathbb{C}) \stackrel{\pi_{1}}{\longrightarrow} \operatorname{Sh}(G, W \backslash X)(\mathbb{C}) .
$$

We have already noted that $(G, W \backslash X)$ is a pure Shimura datum.

Among the main theorems of $[\mathrm{P}]$ are the existence of natural algebraic structures on the mixed Shimura varieties $\operatorname{Sh}(Q, X)(\mathbb{C})$, the existence of smooth projective toroidal compactifications of the mixed Shimura varieties $K_{f} \operatorname{Sh}(Q, X)(\mathbb{C})$ (provided the level subgroup $K_{f}$ is neat, in the sense of $[\mathrm{P}, 0.6]$ ), and the existence of canonical models of $\operatorname{Sh}(Q, X)(\mathbb{C})$ and its toroidal compactifications over the reflex field $E(G, X)$ $[\mathrm{P}$, Theorems 9.21, 11.18, and 12.4].(1) We mention for the sake of completeness that any $K_{f}$ contains a neat subgroup of finite index, and that if $K_{f}$ is neat then any subgroup of finite index in $K_{f}$ is also neat. The algebraic structures are "natural" in that they are compatible with the morphisms introduced above, including the Hecke correspondences $Q\left(\boldsymbol{A}_{f}\right)$ and the morphisms [ $\left.\phi\right]$ of (1.1.4) associated to morphisms $\phi:(Q, X) \rightarrow\left(Q^{\prime}, X^{\prime}\right)$ of mixed Shimura data. The algebraization of the mixed Shimura varieties is actually accomplished by constructing ample line bundles on certain toroidal compactifications.

The canonical model, denoted $\operatorname{Sh}(Q, X)$, is defined, as in the pure case, by functoriality with respect to morphisms of mixed Shimura data $(T, Y) \rightarrow(Q, X)$. Here $T$

(1) Of course, Pink's theorems presuppose the existence of canonical models in the pure case; see [M2] for references, as well as the recent articles of Moonen and Milne [Mo,M3], filling an apparent oversight in the literature. 
is a torus, the "symmetric space" $Y$ is a finite set of points, and the canonical model of $\operatorname{Sh}(T, Y)$ is defined by the analogue of the Shimura-Taniyama reciprocity law for complex multiplication. The points contained in the image of a morphism of the form $\operatorname{Sh}(T, Y) \rightarrow \operatorname{Sh}(Q, X)$, or the corresponding points at finite level, are called special points. Thus the canonical models constructed by Pink, like those of Shimura, are characterized by the reciprocity law at the special points. Details can be found in $[\mathrm{P}, 11.5]$.

1.1.6. The (partial) toroidal compactifications of ${ }_{K_{f}} \operatorname{Sh}(Q, \mathcal{X})(\mathbb{C})$ are associated to combinatorial data, just as in the pure case [cf. HZ1, 1.4, 1.7]. Following the conventions introduced in [HZ1], the set of combinatorial data will be denoted $\Sigma$ and will be called a $K_{f}$-admissible family of fans for $(Q, X)$ (Pink denotes the set of data $\mathcal{S}$ and calls it a $K_{f}$-admissible partial cone decomposition; see [P, 6.4]). This aspect of Pink's theory was discussed in [HZ1, 1.6 and 4.1] in connection with the mixed Shimura varieties that arise as boundary strata of pure Shimura varieties, and the general case is identical. The (partial) toroidal compactification of $K_{f} \operatorname{Sh}(Q, X)(\mathbb{C})$ associated to the $K_{f}$-admissible family of fans $\Sigma$ is denoted $K_{f} \operatorname{Sh}(Q, \mathcal{X})_{\Sigma}(\mathbb{C})$. We assume $\Sigma$ satisfies the hypotheses of $\left[\mathrm{P}\right.$, Theorem 12.4]. Then by [loc. cit.], $K_{f} \operatorname{Sh}(Q, \mathcal{X})_{\Sigma}(\mathbb{C})$ exists as a complex algebraic variety, and descends to a scheme $K_{f} \operatorname{Sh}(Q, \mathcal{X})_{\Sigma}$ over the reflex field $E(Q, X)$. Under supplementary hypotheses on $\Sigma$, we may assume $K_{f} \operatorname{Sh}(Q, X)_{\Sigma}$ to be quasi-projective; in this case we call $\Sigma$ quasi-projective. Just as in the pure case, the set of all $K_{f}$-admissible families of fans is an inverse system under the relation of refinement. If $\Sigma_{1}$ is a refinement of $\Sigma_{2}$ then there is a natural $\operatorname{morphism}_{K_{f}} \operatorname{Sh}(Q, X)_{\Sigma_{1}} \rightarrow_{K_{f}} \operatorname{Sh}(Q, X)_{\Sigma_{2}}[\mathrm{P}, 6.7(\mathrm{~b})]$; moreover this morphism is projective [KKMS], and the set of quasi-projective $\Sigma$ is cofinal in the set of all families of fans [P, Theorem 9.21].

If $K_{f}$ is neat, then we may also choose $\Sigma$ such that $K_{f} \operatorname{Sh}(Q, \mathcal{X})_{\Sigma}$ is smooth and the complement $\partial_{K_{f}} \operatorname{Sh}(Q, X)_{\Sigma}=K_{f} \operatorname{Sh}(Q, X)_{\Sigma}-K_{f} \operatorname{Sh}(Q, X)$ is a divisor with normal crossings. In this case, $K_{f} \operatorname{Sh}(Q, \mathcal{X})_{\Sigma}$ is said to be $\mathrm{SNC}$.

1.1.7. On the morphism $h$ of (1.1.2). - Say a pure Shimura datum $\left(G_{1}, X_{1}\right)$ is standard if the associated map $h: X_{1} \rightarrow \operatorname{Hom}\left(\underline{S}_{\mathbb{C}}, Q_{\mathbb{C}}\right)$ of (1.1.2) is one-to-one. It follows from Proposition 2.11 of $[\mathrm{P}]$ that there is a standard Shimura datum $\left(G_{1}, X_{1}\right)=(G, h(X))$ and a Shimura datum $(T, \mathcal{Y})$, with $T$ a torus, such that $(G, X)$ admits an embedding

$$
(G, X) \longleftrightarrow\left(G_{1}, X_{1}\right) \times(T, \mathcal{Y})
$$

In other words the map $h$ associated to $(G, X)$ is concentrated on the second (toric) factor. 
Let $(G, W \backslash X)$ denote the pure Shimura datum attached to the mixed Shimura datum $(Q, X)$. Consider the following hypotheses:

For any $h \in W \backslash X$, the weight morphism $h \circ w: \mathbb{G}_{m, \mathbb{R}} \rightarrow G_{\mathbb{R}}$ is defined over $\mathbb{Q}$.

Let $\bar{Z}_{G}$ denote the maximal $\mathbb{R}$-split torus of the center of $G$ that is $\mathbb{Q}$-anisotropic. Then $\bar{Z}_{G}$ is trivial.

The Shimura datum $(Q, X)$ will be called motivic if (1.1.7.2) holds. We assume this to be the case. Taking $(G, X)$ in (1.1.7.1) to be $(G, W \backslash X)$, it is then easy to see that we may assume $\left(G_{1}, X_{1}\right)$ and $(T, \mathcal{Y})$ to be motivic as well. If $G$ also satisfies (1.1.7.3), then we may assume this to be the case for $G_{1}$ and $T$ as well.

We wish to determine the failure of $(T, \mathcal{Y})$ to be standard. Let $h: \mathcal{Y} \rightarrow \mathcal{H}_{\mathcal{Y}}$ with $\mathcal{H}$ as in (1.1.3). First note that there is a unique Shimura datum $\left(\mathbb{G}_{m}, \mathcal{Y}( \pm)\right)$ such that $\mathcal{H}_{\mathcal{Y}( \pm)}$ is the character $z \mapsto z \bar{z}$ of $\underline{S}$, and such that $\mathcal{Y}( \pm)$ has two connected components. For any integer $M$, the character $t \rightarrow t^{M}$ is a morphism $\phi_{M}: \mathbb{G}_{m} \rightarrow \mathbb{G}_{m}$, and there is a mixed Shimura datum $\left(\mathbb{G}_{m}, \mathcal{Y}(M, \pm)\right)$ such that $\phi_{M}$ defines a morphism

$$
\left(\mathbb{G}_{m}, \mathcal{Y}( \pm)\right) \longrightarrow\left(\mathbb{G}_{m}, \mathcal{Y}(M, \pm)\right)
$$

of Shimura data. (Actually, $\phi_{M}$ defines two morphisms. $\mathcal{Y}(M, \pm$ ) has two connected components, interchanged by complex conjugation on cyclotomic fields. Composing one choice of $\phi_{M}$ with exchange of the components of $\mathcal{Y}(M, \pm)$ defines a second morphism of the form (1.1.7.4).) We let $\mathcal{Y}(M)$ denote the single character $z \mapsto(z \bar{z})^{M}$. Then every $\left(\mathbb{G}_{m}, \mathcal{Y}\right)$ is of the form $\left(\mathbb{G}_{m}, \mathcal{Y}(M)\right)$ or $\left(\mathbb{G}_{m}, \mathcal{Y}(M, \pm)\right)$ for some $M$.

Suppose $T$ satisfies (1.1.7.3). Let $T_{0}$ denote the maximal $\mathbb{Q}$-anisotropic subtorus of $T, T_{1}=T / T_{0}$. There is a Shimura datum $\left(T_{1}, \mathcal{Y}_{1}\right)$ and a morphism $(T, \mathcal{Y}) \rightarrow\left(T_{1}, \mathcal{Y}_{1}\right)$ whose first factor is the canonical map; the datum $\left(T_{1}, \mathcal{Y}_{1}\right)$ is the quotient of $(T, \mathcal{Y})$ by $T_{0}$, in the sense of $\left[\mathrm{P}\right.$, Proposition 2.9]. By (1.1.7.3), the map $\pi_{0}(T(\mathbb{R})) \rightarrow \pi_{0}\left(T_{1}(\mathbb{R})\right)$ is injective; it then follows from the proof of [P, loc. cit.] that the map $\mathcal{Y} \rightarrow \mathcal{Y}_{1}$ is also injective. On the other hand, $T_{1} \stackrel{\sim}{\longrightarrow} \mathbb{G}_{m}^{r}$ for some non-negative integer $r$. Thus there is an embedding of Shimura data

$$
(T, \mathcal{Y}) \longrightarrow\left(T, \mathcal{H}_{\mathcal{Y}}\right) \times\left(\mathbb{G}_{m}^{r}, \mathcal{Y}_{1}\right)
$$

By incorporating $\left(T, \mathcal{H}_{\mathcal{Y}}\right)$ into the first factor on the right of (1.1.7.1), we see that, assuming $G$ satisfies (1.1.7.3), there is an embedding of pure Shimura data

$$
(G, X) \longleftrightarrow\left(G_{1}, X_{1}\right) \times(T, \mathcal{Y})
$$

where $\left(G_{1}, X_{1}\right)$ is standard and $T$ is a $\mathbb{Q}$-split torus.

Via (1.1.7.6), the general theory of pure Shimura data, in Pink's sense, is reduced to the theory of standard Shimura data and the additional cases $\left(\mathbb{G}_{m}, \mathcal{Y}(M, \pm)\right)$. We use this reduction in explaining the construction of automorphic vector bundles in the following section. 
1.1.8. We will carry out certain calculations on connected components of the mixed Shimura varieties. Thus if $\Gamma \subset Q(\mathbb{Q})$ is a congruence subgroup, and if $X^{0} \subset X$ is a connected component, we let $M_{\Gamma}=M_{\Gamma}\left(X^{0}\right)=\Gamma \backslash \mathcal{X}^{0}$. The connected components of the mixed Shimura variety $K_{f} \operatorname{Sh}(Q, X)(\mathbb{C})$ are all of the form $M_{\Gamma}\left(\mathcal{X}^{0}\right)[\mathrm{P}, 3.2]$. The notation $\left(X^{0}\right)$ will generally be dropped, since our assertions are independent of the choice of a connected component.

Similarly, a toroidal compactification of $M_{\Gamma}$ will be denoted $M_{\Gamma, \Sigma}$. Here it is understood that $\Sigma$ is a $\Gamma$-admissible family of fans for the pair $\left(Q, X^{0}\right)$, with the obvious definition. Alternatively, if $M_{\Gamma}$ is realized as a connected component of some $K_{f} \operatorname{Sh}(Q, X)(\mathbb{C})$, then we may take $\Sigma$ to be a $K_{f}$-admissible family of fans for $(Q, X)$, and define $M_{\Gamma, \Sigma}$ to be the closure of $M_{\Gamma}$ in $K_{f} \operatorname{Sh}(Q, \mathcal{X})_{\Sigma}(\mathbb{C})$. Again, this distinction will be irrelevant in calculations involving connected components.

1.1.9. Boundary strata. - For future reference, we introduce notation for the stratification of the toroidal boundary. For this we need to recall the classification of rational boundary components of $\operatorname{Sh}(Q, X)$. Let $G^{\text {ad }}=G_{1} \times \cdots \times G_{r}$ be the decomposition into $\mathbb{Q}$-simple factors. Let $P_{i} \subset G_{i}$ be a $\mathbb{Q}$-parabolic subgroup for every $i$, and let $P \subset Q$ be the inverse image of $P_{1} \times \cdots \times P_{r}$. Then [P, Definition 4.5] $P$ is an admissible $\mathbb{Q}$-parabolic subgroup of $Q$ if every $P_{i}$ is either equal to $G_{i}$ or to a maximal proper $\mathbb{Q}$-parabolic subgroup of $G_{i}$. As in the pure case (see $(0.7)$ ), we let $\mathcal{P}(Q)$ denote the set of admissible $\mathbb{Q}$-parabolics of $Q$.

In particular, every admissible $\mathbb{Q}$-parabolic subgroup of $Q$ is the pullback of an admissible $\mathbb{Q}$-parabolic subgroup of $G$. In what follows we refer to (0.6) for notation concerning the boundary strata in the pure case. Let $\bar{P}$ be an admissible $\mathbb{Q}$-parabolic subgroup of $G, P$ the corresponding admissible parabolic of $Q$. We define the $\bar{P}$ ${\text { stratum } K_{f}^{1}} \operatorname{Sh}(G, W \backslash X)_{\Sigma_{1}}^{\bar{P}}$ of $K_{f}^{1} \operatorname{Sh}(G, W \backslash X)_{\Sigma_{1}}$ as in [HZ1,1.7], and define the $P$ stratum of $K_{f} \operatorname{Sh}(Q, \mathcal{X})_{\Sigma}$ to be

$$
K_{f} \operatorname{Sh}(Q, X)_{\Sigma}^{P}=\left(\pi_{2, \Sigma} \circ \pi_{1, \Sigma}\right)^{-1}\left({ }_{K_{f}^{1}} \operatorname{Sh}(G, W \backslash X)_{\Sigma_{1}}^{\bar{P}}\right) .
$$

We note that these strata are in general not Zariski closed.

If now $P$ is any $\mathbb{Q}$-parabolic subgroup of $Q$, we define the $P$-stratum of $K_{f} \operatorname{Sh}(Q, X)_{\Sigma}$ by analogy with (1.1.10); the $P(*)$-stratum ${ }_{K_{f}} \operatorname{Sh}(Q, X)_{\Sigma}^{P(*)}$ of ${ }_{K_{f}} \operatorname{Sh}(Q, X)_{\Sigma}$ is defined in the same way, with $\bar{P}$ replaced by $\bar{P}(*)$.

\subsection{Construction of automorphic vector bundles}

We keep the notation of $\S 1.1$. Henceforward, we make the following standard

Hypothesis 1.2.1. - The Shimura datum $(Q, X)$ is motivic (1.1.7.2) and satisfies condition (1.1.7.3). 
Hypothesis 1.2.1 guarantees that any algebraic representation of $G$ gives rise to a rational local system over $\operatorname{Sh}(Q, X)(\mathbb{C})$, in the sense of $[\mathrm{H} 1,3.14]$. The hypothesis also allows us to define the standard principal bundle $I(Q, X)(\mathbb{C})$ over $\operatorname{Sh}(Q, \mathcal{X})(\mathbb{C})$ as the complex analytic variety

$$
I(Q, X)(\mathbb{C})=\lim _{\longleftarrow} Q(\mathbb{Q}) \backslash\left(Q(\mathbb{C}) \times X \times\left(Q\left(\boldsymbol{A}_{f}\right) / K_{f}\right)\right) .
$$

Here as in $(1.1 .3)$, the group $Q(\mathbb{Q})$ acts diagonally on the left on the product of the three factors. That this is indeed a principal bundle follows in the usual way from Hypothesis 1.2.1 (cf. [M2, Proposition 3.3]). Again, this can be viewed as an inverse system of principal $Q(\mathbb{C})$-bundles

$$
K_{f} I(Q, X)(\mathbb{C}) \longrightarrow K_{f} \operatorname{Sh}(Q, X)(\mathbb{C}) .
$$

REMARK. - One obtains slightly more generality by dropping hypothesis (1.2.1) and restricting attention to algebraic representations of $G$ trivial on the subgroup $\bar{Z}(G)$ defined in (1.1.7.3). We leave it to the interested reader to carry out the necessary modifications.

The natural projection $I(Q, X)(\mathbb{C}) \rightarrow \operatorname{Sh}(Q, X)(\mathbb{C})$ is denoted $q=q_{Q}, x$. On the other hand, there is a natural map $p=p_{Q, x}: I(Q, \mathcal{X})(\mathbb{C}) \rightarrow \check{X}(\mathbb{C})$ defined in terms of (1.2.2) by

$$
p\left(q, x, q_{f}\right)=q^{-1} \cdot \beta(x), q \in Q(\mathbb{C}), x \in \mathcal{X}, q_{f} \in\left(Q\left(\boldsymbol{A}_{f}\right) / K_{f}\right) .
$$

The right-hand side is obviously invariant under left-translation by $Q(\mathbb{Q})$ and defines a holomorphic map.

Proposition 1.2.4. - The standard principal bundle $I(Q, X)(\mathbb{C})$ can be given a natural structure of algebraic principal bundle $I(Q, X)_{\mathbb{C}}$ over $\operatorname{Sh}(Q, X)_{\mathbb{C}}$. Moreover, $I(Q, X)_{\mathbb{C}}$ has a canonical $Q\left(\boldsymbol{A}_{f}\right)$-equivariant model $I(Q, X)$ over the reflex field $E(Q, X)$, and the morphisms $p_{Q, X}$ and $q_{Q, X}$ descend to yield a canonical diagram

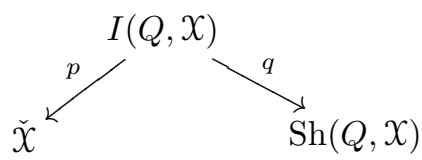

over $E(Q, X)$

Explanation-Definition 1.2.5. - The canonical model to which we refer in the proposition is defined by combining the definitions of Pink (for canonical models of mixed Shimura varieties) and Milne (for canonical models of standard principal bundles over pure Shimura varieties, see [M1]). Unfortunately, a complete explanation requires additional notation. We take as given the existence of a canonical model on $I(T, \mathcal{Y})$ when $T$ is a torus. When $\mathcal{Y}$ is a point, this canonical model is defined as in [M1] in terms of the period torsor. The discussion in (1.1.7) reduces the general case to the case where $T=\mathbb{G}_{m}$, which will be treated in the appendix (1.2.A), below. 
If $\left(Q^{\prime}, X^{\prime}\right) \rightarrow(Q, X)$ is an embedding of mixed Shimura data then $E(Q, X) \subset$ $E\left(Q^{\prime}, X^{\prime}\right)[\mathrm{P}, 11.2(\mathrm{~b})]$. Then a canonical model on $I(Q, X)$ is a $Q\left(\boldsymbol{A}_{f}\right)$-equivariant model such that, for any embedding of mixed Shimura data $(T, \mathcal{Y}) \rightarrow(Q, \mathcal{X})$, with $T$ a torus, the natural morphism $I(T, \mathcal{Y}) \rightarrow I(Q, \mathcal{X})$ descends to $E(T, \mathcal{Y})$. One verifies as for pure Shimura varieties that the canonical model is unique, if it exists.

Proof of Proposition 1.2.4. - For any positive integer $g$, we let $\left(P_{2 g}, X_{2 g}\right)$ be the mixed Shimura datum defined in $[\mathrm{P}, 2.25]$. It is a mixed Shimura datum associated to the genus $g$ (maximal) boundary component of the Siegel modular Shimura variety of genus $g+1$, denoted $\operatorname{Sh}\left(G S p(g+1), \mathfrak{S}_{g+1}^{ \pm}\right)$. Here $G S p(g+1)$ is the group of similitudes of a $2(g+1)$-dimensional symplectic space and $\mathfrak{S}_{g+1}^{ \pm}$is the union of the Siegel upper and lower half-spaces of genus $g+1$. We can realize the parabolic subgroup $P_{2 g} \subset G S p(g+1)$ as the stabilizer of a rational genus $g$ boundary component of $\mathfrak{S}_{g+1}^{ \pm}$. For a cofinal subset of the set of level subgroups $K_{f} \subset P_{2 g}\left(\boldsymbol{A}_{f}\right)$, the associated mixed Shimura variety $K_{f} \operatorname{Sh}\left(P_{2 g}, X_{2 g}\right)$ can thus be realized as a union of boundary strata of a toroidal compactification of $\operatorname{Sh}\left(G S p(g+1), \mathfrak{S}_{g+1}^{ \pm}\right)$(at an appropriate finite level, depending on $K_{f}$ ). Since the center of the unipotent radical of $P_{2 g}$ is one-dimensional, the realization as a (union of) boundary strata does not depend on the choice of toroidal compactifications.

Now when $(Q, X)$ is a pure Shimura datum and the natural map $X \rightarrow \mathcal{H}$ is the identity - i.e., $(Q, X)$ is a standard Shimura datum - then the proposition is proved in [H1] and [M1]. When $Q$ is a torus, the proposition is tautologically true, whether or not $X$ is connected. On the other hand, when $K_{f} \operatorname{Sh}(Q, X)$ is a (union of) boundary strata of a toroidal compactification attached to a rational boundary component of a pure Shimura variety, then the canonical model of $I(Q, X)_{\mathbb{C}}$ is constructed in [HZ1, Lemma 4.5.8]. The fact that this model is canonical is an immediate consequence of [HZ1, Lemma 4.6.9], since, when $(T, \mathcal{Y})$ is as in 1.2 .5 , the morphism $\operatorname{Sh}(T, \mathcal{Y}) \rightarrow \operatorname{Sh}(Q, X)$ factors through some section of the natural map $\pi_{1} \circ \pi_{2}: \operatorname{Sh}(Q, \mathcal{X}) \rightarrow \operatorname{Sh}(G, W \backslash X)$.

We remark that the $Q\left(\boldsymbol{A}_{f}\right)$-equivariance is not stated explicitly in [HZ1], and indeed the statement needs to be modified for boundary strata of toroidal compactifications to take account of the combinatorial data defining the compactification. We will only apply [HZ1, Lemma 4.5.8] to the case of $\left(P_{2 g}, X_{2 g}\right)$. The fan in this case is uniquely determined. Therefore, the $P_{2 g}\left(\boldsymbol{A}_{f}\right)$-equivariance follows immediately from the $G S p(g+1)\left(\boldsymbol{A}_{f}\right)$-equivariance of the canonical model of the standard principal bundle over $\operatorname{Sh}\left(G S p(g+1), \mathfrak{S}_{g+1}^{ \pm}\right)$.

It follows in the usual way that if $\left(Q^{\prime}, X^{\prime}\right) \hookrightarrow(Q, X)$ is an embedding of Shimura data, then the existence of a canonical model for $I(Q, X)$ implies the existence of a canonical model for $I\left(Q^{\prime}, X^{\prime}\right)$. It thus follows from what has been established up to 
this point that, if $(Q, \mathcal{X})$ admits an embedding of the form

$$
(Q, \mathcal{X}) \longrightarrow\left(G_{1}, X_{1}\right) \times(T, \mathcal{Y}) \times \prod_{i=1}^{n}\left(P_{2 g}, X_{2 g}\right)
$$

where $\left(G_{1}, X_{1}\right)$ is a standard Shimura datum, $T$ is a $\mathbb{Q}$-split torus, and $n$ is a nonnegative integer, then the proposition holds for $(Q, \mathcal{X})$.

Lemma 2.26 of $[\mathrm{P}]$ asserts that, we can replace $(Q, \mathcal{X})$ by a new pair $\left(Q^{\prime}, \mathcal{X}^{\prime}\right)$, where $Q^{\prime}$ is an extension of $Q$ by $U_{0} \simeq \mathbb{G}_{a}$ (contained in the center of the unipotent radical of $\left.Q^{\prime}\right)$ of a specific type, called a $\left(P_{0}, X_{0}\right) \rightarrow\left(\mathbb{G}_{m, \mathbb{Q}}, \mathcal{H}_{0}\right)$-torsor, such that $\left(Q^{\prime}, X^{\prime}\right)$ admits an embedding of the form (1.2.6); the fact that we can take $T \mathbb{Q}$-split is (1.1.7.6). Thus we have the $Q^{\prime}$-torsor $I\left(Q^{\prime}, X^{\prime}\right)$ over $\operatorname{Sh}\left(Q^{\prime}, X^{\prime}\right)$ with its canonical model over $E\left(Q^{\prime}, X^{\prime}\right)$, which is easily checked to equal $E(Q, X)$. The quotient of $I\left(Q^{\prime}, X^{\prime}\right)$ by $U_{0}$ is then a $Q$-torsor over $\operatorname{Sh}\left(Q^{\prime}, \mathcal{X}^{\prime}\right)$, which is in turn a $\mathbb{G}_{m}$-torsor over $\operatorname{Sh}(Q, \mathcal{X})$. Over $\mathbb{C}, U_{0} \backslash I\left(Q^{\prime}, X^{\prime}\right)$ is obviously isomorphic to the pullback of $I(Q, \mathcal{X})_{\mathbb{C}}$ from $\operatorname{Sh}(Q, \mathcal{X})$. Thus the $\mathbb{G}_{m}$-action on $\operatorname{Sh}\left(Q^{\prime}, X^{\prime}\right)$ lifts canonically to an action on $U_{0} \backslash I\left(Q^{\prime}, X^{\prime}\right)$ and thus defines an $E(Q, X)$-rational model $I(Q, \mathcal{X})$ of $I(Q, X)_{\mathbb{C}}$. Since any morphism $(T, \mathcal{Y}) \rightarrow(Q, \mathcal{X})$, with $T$ a torus, lifts to a morphism $(T, \mathcal{Y}) \rightarrow\left(Q^{\prime}, X^{\prime}\right)$, the canonicity of $I(Q, X)$ follows from that of $I\left(Q^{\prime}, X^{\prime}\right)$.

It remains to show that the morphism $p: I(Q, \mathcal{X}) \rightarrow \check{X}$ can be descended to $E(Q, X)$. This follows again by reduction to the individual factors on the right-hand side of (1.2.6). For the first factor this is in [H1] and [M1], for the second factor it's trivial, and for the third factor it is contained in Lemma 4.5.8 of [HZ1]. This completes the proof.

Given the diagram in Proposition 1.2.4, automorphic vector bundles can be constructed on $\operatorname{Sh}(Q, X)$ by the procedure described in [H1] and [M1], which we now recall. Let $\mathcal{W}$ be a $Q$-homogeneous vector bundle on $\check{X}$. Then $p^{*} \mathcal{W}$ is a $Q$-equivariant vector bundle on $I(Q, \mathcal{X})$, hence descends to a $Q\left(\boldsymbol{A}_{f}\right)$-equivariant vector bundle $[\mathcal{W}]$ on $\operatorname{Sh}(Q, X)$. The functor $\mathcal{W} \mapsto[\mathcal{W}]$ is rational over $E(Q, X)$, by construction, and defines canonical models, just as in $[\mathrm{M} 1]$, on the complex points of $[\mathcal{W}]$, which just as in the classical case can be identified with a locally homogeneous vector bundle:

$$
[\mathcal{W}](\mathbb{C}) \stackrel{\sim}{\longrightarrow}\left(Q(\mathbb{Q}) \backslash \beta^{*}(\mathcal{W}) \times\left(Q\left(\boldsymbol{A}_{f}\right) / K_{f}\right)\right) .
$$

A $Q$-homogeneous vector bundle on $\check{X}$ is called locally-finite if it is the direct limit of finite-rank homogeneous vector bundles on $\check{X}$. The functor above obviously is defined on locally-finite homogeneous vector bundles and satisfies $\left[\lim _{\longrightarrow} \mathcal{W}_{\alpha}\right]=\underline{\lim }\left[\mathcal{W}_{\alpha}\right]$. Defining $[\mathcal{W}](\mathbb{C})$ by $(1.2 .7)$, we resume this discussion as follows:

Proposition 1.2.8. - The functor $\mathcal{W} \mapsto[\mathcal{W}](\mathbb{C})$, defined by $(1.2 .7)$, maps the tensor category of locally finite $Q$-homogeneous algebraic vector bundles on $\check{X}$ to the tensor category of $Q\left(\boldsymbol{A}_{f}\right)$-equivariant algebraic vector bundles on $\operatorname{Sh}(Q, X)$. This functor is rational over $E(Q, X)$ and is functorial with respect to morphisms $\left(Q^{\prime}, X^{\prime}\right) \rightarrow(Q, X)$ 
of mixed Shimura data. When $(Q, X)$ is a pure Shimura datum, the functor coincides with the one defined in [H1, M1]. In particular, the bundles $[\mathcal{W}]$ have canonical models in the sense of [M1].

The final assertion in (1.2.8) is an immediate consequence of the others, since the canonical models are defined with respect to embeddings of pure Shimura data of the form $(T, \mathcal{Y})$, with $T$ a torus. Bundles of the form $[\mathcal{W}]$ are called automorphic vector bundles. The automorphic vector bundles form a tensor subcategory of the category of all $Q\left(\boldsymbol{A}_{f}\right)$-equivariant vector bundles on $\operatorname{Sh}(Q, X)$.

Let $(\rho, L)$ be an algebraic representation of $Q$. We say $(\rho, L)$ is locally finite if it is the direct limit of finite-dimensional (algebraic) representations of $Q$. An automorphic vector bundle $[\mathcal{W}]$ on $\mathrm{Sh}$ is called flat if $\mathcal{W}$ is isomorphic to a $Q$-homogeneous vector bundle on $\check{X}$ of the form $L \otimes \mathcal{O}_{\check{X}}$, where $(\rho, L)$ is a locally-finite representation of $Q$. We then write $[\mathcal{W}]=\widetilde{L}$. Equivalently, on any connected component $M_{\Gamma} \subset \operatorname{Sh}(\mathbb{C})$, $[\mathcal{W}]$ is the direct limit of vector bundles associated to finite-dimensional algebraic representations of the fundamental group $\Gamma$ of $M_{\Gamma}$ (here "algebraic representation of $\Gamma$ " is shorthand for "restriction to $\Gamma$ of an algebraic representation of $Q$ ".) A flat vector bundle always has a canonical flat connection $\nabla=\nabla_{L}: \widetilde{L} \rightarrow \widetilde{L} \otimes \Omega_{\mathrm{Sh}}^{1}$, induced by functoriality from the constant connection $1 \otimes d: L \otimes \mathcal{O}_{\check{x}} \rightarrow L \otimes \Omega_{\check{x}}^{1}$.

The standard principal bundle $I(Q, X)$ has the following functorial description. Let $(\rho, L)$ be a faithful $\mathbb{Q}$-rational representation with contragredient $\left(\rho^{*}, L^{*}\right)$. Let $s_{i} \in L^{\otimes n_{i}} \otimes\left(L^{*}\right)^{\otimes m_{i}}(i=1, \ldots, r)$ be a finite set of $\mathbb{Q}$-rational tensors such that $Q$ is the subgroup of $G L(L)$ stabilizing all $s_{i}$. Let $\mathbf{1}$ denote $\mathbb{Q}$, viewed as a trivial representation of $\mathrm{Q}$. For each $i$, the morphism of $Q$-representations

$$
\alpha_{i}: \mathbf{1} \longrightarrow L^{\otimes n_{i}} \otimes\left(L^{*}\right)^{\otimes m_{i}} ; \quad \alpha_{i}(1)=s_{i}
$$

defines by functoriality an injective homomorphism

$$
\widetilde{\alpha}_{i}: \mathcal{O}_{\mathrm{Sh}} \longrightarrow \widetilde{L}^{\otimes n_{i}} \otimes\left(\widetilde{L}^{*}\right)^{\otimes m_{i}}
$$

of automorphic vector bundles. Let $\widetilde{s_{i}}=\widetilde{\alpha}_{i}(1), i=1, \ldots, r$. It is then tautological that $I(Q, \mathcal{X})$ represents the functor that, to any Sh-scheme $T$, associates

$$
\left\{f: \widetilde{L}_{T} \stackrel{\sim}{\longrightarrow} L_{T} \mid\left(f^{\otimes n_{i}} \otimes\left(f^{*}\right)^{\otimes m_{i}}\right)\left(\widetilde{s_{i}}\right)=s_{i} \otimes 1\right\} .
$$

Here $L_{T}$ is the constant bundle $L \otimes \mathcal{O}_{T}$ and the remaining notation is obvious. In fact, the construction of the canonical model of $I(Q, \mathcal{X})$ in $[\mathrm{H} 1, \mathrm{M} 1]$ in the pure case proceeds by constructing the flat filtered automorphic vector bundle $\widetilde{L}$ and showing that it has an appropriate kind of canonical model; in particular, that the $\left[s_{i}\right]$ are rational over $E(Q, \mathcal{X})$. Then $I(Q, \mathcal{X})$ is defined by $(1.2 .10)$, and is shown as in [H1, Remark 4.9(1); M2, Remark 3.3] to be independent of the choice of $(\rho, L)$.

The morphism $\pi_{2}: \operatorname{Sh}(Q, X) \rightarrow \operatorname{Sh}(Q / U, U \backslash X)$ is a locally constant pro-torus fibration over $\operatorname{Sh}(Q / U, U \backslash \mathcal{X})$ with fiber $\mathcal{T}=\lim _{\longleftarrow} U(\mathbb{Q}) \backslash U(\mathbb{C}) \times U\left(\boldsymbol{A}_{f}\right) / K_{U, f}$. Here 
$K_{U, f}$ runs over the set of open compact subgroups of $U\left(\boldsymbol{A}_{f}\right)$. At finite level $K_{f}, \pi_{2}$ is a locally constant torus fibration. It follows just as in [HZ1, (3.2.1)] that

$$
[\mathcal{W}]=\pi_{2}^{*}\left(\pi_{2, *}([\mathcal{W}])^{\mathcal{T}}\right) .
$$

Here $\mathcal{T}$ acts naturally on the direct image $\pi_{2, *}([\mathcal{W}])$ (cf. [SGA 3]) and the superscript

$\mathcal{T}$ designates the subsheaf of $\mathcal{T}$-invariant sections.

This can be globalized, following [HZ1, § 4.6]. The subgroup $W \subset Q$ acts freely on the principal bundle $I(Q, X)$. We let $I_{2}(Q, X)$ (resp. $I_{1}(Q, X)$ ) denote the quotient of $I(Q, X)$ by $U$ (resp. by $W)$. Then $I_{2}(Q, X)$ (resp. $I_{1}(Q, X)$ ) represents the functor defined by analogy with (1.2.10), in which the faithful representation $(\rho, L)$ of $Q$ is replaced by a representation of $Q$ that factors through a faithful representation of $Q / U$ (resp. $Q / W$ ). It follows immediately that

$(1.2 .12) \quad I_{2}(Q, X) \stackrel{\sim}{\longrightarrow} \pi_{2}^{*}(I(Q / U, U \backslash X)), \quad I_{1}(Q, X) \stackrel{\sim}{\longrightarrow}\left(\pi_{1} \circ \pi_{2}\right)^{*}(I(Q / W, W \backslash X))$,

the isomorphisms being canonical and, in particular, defined over $E(Q, X)$.

On the other hand, there are canonical isomorphisms

$$
U \backslash \check{x} \stackrel{\sim}{\longrightarrow}(U \backslash X)^{\vee}, \quad W \backslash \check{X} \stackrel{\sim}{\longrightarrow}(W \backslash X)^{\vee} .
$$

The isomorphisms (1.2.13) can be defined over $\mathbb{C}$ tautologically, in terms of the definition of $\check{X}$ as a homogeneous space under $Q(\mathbb{C})$; it is then easy to see that the isomorphisms descend to $E(Q, X)$. Now any $Q$-equivariant vector bundle $\mathcal{W}$ on $\check{X}$ is necessarily $U$-equivariant. Since $U$ acts freely on $\check{X}$, the natural map $\check{\pi}_{2}: \check{X} \rightarrow(U \backslash X)^{\vee}$ makes $\check{X}$ a $U$-torsor over $(U \backslash X)^{\vee}$. It follows that

$$
\mathcal{W}=\check{\pi}_{2}^{*}\left(\mathcal{W}_{U}\right),
$$

for some $Q / U$-homogeneous vector bundle $\mathcal{W}_{U}$ on $(U \backslash X)^{\vee}$. It is then easy to see that, in the notation of (1.2.11),

$$
\pi_{2, *}([\mathcal{W}])^{\mathcal{T}} \stackrel{\sim}{\longrightarrow}\left[\mathcal{W}_{U}\right] .
$$

In particular, $\pi_{2, *}([\mathcal{W}])^{\mathcal{T}}$ is an automorphic vector bundle over $\operatorname{Sh}(Q / U, U \backslash X)$.

The analogue for $\pi_{1}$ is more complicated and will be discussed in $\S 1.4$.

REMARK 1.2.15. - The generalization of Langlands' conjecture on conjugation of Shimura varieties to the setting of mixed Shimura varieties has been carried out in [W1, II, 5]. Wildeshaus also constructs flat automorphic vector bundles on mixed Shimura varieties $[\mathrm{W} 1, \mathrm{II}, 6]$ and determines their behavior with respect to the action of $\operatorname{Aut}(\mathbb{C})$.

1.2.16. Let $x \in X$ and let $Q_{x}^{0} \subset Q$ be its stabilizer, as in $\S 1$. In the diagram (1.2.7), the homogeneous vector bundle $\beta^{*}(\mathcal{W})$ is determined by the isotropy representation of $Q_{x}^{0}$ on the fiber $\mathcal{W}_{x}$. We say $\mathcal{W}$ is fully decomposed if the isotropy representation at some point $x$ is trivial on the unipotent radical $R_{u} Q_{x}^{0}$ of $Q_{x}^{0}$. This condition 
is evidently independent of the choice of $x$, and we then say that the automorphic vector bundle $[\mathcal{W}]$ is fully decomposed.

We will need a more general condition in practice. As above, we write $W=W_{-1} Q$, $U=W_{-2} Q$.

Definition 1.2.16.1. - We say $\mathcal{W}$ satisfies a Kostant condition at $x$ if there are

(i) a reductive group $K_{x}$,

(ii) a parabolic subgroup $\Pi_{x} \subset K_{x}$,

(iii) an injective map $\alpha_{x}: Q_{x}^{0} \cap W \rightarrow \Pi_{x}$ with $\alpha\left(\operatorname{Lie}\left(Q_{x}^{0} \cap W\right)\right)=R_{u} \operatorname{Lie}\left(\Pi_{x}\right)$,

(iv) a finite-dimensional representation $\sigma_{x}$ of $K_{x}$ on $\mathcal{W}_{x}$

such that the isotropy representation of $\operatorname{Lie}\left(Q_{x}^{0} \cap W\right)$ on $\mathcal{W}_{x}$ is equivalent to $\left.\sigma_{x}\right|_{\operatorname{Lie}\left(\Pi_{x}\right)} \circ \alpha_{x}$.

Again, this condition is independent of the choice of $x$, so we simply say that the automorphic vector bundle $[\mathcal{W}]$ satisfies a Kostant condition. Such a condition serves to provide the splitting of spectral sequences at $E_{2}$, as in Corollary 1.4.5 below. This splitting is canonical, given $x$ and items (1.2.16.1,(i)-(iv)) ( $x$ determines a canonical Levi subgroup of $Q)$. Indeed, the derived functor $R \operatorname{Hom}_{\operatorname{Lie}\left(Q_{x}^{0} \cap W\right)}\left(\mathbb{C}, \mathcal{W}_{x}\right)$ splits canonically as the sum of its cohomology objects, for one then gets an embedding

$$
H^{\bullet}\left(\operatorname{Lie}\left(Q_{x}^{0} \cap W\right), \mathcal{W}_{x}\right) \hookrightarrow C^{\bullet}\left(\operatorname{Lie}\left(Q_{x}^{0} \cap W\right), \mathcal{W}_{x}\right),
$$

where $C \bullet$ is the standard Lie algebra cochain complex and $H \bullet$ has the zero differential, inducing the identity on cohomology.

REMARK 1.2.16.3. - The consequence (1.2.16.2) of the Kostant condition is really just $[\mathrm{K},(5.7 .4)]$. In its original form, it was tacitly invoked several times in the calculation of boundary cohomology of the pure Shimura variety $\operatorname{Sh}(G, X)$ in $[\mathrm{HZ} 1, \S 3]$. (To be specific, it enters there in at least 3.5.12, 3.7.8 via 3.7.5, 3.13.4.5, and 3.14.2though not 3.5.8.) The mixed Shimura varieties there were of the form $\left(P_{F}^{\prime}, X\left(D_{F}\right)\right)$ attached to a rational boundary component $F$ of $X$, with $P_{F}^{\prime}=G_{h, F} W_{F}$ and $X\left(D_{F}\right)$ an enlarged Siegel domain of the third kind containing $X$ (see [HZ1, 1.2.3] for this notation). We write $W=W_{F}, U=U_{F}$. If $x \in X$ is viewed as a point in $X\left(D_{F}\right)$, then the natural map

$$
Q_{x}^{0} \cap W \longrightarrow W / U=\mathrm{gr}_{-1} W
$$

is injective and identifies $\operatorname{Lie}\left(Q_{x}^{0} \cap W\right)$ with the anti-holomorphic tangent space $\mathfrak{v}_{x}^{-}$ $[$ HZ1,1.8.4] of the abelian variety corresponding to the point $x$. In this case, the group $K_{x}$ is the complexification of the connected stabilizer of $x \in X$ in $G(\mathbb{R})$; i.e., of the maximal compact (mod center) subgroup of $G(\mathbb{R})$, the parabolic subgroup $\Pi_{x}$ is the group denoted $Q_{F, x}$ in [HZ1,1.8.7], and $\operatorname{Lie}\left(R_{u} Q_{F, x}\right)=\mathfrak{s}_{x}$; and the map $\alpha_{x}$ : $\mathfrak{v}_{x}^{-} \rightarrow \operatorname{Lie}\left(R_{u} Q_{F, x}\right)$ is defined via the Cayley transform in [HZ1,3.5]. In [HZ1: 3.5.12], the embedding (1.2.16.2) is tacitly used, though it might have been more helpful to quote [K: 5.7.4] explicitly. 
1.2.16.4. Variant. - Let $S_{x} \subset Q_{x}^{0} \cap W$ be a subgroup with $\mathfrak{s}_{x}=\operatorname{Lie}\left(S_{x}\right)$. Suppose that the analogues of $\left(1.2 .16 .1\right.$, (i)-(iv)) hold, with $Q_{x}^{0} \cap W$ replaced by $S_{x}$ and $\operatorname{Lie}\left(Q_{x}^{0} \cap W\right)$ replaced by $\mathfrak{s}_{x}$. We then say $[\mathcal{W}]$ satisfies a Kostant condition relative to $S_{x},\left(\right.$ or $\left.\mathfrak{s}_{x}\right)$. The analogue of $(1.2 .16 .2)$ then holds for $\mathfrak{s}_{x}$ : there is a canonical splitting

$$
H^{\bullet}\left(\mathfrak{s}_{x}, \mathcal{W}_{x}\right) \longleftrightarrow C^{\bullet}\left(\mathfrak{s}_{x}, \mathcal{W}_{x}\right) .
$$

A condition of this kind occurs in the proof of Proposition 1.5.24.

1.2.A. Appendix: Periods and rationality. — The articles [H1] and [M1] define canonical rational structures on automorphic vector bundles in terms of $\mathrm{CM}$ motives. This construction is adapted in section 4.3 of [HZ1] to the mixed Shimura varieties treated there. We briefly show how this construction extends to general mixed Shimura varieties.

In the previous version of the present article, the construction of standard principal bundles on mixed Shimura varieties was reduced to the case of pure Shimura varities, by the procedure outlined in the proof of Proposition 1.2.4. We are grateful to the referee for pointing out that the literature only considers pure Shimura varieties in the standard definition, and not the more general definition due to Pink. The purpose of the present appendix is to fill this gap in the literature with an exposition of Pink's constructions. As explained in (1.1.7), it suffices to treat the case of a pure Shimura datum $(T, \mathcal{Y})$, where $T$ is a $\mathbb{Q}$-split torus. However, it is no more difficult to treat a general torus, and we do so now.

If $(Q, \mathcal{X})$ is a general mixed Shimura datum, and if $\left(x, q_{f}\right) \in \mathcal{X} \times Q\left(\boldsymbol{A}_{f}\right)$, we let $\left[x, q_{f}\right]$ denote the corresponding point in $\operatorname{Sh}(Q, \mathcal{X})(\mathbb{C})$. We begin by recalling the period formalism, as described in $[\mathrm{HZ} 1, \S 4.3]$. First suppose $(T, h)$ is a Shimura datum with $T$ a torus and $h$ a point. We write $\check{h}$ for the "compact dual", as above. Let $\mathcal{W}$ be a $T$-homogeneous vector bundle on $\breve{h}$, and let $[\mathcal{W}]$ be the corresponding automorphic vector bundle. The homogeneous vector bundle $\mathcal{W}$ defines a representation $\sigma=\sigma_{\mathcal{W}}$ of $T$ and a period element

$$
p(h, \sigma)=p\left(h, \sigma_{\mathcal{W}}\right) \in \operatorname{Aut}\left(\left.[\mathcal{W}]\right|_{\operatorname{Sh}(T, h)}(\mathbb{C})\right),
$$

well-defined modulo $\sigma_{\mathcal{W}}(T(\mathbb{Q}))$, that compares the de Rham and Betti rational structures of $\left.[\mathcal{W}]\right|_{\operatorname{Sh}(T, h)}(\mathbb{C})$. In the notation of $[\mathrm{HZ1},(4.3 .6)]$, we have

$$
H_{\mathrm{DR}}(\mathfrak{M}(\sigma) / \operatorname{Sh}(T, h))_{\left[h, q_{f}\right]}=p(h, \sigma) \cdot H_{\mathrm{B}}(\mathfrak{M}(\sigma) / \operatorname{Sh}(T, h))_{\left[h, q_{f}\right]} .
$$

Here $q_{f}$ is a variable point in $T\left(\boldsymbol{A}_{f}\right)$, as above.

We note that either side of (1.2.A.1) can be taken to be defined independently. Over $\mathbb{C}$, we just have

$$
H_{\mathrm{B}}(\mathfrak{M}(\sigma) / \operatorname{Sh}(T, h))(\mathbb{C})=\Gamma(\operatorname{Sh}(T, h)(\mathbb{C}), \mathcal{W}(\mathbb{C})),
$$

with $\mathcal{W}(\mathbb{C})$ defined by the formula (1.2.7). There is a reflex field $E(\mathcal{W}) \supset E(T, h)$, such that both sides of (1.2.A.1) have natural $E(\mathcal{W})$-rational structures. (In practice, 
we can take $E(\mathcal{W})$ to be the composite of $E(T, h)$ with a field of definition of the algebraic representation $\sigma$.) Then $H_{\mathrm{B}}(\mathfrak{M}(\sigma) / \operatorname{Sh}(T, h))$ can be defined, in terms of (1.2.A.2), by

$$
H_{\mathrm{B}}(\mathfrak{M}(\sigma) / \operatorname{Sh}(T, h))=\Gamma(\operatorname{Sh}(T, h)(\mathbb{C}), \mathcal{W}(E(\mathcal{W}))),
$$

where by $\mathcal{W}(E(\mathcal{W}))$ we mean the local system in $E(\mathcal{W})$-vector spaces

$$
\left.\lim _{K_{f}} T(\mathbb{Q}) \backslash \mathcal{W}(E(\mathcal{W})) \times\left(T\left(\boldsymbol{A}_{f}\right) / K_{f}\right)\right)
$$

On the other hand, the left-hand side of (1.2.A.1) is given (a posteriori) by

$$
H_{\mathrm{DR}}(\mathfrak{M}(\sigma) / \operatorname{Sh}(T, h))=\Gamma(\operatorname{Sh}(T, h),[\mathcal{W}]),
$$

where here the relevant structures are the $E(T, h)$-scheme $\operatorname{Sh}(T, h)$ and the (rational) vector bundle $[\mathcal{W}]$. The content of (1.2.A.1) is that there exists a theory of period invariants $p(h, \sigma)$ that behaves naturally with respect to morphisms of Shimura data and tensor products. These invariants, initially introduced (up to algebraic factors) by Shimura, are provided by the theory of abelian varieties with complex multiplication, and are described in detail in [H1].

Now let $(T, \mathcal{Y})$ be a general Shimura datum, in Pink's sense, with $T$ a torus; as always we assume hypothesis (1.2.1). We write $h$ for the singleton $\mathcal{H}_{\mathcal{Y}}$; thus there is a morphism of Shimura data $(T, \mathcal{Y}) \rightarrow(T, h)$, inducing a morphism of Shimura varieties $\eta: \operatorname{Sh}(T, \mathcal{Y}) \rightarrow \operatorname{Sh}(T, h)$ as well as a $T$-equivariant identification of compact duals $\check{\mathcal{Y}} \rightarrow \breve{h}$. If $\mathcal{W}$ is a $T$-homogeneous vector bundle on $\check{\mathcal{Y}}=\check{h}$, then we define the corresponding automorphic vector bundle $[\mathcal{W}]_{\mathcal{Y}}$ on $\operatorname{Sh}(T, \mathcal{Y})$ by

$$
[\mathcal{W}]_{\mathcal{Y}}=\eta^{*}[\mathcal{W}]
$$

Here $[\mathcal{W}]$ on the right-hand side is the automorphic vector bundle on $\operatorname{Sh}(T, h)$. This is the only definition compatible with the functoriality assertion of Proposition 1.2.8. The element $p(h, \sigma)$ of (1.2.A.1) lifts to an automorphism of $[\mathcal{W}]_{\mathcal{Y}}$ which we denote $p(h, \sigma) \mathcal{y}$. We then tautologically obtain

$$
H_{\mathrm{DR}}(\mathfrak{M}(\sigma) / \operatorname{Sh}(T, \mathcal{Y}))_{\left[y, q_{f}\right]}=p\left(h, \sigma_{\mathcal{Y}}\right) \cdot H_{\mathrm{B}}(\mathfrak{M}(\sigma) / \operatorname{Sh}(T, \mathcal{Y}))_{\left[y, q_{f}\right]} .
$$

for $y \in \mathcal{Y}$, where both sides of (1.2.A.6) are defined in terms of the corresponding sides of (1.2.A.1) by pullback.

This apparently simplistic definition of the period invariant has a simple justification. There is an isomorphism

$$
\Gamma\left(\operatorname{Sh}(T, \mathcal{Y}),[\mathcal{W}]_{\mathcal{Y}}\right) \stackrel{\sim}{\longrightarrow} \mathcal{O}_{\operatorname{Sh}(T, \mathcal{Y})} \otimes_{\mathcal{O}(\operatorname{Sh}(T, h))} \Gamma(\operatorname{Sh}(T, h),[\mathcal{W}]) .
$$

The period term is concentrated in the second factor on the right; the first factor accounts for the reciprocity law of $\operatorname{Sh}(T, \mathcal{Y})$, as defined by Pink.

Now we consider the period invariant in general. Let $(Q, X)$ be a mixed Shimura datum. Let $\mathcal{W}$ be a $Q$-homogeneous vector bundle on $\check{x}$, and let $[\mathcal{W}]$ be the corresponding automorphic vector bundle. As in the pure case, there is a canonical 
"periods" isomorphism

$$
\operatorname{Per}_{\left(x, q_{f}\right)}: \mathcal{W}_{\beta(x)} \stackrel{\sim}{\longrightarrow}[\mathcal{W}]_{\left[x, q_{f}\right]}
$$

(cf. [HZ1, (4.3.5)]). Suppose $\mathcal{W}$ is rational over the extension $E(\mathcal{W})$ of $E(Q, X)$, as $Q$-homogeneous vector bundle. By Proposition 1.2.8, $[\mathcal{W}]$ has a canonical model over $E(\mathcal{W})$. This model has the following description in terms of the period elements: For any map $(T, \mathcal{Y}) \hookrightarrow(Q, \mathcal{X})$, with $T$ a torus, let $E(T, \mathcal{Y} ; \mathcal{W})=E(T, \mathcal{Y}) \cdot E(\mathcal{W})$. Then for any field $L \supset E(T, \mathcal{Y} ; \mathcal{W}), x \in \mathcal{Y}$, and any $t_{f} \in T\left(\boldsymbol{A}_{f}\right)$, we have

$$
\left.[\mathcal{W}](L)\right|_{\left[x, t_{f}\right]}=\left.p\left(h, \sigma_{\mathcal{W}}\right) \cdot \operatorname{Per}_{\left(x, t_{f}\right)}\left(\mathcal{W}_{\beta(x)}(L)\right) \subset[\mathcal{W}]\right|_{\left[x, t_{f}\right]}(\mathbb{C})
$$

This both summarizes and generalizes the discussion that follows [HZ1, (4.3.6)].

\subsection{Canonical extensions of automorphic vector bundles}

Let ${ }_{K_{f}} \operatorname{Sh}(Q, X) \subset{ }_{K_{f}} \operatorname{Sh}(Q, X)_{\Sigma}$ be a (partial) toroidal compactification as an algebraic variety, as in (1.1.6). We retain Hypothesis (1.2.1) and assume for the remainder of $\S 1$ that $K_{f}$ is neat, in the sense of $[\mathrm{P}, 0.6]$, and that $K_{f} \mathrm{Sh}(Q, \mathcal{X})_{\Sigma}$ is an SNC compactification. We write $\mathrm{Sh}={ }_{K_{f}} \operatorname{Sh}(Q, X), \mathrm{Sh}_{\Sigma}=K_{f} \operatorname{Sh}(Q, X)_{\Sigma}, \partial \mathrm{Sh}_{\Sigma}=\partial_{K_{f}} \operatorname{Sh}(Q, X)_{\Sigma}$.

After (1.3.5), and for the remainder of the paper, we also assume $K_{f} \operatorname{Sh}(Q, X)_{\Sigma}$ to be compact, unless an explicit statement is made to the contrary.

Our hypothesis that $K_{f}$ is neat implies, as in the pure case (cf. the proof of $\left[\mathrm{H} 2\right.$, Theorem 4.2]) that the connection $\nabla_{L}$ has unipotent monodromy. Deligne's existence theorem [De1] thus provides a unique extension of $\widetilde{L}$ to a vector bundle $\widetilde{L}_{\Sigma}$ over $\mathrm{Sh}_{\Sigma}$ such that $\nabla$ extends to a connection $\nabla_{\Sigma}$ with regular singularities and nilpotent connection forms along $\partial \mathrm{Sh}_{\Sigma}$.

Definition 1.3.1. - Let $[\mathcal{W}]$ be an automorphic vector bundle on Sh. An admissible metric on $[\mathcal{W}]$ is a hermitian metric whose pullback, under the natural map $\mathcal{X} \times Q\left(\boldsymbol{A}_{f}\right) \rightarrow$ Sh, is a $Q(\mathbb{R}) \cdot U(\mathbb{C}) \times Q\left(\boldsymbol{A}_{f}\right)$-invariant hermitian metric on $\beta^{*}\left(\mathcal{W} \times \mathcal{X} \times Q\left(\boldsymbol{A}_{f}\right)\right)$.

The stabilizer $\operatorname{Stab}(x)$ in $Q(\mathbb{R}) \cdot U(\mathbb{C})$ of any point $x \in X$ is a compact Lie group (Pink doesn't state this explicitly, but it is clear from Lemma 1.17 of [P]; cf. the proof of [P, Prop. 2.11].). Therefore, admissible metrics exist and their restrictions to indecomposable $C^{\infty}$ summands of $[\mathcal{W}]$ are unique up to constant multiples. Note that the automorphic vector bundles that are indecomposable as $C^{\infty}$ automorphic bundles are parametrized by irreducible representations of the compact group $\operatorname{Stab}(x)$, for one (or any) $x \in \mathcal{X}$. The indecomposable holomorphic automorphic bundles are parametrized by locally-finite indecomposable representations of the algebraic subgroup $Q_{x}^{0} \subset Q$, defined in (1.1). 
LEMMA 1.3 .2

(i) Let $[\mathcal{W}]$ be an automorphic vector bundle on $\mathrm{Sh}$. Then $[\mathcal{W}]$ has an admissible metric.

(ii) Let $h(\cdot, \cdot)$ be an admissible metric on the flat vector bundle $\widetilde{L}$. Let $B \subset \mathrm{Sh}_{\Sigma}$ be an open polydisk with coordinates $z_{1}, \ldots z_{n}$ chosen so that $B \cap \partial \mathrm{Sh}_{\Sigma}$ is the union of the coordinate hyperplanes defined by $z_{1}, \ldots, z_{r}$ for some $r \leqslant n$. Then

$$
\begin{array}{r}
\Gamma\left(B, \widetilde{L}_{\Sigma}\right)=\left\{s \in \Gamma(B \cap \operatorname{Sh}, \widetilde{L})|| h(s, s) \mid=O\left(P\left(|\log | z_{1}||, \ldots,|\log | z_{r}||\right)\right)\right. \\
\text { for some polynomial } P\} .
\end{array}
$$

Proof. - Part (i) has already been explained. To prove Part (ii), we reduce to the case of an equivariant torus embedding. Indeed, let $Q^{\prime} \subset Q$ be a rational boundary subgroup and let $X^{\prime}$ be the corresponding rational boundary component of $X$, in the sense of $[\mathrm{P}, \S 4]$. We let $U^{\prime} \subset W^{\prime}=R_{u} Q^{\prime} \subset Q^{\prime}$ be the canonical filtration of $Q^{\prime}$, and let $\pi_{2}^{\prime}: \operatorname{Sh}\left(Q^{\prime}, X^{\prime}\right) \rightarrow \operatorname{Sh}\left(Q^{\prime} / U^{\prime}, U^{\prime} \backslash X^{\prime}\right)$ be the canonical map, representing $\operatorname{Sh}\left(Q^{\prime}, X^{\prime}\right)$ as the locally constant pro-torus fibration over $\operatorname{Sh}\left(Q^{\prime} / U^{\prime}, U^{\prime} \backslash X^{\prime}\right)$ with structure group $\mathcal{T}^{\prime}$, as in the discussion preceding (1.2.11). There is a natural embedding $\check{X}^{\prime} \subset \check{X}$ (cf. [HZ1, Lemma 4.5.8]), extending the inclusion of $\beta\left(X^{\prime}\right)$ in $\check{X}$ as a Siegel domain of the third kind. There is also a neighborhood $\operatorname{Sh}\left(Q^{\prime}, X^{\prime}\right)_{\Sigma_{Q^{\prime}}}^{+}$of the boundary $\partial \operatorname{Sh}\left(Q^{\prime}, X^{\prime}\right)_{\Sigma_{Q^{\prime}}}$ admitting a natural map

$$
f_{Q^{\prime}}: \operatorname{Sh}\left(Q^{\prime}, X^{\prime}\right)_{\Sigma_{Q^{\prime}}}^{+} \longrightarrow \operatorname{Sh}_{\Sigma}
$$

The map $f_{Q^{\prime}}$ is a local isomorphism in a neighborhood of the part of the boundary of $\mathrm{Sh}_{\Sigma}$ corresponding to $Q^{\prime}$.

We assume $Q^{\prime}$ chosen so that the polydisk $B$ lies in the image of $f_{Q^{\prime}}$. The flat vector bundle $\widetilde{L}$ is defined by the representation $\rho$, say of $Q$. The restriction of $\rho$ to $Q^{\prime}$ defines a flat vector bundle $(\widetilde{L})^{\prime}$ on $\operatorname{Sh}\left(Q^{\prime}, X^{\prime}\right)$ whose restriction to $\operatorname{Sh}\left(Q^{\prime}, X^{\prime}\right)_{\Sigma_{Q^{\prime}}}^{+} \cap \operatorname{Sh}\left(Q^{\prime}, X^{\prime}\right)$ is equivalent to $f_{Q^{\prime}}^{*}(\widetilde{L})$. Then it is clear by uniqueness that the admissible metric $h(\cdot, \cdot)$ on $\widetilde{L}$ pulls back to the restriction to $\operatorname{Sh}\left(Q^{\prime}, X^{\prime}\right)_{\Sigma_{Q^{\prime}}}^{+}$of an admissible metric on $(\widetilde{L})^{\prime}$. Thus we may assume $Q^{\prime}=Q$ and that $B$ is not contained in the image of $f_{Q^{\prime \prime}}$ for any proper boundary subgroup $Q^{\prime \prime}$ of $Q$. In other words, possibly shrinking $B$, the map $\pi_{2}$ can be written as a product

$$
\pi_{2}: B \stackrel{\sim}{\longrightarrow} B_{0} \times \mathcal{T}_{\Sigma(\mathcal{T})}
$$

where $B_{0}$ is open in $\operatorname{Sh}(Q / U, U \backslash \mathcal{X})$ and $\mathcal{T} \hookrightarrow \mathcal{T}_{\Sigma(\mathcal{T})}$ is an equivariant torus embedding, with $\mathcal{T}$ a torus of the form $U(\mathbb{Q}) \backslash U(\mathbb{C}) \times U\left(\boldsymbol{A}_{f}\right) / K_{U, f}$ as in the discussion at the end of $\S 1.2$. Near $B$ the local system is thus defined by a representation of the fundamental group of $\mathcal{T}$. In this way, we may assume $Q=U$, Sh is a torus of the form $U(\mathbb{C}) / \Lambda$, for some lattice $\Lambda$ in $U(\mathbb{R}), \widetilde{L}$ is the local system defined by a representation $(\rho, L)$ of $\Lambda$, and $h(\cdot, \cdot)$ is a $U(\mathbb{C})$-invariant metric. The representation $\rho$ is the restriction to $\Lambda$ of a unipotent representation of the algebraic group $U$. Moreover, we may assume $n=r$ 
and $\mathrm{Sh} \hookrightarrow \mathrm{Sh}_{\Sigma}$ is isomorphic to the obvious embedding $\mathbb{C}^{\times, n} \rightarrow \mathbb{C}^{n}$, with coordinates $z_{1}, \ldots, z_{n}$. In particular, $\mathrm{Sh}_{\Sigma}$ is affine, hence $\widetilde{L}_{\Sigma}$ generated by its global sections.

Let $j: U(\mathbb{C}) \times U\left(\boldsymbol{A}_{f}\right) \rightarrow$ Sh denote the natural map. We fix a basepoint, say $o$, in $U(\mathbb{C})$, and a basis $\left\{e_{1}, \ldots, e_{N}\right\}$ of $L$, such that $h\left(e_{i}, e_{j}\right)_{o}$ is the identity matrix. It follows from the $U(\mathbb{C})$-invariance of $h(\cdot, \cdot)$ that, with respect to the trivialization $j^{*}(\widetilde{L}) \stackrel{\sim}{\longrightarrow} U(\mathbb{C}) \times L$, the matrix of $h\left(e_{i}, e_{j}\right)_{u}$ is polynomial in the variable $u \in U(\mathbb{C})$, hence has logarithmic growth in terms of the coordinates $z_{1}, \ldots, z_{n}$. Now let $\left\{v_{1}, \ldots, v_{N}\right\}$ denote a basis of global sections of $\widetilde{L}_{\Sigma}$, and express the $v_{i}$ in terms of the horizontal sections $e_{j}$ over the universal cover $U(\mathbb{C})$ of Sh:

$$
v_{i}=\sum_{j} f_{i j} e_{j}
$$

where $f_{i j}$ are functions on $U(\mathbb{C})$. Since the monodromy of $\widetilde{L}$ is unipotent, the $f_{i j}$ have logarithmic growth in terms of $z_{1}, \ldots, z_{n}$. The inclusion of the left-hand side of (1.3.3) in the right-hand side is now clear. The opposite inclusion then follows because any section satisfying an inequality of the indicated form has removable singularities with respect to the basis $\left\{v_{1}, \ldots, v_{N}\right\}$.

A local section of $\widetilde{L}$ satisfying an inequality of type (1.3.3) is said to be slowly increasing.

In (ii) of the following Definition-Proposition, we let $\left(Q^{\prime}, X^{\prime}\right), U^{\prime}$, and so on be as in the proof of Lemma 1.3.2.

Definition-Proposition 1.3.5. - Let $[\mathcal{W}]$ be an automorphic vector bundle on Sh. A canonical extension $[\mathcal{W}]_{\Sigma}$ of $[\mathcal{W}]$ over $\mathrm{Sh}_{\Sigma}$ is a vector bundle over $\mathrm{Sh}_{\Sigma}$ satisfying one of the following equivalent conditions:

(i) If $h(\cdot, \cdot)$ is an admissible metric on [W] and if $B$ is as in Lemma (1.3.2)(ii), then

$$
\begin{array}{r}
\Gamma\left(B,[\mathcal{W}]_{\Sigma}\right)=\left\{s \in \Gamma(B \cap \operatorname{Sh},[\mathcal{W}])|| h(s, s) \mid=O\left(P\left(|\log | z_{1}||, \ldots,|\log | z_{r}||\right)\right)\right. \\
\text { for some polynomial } P\}
\end{array}
$$

the polynomial $P$ above may depend on $s$.

(ii) Let $\left(Q^{\prime}, X^{\prime}\right)$ be a rational boundary pair for $(Q, \mathcal{X})$, as above. Write $\Sigma=$ $\cup \Sigma_{P}$, the union taken over the rational boundary subgroups $P$ of $Q$. If $\sigma \in \Sigma_{Q^{\prime}}$, let $\operatorname{Sh}\left(Q^{\prime}, X^{\prime}\right)_{\sigma} \subset \operatorname{Sh}\left(Q^{\prime}, X^{\prime}\right)_{\Sigma_{Q^{\prime}}}$ denote the corresponding partial compactification, and let

$$
\pi_{2, \sigma}^{\prime}: \operatorname{Sh}\left(Q^{\prime}, X^{\prime}\right)_{\sigma} \longrightarrow \operatorname{Sh}\left(Q^{\prime} / U^{\prime}, U^{\prime} \backslash X^{\prime}\right)
$$

denote the natural extension of $\pi_{2}^{\prime}$ (cf. $[\mathrm{H} 2,4.1]$ for the analogue in the pure case). Let $[\mathcal{W}]^{\prime}$ denote the automorphic vector bundle on $\operatorname{Sh}\left(Q^{\prime}, X^{\prime}\right)$ associated to the $Q^{\prime}$ homogeneous vector bundle $\mathcal{W}$, restricted to $\check{X}^{\prime} \subset \check{X}$. Then via the natural map (1.3.4) 
we have canonical isomorphisms

$$
\phi_{\sigma}: f_{Q^{\prime}}^{*}\left([\mathcal{W}]_{\Sigma}\right) \stackrel{\sim}{\longrightarrow}\left(\pi_{2, \sigma}^{\prime}\right)^{*}\left(\pi_{2, *}^{\prime}\left([\mathcal{W}]^{\prime}\right)^{\mathcal{T}^{\prime}}\right),
$$

and these isomorphisms are compatible with respect to inclusions $\sigma \subset \tau$.

(iii) There is a flat automorphic vector bundle $\widetilde{L}$ such that $[\mathcal{W}]$ can be realized as an automorphic subquotient of $\widetilde{L}$, and $[\mathcal{W}]_{\Sigma}$ is a locally free subquotient of the Deligne extension $\widetilde{L}_{\Sigma}$, compatibly with the given realization over $\mathrm{Sh}$.

The canonical extension $[\mathcal{W}]_{\Sigma}$ exists and is unique.

Proof. - The existence of $[\mathcal{W}]_{\Sigma}$ satisfying (iii) is proved in exactly the same way as the corresponding assertion for pure Shimura varieties [H2, Theorem 4.2]. The equivalence of (iii) with (ii), similarly, is identical to the proof of (4.2.2) of [H2].

Let $B$ be as in (i). It follows from Lemma $(1.3 .2)\left(\right.$ ii) that, if $[\mathcal{W}]_{\Sigma}$ satisfies (iii), then there is an inclusion

$$
\Gamma\left(B,[\mathcal{W}]_{\Sigma}\right) \subset\{s \in \Gamma(B \cap \mathrm{Sh},[\mathcal{W}]) \mid s \text { is slowly increasing }\} .
$$

But then every $s \in \Gamma(B \cap \mathrm{Sh},[\mathcal{W}])$ in the right-hand side of (1.3.6) has a removable singularity along $\partial \mathrm{Sh}_{\Sigma}$ and therefore extends to an element of $\Gamma\left(B,[\mathcal{W}]_{\Sigma}\right)$. This shows the equivalence of (iii) with (i). Uniqueness is clear from (i).

As mentioned at the beginning of this section, unless an explicit statement is made to the contrary, we now assume our partial compactifications $\mathrm{Sh}_{\Sigma}$ to be compact, as well as SNC. Then $\partial \mathrm{Sh}_{\Sigma}$ is a complete divisor with normal crossings on $\mathrm{Sh}_{\Sigma}$, defined by the invertible sheaf of ideals $\mathcal{I}_{\partial \mathrm{Sh}_{\Sigma}}$. If $[\mathcal{W}]$ is an automorphic vector bundle on $\mathrm{Sh}$ with canonical extension $[\mathcal{W}]_{\Sigma}$, we define the subcanonical extension of $[\mathcal{W}]$ to be

$$
[\mathcal{W}]^{\text {sub }}=[\mathcal{W}]_{\Sigma}^{\text {sub }}=[\mathcal{W}]_{\Sigma} \otimes \mathcal{I}_{\partial \mathrm{Sh}_{\Sigma}}
$$

the subscript $\Sigma_{\Sigma}$ will be omitted whenever possible. Similarly, we will write $[\mathcal{W}]^{\text {can }}$ in place of $[\mathcal{W}]_{\Sigma}$ when $\Sigma$ is understood.

The theory of coherent cohomology developed in [H3, H4] extends without change to the case of mixed Shimura varieties. We state the main results in the present context.

Proposition 1.3.7. - Let $(Q, \mathcal{X})$ be a mixed Shimura datum, and let $[\mathcal{W}]$ be an automorphic vector bundle on $\mathrm{Sh}={ }_{K_{f}} \mathrm{Sh}={ }_{K_{f}} \mathrm{Sh}(Q, X)$, for some open compact subgroup $K_{f}$ of $Q\left(\boldsymbol{A}_{f}\right)$. Let $\mathrm{Sh}_{\Sigma}$ be a toroidal compactification of Sh.

(i) Let $\Sigma_{1}$ be a refinement of $\Sigma$, and let $f_{\Sigma_{1}, \Sigma}: \mathrm{Sh}_{\Sigma_{1}} \rightarrow \mathrm{Sh}_{\Sigma}$ be the corresponding morphism of compactifications. Then there are isomorphisms

$$
f_{\Sigma_{1}, \Sigma}^{*}\left([\mathcal{W}]_{\Sigma}\right) \stackrel{\sim}{\longrightarrow}[\mathcal{W}]_{\Sigma_{1}}, \quad f_{\Sigma_{1}, \Sigma}^{*}\left([\mathcal{W}]_{\Sigma}^{\text {sub }}\right) \stackrel{\sim}{\longrightarrow}[\mathcal{W}]_{\Sigma_{1}}^{\text {sub }},
$$

functorial in $\mathcal{W}$, and the natural morphisms

$$
\begin{aligned}
& H^{q}\left(\mathrm{Sh}_{\Sigma},[\mathcal{W}]_{\Sigma}\right) \longrightarrow H^{q}\left(\mathrm{Sh}_{\Sigma_{1}},[\mathcal{W}]_{\Sigma_{1}}\right) \\
& H^{q}\left(\mathrm{Sh}_{\Sigma},[\mathcal{W}]_{\Sigma}^{\text {sub }}\right) \longrightarrow H^{q}\left(\mathrm{Sh}_{\Sigma_{1}},[\mathcal{W}]_{\Sigma_{1}}^{\text {sub }}\right)
\end{aligned}
$$


are isomorphisms for all $q$.

(ii) For each q, we define

$$
\widetilde{H}^{q}\left(\mathrm{Sh},[\mathcal{W}]^{\text {can }}\right)=H^{q}\left(\mathrm{Sh}_{\Sigma},[\mathcal{W}]_{\Sigma}\right)
$$

for any $\Sigma$; we can also write

$$
\widetilde{H}^{q}\left(\mathrm{Sh},[\mathcal{W}]^{\text {can }}\right)=\underset{\Sigma}{\lim _{\Sigma}} H^{q}\left(\mathrm{Sh}_{\Sigma},[\mathcal{W}]_{\Sigma}\right),
$$

the limit taken over the system of all $\Sigma$ 's, all maps being isomorphisms. Similarly, we define

$$
\widetilde{H}^{q}\left(\mathrm{Sh},[\mathcal{W}]^{\mathrm{sub}}\right)=\underset{\Sigma}{\lim _{\Sigma}} H^{q}\left(\mathrm{Sh}_{\Sigma},[\mathcal{W}]_{\Sigma}^{\mathrm{sub}}\right)
$$

Let

$$
\begin{aligned}
& \widetilde{H}^{q}\left([\mathcal{W}]^{\text {can }}\right)=\underset{\lim _{K_{f}}}{ } \widetilde{H}^{q}\left(K_{f} \mathrm{Sh},[\mathcal{W}]^{\text {can }}\right), \\
& \widetilde{H}^{q}\left([\mathcal{W}]^{\text {sub }}\right)=\underset{\lim _{f}}{ } \widetilde{H}^{q}\left(K_{f} \text { Sh, }[\mathcal{W}]^{\text {sub }}\right) .
\end{aligned}
$$

Then $\widetilde{H}^{q}\left([\mathcal{W}]^{\text {can }}\right)$ and $\widetilde{H}^{q}\left([\mathcal{W}]^{\text {sub }}\right)$ are naturally admissible $Q\left(\boldsymbol{A}_{f}\right)$-modules.

The proof of Proposition 1.3.7 follows word for word the proofs of Propositions 2.4 and 2.6 of [H4]. The action of $Q\left(\boldsymbol{A}_{f}\right)$ arising in (ii) is defined as in [H4, (2.5.2)]: to any $h \in Q\left(\boldsymbol{A}_{f}\right)$ and any fixed toroidal compactification $K_{f} \operatorname{Sh}(Q, X)_{\Sigma}$ at level $K_{f}$, there is an analogous compactification ${ }_{K_{f}^{h}} \operatorname{Sh}(Q, X)_{\Sigma^{h}}$ of $K_{f}^{h} \operatorname{Sh}(Q, X)$, where $K_{f}^{h}=h^{-1} K_{f} h$. Right-multiplication by $h$ defines an isomorphism $K_{f} \operatorname{Sh}(Q, X)_{\Sigma} \stackrel{\sim}{\longrightarrow} K_{+}^{h} \operatorname{Sh}(Q, X)_{\Sigma^{h}}$ that respects automorphic vector bundles and canonical extensions. By functoriality, we thus obtain an operator $t_{h}$ in the limit on $\widetilde{H}^{q}\left([\mathcal{W}]^{\text {can }}\right)$ and $\widetilde{H}^{q}\left([\mathcal{W}]^{\text {sub }}\right)$.

For any automorphic vector bundle $[\mathcal{W}]$ on Sh we consider the Dolbeault complex $\mathcal{A}^{0, \bullet}([\mathcal{W}])$ on Sh. For each $q, \mathcal{A}^{0, q}([\mathcal{W}])$ will denote the sheaf of $C^{\infty}$-sections of the bundle $\Omega^{0, q} \otimes[\mathcal{W}]$ of $(0, q)$-forms with values in $[\mathcal{W}]$. Letting $j_{\Sigma}$ denote the embedding Sh $\hookrightarrow \mathrm{Sh}_{\Sigma}$, we have, for each $q$, the direct image sheaf $j_{\Sigma, *}\left(\mathcal{A}^{0, q}([\mathcal{W}])\right)$ on $\mathrm{Sh}_{\Sigma}$. We let $\left.\mathcal{A}^{0, q}([\mathcal{W}])\right)_{s i} \subset j_{\Sigma, *}\left(\mathcal{A}^{0, q}([\mathcal{W}])\right)$ denote the subsheaf of sections $s$ such that $s$ and $\bar{\partial} s$ are both slowly increasing, in the sense of (1.3.3), with respect to admissible metrics on the bundles $\Omega^{0, q} \otimes[\mathcal{W}]$ and $\Omega^{0, q+1} \otimes[\mathcal{W}]$. Similarly, we define the rapidly decreasing sections, and the sheaf $\left.\mathcal{A}^{0, q}([\mathcal{W}])\right)_{r d}$, as in [HZ1,3.8.2]. We let $C^{0, q}([\mathcal{W}])_{*}=\varliminf_{K_{f}, \Sigma} \Gamma\left(\mathrm{Sh}_{\Sigma}, \mathcal{A}^{0, q}\left([\mathcal{W}]_{*}\right)\right.$, where * denotes either si or $r d$.

TheOREM 1.3.8. - There is a natural commutative diagram of $Q\left(\boldsymbol{A}_{f}\right)$-modules, where the horizontal maps are isomorphisms:

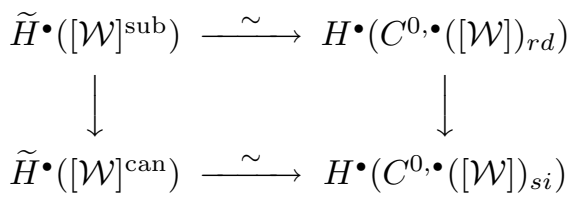


The proof is identical to that of [H4, Corollary 3.4].

A purely algebraic construction of the canonical extension of an automorphic vector bundle was given in [HZ1], in the case of pure Shimura varieties. The analogous construction works here as well.

Proposition 1.3.9. - We retain the notation of Proposition (1.3.7).

(i) The standard principal bundle $I(Q, X)$ extends to an $E(Q, X)$-rational $Q\left(\boldsymbol{A}_{f}\right)$ equivariant principal $Q$-bundle $I(Q, X)_{\Sigma}$ over $\operatorname{Sh}(Q, X)_{\Sigma}$.

(ii) The morphism $p: I(Q, \mathcal{X}) \rightarrow \check{X}$ extends to a Q-equivariant morphism $p_{\Sigma}$ : $I(Q, \mathcal{X})_{\Sigma} \rightarrow \check{X}$, rational over $E(Q, \mathcal{X})$.

(iii) For any automorphic vector bundle $[\mathcal{W}]$ on $\operatorname{Sh}(Q, X)$, there is a canonical isomorphism of vector bundles over $\operatorname{Sh}(Q, X)_{\Sigma}$ :

$$
[\mathcal{W}]_{\Sigma} \stackrel{\sim}{\longrightarrow} p_{\Sigma}^{*}(\mathcal{W}) / Q
$$

This isomorphism is rational over the field of definition of $\mathcal{W}$ as a $Q$-equivariant vector bundle over $\check{X}$. Over $\operatorname{Sh}(Q, X)$ this isomorphism restricts to the construction of $[\mathcal{W}]$ given in $\S 1.2$.

Proof. — The proof is identical to that of Lemma 4.4 .2 of [HZ1].

REMARK 1.3.10. - Just as in Remark (1.2.15), we can formulate a conjecture for the action of $\operatorname{Aut}(\mathbb{C})$ on the canonical extensions of automorphic vector bundles. It should again be a simple matter to reduce this conjecture to the case of pure Shimura varieties, where it is known [BHR, Proposition 1.4.3].

\subsection{Functorial properties of canonical extensions}

We retain the notation of the previous sections, and let

$$
\pi_{2}: \operatorname{Sh}(Q, X) \longrightarrow \operatorname{Sh}(Q / U, U \backslash X) \quad \text { and } \quad \pi_{1}: \operatorname{Sh}(Q / U, U \backslash X) \longrightarrow \operatorname{Sh}(G, W \backslash X)
$$

be the morphisms of (1.1.5). Let $K_{f} \subset Q\left(\boldsymbol{A}_{f}\right)$ be any neat open compact subgroup and let

$$
K_{f}^{2}=K_{f} /\left(U\left(\boldsymbol{A}_{f}\right) \cap K_{f}\right) \subset(Q / U)\left(\boldsymbol{A}_{f}\right), \quad K_{f}^{1}=K_{f} /\left(W\left(\boldsymbol{A}_{f}\right) \cap K_{f}\right) \subset G\left(\boldsymbol{A}_{f}\right) .
$$

Then there are natural morphisms at level $K_{f}$, also denoted $\pi_{2}$ and $\pi_{1}$ :

$$
K_{f} \operatorname{Sh}(Q, X) \stackrel{\pi_{2}}{\longrightarrow} K_{f}^{2} \operatorname{Sh}(Q / U, U \backslash X) \stackrel{\pi_{1}}{\longrightarrow} K_{f}^{1} \operatorname{Sh}(G, W \backslash X) .
$$

Now we can find families of fans $\Sigma, \Sigma_{2}, \Sigma_{1}$, admissible relative to $K_{f}, K_{f}^{2}$, and $K_{f}^{1}$, respectively, such that $K_{f} \operatorname{Sh}(Q, X)_{\Sigma}$ is compact and such that the morphisms in (1.4.1) extend to the corresponding toroidal compactifications $[\mathrm{P}, 6.7(\mathrm{~b})]$, which we assume to be $\mathrm{SNC}$ and rational over $E(Q, X)$ :

$$
K_{f} \operatorname{Sh}(Q, X)_{\Sigma} \stackrel{\pi_{2, \Sigma}}{\longrightarrow} K_{f}^{2} \operatorname{Sh}(Q / U, U \backslash X)_{\Sigma_{2}} \stackrel{\pi_{1, \Sigma}}{\longrightarrow} K_{f}^{1} \operatorname{Sh}(G, W \backslash X)_{\Sigma_{1}}
$$


In what follows, we let $\mathcal{W}$ be a $Q$-homogeneous vector bundle on $\check{x}$ and let $[\mathcal{W}]$ be the corresponding automorphic vector bundle on ${ }_{K_{f}} \operatorname{Sh}(Q, X)$.

Proposition 1.4.3. - With the above notation, the higher direct images

$$
R^{q} \pi_{2, \Sigma, *}[\mathcal{W}]^{\text {can }}=0, \quad R^{q} \pi_{2, \Sigma, *}[\mathcal{W}]^{\text {sub }}=0
$$

for $q>0$, and we have canonical isomorphisms

$$
\begin{aligned}
& \pi_{2, \Sigma, *}[\mathcal{W}]^{\text {can }} \stackrel{\sim}{\longrightarrow}\left(\pi_{2, \Sigma, *}[\mathcal{W}]\right)^{\text {can }} \stackrel{\sim}{\longrightarrow}\left[\mathcal{W}_{U}\right]^{\text {can }} \\
& \pi_{2, \Sigma, *}[\mathcal{W}]^{\text {sub }} \stackrel{\sim}{\longrightarrow}\left(\pi_{2, \Sigma, *}[\mathcal{W}]\right)^{\text {sub }} \stackrel{\sim}{\longrightarrow}\left[\mathcal{W}_{U}\right]^{\text {sub }},
\end{aligned}
$$

in the notation of (1.2.14). Moreover, there is a canonical isomorphism

$$
[\mathcal{W}]^{\text {can }} \stackrel{\sim}{\longrightarrow} \pi_{2, \Sigma}^{*}\left(\pi_{2, \Sigma, *}[\mathcal{W}]\right)^{\text {can }} .
$$

Proof. - The isomorphism (1.4.3.3) is analogous to (i) of [HZ1, Proposition 3.12.2] and is proved in the same way. The vanishing of the higher direct images of $[\mathcal{W}]^{\text {can }}$, together with the first isomorphism in (1.4.3.1), is a mild generalization of (ii) of [HZ1, Proposition 3.12.2]. The proof in the present situation is identical, the basic local ingredient being a calculation [HZ1, Lemma 1.6.8 (iii)] in the setting of proper morphisms of torus embeddings. The second isomorphism in (1.4.3.1) is clear from (1.2.14).

The assertions for subcanonical extensions follow as in [HZ1, (3.14.1)] from the toric version proved in [HZ1, Lemma 1.6.8 (iii)].

The following proposition is more substantial. In what follows, we let $\mathfrak{v}=\operatorname{Lie}(V)$. Any $x \in X$ determines a subalgebra $F_{x}^{0}(\operatorname{Lie}(Q)) \subset \operatorname{Lie}(Q)$, as in (1.1), and we let $\mathfrak{v}_{x}^{-}=F_{x}^{0}(\operatorname{Lie}(Q)) \cap \mathfrak{v}$, as in $[\mathrm{HZ1}, 1.8]$. The (abelian) Lie algebra $\mathfrak{v}_{x}^{-}$depends only on the image $\check{\pi}_{1} \circ \check{\pi}_{2}$ of $x$ in $W \backslash X$, which we denote $\bar{x}$ in the following proposition.

Proposition 1.4.4. - Let $\mathcal{V}$ be a $Q / U$-homogeneous vector bundle on $U \backslash \check{X}$ and let $[\mathcal{V}]$ be the corresponding automorphic vector bundle on $K_{f}^{2} \mathrm{Sh}(Q / U, U \backslash X)$. For any integer $q$, let $\mathcal{V}^{(q)}$ be the $G$-homogeneous vector bundle on $W \backslash \check{X} \simeq(W \backslash X)^{\vee}$ whose fiber at the point $\bar{x}$ is the representation of $F_{\bar{x}}^{0}(\operatorname{Lie}(G))$ on $H^{q}\left(\mathfrak{v}_{x}^{-}, \mathcal{V}_{x}\right)$, for any $x \in \check{X}$. Then for all $q$, there are canonical isomorphisms

$$
\begin{aligned}
& R^{q} \pi_{1, \Sigma, *}[\mathcal{V}]^{\text {can }} \stackrel{\sim}{\longrightarrow}\left(R^{q} \pi_{1, \Sigma, *}[\mathcal{V}]\right)^{\text {can }} \stackrel{\sim}{\longrightarrow}\left[\mathcal{V}^{(q)}\right]^{\text {can }} \\
& R^{q} \pi_{1, \Sigma, *}[\mathcal{V}]^{\text {sub }} \stackrel{\sim}{\longrightarrow}\left(R^{q} \pi_{1, \Sigma, *}[\mathcal{V}]\right)^{\text {sub }} \stackrel{\sim}{\longrightarrow}\left[\mathcal{V}^{(q)}\right]^{\text {sub }}
\end{aligned}
$$

of vector bundles on $K_{f}^{1} \operatorname{Sh}(G, W \backslash X)_{\Sigma_{1}}$. The family of such isomorphisms, as $\mathcal{V}$ varies, is rational over $E(Q, X)$; in other words, the isomorphisms respect canonical models. 
Proof. - We use the characterization of the canonical extensions by growth conditions in (1.3.5)(i), and the calculation of their cohomology by the Dolbeault complex of forms of moderate growth (see (1.3.8); also cf. $\S 2$ below). The first isomorphism in (1.4.4.1) is a slight generalization of Proposition 3.13.4(i) of [HZ1], and is proved by the same argument. The second isomorphism, which incorporates the rationality assertion, is proved by the argument used to prove Lemma 4.7.14 of [HZ1].

Likewise, the subcanonical extension $[\mathcal{V}]^{\text {sub }}$ is described by conditions of rapid decrease at the boundary. The proof of Proposition 3.13.4(i) of [HZ1] works just as well for rapidly decreasing sections, yielding the first isomorphism of (1.4.4.2). The second isomorphism then follows from the second isomorphism of (1.4.4.1).

Corollary 1.4.5. - Write $\pi_{\Sigma}=\pi_{1, \Sigma} \circ \pi_{2, \Sigma}$. There are spectral sequences:

$$
\begin{aligned}
E_{2}^{p, q}=H^{p}\left({ }_{K_{f}^{1}}^{1} \operatorname{Sh}(G, W \backslash X)_{\Sigma_{1}},\right. & \left.\left(R^{q} \pi_{\Sigma, *}[\mathcal{W}]\right)^{\mathrm{can}}\right) \\
& \Longrightarrow H^{p+q}\left(K_{f}^{2} \operatorname{Sh}(Q / U, U \backslash X)_{\Sigma_{2}},\left[\mathcal{W}_{U}\right]^{\text {can }}\right) \\
& \stackrel{\sim}{\longrightarrow} H^{p+q}\left(K_{f} \operatorname{Sh}(Q, X)_{\Sigma},[\mathcal{W}]^{\text {can }}\right) ; \\
E_{2}^{p, q}=H^{p}\left({ }_{K_{f}^{1}} \operatorname{Sh}(G, W \backslash X)_{\Sigma_{1}},\right. & \left.\left(R^{q} \pi_{\Sigma, *}[\mathcal{W}]\right)^{\mathrm{sub}}\right) \\
& \Longrightarrow H^{p+q}\left(K_{f}^{2} \operatorname{Sh}(Q / U, U \backslash X)_{\Sigma_{2}},\left[\mathcal{W}_{U}\right]^{\text {sub }}\right) \\
& \stackrel{\sim}{\longrightarrow} H^{p+q}\left(K_{f} \operatorname{Sh}(Q, X)_{\Sigma},[\mathcal{W}]^{\text {sub }}\right) .
\end{aligned}
$$

Suppose $[\mathcal{W}]$ satisfies a Kostant condition (see (1.2.16)). Then these spectral sequences degenerate at $E_{2}$ and the filtration induced by the $E_{2}$ term splits canonically.

Proof. - Given Propositions 1.4.3 and 1.4.4, the first part is just the Leray spectral sequence for $\pi_{\Sigma}$. When $[\mathcal{W}]$ satisfies a Kostant condition, the reason for degeneration at $E_{2}$ and the splitting are given in (1.2.16.3). To elaborate further, the prototype of the use of the Kostant condition is given in [HZ1,Corollary 3.7.8], the version of the present corollary for unextended automorphic vector bundles. The degeneration of the spectral sequences for canonical and subcanonical extensions can be found in [HZ1, 3.13-3.14]. These results were obtained by following the proof of [HZ1, Corollary 3.7.8], paying close attention to growth and decay conditions. Proposition 1.4.4 ensures that exactly the same arguments work here. The importance of (1.2.16.2) in proving the splitting was noted in Remark (1.2.16.3).

1.4.6. Periods and rationality. - Mimicking sections 4.7 and 4.8 of [HZ1], we show that the isomorphisms of Propositions (1.4.3) and (1.4.4) are compatible with the canonical rational structures described in (1.2.A). Let $\pi: X \rightarrow W \backslash X$ be the natural map. For $j \geqslant 0$ we define the homogeneous vector bundle $\mathcal{W}_{U^{\prime}}^{(j)}$ on $W \backslash X$ as in the previous section; its fiber at the point $\pi(x) \in W \backslash X$, for $x \in \mathcal{X}$, is given by

$$
\mathcal{W}_{x}^{(j)} \stackrel{\text { def }}{=} H^{j}\left(\mathfrak{v}_{x}^{-}, \mathcal{W}_{x}\right)
$$


Let $\sigma_{x, \mathcal{W}}^{j}$ denote the representation of $\operatorname{Stab}_{W \backslash Q}(\pi(x))$ on $\mathcal{W}_{x}^{(j)}$. The following lemma is the analogue of Lemma 4.7.14 of [HZ1], and is proved in the same way:

Lemma 1.4.6.2. - Let $(T, h) \hookrightarrow(Q, X)$ be a CM pair, as above. Let $L \supset E(T, h ; \mathcal{W})$ and $t_{f} \in T\left(\boldsymbol{A}_{f}\right)$. Then we have

$$
p\left(h, \sigma_{x, \mathcal{W}}^{j}\right) \cdot \operatorname{Per}_{\left(h, t_{f}\right)} \mathcal{W}_{x}^{(j)}(L)=\left.R^{j} \pi_{*}[\mathcal{W}](L)_{\left[h, t_{f}\right]} \subset R^{j} \pi_{*}[\mathcal{W}]\right|_{\left[h, t_{f}\right]}(\mathbb{C}) .
$$

\subsection{A boundary calculation}

In the present section, we generalize Proposition 1.4.5 to a situation arising from the study of the boundary cohomology of pure Shimura varieties. The length of the calculation is primarily due to the complexity of the requisite notation.

Thus, let $(G, X)$ be a pure Shimura datum, which we may as well assume to be standard (i.e., the map $h$ of (1.1.2) is a bijection). For simplicity, we assume $G^{\text {ad }}$ to be $\mathbb{Q}$-simple. Then the standard maximal $\mathbb{Q}$-parabolics of $G$ are totally ordered as in $(0.7)$.

Let $P, P^{\prime}$ be two standard maximal $\mathbb{Q}$-parabolics, with $P^{\prime}<P$. Let $P\left(P^{\prime}\right)=P^{\prime} \cap$ $G_{h, P}$; then $P\left(P^{\prime}\right)$ is a maximal proper parabolic subgroup of $G_{h, P}$, hence corresponds to a boundary stratum in the toroidal compactification of $\operatorname{Sh}\left(G_{h, P}, X(P)\right)$, where $X(P)$ is as in (0.5). Let $Q(P) \subset P$ denote the canonical subgroup, in the terminology of $[\mathrm{P}, \S 4]$ : if $L(P)=G_{h, P} \cdot G_{\ell, P}$ is viewed as a (standard) Levi subgroup of $P$, then $Q(P)=R_{u} P \cdot G_{h, P}$, with associated mixed Shimura datum $(Q(P), \mathcal{X}(P))$. We define the mixed Shimura data $\left(Q\left(P^{\prime}\right), \mathcal{X}\left(P^{\prime}\right)\right)$ and $\left(Q\left(P\left(P^{\prime}\right)\right), \mathcal{X}\left(P\left(P^{\prime}\right)\right)\right)$ analogously. We write

and let

$$
\begin{array}{lll}
Q^{\prime}=Q\left(P^{\prime}\right), & Q=Q(P), & Q^{\prime \prime}=Q\left(P\left(P^{\prime}\right)\right) ; \\
X^{\prime}=X\left(P^{\prime}\right), & X=X(P), & X^{\prime \prime}=X\left(P\left(P^{\prime}\right)\right)=W \backslash X^{\prime},
\end{array}
$$

$$
\begin{array}{cl}
U=U_{Q}=W_{-2} Q, & U^{\prime}=U_{Q^{\prime}}=W_{-2} P^{\prime} \\
W=W_{Q}=R_{u} Q\left(=R_{u} P\right), & W^{\prime}=W_{Q^{\prime}}=\left(W \cap W^{\prime}\right) \cdot U^{\prime} .
\end{array}
$$

Note that $G_{h, P\left(P^{\prime}\right)}=G_{h, P^{\prime}}$ and $U \subset U^{\prime}$.

Let $P_{1}=P\left(P^{\prime}\right) \cdot W$ denote the admissible parabolic of $Q$ corresponding to the parabolic subgroup $P\left(P^{\prime}\right) \subset G_{h, P}$. Then $Q^{\prime} \subset P_{1}$; indeed, $Q^{\prime}$ is precisely the canonical subgroup of $P_{1}$ (cf. [P], Lemma 4.19; note that our $P$ 's are Pink's $Q$ 's and vice versa). In particular, $W \cap U^{\prime} \subset W^{\prime}, U \subset U^{\prime} \cap W, Q^{\prime \prime}=Q^{\prime} /\left(W \cap W^{\prime}\right)$. Let

$$
W^{\prime \prime}=W_{Q^{\prime \prime}}=W^{\prime} /\left(W \cap W^{\prime}\right), \quad U^{\prime \prime}=U_{Q^{\prime \prime}}=U^{\prime} /\left(U^{\prime} \cap W\right) .
$$

Let $V=W / U, V^{\prime}=W^{\prime} / U^{\prime}$, and define $\mathfrak{v}_{x}^{-}=F_{x}^{0}(\operatorname{Lie} Q) \cap \mathfrak{v}, \mathfrak{v}_{x}^{\prime,-}=F_{x}^{0}(\operatorname{Lie} Q) \cap \mathfrak{v}^{\prime}$ as in the paragraph preceding Proposition 1.4.4. Let $V_{x}^{-} \subset V$ and $V_{x}^{\prime,-} \subset V^{\prime}$ be the corresponding subgroups. Let $Q_{P, x}$ (resp. $\left.Q_{P^{\prime}, x}\right)$ be the maximal parabolic subgroup of $K_{x}$ (denoted $Q_{F, x}$ in [HZ1, Lemma 1.8.6]) with unipotent radical $V_{x}^{-}$(resp. $V_{x}^{\prime,-}$ ). Let $K_{h}=K_{x} \cap G_{h, P}, K_{h}^{\prime}=K_{x} \cap G_{h, P^{\prime}}$ (cf. [HZ1, 1.8]). 
The adjoint representation of $G_{h, P}$ on $\mathfrak{v}$ endows the latter with a variation of Hodge structure of weight -1 over $X(P)$, corresponding to the homology in degree one of the abelian scheme $\operatorname{Sh}(Q / U, U \backslash X)$ over $\operatorname{Sh}\left(G_{h, P}, X(P)\right)$. (This is the abelian scheme denoted $\mathcal{A}_{P}$ in [HZ2]; cf. (2.5), below). The maximal parabolic subgroup $P\left(P^{\prime}\right) \subset G_{h, P}$ corresponds to a degeneration to a mixed Hodge structure, with weight filtration denoted $W_{\bullet}^{P\left(P^{\prime}\right)} \mathfrak{v}$, (denoted $W_{\bullet}^{F} \mathfrak{v}$ in $\left.[\mathrm{HZ1}, 1.2 .2]\right)$. We write $\operatorname{Gr}_{i}^{P\left(P^{\prime}\right)} \mathfrak{v}=$ $W_{i}^{P\left(P^{\prime}\right)} V / W_{i-1}^{P\left(P^{\prime}\right)} V$. Let $\mathfrak{v}_{x}^{\prime \prime,-}=F_{x}^{0}(\operatorname{Lie} Q) \cap \operatorname{Gr}_{-1}^{P\left(P^{\prime}\right)} \mathfrak{v}$.

Let $[\mathcal{W}]$ denote a fully decomposed automorphic vector bundle on the original pure Shimura variety $\operatorname{Sh}(G, X)$. Thus for any point $x \in X$, with stabilizer $K_{x} \subset G$, [W] is the locally homogenous holomorphic vector bundle associated to some algebraic representation $\lambda_{x}: K_{x} \rightarrow G L\left(\mathcal{W}_{x}\right)$. In the calculation in [HZ1] of the part of the boundary cohomology of $[\mathcal{W}]$ corresponding to $P$, we associated to $[\mathcal{W}]$ an automorphic vector bundle $[\mathcal{W}(P)]$ (which would be $[\mathcal{W}(F)]$ in the notation of $[\mathrm{HZ1}, 4.7]$ ) on $\operatorname{Sh}(Q, X)$. Concretely, $[\mathcal{W}(P)]$ is the locally homogenous holomorphic vector bundle associated to $\left.\lambda_{x}\right|_{K_{h, P} \cdot V_{x}^{-}}$. Note that $Q_{P, x}$ maps isomorphically to its image in $Q / U$, and the representation $\lambda_{x}$ defines the bundle $\left[\mathcal{W}(P)_{U}\right]($ cf. $(1.2 .14))$ on the abelian scheme $\operatorname{Sh}(Q / U, U \backslash \mathcal{X})$.

Let $\operatorname{Sh}(Q, X) \hookrightarrow \operatorname{Sh}(Q, X)_{\Sigma}$ be a $(\mathrm{SNC})$ toroidal compactification. We will be studying the coherent cohomology of the pullback of the canonical extension $[\mathcal{W}(P)]^{\text {can }}$ to the part of the boundary of $\operatorname{Sh}(Q, X)_{\Sigma}$ corresponding to the admissible parabolic $P_{1}$. Let $D_{\ell, Q^{\prime}}$ denote the quotient $P_{1} / Q^{\prime}$. This is the group that plays the role of $G_{\ell, P^{\prime}}$ in Pink's theory of the toroidal compactification of $\operatorname{Sh}(Q, X)$ (and if $Q$ were reductive, it

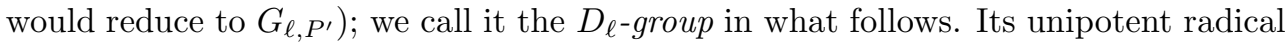
$R_{u} D_{\ell, Q^{\prime}}$ equals $W /\left(W \cap W^{\prime}\right)$, with Lie algebra $\operatorname{Gr}_{0}^{P\left(P^{\prime}\right)} \mathfrak{v}$.

Let $\left(P \cap P^{\prime}\right)_{\ell, P^{\prime}}=P \cap G_{\ell, P^{\prime}}$, a maximal parabolic subgroup of $G_{\ell, P^{\prime}}$ (this is consistent with the notation to be used in $\S 3$ ). Then it is easy to check that

$$
R_{u} D_{\ell, Q^{\prime}}=R_{u}\left(P \cap P^{\prime}\right)_{\ell, P^{\prime}} .
$$

Moreover, the standard Levi subgroup of $\left(P \cap P^{\prime}\right)_{\ell, P^{\prime}}$ is naturally identified with $G_{\ell, P} \cdot G_{\ell, P\left(P^{\prime}\right)}$. Let $P_{1, \ell}=G_{\ell, P\left(P^{\prime}\right)} \cdot R_{u}\left(P \cap P^{\prime}\right)_{\ell, P^{\prime}}$. Then $P_{1}$ is the subgroup $P_{1, \ell} \cdot G_{h, P^{\prime}} \cdot W^{\prime} \subset P$, and there are natural isomorphisms

(1.5.2) $D_{\ell, Q^{\prime}} \stackrel{\sim}{\longrightarrow} P_{1, \ell} /\left(G_{h, P^{\prime}} \cap P_{1, \ell}\right) \stackrel{\sim}{\longrightarrow} G_{\ell, P\left(P^{\prime}\right)} /\left(G_{\ell, P\left(P^{\prime}\right)} \cap G_{h, P^{\prime}}\right) \cdot R_{u}\left(P \cap P^{\prime}\right)_{\ell, P^{\prime}}$.

Note that

$$
\operatorname{Lie}\left(Q_{P, x} \cap Q^{\prime}\right)=\operatorname{Lie}\left(K_{h}^{\prime}\right) \oplus \mathfrak{v}_{x}^{\prime \prime,-} ; \quad \operatorname{Lie}\left(Q_{P^{\prime}, x} \cap Q^{\prime}\right)=\operatorname{Lie}\left(K_{h}^{\prime}\right) \oplus \mathfrak{v}_{x}^{\prime,-} .
$$

In particular, $Q_{P^{\prime}, x} \cap Q^{\prime}$ contains the unipotent radical of $Q_{P^{\prime}, x}$. On the other hand, it follows easily from (1.5.1) that Cayley transform defines an isomorphism

$$
\mathfrak{v}_{x}^{-}=\text {Lie } R_{u} Q_{P, x} \stackrel{\sim}{\longrightarrow} \text { Lie } R_{u} D_{\ell, Q^{\prime}} \oplus \mathfrak{v}_{x}^{\prime \prime,-} .
$$

The choice of a base point $x$ is made only for convenience in characterizing automorphic vector bundles, and plays no essential role in the theory. In what follows, 
the subscript $x$ will be used uniformly to designate a point of any of the homogeneous spaces $X, X^{\prime}, X^{\prime \prime}$, etc., the sole condition being that the various $x$ 's be chosen compatibly with respect to morphisms of mixed Shimura data.

In addition to $(Q / U, U \backslash X)$, and $\left(G_{h, P}, X(P)\right)$, we will be working with the intermediate Shimura data $\left(Q^{\prime} / U, U \backslash X^{\prime}\right)$ and $\left(Q^{\prime} / W, W \backslash X^{\prime}\right)=\left(Q^{\prime \prime}, X^{\prime \prime}\right)$. There are three more relevant Shimura data: $\left(Q^{\prime} / U^{\prime}, U^{\prime} \backslash X^{\prime}\right),\left(Q^{\prime \prime} / U^{\prime \prime}, U^{\prime \prime} \backslash X^{\prime \prime}\right)$, and $\left(Q^{\prime} / W^{\prime}, W^{\prime} \backslash X^{\prime}\right)=\left(G_{h, P^{\prime}}, X\left(P^{\prime}\right)\right)$. We write . where the level subgroups would be indicated. We find three sequences of morphisms

$$
\begin{gathered}
. \operatorname{Sh}\left(Q^{\prime}, X^{\prime}\right) \stackrel{\pi_{2}^{\prime}}{\longrightarrow} \cdot \operatorname{Sh}\left(Q^{\prime} / U^{\prime}, U^{\prime} \backslash X^{\prime}\right) \stackrel{\pi_{1}^{\prime}}{\longrightarrow} \cdot \operatorname{Sh}\left(G_{h, P^{\prime}}, X\left(P^{\prime}\right)\right) \\
. \operatorname{Sh}\left(Q^{\prime \prime}, X^{\prime \prime}\right) \stackrel{\pi_{2}^{\prime \prime}}{\longrightarrow} \cdot \operatorname{Sh}\left(Q^{\prime \prime} / U^{\prime \prime}, U^{\prime \prime} \backslash X^{\prime \prime}\right) \stackrel{\pi_{1}^{\prime \prime}}{\longrightarrow} \cdot \operatorname{Sh}\left(G_{h, P^{\prime}}, X\left(P^{\prime}\right)\right) \\
. \operatorname{Sh}\left(Q^{\prime}, X^{\prime}\right) \stackrel{\pi\left(P^{\prime}\right)_{2}}{\longrightarrow} \cdot \operatorname{Sh}\left(Q^{\prime} / U, U \backslash X^{\prime}\right) \stackrel{\pi\left(P^{\prime}\right)_{1}}{\longrightarrow} \cdot \operatorname{Sh}\left(Q^{\prime \prime}, X^{\prime \prime}\right)
\end{gathered}
$$

The first two are instances of boundary diagram (1.1.5), whereas (1.5.7) is the boundary diagram for the mixed Shimura variety $\operatorname{Sh}(Q, X)$ corresponding to the admissible parabolic subgroup $P_{1} \subset Q$.

We choose compatible families of fans $\Sigma^{\prime}, \Sigma_{2}^{\prime}, \Sigma_{1}^{\prime}$ (resp. $\Sigma^{\prime \prime}, \Sigma_{2}^{\prime \prime}, \Sigma_{1}^{\prime \prime}=\Sigma_{1}^{\prime}$, resp. $\left.\Sigma^{\prime}, \Sigma_{2}, \Sigma_{1}\right)$ so that the morphisms in diagram (1.5.5) (resp. (1.5.6), resp. (1.5.7)) extend to morphisms of toroidal compactifications. By definition we have $\Sigma=\cup\left\{\Sigma_{P_{\alpha}}\right\}$ where $P_{\alpha}$ runs through the standard admissible parabolic subgroups of $Q$, and likewise for the other data. However, to avoid irrelevant notation we designate the toroidal compactification of any of the Shimura varieties ? simply by $?_{\Sigma}$. Again, note that the data for (1.5.7) represent part of the data for the toroidal compactification of $\operatorname{Sh}(Q, X)$. In the end, we obtain a large commutative diagram

$$
\begin{aligned}
& \text {. } \operatorname{Sh}\left(Q^{\prime}, X^{\prime}\right)_{\Sigma} \\
& \pi\left(P^{\prime}\right)_{2, \Sigma} \downarrow \\
& \text {. } \mathrm{Sh}\left(Q^{\prime} / U, U \backslash X^{\prime}\right)_{\Sigma} \\
& \psi_{1} \downarrow \\
& \text {. } \operatorname{Sh}\left(Q^{\prime} / U^{\prime} \cap W,\left(U^{\prime} \cap W\right) \backslash X^{\prime}\right)_{\Sigma} \stackrel{\psi_{2}}{\longrightarrow} . \operatorname{Sh}\left(Q^{\prime} / U^{\prime}, U^{\prime} \backslash X^{\prime}\right)_{\Sigma} \\
& \text {. } \operatorname{Sh}\left(Q^{\prime \prime}, X^{\prime \prime}\right)_{\Sigma} \quad \stackrel{\pi_{2, \Sigma}^{\prime \prime}}{\longrightarrow} . \operatorname{Sh}\left(Q^{\prime \prime} / U^{\prime \prime}, U^{\prime \prime} \backslash X^{\prime \prime}\right)_{\Sigma} \\
& \pi_{1, \Sigma}^{\prime \prime} \downarrow \\
& \text {. } \operatorname{Sh}\left(G_{h, P^{\prime}}, X\left(P^{\prime}\right)\right)_{\Sigma}
\end{aligned}
$$

Here the arrows $\psi_{i}, i=1, \ldots, 4$, have been introduced to make the diagrams commute. We then have

$$
\psi_{2} \circ \psi_{1} \circ \pi\left(P^{\prime}\right)_{2, \Sigma}=\pi_{2, \Sigma}^{\prime} ; \quad \pi_{1, \Sigma}^{\prime \prime} \circ \psi_{4}=\pi_{1, \Sigma}^{\prime} ; \psi_{3} \circ \psi_{1}=\pi\left(P^{\prime}\right)_{1, \Sigma}
$$


One checks that $\psi_{1}$ and $\psi_{2}$ are compactifications of toric fibrations, whereas $\psi_{3}$ and $\psi_{4}$ are generically abelian schemes. The left-hand column in (1.5.8), a refinement of (1.5.7), will be analyzed below in connection with the $Q^{\prime}$-part of the boundary in the compactification of the diagram (1.1.5):

$$
\text { • } \operatorname{Sh}(Q, X)_{\Sigma} \stackrel{\pi_{2}}{\longrightarrow} \cdot \operatorname{Sh}(Q / U, U \backslash \mathcal{X})_{\Sigma} \stackrel{\pi_{1}}{\longrightarrow} \cdot \operatorname{Sh}\left(G_{h, P}, W \backslash X\right)_{\Sigma} .
$$

At no point in our calculation of boundary coherent cohomology have we seriously used the fact that $W$ is the full unipotent radical of $Q$. The only real restriction is the Kostant condition, used to prove splitting in Proposition 1.4.5. We now apply the analysis of $[\mathrm{HZ} 1, \S 3]$ to study the boundary cohomology of $[\mathcal{W}(P)]$. We begin by choosing a connected component $D \subset X$ and replacing the disconnected (finite-level) Shimura varieties $\bullet \operatorname{Sh}(Q, \mathcal{X})$ by a connected component of the form $M_{\Gamma}=\Gamma \backslash D$. As above, we use the subscript $\Sigma$ uniformly for all (compatible) toroidal compactifications. Recall that $M_{\Gamma}$ has a Baily-Borel compactification $M_{\Gamma}^{*}[\mathrm{P}, 6.2]$, defined settheoretically as the disjoint union of boundary strata $M_{\Gamma}\left(P_{\alpha}\right)$ for $\Gamma$-conjugacy classes of rational parabolic subgroups $P_{\alpha}$ of $Q$. There is a surjective map $\phi_{\Sigma}: M_{\Gamma, \Sigma} \rightarrow M_{\Gamma}^{*}$. We let $Z_{Q^{\prime}} \subset M_{\Gamma, \Sigma}$ denote the closure of the subset $\phi_{\Sigma}^{-1} M_{\Gamma}\left(P_{1}\right)$ (see [HZ1,(1.5.1)] for the analogue in the pure setting). Under our hypotheses, it is a divisor with normal crossings, each of whose components is smooth. We denote by $i_{Q^{\prime}}$ the closed immersion $Z_{Q^{\prime}} \hookrightarrow M_{\Gamma, \Sigma}$.

As in the pure case (cf. [HZ1,1.3]), the fan $\Sigma_{P_{1}}$ is a polyhedral decomposition of a certain subset of the closure of a cone $C\left(Q^{\prime}\right) \subset U^{\prime}(\mathbb{R})(-1)$ (the Tate twist is as in $[\mathrm{P}])$, homogeneous with respect to $P_{1}(\mathbb{R})^{0}$ and invariant under translation by $\left(U^{\prime} \cap W\right)(\mathbb{R})(-1)$. Pink proves that the quotient $C\left(Q^{\prime}\right) /\left(U^{\prime} \cap W\right)(\mathbb{R})(-1)$ is a non-degenerate homogeneous cone for $G_{\ell, P\left(P^{\prime}\right)}(\mathbb{R})^{0}$ in $U_{P\left(P^{\prime}\right)}(\mathbb{R})(-1)$ (for all this, see $[\mathrm{P}]$, Proposition 4.15). The fan $\Sigma_{P_{1}}$ is equivariant with respect to an appropriate congruence subgroup $\Delta_{\ell, Q^{\prime}} \subset D_{\ell, Q^{\prime}}(\mathbb{Q})$ (denoted $\Delta_{1}$ by Pink in its adelic incarnation). Our running hypothesis that $\Gamma$ be neat (cf. (1.3)) implies that $\Delta_{\ell, Q^{\prime}}$ acts freely on $\Sigma_{P_{1}}$. We may assume without loss of generality that $\Delta_{\ell, Q^{\prime}}$ admits a factorization as semi-direct product

$$
\Delta_{\ell, Q^{\prime}} \stackrel{\sim}{\longrightarrow} \Gamma_{\ell, P\left(P^{\prime}\right)} \times \Gamma_{u},
$$

where $\Gamma_{\ell, P\left(P^{\prime}\right)}$ is the image in $G_{\ell, P\left(P^{\prime}\right)} /\left(G_{\ell, P\left(P^{\prime}\right)} \cap G_{h, P^{\prime}}\right)(\mathbb{Q})$ of a congruence subgroup $\Gamma^{1}$ of $G_{\ell, P\left(P^{\prime}\right)}(\mathbb{Q})$ and $\Gamma_{u}$ is a lattice in the vector group $R_{u} D_{\ell, Q^{\prime}}$. (The hypothesis that $\Gamma$ be neat implies that the map $\Gamma^{1} \rightarrow \Gamma_{\ell, P\left(P^{\prime}\right)}$ is an isomorphism.)

We define $\Sigma_{P_{1}}^{c}$ and $\widehat{\Sigma}_{P_{1}}^{c}$ as in [HZ1, (1.3.2)] and [HZ1,(2.2.2)], respectively. The irreducible components of $Z_{Q^{\prime}}$ are indexed by the one-dimensional faces in $\Sigma_{P_{1}}$ modulo the action of $\Delta_{\ell, Q^{\prime}}$. Then it is easy to verify that the analogue of Lemma 3.7 .2 of [HZ1] remains true in the present setting:

LemMA 1.5.12. - The nerve $\mathfrak{N}\left(\mathcal{Z}_{Q^{\prime}}\right)$ of the closed covering of $Z_{Q^{\prime}}$ by its irreducible components is isomorphic to $\Delta_{\ell, Q^{\prime}} \backslash \widehat{\Sigma}_{P_{1}}^{c}$. 
As before, we write - where the level subgroups belong, to avoid proliferation of notation. We now consider the restriction of (1.5.10) to the connected component $M_{\bullet, \Sigma}$

$$
M_{\bullet, \Sigma} \stackrel{\pi_{2}}{\longrightarrow} \mathcal{A}_{Q / U, \bullet, \Sigma} \stackrel{\pi_{1}}{\longrightarrow} M_{P, \bullet, \Sigma}
$$

here, $\mathcal{A}_{Q / U, \bullet, \Sigma}\left(\right.$ resp. $\left.M_{P, \bullet, \Sigma}\right)$ is a connected component of a toroidal compactification of $\bullet \operatorname{Sh}(Q / U, U \backslash X)$ (resp. $\left.\bullet \operatorname{Sh}\left(G_{h, P}, X^{\prime \prime}\right)\right)$. It follows from Pink's theory that $\mathcal{A}_{Q^{\prime} / U, \bullet, \Sigma}$ is a toroidal compactification of a semi-abelian scheme over $M_{Q^{\prime \prime}, \bullet, \Sigma}$. There is a commutative diagram:

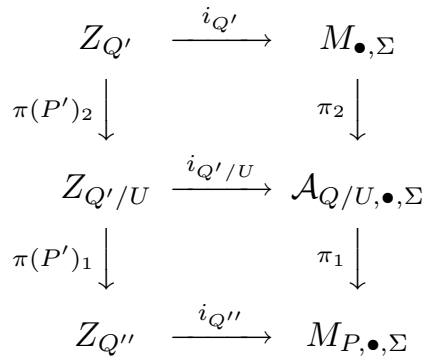

As in the discussion of $\Sigma_{P_{1}}$ above, the second and third lines of (1.5.14) correspond to fans

$\Sigma_{P_{1} / U} \subset C\left(Q^{\prime} / U\right) \subset U^{\prime} / U(\mathbb{R})(-1) \quad$ and $\quad \Sigma_{P_{1} / W} \subset C\left(Q^{\prime \prime}\right) \subset\left(U^{\prime} / U^{\prime} \cap W\right)(\mathbb{R})(-1)$, respectively, with the obvious notation. The corresponding $D_{\ell^{-}}$groups are denoted $D_{\ell, Q^{\prime} / U}$ and $D_{\ell, Q^{\prime \prime}}$. It is easy to see that there are maps

$$
\Sigma_{P_{1}} \longrightarrow \Sigma_{P_{1} / U} \longrightarrow \Sigma_{P_{1} / W},
$$

where the first map is a bijection, and that

$$
\begin{aligned}
D_{\ell, Q^{\prime}} & \stackrel{\sim}{\longrightarrow} D_{\ell, Q^{\prime} / U} ; \quad D_{\ell, Q^{\prime \prime}} \stackrel{\sim}{\longrightarrow} D_{\ell, Q^{\prime} / U} / R_{u} D_{\ell, Q^{\prime} / U} \stackrel{\sim}{\longrightarrow} G_{\ell, P\left(P^{\prime}\right)}, \\
C\left(Q^{\prime} / U\right) & \stackrel{\sim}{\longrightarrow} C\left(Q^{\prime}\right) / U(\mathbb{R})(-1) ; \quad C\left(Q^{\prime \prime}\right) \stackrel{\sim}{\longrightarrow} C\left(Q^{\prime}\right) /\left(U^{\prime} \cap W\right)(\mathbb{R})(-1) .
\end{aligned}
$$

The two lines of (1.5.15) are compatible. As an immediate consequence, we have the following elaboration on Lemma (1.5.12):

Lemma 1.5.16. - Define $\mathfrak{N}\left(\mathcal{Z}_{Q^{\prime} / U}\right)$ and $\mathfrak{N}\left(\mathcal{Z}_{Q^{\prime \prime}}\right)$ to be the nerves of the closed coverings of $Z_{Q^{\prime} / U}$ and $Z_{Q^{\prime \prime}}$, respectively, by their irreducible components. Then there is a diagram

$$
\mathfrak{N}\left(\mathcal{Z}_{Q^{\prime}}\right) \stackrel{\mathfrak{N}\left(\pi\left(P^{\prime}\right)_{2}\right)}{\longrightarrow} \mathfrak{N}\left(\mathcal{Z}_{Q^{\prime} / U}\right) \stackrel{\mathfrak{N}\left(\pi\left(P^{\prime}\right)_{1}\right)}{\longrightarrow} \mathfrak{N}\left(\mathcal{Z}_{Q^{\prime \prime}}\right)
$$

where the first arrow is an isomorphism and the second is homotopy equivalent to a fibration with fiber $\Gamma_{u} \backslash\left[\left(U^{\prime} \cap W / U\right)(\mathbb{R})(-1)\right]$.

To each $\sigma \in \Sigma_{P_{1}}$ corresponds an irreducible closed stratum $Z_{\sigma} \subset Z_{Q^{\prime}}$, a toroidal compactification of a connected component of the mixed Shimura variety. $\operatorname{Sh}\left(Q^{\prime} / U(\sigma), X^{\prime}\right)$, where $U(\sigma) \subset U^{\prime}$ is the rational subgroup whose group of real 
points is generated by $\sigma$. The left-hand column of (1.5.14), restricted to $Z_{\sigma}$, is a diagram of connected components for the analogue of (1.5.7) where $Q^{\prime}$ is replaced by $Q^{\prime} / U(\sigma)$. Fix any such $Z_{\sigma}$. Then the left hand column of (1.5.8) gives rise to a diagram of connected components:

$$
Z_{\sigma} \stackrel{\pi\left(P^{\prime}\right)_{2}}{\longrightarrow} \mathcal{A}_{Q^{\prime} /(U+U(\sigma)), \bullet, \Sigma} \stackrel{\psi_{1}}{\longrightarrow} \mathcal{A}_{Q^{\prime} /\left(U^{\prime} \cap W+U(\sigma)\right), \bullet, \Sigma} \stackrel{\psi_{3}}{\longrightarrow} Z_{\sigma^{\prime \prime}}
$$

Here $\sigma^{\prime \prime}$ denotes the image of $\sigma$ in $C\left(Q^{\prime \prime}\right)$, and $Z_{\sigma^{\prime \prime}}=M_{Q^{\prime \prime} / U\left(\sigma^{\prime \prime}\right), \bullet, \Sigma}$ is the corresponding closed stratum in $Z_{Q^{\prime \prime}}$. As $\sigma^{\prime \prime}$ varies, the diagrams (1.5.17) fit together to a refinement of the left-hand column of (1.5.14):

$$
Z_{Q^{\prime}} \stackrel{\pi\left(P^{\prime}\right)_{2}}{\longrightarrow} Z_{Q^{\prime} / U} \stackrel{\psi_{1}}{\longrightarrow} \mathcal{A}_{Q^{\prime} / U^{\prime} \cap W} \stackrel{\psi_{3}}{\longrightarrow} Z_{Q^{\prime \prime}}
$$

This can be continued with a map $\pi^{\prime \prime}: Z_{Q^{\prime \prime}} \rightarrow M_{P^{\prime}, \bullet, \Sigma}$, where $M_{P^{\prime}, \bullet, \Sigma}$ is a connected component of $\operatorname{Sh}\left(G_{h, P^{\prime}}, X\left(P^{\prime}\right)\right)_{\Sigma}$, that is the toroidal compactification of (1.5.6).

Fix $\sigma^{\prime \prime} \subset C\left(Q^{\prime \prime}\right)$, and let $Z_{Q^{\prime}}\left(\sigma^{\prime \prime}\right) \subset Z_{Q^{\prime}}$ denote the union of the strata $Z_{\sigma}$ that map to $Z_{\sigma^{\prime \prime}}$ under $\pi\left(P^{\prime}\right):=\pi\left(P^{\prime}\right)_{1} \circ \pi\left(P^{\prime}\right)_{2}$. Lemma 1.5.16 and the discussion below diagram (1.5.8) imply that

(1.5.19) The nerve $\mathfrak{N}\left(Z_{Q^{\prime}}\left(\sigma^{\prime \prime}\right)\right)$ of the closed covering of $Z_{Q^{\prime}}\left(\sigma^{\prime \prime}\right)$ by its irreducible components is homotopy equivalent to the compact torus $\Gamma_{u} \backslash\left[\left(U^{\prime} \cap W / U\right)(\mathbb{R})(-1)\right]$ (more precisely, it is isomorphic to a triangulation of the latter).

(1.5.20) Over $\pi^{\prime \prime,-1}\left(M_{P^{\prime}, \bullet}\right)$ (i.e., over the open stratum), $\psi_{3}$ is an abelian scheme whose typical anti-holomorphic tangent space is $\mathfrak{v}_{x}^{\prime \prime,-}$; in particular, it is a bijection on sets of irreducible components.

(1.5.21) $\pi\left(P^{\prime}\right)_{2}$ is a bijection on sets of irreducible components.

By Proposition (1.4.3), we have canonical isomorphisms

$$
\begin{aligned}
& \pi\left(P^{\prime}\right)_{2, *}\left([\mathcal{W}(P)]^{\text {can }}\right) \stackrel{\sim}{\longrightarrow} i_{Q^{\prime} / U}^{*}\left[\mathcal{W}(P)_{U}\right]^{\text {can }} ; \\
& R^{i} \pi\left(P^{\prime}\right)_{2, *}\left([\mathcal{W}(P)]^{\text {can }}\right)=0, \quad i>0 .
\end{aligned}
$$

Similarly, there is an automorphic vector bundle that we might denote $\left[\mathcal{W}\left(P^{\prime}\right)_{U^{\prime} \cap W}\right]$ on $\operatorname{Sh}\left(Q^{\prime} /\left(U^{\prime} \cap W\right),\left(U^{\prime} \cap W\right) \backslash X^{\prime \prime}\right)$ such that, for any diagram of closed strata as in (1.5.17), the pullback to $\mathcal{A}_{Q^{\prime} /(U+U(\sigma)), \bullet, \Sigma}$ of $i_{Q^{\prime} / U}^{*}\left[\mathcal{W}(P)_{U}\right]^{\text {can }}$ is isomorphic to $\psi_{1}^{*}\left[\mathcal{W}\left(P^{\prime}\right)_{U^{\prime} \cap W}\right]$.

It then follows from (1.5.19) and (1.5.20), as in [HZ1, (3.12.6)], that

$$
R^{q} \pi\left(P^{\prime}\right)_{*}\left(i_{Q^{\prime}}^{*}[\mathcal{W}(P)]^{\text {can }}\right) \simeq \mathcal{H}^{q}\left(R_{\Gamma_{u}} \psi_{3, *}\left[\mathcal{W}(P)_{U^{\prime} \cap W}\right]^{\text {can }}\right) ;
$$

here recall (cf. (1.5.9)) that $\pi\left(P^{\prime}\right)$ is the composite of the three arrows in (1.5.18). The notation $R_{\Gamma_{u}} \psi_{3, *}$ for equivariant higher direct images is as in [HZ1], e.g. [HZ1,3.12.4]. Proposition 1.5.24. - The Leray spectral sequence

$$
E_{2}^{p, q}=H^{p}\left(Z_{Q^{\prime \prime}}, R^{q} \pi\left(P^{\prime}\right)_{*}\left(i_{Q^{\prime}}^{*}[\mathcal{W}(P)]^{\text {can }}\right)\right) \Longrightarrow H^{p+q}\left(Z_{Q^{\prime}}, i_{Q^{\prime}}^{*}[\mathcal{W}(P)]^{\text {can }}\right)
$$

degenerates at $E_{2}$ and the gradation induced by the $E_{2}$ term splits canonically. 
Proof. - As in the proof of Corollary 1.4.5, the point is to apply an appropriate Kostant condition, in this case to the mixed Shimura datum $\left(Q^{\prime} / U, U \backslash X^{\prime}\right)$. The group $K_{x} \subset G$ stabilizing $x \in X$ is the $K_{x}$ of (1.2.16.1); for $\Pi_{x}$ we take $Q_{P, x}$. Instead of the full subgroup $Q_{x}^{0} \cap\left(W^{\prime} / U\right)$ for the mixed Shimura datum $\left(Q^{\prime} / U, U \backslash X^{\prime}\right)$, we take the subgroup $Q_{x}^{0} \cap(W / U)=Q_{x}^{0} \cap V$, whose Lie algebra is just $\mathfrak{v}_{x}^{-}$. The map

$$
\alpha_{x}: \mathfrak{v}_{x}^{-} \stackrel{\sim}{\longrightarrow} \operatorname{Lie}\left(R_{u} Q_{P, x}\right)
$$

is defined, as in $[H Z 1,1.8 .6]$, in terms of the Cayley transform for the boundary subgroup $P$ of $G$. Thus we are in the situation of (1.2.16.4). and we use the canonical splitting (1.2.16.5). Note that all choices are canonical with respect to our choice of the point $x$, the standard parabolics $P, P^{\prime}$, and the standard Levi components. In this sense, the splitting we will obtain on the $E_{2}$ term is also canonical.

We next set up the Leray spectral sequence, so that we may apply the above. The automorphic vector bundle $\left[\mathcal{W}(P)_{U^{\prime} \cap W}\right]$ is the locally homogeneous holomorphic vector bundle associated to the restriction to

$$
Q_{P, x} \cap Q^{\prime} \simeq\left(Q_{P, x} \cap Q^{\prime}\right) /\left(Q_{P, x} \cap Q^{\prime} \cap U^{\prime} \cap W\right)
$$

of the representation $\lambda_{x}: K_{x} \rightarrow G L\left(\mathcal{W}_{x}\right)$ introduced near the beginning of (1.5). In particular, its fiber at $x$ is $\mathcal{W}_{x}$. Just as in Proposition 1.4.4,

$$
R^{q} \psi_{3, *}\left[\mathcal{W}(P)_{U^{\prime} \cap W}\right]^{\text {can }} \stackrel{\sim}{\longrightarrow}\left[\mathcal{W}(P)^{(q),{ }^{\prime \prime}}\right]^{\text {can }}
$$

where $\left[\mathcal{W}(P)^{\left.(q),{ }^{\prime \prime}\right]}\right.$ is the automorphic vector bundle whose fiber at $x$ is

$$
H^{q}\left(\operatorname{Lie} R_{u}\left(Q_{P, x} \cap Q^{\prime}\right), \mathcal{W}_{x}\right)=H^{q}\left(\mathfrak{v}_{x}^{\prime \prime,-}, \mathcal{W}_{x}\right) ;
$$

the equality follows from (1.5.3).

Next, $\Gamma_{u}$ acts trivially on $\mathcal{A}_{Q^{\prime} / U^{\prime} \cap W}$, hence the $\Gamma_{u}$-equivariant cohomology on the right-hand side of (1.5.23) is computed fiber by fiber. Thus $\mathcal{H}^{q}\left(R_{\Gamma_{u}} \psi_{3, *}\left[\mathcal{W}(P)_{U^{\prime} \cap W}\right]^{\text {can }}\right)$ is the automorphic vector bundle whose fiber at $x$ is

$$
\mathbb{H}^{q}\left(R\left(\Gamma_{u}\right) \circ R\left(\mathfrak{v}_{x}^{\prime \prime,-}\right)\left(\mathcal{W}_{x}\right)\right) .
$$

Here $R\left(\Gamma_{u}\right)$ and $R\left(\mathfrak{v}_{x}^{\prime \prime,-}\right)$ are abbreviations for the right-derived functors of $H^{0}\left(\Gamma_{u}, \bullet\right)$ and $H^{0}\left(\mathfrak{v}_{x}^{\prime \prime,-}, \bullet\right)$, respectively. We use the analogous notation for Lie algebra cohomology below. Now by Nomizu's theorem (cf. [HZ2, (5.4.7)]), there is a canonical isomorphism of functors

$$
R\left(\Gamma_{u}, \bullet\right) \stackrel{\sim}{\longrightarrow} R\left(\operatorname{Lie} R_{u} D_{\ell, Q^{\prime}}, \bullet\right) .
$$

It follows from (1.5.4) that the group in (1.5.24.3) is canonically isomorphic to $H^{q}\left(\mathfrak{v}_{x}^{-}, \mathcal{W}_{x}\right)$. Let $\left[\mathcal{W}\left(Q^{\prime \prime}\right)^{(q)}\right]^{\text {can }}$ be the canonically extended automorphic vector bundle on $Z_{Q^{\prime \prime}}$ associated to the representation of $Q_{P\left(P^{\prime}\right), x}$ on $H^{q}\left(\mathfrak{v}_{x}^{-}, \mathcal{W}_{x}\right)$. We have thus constructed a canonical isomorphism

$$
\mathcal{H}^{q}\left(R_{\Gamma_{u}} \psi_{3, *}\left[\mathcal{W}(P)_{U^{\prime} \cap W}\right]^{\text {can }}\right) \stackrel{\sim}{\longrightarrow}\left[\mathcal{W}\left(Q^{\prime \prime}\right)^{(q)}\right]^{\text {can }}
$$


(this is obvious without the canonical extensions, and that it holds with them follows from using growth conditions, as in the proof of Proposition (1.4.4).)

By (1.5.23), the Leray spectral sequence can thus be rewritten

$$
E_{2}^{p, q}=H^{p}\left(\left(Z_{Q^{\prime \prime}},\left[\mathcal{W}\left(Q^{\prime \prime}\right)^{(q)}\right]^{\text {can }}\right)\right) \Longrightarrow H^{p+q}\left(Z_{Q^{\prime}}, i_{Q^{\prime}}^{*}[\mathcal{W}(P)]^{\text {can }}\right) .
$$

Since $\mathfrak{v}_{x}^{-}$is the unipotent radical of a parabolic subalgebra of $K_{x}$, the Kostant condition is satisfied, and the degeneration and splitting follow (as described at the beginning of the proof).

One verifies from the explicit descriptions in terms of $\lambda_{x}$ that, for each $q$, $\left[\mathcal{W}\left(Q^{\prime \prime}\right)^{(q)}\right]^{\text {can }}$ is the pullback via $i_{Q^{\prime \prime}}$ of the canonically extended fully decomposed automorphic vector bundle $\left[\mathcal{W}(P)^{(q)}\right]^{\text {can }}$ on the (connected) pure Shimura variety $M_{P, \bullet, \Sigma}$; notation is as in Proposition (1.4.4). Applying Kostant's theorem, Proposition (1.5.24) yields an isomorphism:

$$
H^{n}\left(Z_{Q^{\prime}}, i_{Q^{\prime}}^{*}[\mathcal{W}(P)]^{\text {can }}\right) \stackrel{\sim}{\longrightarrow} \bigoplus_{b \in W^{P, x}} H^{n-\ell(b)}\left(Z_{Q^{\prime \prime}}, i_{Q^{\prime \prime}}^{*}\left[\mathcal{V}_{\lambda(h, b)}\right]^{\text {can }} \otimes V_{\lambda(\ell, b)}\right)
$$

as in $[\mathrm{HZ1} 1,(3.7 .4)]$. Thus we let

$$
\left[\mathcal{W}\left(Q^{\prime \prime}\right)_{\lambda(h, b)}\right]^{\text {can }}=i_{Q^{\prime \prime}}^{*}\left[\mathcal{V}_{\lambda(h, b)}\right]^{\text {can }} .
$$

Then we can apply the analysis of $\S 3$ of $[\mathrm{HZ1}]$ to the cohomology of $\left[\mathcal{W}\left(Q^{\prime \prime}\right)_{\lambda(h, b)}\right]$ to obtain

$$
\begin{aligned}
& (1.5 .26) \quad H^{s}\left(Z_{Q^{\prime \prime}},\left[\mathcal{W}\left(Q^{\prime \prime}\right)_{\lambda(h, b)}\right]^{\mathrm{can}}\right) \stackrel{\sim}{\longrightarrow} \\
& \bigoplus_{a \in W^{P\left(P^{\prime}\right), x}} \bigoplus_{r} H^{s-r-\ell(a)}\left(M_{P\left(P^{\prime}\right), \bullet, \Sigma},\left[\mathcal{W}\left(Q^{\prime \prime}\right)_{\lambda(h, b) ; \lambda(h, a)}\right]^{\mathrm{can}}\right) \otimes H^{r}\left(\Gamma_{\ell, P\left(P^{\prime}\right)}, V_{\lambda(h, b) ; \lambda(\ell, a)}\right) .
\end{aligned}
$$

Here the first term $\lambda(h, b)$ in the subscript corresponds to the decomposition (1.5.25), whereas the second term corresponds to the decomposition relative to the parabolic $Q_{P\left(P^{\prime}\right), x} \subset K_{h, P}$. Note that $M_{P\left(P^{\prime}\right), \bullet, \Sigma}$ coincides with $M_{P^{\prime}, \bullet, \Sigma}$, (compare the final terms in (1.5.5) and (1.5.6)).

Let $R=P \cap P^{\prime}$; the standard Levi factor of $R$ is $G_{\ell, R} \times G_{h, P^{\prime}}$, where $G_{\ell, R}$ is the standard Levi factor of $R \cap G_{\ell, P^{\prime}}$. Let $Q^{R}=Q_{P, x} \cap Q_{P^{\prime}, x}$. This is a parabolic subgroup of $K_{x}$ with Levi factor isomorphic via Cayley transform to $G_{\ell, R} \times K_{h, P^{\prime}}$. Let $W^{R}\left(=W^{R, x}\right)$ be the corresponding set of Kostant representatives in the Weyl group of $K_{x}$. Then (see Lemma 3.1.6 below), we can combine the decompositions (1.5.25) and (1.5.26) to write:

$$
\begin{aligned}
H^{n}\left(Z_{Q^{\prime}}, i_{Q^{\prime}}^{*}[\mathcal{W}(P)]^{\text {can }}\right) \stackrel{\sim}{\longrightarrow} \\
\bigoplus_{w \in W^{R}} \bigoplus_{r} H^{n-\ell(w)-r}\left(M_{P^{\prime}, \bullet, \Sigma},\left[\mathcal{V}_{\lambda(h, w)}\right]^{\text {can }}\right) \otimes H^{r}\left(\Gamma_{\ell, P\left(P^{\prime}\right)}, V_{\lambda(\ell, w)}\right) .
\end{aligned}
$$

More general results are obtained in section 3.3. The decomposition (1.5.27) can be compared with (3.3.1), below. Note, however, that the second factor on the right 
here is the cohomology of $\Gamma_{\ell, P\left(P^{\prime}\right)}$ and not that of a congruence subgroup of $G_{\ell, R}(\mathbb{Q})$. Indeed, as in (3.3.4), below, we have $G_{\ell, R}=G_{\ell, P} \cdot G_{\ell, P\left(P^{\prime}\right)}$.

This is the "long way" to calculate the left-hand side of (1.5.27). The short way is to apply Corollary (1.4.5) to the different strata $Z_{\sigma}$ of $Z_{Q^{\prime}}$, and to use the analogue of diagram (1.5.5) for the mixed Shimura varieties. $\mathrm{Sh}\left(Q^{\prime} / U(\sigma), X^{\prime}\right)$ (cf. (1.5.17)) as $\sigma$ varies:

$$
\text { . } \operatorname{Sh}\left(Q^{\prime} / U(\sigma), X^{\prime}\right) \stackrel{\pi_{2}^{\prime}}{\longrightarrow} . \operatorname{Sh}\left(Q^{\prime} / U^{\prime}, U^{\prime} \backslash X^{\prime}\right) \stackrel{\pi_{1}^{\prime}}{\longrightarrow} . \operatorname{Sh}\left(G_{h, P^{\prime}}, X\left(P^{\prime}\right)\right) .
$$

Note that the last two terms of (1.5.28) are independent of $\sigma$. Applying Lemma 1.5.12 directly to the configuration of the $Z_{\sigma}$, we find the following equivariant version of (1.4.5.1):

Lemma 1.5.29. - There is a spectral sequence

$$
E_{2}^{p, q}=H_{\Delta_{\ell, Q^{\prime}}}^{p}\left(M_{P^{\prime}, \bullet, \Sigma}, R^{q} \pi_{*}^{\prime} i_{Q^{\prime}}^{*}[\mathcal{W}(P)]^{\text {can }}\right) \Longrightarrow H^{p+q}\left(Z_{Q^{\prime}}, i_{Q^{\prime}}^{*}[\mathcal{W}(P)]^{\text {can }}\right) .
$$

This spectral sequence degenerates at $E_{2}$ and the filtration induced on the abutment splits canonically.

We are using the fact that $i_{Q^{\prime}}^{*}[\mathcal{W}(P)]^{\text {can }}$ is, on each $Z_{\sigma}$, isomorphic to the restriction to $Z_{\sigma}$ of $\left[\mathcal{W}\left(P^{\prime}\right)\right]^{\text {can }}$. This is because $\bullet \operatorname{Sh}(Q, X)_{\Sigma}$, can be realized as a part of a toroidal compactification of $\bullet \operatorname{Sh}(G, X)$, and then $Z_{Q^{\prime}}$ is part of the $P^{\prime}$-stratum of the latter. Then the lemma follows from the characterization [HZ1, (3.2.2)] of the canonical extension on $\operatorname{Sh}(G, X)_{\Sigma}$. The last assertions are a consequence of the Kostant condition, applied essentially as in (1.5.24) (cf. [HZ1, (3.13.6)]). In this case $\Pi_{x}=Q_{P^{\prime}, x} \subset K_{x}$, and $\operatorname{Lie}\left(Q_{x}^{0}\right)=\mathfrak{v}_{x}^{\prime,-}$, with the isomorphism $\alpha_{x}$ being given by Cayley transform as in [HZ1,(1.8.6)].

We can write the splitting in (1.5.29) in terms of Kostant representatives in $W^{P^{\prime}}$, as above. Since $\Delta_{\ell, Q^{\prime}} \subset G_{\ell, P^{\prime}}$, we obtain an isomorphism

$$
\begin{aligned}
H^{n}\left(Z_{Q^{\prime}}, i_{Q^{\prime}}^{*}\right. & {\left.[\mathcal{W}(P)]^{\text {can }}\right) } \\
& \stackrel{\sim}{\longrightarrow} \bigoplus_{t \in W^{P^{\prime}, x}} H^{n-r-\ell(t)}\left(M_{P^{\prime}, \bullet, \Sigma},\left[\mathcal{V}_{\lambda(h, t)}\right]^{\text {can }}\right) \otimes H^{r}\left(\Delta_{\ell, Q^{\prime}}, V_{\lambda(\ell, t)}\right) .
\end{aligned}
$$

The last factor on the right can be computed by a Hochschild-Serre spectral sequence via (1.5.11). Recalling that $\Gamma_{u}$ is a lattice in the unipotent radical of $R_{\ell, P^{\prime}}:=R \cap G_{\ell, P^{\prime}}$, (see (1.5.1)) and applying Nomizu's theorem and Kostant's theorem again, we have

$$
H^{r}\left(\Delta_{\ell, Q^{\prime}}, V_{\lambda(\ell, t)}\right) \stackrel{\sim}{\longrightarrow} \bigoplus_{s \in W^{R_{\ell, P^{\prime}}}} H^{r-\ell(s)}\left(\Gamma_{\ell, Q^{\prime}}, V_{\lambda(\ell, t) ; \lambda(\ell, s)}\right) .
$$

Applying Lemma 3.1.6 (below) again, we can combine the last two formulas. As a special case of the formulas in Section 3.3 below, we find that, if $w \in W^{R}$ equals st, with $s \in W^{R_{\ell, P^{\prime}}}$ and $t \in W^{P^{\prime}, x}$ then

$$
\begin{aligned}
{\left[\mathcal{V}_{\lambda(h, t)}\right] } & \simeq\left[\mathcal{V}_{\lambda(h, w)}\right] ; \\
V_{\lambda(\ell, t) ; \lambda(\ell, s)} & \simeq V_{\lambda(\ell, w)},
\end{aligned}
$$


where the right-hand sides are as in (1.5.27). Thus (1.5.29) gives another isomorphism

$$
\begin{aligned}
& H^{n}\left(Z_{Q^{\prime}}, i_{Q^{\prime}}^{*}\right.\left.\mathcal{W}(P)]^{\text {can }}\right) \stackrel{\sim}{\longrightarrow} \\
& \bigoplus_{w \in W^{R}} \bigoplus_{r} H^{n-\ell(w)-r}\left(M_{P^{\prime}, \bullet, \Sigma},\left[\mathcal{V}_{\lambda(h, w)}\right]^{\text {can }}\right) \otimes H^{r}\left(\Gamma_{\ell, P\left(P^{\prime}\right)}, V_{\lambda(\ell, w)}\right) .
\end{aligned}
$$

Proposition 1.5.34. - The isomorphisms (1.5.27) and (1.5.33) are the same.

Proof. - This comes down to the commutativity of the diagram (1.5.8). The short calculation goes by way of the maps $\psi_{4}$ and $\psi_{2}$, whereas the long one involves $\pi_{2, \Sigma}^{\prime \prime}$ and $\psi_{3}$ (use (1.5.9) in both cases).

\section{REMARKS 1.5.35}

(i) Proposition 1.5.34 is the point of this section. It is applied at the end of section 3.4. The key step is the proof of Proposition 1.5.24, where the Kostant condition is obtained only thanks to the intervention of the discrete group $\Gamma_{u}$.

(ii) Throughout this section, the Shimura datum $(Q, X)$ can be replaced by $\left(Q / U_{0}, U_{0} \backslash X\right)$ for any rational subgroup $U_{0} \subset U$. In this way, the simplicial arguments of $[\mathrm{HZ1} 1,3.7]$ can be applied to extend the results of this section to the closed stratum $Z_{P}$ of a toroidal compactification of (a connected component of) $\bullet \operatorname{Sh}(G, X)$. This amounts to replacing $\Gamma_{\ell, P\left(P^{\prime}\right)}$ by $\Gamma_{\ell, P} \times \Gamma_{\ell, P\left(P^{\prime}\right)}$ on the right-hand of formulas (1.5.27) and (1.5.33). As noted after (1.5.27), $\Gamma_{\ell, P} \times \Gamma_{\ell, P\left(P^{\prime}\right)}$ is a congruence subgroup of $G_{\ell, R}(\mathbb{Q})$, and the results of this section come down to showing that two ways of calculating the coherent cohomology of $Z_{P}$ give the same result.

(iii) The adelic formulations of Propositions 1.5.24 and 1.5.34 and Lemma 1.5.29 are left to the reader. These are intermediate results; the principal applications (in $\S 3)$ will be stated in adelic form. In particular, the "long" calculation satisfies the analogue of Lemma 1.4.6.2. 


\section{CHAPTER 2}

\section{MIXED GROWTH CONDITIONS AND COHERENT COHOMOLOGY}

In this Chapter, we treat the conditions of moderate growth and rapid decrease on differential forms - and also combinations of the two - along divisors with normal crossings and discuss their implications on Dolbeault cohomology. In the case of the boundary divisor of a toroidal compactification of the mixed Shimura variety associated to the pair $(Q, X)$, we convert these conditions to growth and decay conditions on the corresponding vector-valued functions on the group $Q$ on various Siegel sets for its admissible parabolic subgroups. This enables us to deduce the useful isomorphism (2.7.1) on cohomology that seems inaccessible by geometric methods.

\section{1. $\bar{\partial}$ with logarithmic growth in one variable (cf. $[\mathrm{HP}],[\mathrm{H} 4, \S 3],[\mathrm{HZ} 1,3.8])$}

Let $f$ be a $C^{\infty}$ function on the punctured disc $\Delta_{a}^{*}=\{z \in \mathbb{C}: 0<|z| \leqslant a\}$, with $a<1$. Given an integer $N$, we say that $f$ has logarithmic growth of degree $\leqslant N$ when

$$
|f(z)||\log | z||^{-N}
$$

is bounded from above on $\Delta_{a}^{*}$. The set of all such functions is denoted $A_{N}^{0}$. It is obvious that $A_{N}^{0} \subset A_{N^{\prime}}^{0}$ for all $N^{\prime} \geqslant N$.

One says that $f$ is slowly increasing if it has logarithmic growth of degree $\leqslant N$ for some $N$, and rapidly decreasing if it has logarithmic growth of degree $\leqslant N$ for all $N .^{(1)}$ It is wise to restrict one's attention to functions $f$ such that the derivatives of $f$ of all orders are slowly increasing (resp. rapidly decreasing). The space of all such functions is denoted $A_{\text {sia }}^{0}$ (resp. $A_{\text {rda }}^{0}$ ).

If $\omega$ is a differential form of bidegree $(0,1)$, one can write $\omega=g(z) d \bar{z} / \bar{z}$. We say that $\omega$ has logarithmic growth of degree $\leqslant N$ whenever $g$ does; in effect, we declare that $d \bar{z} / \bar{z}$ is slowly increasing. We can then define the notion of $(0,1)$-forms that are

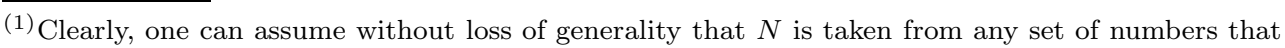
is unbounded from both above and below.
} 
slowly increasing or rapidly decreasing to all orders, and denote them by $A_{\text {sia }}^{(0,1)}$ and $A_{\text {rda }}^{(0,1)}$ respectively. We obtain Dolbeault complexes $A_{\text {sia }}^{\bullet}$ :

$$
A_{\mathrm{sia}}^{0} \stackrel{\bar{\partial}}{\longrightarrow} A_{\mathrm{sia}}^{(0,1)},
$$

and the analogously defined $A_{\text {rda }}^{\bullet}$, which is a subcomplex of $A_{\text {sia }}^{\bullet}$.

Proposition 2.1.2 (Dolbeault lemma with logarithmic growth)

(i) $H^{0}\left(A_{\text {sia }}^{\bullet}\right)$ is the space of holomorphic functions on the disc $\Delta_{a}$ of radius a.

(ii) $H^{0}\left(A_{\mathrm{rda}}^{\bullet}\right)$ is the space of holomorphic functions on $\Delta_{a}$ that vanish at the origin.

(iii) $H^{1}\left(A_{\text {rda }}^{\bullet}\right)=H^{1}\left(A_{\text {sia }}^{\bullet}\right)=0$.

As the first two assertions of the Proposition are obvious, we concentrate on (iii). This is seen to come down to verifying:

Proposition 2.1.3 ([HP]). - Let $N$ be an integer different from -1 . If $g \in A_{N}^{0}$, there exists $f \in A_{N+1}^{0}$ such that $\bar{\partial} f(z)=g(z) d \bar{z} / \bar{z}$.

For completeness, we give:

Outline of the proof of (2.1.3). - There is a standard solution of the $\bar{\partial}$-equation in a disc $\Delta$, which gets written here as $\bar{\partial} f / \partial \bar{z}=g(z) / \bar{z}$, viz. $f=I(g)$ where

$$
I(g)(z)=(2 \pi i)^{-1} \int_{w \in \Delta} \frac{g(w) d w \wedge d \bar{w} / \bar{w}}{w-z} .
$$

This is an absolutely convergent integral if $g$ has logarithmic growth; note that $I(g)$ is independent of $N$. One then shows that logarithmic growth for $g$ implies the corresponding growth for $f$. By decomposing (2.1.4) into pieces, as in [HP], one reduces the verification to the following elementary calculations:

LEmma 2.1.5. - Let $N$ be an integer different from -1. Then there is a constant $C_{N}>0$ such that whenever $r \leqslant a$ :

$$
\begin{gathered}
r^{-1} \int_{0}^{r}|\log \rho|^{N} d \rho \leqslant C_{N}|\log r|^{N}, \\
\int_{0}^{r} \rho^{-1}|\log \rho|^{N} d \rho \leqslant C_{N}|\log r|^{N+1}, \quad \text { if } N \geqslant 0 \\
\int_{r}^{a} \rho^{-1}|\log \rho|^{N} d \rho \leqslant C_{N}|\log r|^{N+1} \quad \text { if } N<0 .
\end{gathered}
$$

Moreover, when $g \in A_{\text {sia }}^{0}$ (resp. $\left.g \in A_{\text {rda }}^{0}\right)$, the formula

$$
\frac{\partial I(g)}{\partial z}=I\left(\frac{\partial g}{\partial z}\right)
$$

implies that $I(g) \in A_{\text {sia }}^{0}$ (resp. $\left.I(g) \in A_{\text {rda }}^{0}\right)$ likewise. 


\subsection{Logarithmic growth in several variables}

In the our setting, the natural notions of logarithmic growth and decay are the following. Given an integer $N$, a function $f$ of $(\boldsymbol{z}, \boldsymbol{w}) \in\left(\Delta_{a}^{*}\right)^{m} \times\left(\Delta_{a}\right)^{n}$ is said to have logarithmic growth of degree $\leqslant N$ when

$$
|f(\boldsymbol{z}, \boldsymbol{w})|\left(\sum_{j=1}^{m}|\log | z_{j}||\right)^{-N}
$$

is bounded from above. The set of all such functions is again denoted $A_{N}^{0}$. We can then define $A_{\text {sia }}^{0}$ and $A_{\text {rda }}^{0}$ as in (2.1). Also as in (2.1), we regard $d \bar{z}_{j} / \bar{z}_{j}$ as logarithmic (after all, it has logarithmic growth in the sense of (1.3.5)). It is not hard to see that these growth conditions allow one to define, on any complex manifold $M$, sheaves of forms having slow increase or rapid decrease along the divisor with normal crossings $D$, for the notion of logarithmic growth is independent of local coordinates, and likewise for all derivatives. We denote these by $\mathcal{A}_{\text {sia }}^{\bullet}(M, D)$ and $\mathcal{A}_{\text {rda }}^{\bullet}(M, D)$.

An alternate simpler notion of logarithmic growth of degree $N$ is provided by the boundedness of

$$
|f(\boldsymbol{z}, \boldsymbol{w})| \prod_{j=1}^{m}|\log | z_{j}||^{-N},
$$

which is asymptotically different from (2.2.1). However, (2.2.2) yields the same notions of slow increase or rapid decrease in view of the following:

Lemma 2.2.3. - If $\lambda_{j} \geqslant 1$ for $1 \leqslant j \leqslant m$, then

$$
\left(\prod_{j=1}^{m} \lambda_{j}\right)^{1 / m} \leqslant \frac{1}{m} \sum_{j=1}^{m} \lambda_{j} \leqslant \prod_{j=1}^{m} \lambda_{j} .
$$

This allow us to replace the growth condition of (2.2.1) by that of (2.2.2), for which we have, in effect, separation of variables (cf. [GH, p. 25]). One obtains from (2.2.3):

Proposition 2.2.4. - Let $D$ be a divisor with normal crossings on a complex manifold $M$. Then $\mathcal{A}_{\text {sia }}^{\bullet}(M, D)$ is a fine resolution of $\mathcal{O}_{M}$, and $\mathcal{A}_{\text {rda }}(M, D)$ is a fine resolution of $\mathcal{O}_{M}(-D)$.

Corollary 2.2.5. - Let $\mathcal{F}$ be a locally-free sheaf of $\mathcal{O}_{M}$-modules. Then the complex $\mathcal{A}_{\text {sia }}^{\bullet}(M, D) \otimes_{\mathcal{O}_{M}} \mathcal{F}$ is a fine resolution of $\mathcal{F}$, and $\mathcal{A}_{\text {rda }}^{\bullet}(M, D) \otimes_{\mathcal{O}_{M}} \mathcal{F}$ is a fine resolution of $\mathcal{F}(-D)$.

REMARK 2.2.6. - The issues involved in the proof of (2.2.4) were misrepresented in $[\mathrm{HP}]$, as was pointed out to us by J. I. Burgos, who also provided a correct argument along the lines outlined here. 


\subsection{Forms with mixed growth conditions}

We continue to let $D$ denote a divisor with normal crossings on the complex manifold $M$. Consider any 2-partition of the set of irreducible components of $D$, and write $D$ correspondingly as the union $D_{1} \cup D_{2}$. We define the middle term in

$$
\mathcal{A}_{\text {rda }}^{\bullet}(M, D) \subseteq \mathcal{A}_{\text {mxa }}^{\bullet}\left(M ; D, D_{2}\right) \subseteq \mathcal{A}_{\text {sia }}^{\bullet}(M, D)
$$

by the condition that a differential form should be slowly increasing along $D_{1}$, and rapidly decreasing along $D_{2}$, and likewise for all derivatives. Our purpose is to obtain the following variants of (2.2.4) and (2.2.5):

Proposition 2.3.2. - $\mathcal{A}_{\text {mxa }}^{\bullet}\left(M ; D, D_{2}\right)$ is a fine resolution of $\mathcal{O}_{M}\left(-D_{2}\right)$.

Corollary 2.3.3. - For any locally-free sheaf $\mathcal{F}$ of $\mathcal{O}_{M}$-modules, the complex $\mathcal{A}_{\text {mxa }}^{\bullet}\left(M ; D, D_{2}\right) \otimes_{\mathcal{O}_{M}} \mathcal{F}$ is a fine resolution of $\mathcal{F}\left(-D_{2}\right)$.

One is led to define:

Definition 2.3.4. - A function $f$ of $(\boldsymbol{z}, \boldsymbol{u}, \boldsymbol{w}) \in\left(\Delta_{a}^{*}\right)^{m_{1}} \times\left(\Delta_{a}^{*}\right)^{m_{2}} \times\left(\Delta_{a}\right)^{n}$ is said to have logarithmic growth of bidegree $\left(N_{1}, N_{2}\right)$ when

$$
|f(\boldsymbol{z}, \boldsymbol{u}, \boldsymbol{w})|\left(\sum_{j=1}^{m_{1}}|\log | z_{j}||\right)^{-N_{1}}\left(\sum_{j=1}^{m_{2}}|\log | u_{j}||\right)^{-N_{2}}
$$

is bounded from above. Differential $(0, k)$-forms of logarithmic growth of bidegree $\left(N_{1}, N_{2}\right)$ are defined just as before, and the space of such is denoted $A_{\left(N_{1}, N_{2}\right)}^{k}$.

Then Proposition (2.3.2) and indeed Proposition (2.2.4) are deduced from the following multivariate version of (2.1.3), which is proved by iteration on the latter.

Proposition 2.3.5. - Let $N_{1}$ and $N_{2}$ be integers different from -1 . If $k>0$ and $\omega \in A_{\left(N_{1}, N_{2}\right)}^{k}$, there exists $\phi \in A_{\left(N_{1}+1, N_{2}+1\right)}^{k-1}$ such that $\bar{\partial} \phi=\omega$.

\subsection{Siegel sets in mixed Shimura varieties}

We will retain the notation from (1.1) as far as possible. Thus, let $(Q, X)$ be a mixed Shimura datum. By convention (as before) we also allow $\mathcal{X}$ to denote a connected component of this homogeneous space. Let $(G, W \backslash X)$ be the corresponding pure Shimura datum for $G=Q / W$ and $\pi: Q \rightarrow G$ the natural map. For any connected component $M_{\Gamma}$ of $K_{f} \operatorname{Sh}(Q, \mathcal{X})(\mathbb{C})$ and corresponding component $\widehat{M}_{\Gamma}$ of $\widehat{K}_{f} \operatorname{Sh}(G, W \backslash X)(\mathbb{C})$, where $\widehat{K}_{f}=\pi\left(K_{f}\right)$, we have the commutative diagram of complex manifolds

$$
\begin{array}{cccc}
x & \longrightarrow & M_{\Gamma} \\
\downarrow & & \downarrow \\
\widehat{x}=W \backslash X & \longrightarrow & \widehat{M}_{\Gamma}
\end{array}
$$


in which the spaces $X$ and $\widehat{x}$ are contractible.

The notion of going to infinity in $M_{\Gamma}$ or $\widehat{M}_{\Gamma}$ is expressible in terms of Siegel sets, equivalently in the group or on the homogeneous space, associated to parabolic subgroups. For the present purposes, it is the admissible parabolic subgroups that enter. Recall from (1.1.9) that the mapping $\mathcal{P}(G) \rightarrow \mathcal{P}(Q)$ that assigns $\widetilde{P}=\pi^{-1}(P)$ to $P \in \mathcal{P}(G)$ is bijective. Since the notion of admissibility is determined on $G$, the admissible parabolic subgroups of $G$ and $Q$ are thus in canonical one-to-one correspondence.

We work with the pair $(G, \widehat{X})$, though the story for $(Q, X)$ is parallel. Let $P \in \mathcal{P}(G)$; then $P(\mathbb{R})$ acts transitively on $\widehat{x}$. Select a basepoint $\widehat{x}_{0} \in \widehat{x}$. Let $A_{P}$ denote the associated lift to $P(\mathbb{R})$ of the connected component of the center of $P / W_{P}$. Then $P^{0} \simeq\left({ }^{0} P\right)^{0} \times A_{P}$, where ${ }^{0} P$ denotes, as in [BS, 1.1], the intersection of the kernels of the squares of the rational characters of $P$; we make this identification routinely. A Siegel set for $P$ in $G(\mathbb{R})$ is a subset of $P(\mathbb{R})$ of the form $\mathfrak{S}=\mathfrak{S}_{\kappa, t}=\kappa \times A_{P, t}$, where $\kappa$ is a compact subset of ${ }^{0} P(\mathbb{R})$, and

$$
A_{P, t}=\left\{a \in A_{P}: a^{\beta} \geqslant t \text { for all simple roots } \beta \text { occurring in } W_{P}\right\} .
$$

It is also standard usage to call $\mathfrak{S} \cdot \widehat{x}_{0}$ a Siegel set for $P$ in $\widehat{x}$.

In what follows, $U_{P}$ is the center of the unipotent radical $W_{P}$ of $P, V_{P}=W_{P} / U_{P}$. The associated Siegel domain coordinates are deduced from embedding $\widehat{x}$ as an open subset of the total space $\widehat{x}_{P}$ of a mixed Shimura variety,

$$
\widehat{x} \subset \widehat{x}_{P} \longrightarrow U_{P}(\mathbb{C}) \backslash \widehat{\mathcal{X}}_{P} \longrightarrow \widehat{F}_{P},
$$

where $\widehat{F}_{P}$ is the boundary component of $\widehat{x}$ corresponding to $P$, and trivializing the fibration analytically:

$$
\widehat{x}_{P} \simeq \widehat{F}_{P} \times V_{P}(\mathbb{R}) \times U_{P}(\mathbb{C})
$$

This yields

$$
\widehat{x} \simeq\left\{(y, v, u) \in \widehat{F}_{P} \times V_{P}(\mathbb{R}) \times U_{P}(\mathbb{C}): \operatorname{Im} u-\Psi(y, v) \in C_{P}\right\},
$$

where $C_{P}$ is a homogeneous cone in $U_{P}(\mathbb{R})$, in which the action of $A_{P}$ on $\widehat{x}$ is given by the dilations of $C_{P}$. In the above, $\Psi: \widehat{F}_{P} \times V_{P}(\mathbb{R}) \rightarrow U_{P}(\mathbb{R})$ has well-known properties; aside from continuity, these will not concern us here.

Remark 2.4.6. - When $G$ is $\mathbb{Q}$-simple and $P$ is maximal, one places a point at infinity in $V_{P}(\mathbb{R}) \times U_{P}(\mathbb{C})$ in $(2.4 .2)$ to attach (topologically) the Baily-Borel boundary component corresponding to $P$. It is reached by letting $a^{\beta} \rightarrow \infty$ (see (2.4.2)), while keeping the simple roots of $G_{h, P}$ bounded.

Next, let $\mathcal{T}$ be any compact cone in $C_{P}$; by "compact", we mean that $\widehat{\mathcal{T}}=(\mathcal{T}-0) / \mathbb{R}^{+}$ is compact in $\widehat{C}_{P}:=\left(C_{P}-0\right) / \mathbb{R}^{+}$. A complex Siegel set for $P$ in $\widehat{X}$, built from $\mathcal{T}$, 
will be a set $S$ of the form

$$
\left\{(y, v, u) \in \widehat{F}_{P} \times V_{P} \times U_{P}(\mathbb{C}): y \in \kappa_{h}, v \in \kappa_{V}, \operatorname{Re} u \in \kappa_{U}, \operatorname{Im} u \in\left(t_{0}+\mathcal{T}\right)\right\}
$$

for $\kappa_{h}, \kappa_{V}, \kappa_{U}$ compact and $t_{0} \in \mathcal{T}$. It is easy to verify that the two notions of Siegel set are commensurate: every Siegel set for $P$ is contained in a complex Siegel set for $P$, and conversely. (For the same reason, one sees that in defining a Siegel set, one could take the $\kappa$ to be a compact subset of $P(\mathbb{R})$ or even of $G(\mathbb{R})$.)

We turn now briefly to the pair $(Q, X)$. As our current concern is over mixed Shimura varieties for which $\pi_{2}$ in (1.1.5) is an isomorphism (that is to say, abelian fibrations), we assume that $W_{-2} Q=\{1\}$. Let $\widetilde{P} \in \mathcal{P}(Q)$ correspond to $P \in \mathcal{P}(G)$. There are objects for $\widetilde{P}$ analogous to those for $P$ given above, as defined in $[\mathrm{P}, \S 4]$. This yields a diagram:

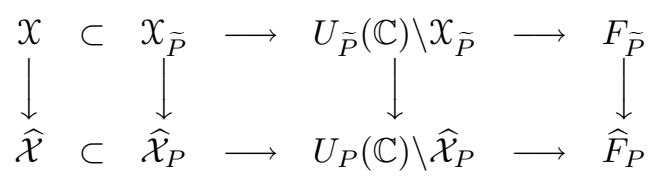

in which the vertical arrows are surjections, and a surjection of cones $C_{\widetilde{P}} \rightarrow C_{P}$. (Note, however, that the homomorphism $U_{\widetilde{P}} \rightarrow U_{P}$ need not be injective.) Moreover, if we select a basepoint $x_{0} \in X$ with $\pi\left(x_{0}\right)=\widehat{x}_{0}$, the projection of $A_{\widetilde{P}}$ in $Q(\mathbb{R})$ to $A_{P} \subset G(\mathbb{R})$ is an isomorphism. Thus Siegel sets for $\widetilde{P}$ in $\mathcal{X}$, defined in the same way as those for $P$, map onto Siegel sets for $P$ in $\widehat{X}$. In actuality, though, it will suffice for our purposes to consider the Siegel sets for $P$ and $\widetilde{P}$ independently.

The following is well-known (see [B3]):

Proposition 2.4.9. - Let $\kappa$ be a compact subset of ${ }^{0} P(\mathbb{R}), \mathfrak{S}_{\kappa, t}$ the Siegel set $\kappa \times A_{P, t}$ and $\left(\mathfrak{S}_{\kappa, t}\right)_{\Gamma}$ the image of $\mathfrak{S}_{\kappa, t}$ in $\widehat{M}_{\Gamma} \subset \widehat{K}_{f} \operatorname{Sh}(G, \widehat{\mathcal{X}})(\mathbb{C})$. Then for $t$ sufficiently large,

$$
\left(\mathfrak{S}_{\kappa, t}\right)_{\Gamma} \simeq \kappa_{\Gamma} \times A_{P, t},
$$

where $\kappa_{\Gamma}$ is the image of $\kappa$ in $\widehat{M}_{\Gamma}$, such that the projection $\mathfrak{S}_{\kappa, t} \rightarrow\left(\mathfrak{S}_{\kappa, t}\right)_{\Gamma}$ decomposes as $\left(\kappa \rightarrow \kappa_{\Gamma}\right) \times \mathbf{1}_{A_{P, t}}$.

Corollary 2.4.10. - Let $\left(\mathfrak{S}_{\kappa, t}\right)_{\Gamma}$ be as in (2.4.9). Then for $t$ sufficiently large, there is a canonical projection $a:\left(\mathfrak{S}_{\kappa, t}\right)_{\Gamma} \rightarrow A_{P}\left(A_{P}\right.$-coordinate $)$.

\subsection{Toroidal compactifications and Siegel sets}

For simplifying notation only, we assume henceforth that $G$ is $\mathbb{Q}$-simple. We leave it to the reader to formulate the general case. 
We start by taking a slight variation of the basic diagram (induced by (2.4.3)) in [HZ2, 1.4.4]:

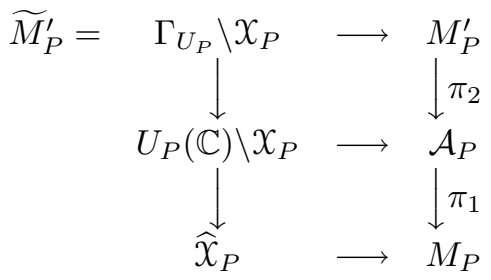

(In [HZ1] $\mathcal{A}_{P}$ was denoted $A_{F}$ or $A_{P}$, but here $A_{P}$ has a different meaning.) We have made the upper square cartesian: $\widetilde{M}_{P}^{\prime}$ is the pullback of $M_{P}^{\prime}$ to the universal cover $U_{P}(\mathbb{C}) \backslash X_{P}$ of $\mathcal{A}_{P}$, replacing $X_{P}$. The coordinates in (2.4.4) induce an analytic isomorphism

$$
\widetilde{M}_{P}^{\prime} \simeq \widehat{X}_{P} \times V_{P}(\mathbb{R}) \times T_{P}
$$

where $T_{P}$ denotes $U_{P}(\mathbb{Z}) \backslash U_{P}(\mathbb{C})$, viewed as the complex points of the torus with cocharacter group $U_{P}(\mathbb{Z})$.

We next review some pertinent features of the structure of the boundary of toroidal compactifications $M_{\Gamma, \Sigma}$ of connected components of $\operatorname{Sh}(G, \mathcal{X})(\mathbb{C})$, following $\left[\right.$ HZ1, (1.5)]. Let $P \in \mathcal{P}(G)$. The data in $\Sigma_{P}$ are used to construct $\pi:\left(M_{P}^{\prime}\right)_{\Sigma_{P}} \rightarrow M_{P}$, with boundary divisor written as

$$
<\widetilde{Z}_{\Sigma}(\succeq P) \stackrel{\text { def }}{=} \bigcup_{P^{\prime} \succeq P}<\widetilde{Z}_{\Sigma}\left(P^{\prime}\right) .
$$

The quotient of (2.5.3) by $\Gamma_{\ell, P}$ will be denoted $<Z_{\Sigma}(\succeq P)$; in the notation of [HZ1] it is the union of all $<Z_{\Sigma}\left(P^{\prime}\right)$ for $P^{\prime} \succeq P$. Recall that a neighborhood of the latter is a building block in the construction of $M_{\Gamma, \Sigma}$. It will be useful to keep in mind that $<Z_{\Sigma}(\succeq P)$ is more than the $P$-stratum of the boundary unless $P$ is maximal with respect to $\succ$, yet contains all of $Z_{\Sigma}(P)$ (the closure of the $P$-stratum) only when $P$ is minimal with respect to $\succ$. We also observe that $T_{P}$ acts on (2.5.3).

The toroidal constructions with $T_{P}$ lift to ones on $\widetilde{M}_{P}^{\prime}$. In particular, we have

$$
\left(\widetilde{M}_{P}^{\prime}\right)_{\Sigma_{P}} \simeq \widehat{\mathcal{X}}_{P} \times V \times\left(T_{P}\right)_{\Sigma_{P}}
$$

Let $<\widetilde{\widetilde{Z}}_{\Sigma}(P)$ and $<\widetilde{\widetilde{Z}}_{\Sigma}(\succeq P)$ denote the respective pullbacks of $<\widetilde{Z}_{\Sigma}(P)$ and $<\widetilde{Z}_{\Sigma}(\succeq P)$; we can write, for instance,

$$
<\widetilde{\widetilde{Z}}_{\Sigma}(\succeq P) \simeq \widehat{x}_{P} \times V \times \partial\left(T_{P}\right)_{\Sigma_{P}} .
$$

In $[\mathrm{HZ2}, 1.4(\mathrm{~d})]$, we introduced a topological compactification of $M_{\Gamma}$, the real boundary quotient $M_{\Gamma, \Sigma, \partial_{\mathbb{R}}}$ of $M_{\Gamma, \Sigma}$, which was designed for the present discussion (also, cf. [HZ1, 2.3]), with structure map

$$
p: M_{\Gamma, \Sigma} \longrightarrow M_{\Gamma, \Sigma, \partial_{\mathbb{R}}}
$$


By construction, $p\left(<Z_{\Sigma}(\succeq P)\right)$ is given naturally as $\Gamma_{\ell, P} \backslash\left(<\widetilde{Z}_{\Sigma}(\succeq P) / T_{P}^{c}\right)$, where $T_{P}^{c}$ is the maximal compact real torus in $T_{P}$. Likewise, we can define the real boundary quotient

$$
\left(\widetilde{M}_{P}^{\prime}\right)_{\Sigma_{P}, \partial_{\mathbb{R}}} \simeq \widehat{X}_{P} \times V \times \partial\left(T_{P}\right)_{\Sigma_{P}, \partial_{\mathbb{R}}}
$$

for which $\partial\left(\widetilde{M}_{P}^{\prime}\right)_{\Sigma_{P}, \partial_{\mathbb{R}}} \simeq \widehat{x}_{P} \times V \times\left(T_{P}\right)_{\Sigma_{P}, \partial_{\mathbb{R}}}$. In terms of the product structures in (2.5.4) and (2.5.6), the quotient map $\left(\widetilde{M}_{P}^{\prime}\right)_{\Sigma_{P}} \rightarrow\left(\widetilde{M}_{P}^{\prime}\right)_{\Sigma_{P}, \partial_{\mathbb{R}}}$ is induced by the natural projection $\left(T_{P}\right)_{\Sigma_{P}} \rightarrow\left(T_{P}\right)_{\Sigma_{P}, \partial_{\mathbb{R}}}$.

For any character $\chi$ of $T_{P}$, there is a morphism of commutative diagrams ${ }^{(2)}$, which we also denote by $\chi$ :

$$
\begin{aligned}
& \left\{\begin{array}{ccc}
U_{P}(\mathbb{C}) & \stackrel{\operatorname{Im}}{\longrightarrow} U_{P}(\mathbb{C}) / U_{P}(\mathbb{R}) \simeq U_{P}(\mathbb{R}) \\
\exp \downarrow & \exp \downarrow \simeq \\
U_{P}(\mathbb{C}) / U_{P}(\mathbb{Z}) \simeq T_{P} & & \\
\operatorname{Mod} & T_{P} / T_{P}^{c}
\end{array}\right\} \\
& \left\{\begin{array}{ccc}
\mathbb{C} & \stackrel{\operatorname{Im}}{\longrightarrow} & \mathbb{C} / \mathbb{R} \simeq \mathbb{R} \\
\exp \downarrow & \exp \downarrow \\
\mathbb{C} / \mathbb{Z} \simeq \mathbb{C}^{*} \underset{\operatorname{Mod}}{\longrightarrow} \mathbb{C}^{*} / S^{1} \simeq \mathbb{R}^{+}
\end{array}\right\}
\end{aligned}
$$

In other words, up to a constant multiple, the logarithm of the modulus of a toroidal variable is induced by the imaginary part of a linear function on $U_{P}(\mathbb{C})$. In this way, approaching the boundary of $\widetilde{X}_{\Sigma, \partial_{\mathbb{R}}}$ corresponds to going to infinity in the radial direction of the cone $C_{P}$. Specifically, $\partial\left(T_{P}\right)_{\Sigma_{P}, \partial_{\mathbb{R}}}$ can be viewed as a copy of $\widehat{\Sigma}_{P}$ attached at infinity to the cone, with $\widehat{\Sigma}_{P}^{(1), c}$ giving $p\left(<Z_{\Sigma}(P)\right)$ (see the illustration $[\mathrm{HZ1},(2.3 .10)])$, while $T_{P}^{c}$-orbits are getting collapsed to a point.

We make the following:

ObServations 2.5.8. - Let $P$ be a maximal $\mathbb{Q}$-parabolic subgroup of $G, \mathcal{T}$ a compact cone in $C_{P}$, and $S$ a complex Siegel set for $P$ built from $\mathcal{T}$, as in (2.4.7). Then:

(i) the closure of the image of $S$ in $\left(\widetilde{M}_{P}^{\prime}\right)_{\Sigma_{P}, \partial_{\mathbb{R}}}$ meets the boundary in $\kappa_{h} \times \kappa_{V} \times \mathcal{T}$,

(ii) Take $\mathcal{T}$ to be any union of finitely many top-dimensional simplicial cones in $\Sigma_{P}^{(1), c}$ that maps onto $\Gamma_{\ell, P} \backslash \Sigma_{P}^{(1), c}$, and assume that $\kappa_{V}$ and $\kappa_{U}$ are sufficiently large. Then the closure of the image of $S$ in $M_{\Gamma, \Sigma}$ meets the boundary in $\pi^{-1}\left(\widehat{\kappa}_{h}\right) \cap<Z_{P}$, where $\widehat{\kappa}_{h}$ denotes the image of $\kappa_{h}$ in $M_{P}$.

(iii) For any $P^{\prime} \succ P$, there is a dense open subset (determined by $\mathcal{Y}_{P}\left(P^{\prime}\right)$ below) of $Z_{\Sigma}\left(P^{\prime}\right)$ of which each point is in the closure of some Siegel set for $P$.

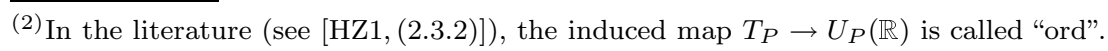


In practice, the above asserts that every point of $Z_{\Sigma}(P)$ (the union of components of the toroidal divisor generated by $\Sigma_{P}^{c}$ ) is a limit point of some Siegel set; however, one must consider Siegel sets for all parabolic subgroups dominated by (i.e., $\prec$ ) $P$, as well as those for $P$ itself.

To be more specific, if we let $V(L)$ stand for the set of vertices of a simplicial complex $L$, then

$$
V\left(\widehat{\Sigma}_{P}\right)=\coprod\left\{V\left(\widehat{\Sigma}_{P^{\prime}}^{c}\right): P^{\prime} \succeq P\right\}
$$

is a $\Gamma_{\ell, P}$-equivariant partition (cf. $[\mathrm{HZ} 2,(2.4 .2)]$ ). By taking the real projection of the toroidal divisors corresponding to each $\widehat{\Sigma}_{P^{\prime}}^{c}$, one induces a decomposition of $\widehat{C}_{P}$ (which is an open subset of $\left.\left|\widehat{\Sigma}_{P}\right|\right)$ into sets $\widehat{\mathcal{Y}}_{P}\left(P^{\prime}\right)$ that intersect only at their boundaries, and which are the closure of their interiors; of these, only $\widehat{\mathcal{Y}}_{P}(P)$ is compact modulo $\Gamma_{\ell, P}$. In $[\mathrm{HZ} 2,(2.2)], \widehat{\mathcal{Y}}_{P}\left(P^{\prime}\right)$ gets called the open thick $P^{\prime}$-stratum of $\widehat{\Sigma}_{P}^{(1)}$, and the decomposition (2.5.9) plays an important role. When $\mathcal{T} \subset C_{P}$ as before, we will say that $\mathcal{T}$ abuts on $\widetilde{Z}_{\Sigma}\left(P^{\prime}\right)$ whenever $\widehat{\mathcal{T}} \subset \widehat{\mathcal{Y}}_{P}\left(P^{\prime}\right)$. Letting $\mathcal{Y}_{P}\left(P^{\prime}\right)$ denote the corresponding cone in $C_{P}$, we conclude:

Proposition 2.5.10. - Let $P$ be a maximal $\mathbb{Q}$-parabolic subgroup of $G$. Then:

(i) The set of all complex Siegel sets for admissible parabolics that abut on $\widetilde{Z}_{\Sigma}(P)$ consists precisely of those complex Siegel sets, for some $Q \preceq P$, built from compact cones contained in the subcone $\mathcal{Y}_{Q}(P)$ of $C_{Q}$.

(ii) Let $\widetilde{Z}_{\Sigma}^{Q}(P)$ denote the union of the limit points of such Siegel sets for a fixed $Q$. Then this set is open and dense in $\widetilde{Z}_{\Sigma}(P)$.

(iii) Let $Z_{\Sigma}^{Q}(P) \subseteq Z_{\Sigma}(P)$ denote the image of $\widetilde{Z}_{\Sigma}^{Q}(P)$ in $M_{\Gamma, \Sigma}$. Then

$$
Z_{\Sigma}(P)=\bigcup\left\{Z_{\Sigma}^{Q}(P): Q \in \mathcal{M}(\Gamma)\right\}
$$

where $\mathcal{M}(\Gamma)$ is a set of $\Gamma$-conjugacy classes of admissible parabolic subgroups $Q$ with $Q \preceq P$.

(iv) $\bigcap\left\{Z_{\Sigma}^{Q}(P): Q \in \mathcal{M}(\Gamma)\right\}$ is an open and dense subset of $Z_{\Sigma}(P)$. It is open in the Zariski topology only when $P$ is minimal with respect to $\preceq$, i.e., when $\mathcal{M}(\Gamma)$ has only one element (representable by $\{P\}$ ).

\subsection{Growth conditions at the toroidal boundary}

Given $P \in \mathcal{P}(G)$, let $\beta$ denote the positive simple root occurring in $W_{P}$. We now prove:

Proposition 2.6.1. - Let $(\boldsymbol{z}, \boldsymbol{w}) \in\left(\Delta_{r}^{*}\right)^{n} \times \Delta_{r}^{m}$, with $r<1$, give local coordinates on a deleted neighborhood of ${ }^{<} Z_{\Sigma}(\succeq P)$ associated to an n-fold intersection of components of this divisor. Then on the intersection of this coordinate neighborhood with $\left(\mathfrak{S}_{\kappa, t}\right)_{\Gamma}$, the functions $a^{\beta}$ (determined by (2.4.10)) and $\sum_{j=1}^{n}|\log | z_{j}||$ grow at the same rate along $<Z_{\Sigma}(\succeq P)$. 
An argument proving this is given for pure Shimura varieties in [Mu, p. 264], though it seems a bit unfocused. The proof is geometric, and goes the same in the mixed case; we present it here:

Proof. - We appeal to (2.5.7). Write $\lambda_{j}$ for $|\log | z_{j}||$; these are linear functions on the cone that are positive in the region under consideration. We may assume without loss of generality that the Siegel set $\left(\mathfrak{S}_{\kappa, t}\right)_{\Gamma}$ involves, as a factor, a compact subset of the "standard" cross-section of $C_{P}-\{0\} \rightarrow \widehat{C}_{P}$ determined by $G_{\ell, P}(\mathbb{R})^{\text {der }}$. Then, the value of $a^{\beta}$ at any point is given by the amount of dilation in $C_{P}$ required to get to the point from this cross-section. By compactness, this differs from the amount of dilation required to get from any other cross-section over the same compact set by an amount that is bounded from above and away from zero. In other words, the rate of growth of the $A_{P}$-coordinate is independent of cross-section. Then, just take the cross-section to be the one defined by $\sum_{j} \lambda_{j}=1$.

Next, let $\widehat{x}_{0}$ be the basepoint of $\widehat{x}$, which was used in definition of Siegel sets in (2.4). Let $\widehat{K}_{0}$ denote the stabilizer in $G(\mathbb{R})^{\text {der }}$ of $\widehat{x}_{0}$. It is well-known (see [B1]) that there are norms $\|\cdot\|$ on $G(\mathbb{R})^{\text {der }}$ that satisfy:

(i) $\|g k\|=\|g\|$ whenever $k \in \widehat{K}_{0}$;

(ii) $\left\|g g^{\prime}\right\| \leqslant\|g\|\left\|g^{\prime}\right\|$.

The following is easy to verify:

Proposition 2.6.2. - Let $P$ be a maximal parabolic subgroup of $G$. Then:

(i) On $A_{P},\|a\|$ grows like $\left(a^{\beta}\right)^{\ell}$ for some $\ell>0$;

(ii) For $g$ in the Siegel set $\mathfrak{S}_{\kappa, t},\|g\|$ and $\|a(g)\|$ have the same rate of growth.

We recall that a $C^{\infty}(0, q)$-form $\omega$ on $M_{\Gamma}$ with values in $\mathcal{W}$ is determined by a unique smooth $\Gamma$-invariant function $f_{\omega}$ on $G(\mathbb{R})$ with values in $\bigwedge^{q}\left(\mathfrak{p}^{-}\right)^{*} \otimes W$. When we combine the preceding with (2.5.10), we get:

Proposition 2.6.3

(i) An element $\omega$ of the Dolbeault complex for $\mathcal{W}$ on (an open subset of) $M_{\Gamma}$ has moderate growth along $Z_{\Sigma}(P)$ in the sense of (2.2.4) if and only if $f_{\omega}$ has moderate growth on all complex Siegel sets for $Q$ that are built from subcones of $\mathcal{Y}_{Q}(P) \subset C_{Q}$, for all $Q \preceq P$.

(ii) $\omega$ lies in $\mathcal{A}_{m x a}^{\bullet}\left(M_{\Gamma, \Sigma} ; Z_{\Sigma}, Z_{\Sigma}(P)\right) \otimes \mathcal{W}^{\text {can }}$ (of (2.3)) if and only if $f_{\omega}$ has moderate growth and has rapid decrease on all complex Siegel sets for $Q$ that are built from subcones of $\mathcal{Y}_{Q}(P) \subset C_{Q}$, for all $Q \preceq P$.

\subsection{Mixed growth conditions and basechange}

We now take in (2.4.1) the lower part of the tower for the mixed Shimura variety for the admissible parabolic subgroup $P$ of $G$ (this is denoted $\pi_{1}: \mathcal{A}_{P} \rightarrow M_{P}$ in 
(2.5.1)). According to $[\mathrm{P}]$, there exist compatible toroidal compactifications for $\pi_{1}$. Let $\widetilde{\pi}_{1}: \widetilde{\mathcal{A}}_{P, \Xi} \rightarrow \widetilde{M}_{P, \Xi}($ as in $[\mathrm{HZ} 1,1.6])$ be one of them. We let $Z_{\Xi}^{A}\left(P^{\prime}\right)$ and $Z_{\Xi}\left(P^{\prime}\right)$ resp. denote the $P^{\prime}$-strata of the respective toroidal boundaries. The main goal of this section is to deduce the following variant and extension of [HZ1, 3.14]:

Proposition 2.7.1. - In the above situation, there is a commutative diagram of quasi-isomorphisms of sheaves on $\widetilde{M}_{P, \Xi}$ :

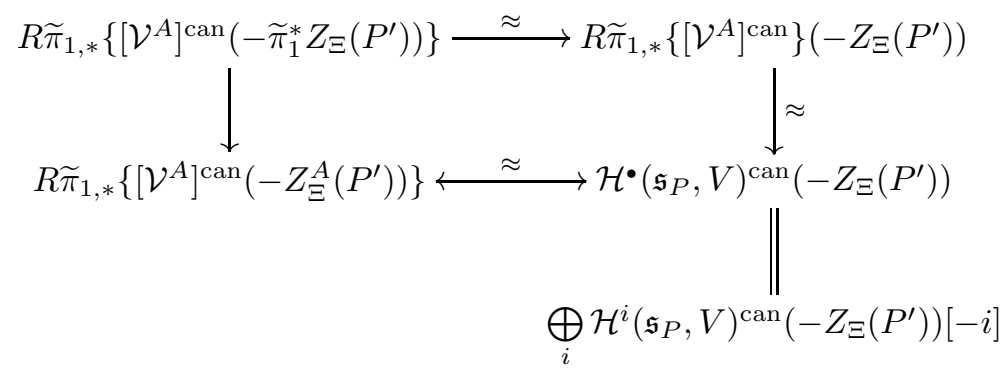

This implies at once:

COROLlary 2.7.2. - In the above situation,

$$
H^{\bullet}\left(\widetilde{A}_{P, \Xi},\left[\mathcal{V}^{A}\right]^{\operatorname{can}}\left(-Z_{\Xi}^{A}\left(P^{\prime}\right)\right)\right) \simeq H^{\bullet}\left(\widetilde{M}_{P, \Xi}, \mathcal{H}^{\bullet}\left(\mathfrak{s}_{P}, V\right)^{\operatorname{can}}\left(-Z_{\Xi}\left(P^{\prime}\right)\right)\right) .
$$

REMARK 2.7.3. - There would be nothing to prove in (2.7.1) if one knew that $Z_{\Xi}^{A}\left(P^{\prime}\right)$ were equal to $\widetilde{\pi}_{1}^{*} Z_{\Xi}\left(P^{\prime}\right)$ in the sense of divisors, i.e., that the fibers of $\widetilde{\pi}_{1}$ over $Z_{P^{\prime}}$ are reduced. One might hope that this can be arranged by choosing $\Xi$ suitably, but that is not apparent from $[\mathrm{P}]$.

Proof of (2.7.1). - We show first that in the derived category of $\widetilde{M}_{P, \Xi}$, both sides of the isomorphism in the bottom row of (2.7.1), are computable by means of complexes of $C^{\infty}$ differential forms, on $M_{P}$ and $\mathcal{A}_{P}$ respectively, with moderate growth and that decrease rapidly in the direction of the $P^{\prime}$-strata.

We use the mechanism from [HZ1, 3.5.12]. Consider the Dolbeault complexes:

$$
\mathcal{A}_{M_{P}}^{0, \bullet}\left(\mathcal{H}^{\bullet}\left(\mathfrak{s}_{P}, V\right)\right) \stackrel{\iota_{1}}{\longrightarrow} \mathcal{A}_{M_{P}}^{0, \bullet}\left(\bigwedge \mathfrak{s}_{P}^{*} \otimes \mathcal{V}\right) \stackrel{\iota_{2}}{\longleftrightarrow} \pi_{1 *} \mathcal{A}_{A_{P}}^{0, \bullet}\left(\mathcal{V}^{A}\right)
$$

up to quasi-isomorphism, this is

$$
\mathcal{H}^{\bullet}\left(\mathfrak{s}_{P}, V\right) \longrightarrow \bigwedge \mathfrak{s}_{P}^{*} \otimes \mathcal{V} \longrightarrow R \pi_{1, *} \mathcal{V}^{A} .
$$

The complexes in (2.7.4) can be written as sheaves of relative Lie algebra cochains:

$$
\mathcal{C}_{1}^{\bullet} \mathcal{C}_{2}^{\bullet} \longleftrightarrow \mathcal{C}_{3}^{\bullet},
$$

where, for $O$ open in $M_{P}$,

$$
\begin{aligned}
& \mathcal{C}_{1}(O)=\left\{C^{\infty}\left(O_{h, P}\right) \otimes \bigwedge\left(\mathfrak{p}_{h}^{-}\right)^{*} \otimes H^{\bullet}\left(\mathfrak{s}_{P}, V\right)\right\}^{K_{h, P},} \\
& \mathcal{C}_{2}(O)=\left\{C^{\infty}\left(O_{h, P}\right) \otimes \bigwedge\left(\mathfrak{p}_{h}^{-}\right)^{*} \otimes \bigwedge\left(\mathfrak{s}_{P}\right)^{*} \otimes V\right\}^{K_{h, P}},
\end{aligned}
$$


where $O_{h, P}$ is the inverse image of $O$ under $\Gamma_{h, P} \backslash G_{h, P} \rightarrow M_{P}$; also for $O^{\prime}$ the inverse image of $O$ in $A_{P}$

$$
\mathcal{C}_{3}^{\bullet}(O)=\left\{C^{\infty}\left(O_{1}^{\prime}\right) \otimes \bigwedge\left(\mathfrak{p}_{h}^{-} \oplus \mathfrak{s}_{P}\right)^{*} \otimes V\right\}^{K_{h, P}},
$$

with $O_{1}^{\prime}$ the inverse image of $O_{h, P}$ in $\Gamma_{A_{P}} \backslash\left(P^{\prime} / U_{P}\right)(\mathbb{R})$. For (2.7.6.3), we are using the fact that $\mathcal{V}^{A}$ is a homogeneous vector bundle (recall (1.2.14)), determined by the representation of $K_{h, P}$ on $H^{0}\left(T_{P}, \mathcal{V}_{P}^{\prime}\right)^{T_{P}}$, which is isomorphic (via evaluation at the basepoint) to $V$ as a $K_{h, P}$-module.

There are homotopy inverses to the maps in (2.7.6) via projections

$$
\mathcal{C}_{1} \stackrel{\psi_{1}}{\longleftarrow} \mathcal{C}_{2}^{\bullet} \stackrel{\psi_{2}}{\longleftarrow} \mathcal{C}_{3}^{\bullet}
$$

with $\psi_{1}$ given by the semi-simplicity of representations of a compact group - note that we are invoking here the assumption that $\mathfrak{p}^{-}$acts trivially on $V$-and $\psi_{2}$ given by taking the constant term along the fibers of $\mathcal{A}_{P}$.

Here and again, one makes use of the following homological lemma:

Lemma 2.7.8. - Let $\mathcal{C}_{1}$ and $\mathcal{C}_{2}$ be cochain complexes [of sheaves], and assume given morphisms $i: \mathcal{C}_{1} \rightarrow \mathcal{C}_{2}$ and $\psi: \mathcal{C}_{2} \rightarrow \mathcal{C}_{1}$ such that

(i) $\psi \circ i=1$,

(ii) $i \circ \psi=1+d B+B d$.

for some mapping $B: \mathcal{C}_{2} \rightarrow \mathcal{C}_{1}$ of degree -1 . Let $\mathcal{C}_{2}^{\prime}=\operatorname{Ker} \psi$. If $i^{\prime}: \mathcal{C}_{2}^{\prime} \rightarrow \mathcal{C}_{2}$ denotes the inclusion, the morphism

$$
\mathcal{C}_{1} \oplus \mathcal{C}_{2}^{\prime} \stackrel{i \oplus i^{\prime}}{\longrightarrow} \mathcal{C}_{2}
$$

is an isomorphism. Moreover, $\mathcal{C}_{2}^{\prime} \approx 0$, and $i$ and $\psi$ are quasi-isomorphisms inducing mutually inverse isomorphisms on cohomology [sheaves]. Furthermore, the above is functorial with respect to the triple $(i, \psi, B)$.

This yields that the maps in (2.7.4), and therefore also (2.7.5), are quasi-isomorphisms. This was extended to the toroidal boundary for the canonical extension in [HZ1,3.13] and the subcanonical extension in [HZ1, 3.14]. These were obtained by observing that $\psi_{1}$ and $\psi_{2}$ respect the growth conditions on (2.7.7) given as in (2.6.1), as do their homotopy operators, and then appealing to (2.7.8). Said another way, imposing the growth conditions preserved the homological situation, and we could again invoke (2.7.8).

We apply (2.3.3) to both sides of bottom row in (2.7.1), writing them as Dolbeault complexes with mixed growth conditions on $\widetilde{\mathcal{A}}_{P, \Xi}$ and $\widetilde{M}_{P, \Xi}$ resp., taking $D_{2}$ to be the respective $P^{\prime}$-stratum of the toroidal boundary, for some $\mathbb{Q}$-parabolic subgroup $Q$ 
of $P$. This reduces us to verifying that

$$
\begin{aligned}
\mathcal{A}_{\mathrm{mxa}}^{\bullet}\left(\widetilde{M}_{P, \Xi} ; Z_{\Xi}, Z_{\Xi}\left(P^{\prime}\right)\right) \otimes & \mathcal{H}^{\bullet}\left(\mathfrak{s}_{P}, V\right)^{\mathrm{can}} \\
& \longrightarrow \widetilde{\pi}_{1, *}\left\{\mathcal{A}_{\mathrm{mxa}}^{\bullet}\left(\widetilde{\mathcal{A}}_{P, \Xi} ; Z_{\Xi}^{A}, Z_{\Xi}^{A}\left(P^{\prime}\right)\right) \otimes\left[\mathcal{V}^{A}\right]^{\text {can }}\right\} .
\end{aligned}
$$

is a quasi-isomorphism, extending that of (2.7.4) to the toroidal boundary. For use in conjunction with the Lie algebra cochains of (2.7.6), we appeal to (2.6.3)(ii): the mixed growth conditions are equivalent to moderate growth, together with decay conditions on a specific class of Siegel sets. That these are preserved by $\psi_{1}$ and $\psi_{2}$ in (2.7.7) follows as before. This gives that (2.7.9) is a quasi-isomorphism, and (2.7.1) follows.

To complete the proof of (2.7.1), we must show that the square commutes. First, we make the following observation, which follows from (2.2.5) and (2.3.3):

Lemma 2.7.10. - The inclusions

$$
\begin{aligned}
& \mathcal{A}_{\text {sia }}^{\bullet}\left(\widetilde{\mathcal{A}}_{P, \Xi}, Z_{\Xi}^{A}\right) \otimes\left[\mathcal{V}^{A}\right]^{\text {can }} \otimes \widetilde{\pi}^{*} \mathcal{I}_{Z_{\Xi}\left(P^{\prime}\right)} \\
& \longleftrightarrow \mathcal{A}_{\text {mxa }}^{\cdot}\left(\widetilde{\mathcal{A}}_{P, \Xi} ; Z_{\Xi}^{A}, Z_{\Xi}^{A}\left(P^{\prime}\right)\right) \otimes\left[\mathcal{V}^{A}\right]^{\text {can }} \\
& \longleftrightarrow \mathcal{A}_{\text {sia }}^{\cdot}\left(\widetilde{\mathcal{A}}_{P, \Xi}, Z_{\Xi}^{A}\right) \otimes\left[\mathcal{V}^{A}\right]^{\text {can }}
\end{aligned}
$$

are compatible fine resolutions of

$$
\left[\mathcal{V}^{A}\right]^{\text {can }} \otimes \widetilde{\pi}^{*} \mathcal{I}_{Z_{\Xi}\left(P^{\prime}\right)} \longleftrightarrow\left[\mathcal{V}^{A}\right]^{\text {can }} \otimes \mathcal{I}_{Z_{\Xi}^{A}\left(P^{\prime}\right)} \longleftrightarrow\left[\mathcal{V}^{A}\right]^{\text {can }} .
$$

Applying the functor $R \widetilde{\pi}_{*}$ to (2.7.10.1) gives

$$
\begin{aligned}
& \tilde{\pi}_{*}\left\{\mathcal{A}_{\text {sia }}^{\bullet}\left(\widetilde{\mathcal{A}}_{P, \Xi}, Z_{\Xi}^{A}\right) \otimes\left[\mathcal{V}^{A}\right]^{\text {can }}\right\} \otimes \mathcal{I}_{Z_{\Xi}\left(P^{\prime}\right)} \\
& \longleftrightarrow\left\{\widetilde{\pi}_{*} \mathcal{A}_{\mathrm{mxa}}^{\bullet}\left(\widetilde{\mathcal{A}}_{P, \Xi} ; Z_{\Xi}^{A}, Z_{\Xi}^{A}\left(P^{\prime}\right)\right) \otimes\left[\mathcal{V}^{A}\right]^{\mathrm{can}}\right\} \\
& \hookrightarrow\left\{\widetilde{\pi}_{*} \mathcal{A}_{\text {sia }}\left(\widetilde{\mathcal{A}}_{P, \Xi}, Z_{\Xi}^{A}\right) \otimes\left[\mathcal{V}^{A}\right]^{\text {can }}\right\} .
\end{aligned}
$$

The above are taken as $\mathcal{C}_{2}$ 's in $(2.7 .8)$; for $\mathcal{C}_{1}$ 's, we take

$$
\begin{aligned}
\mathcal{A}_{\mathrm{sia}}^{\bullet}\left(\widetilde{M}_{P, \Xi}, Z_{\Xi}\right) \otimes \mathcal{H}^{\bullet}\left(\mathfrak{s}_{P}, V\right)^{\mathrm{can}} \otimes \mathcal{I}_{Z_{\Xi}\left(P^{\prime}\right)} & \longrightarrow \mathcal{A}_{\mathrm{mxa}}^{\bullet}\left(\widetilde{M}_{P, \Xi}, Z_{\Xi}\right) \otimes \mathcal{H}^{\bullet}\left(\mathfrak{s}_{P}, V\right)^{\mathrm{can}} \\
& \longrightarrow \mathcal{A}_{\mathrm{sia}}^{\bullet}\left(\widetilde{M}_{P, \Xi}, Z_{\Xi}\right) \otimes \mathcal{H}^{\bullet}\left(\mathfrak{s}_{P}, V\right)^{\mathrm{can}} .
\end{aligned}
$$

From here, it is obvious that the square in (2.7.1) commutes.

The above implies a useful fact:

Proposition 2.7.11. - The morphism

$$
H^{\bullet}\left(\widetilde{\mathcal{A}}_{P, \Xi},\left[\mathcal{V}^{A}\right]^{\text {can }}\right) \longrightarrow H^{\bullet}\left(\widetilde{\mathcal{A}}_{P, \Xi},\left[\mathcal{V}^{A}\right]^{\text {can }} \otimes \mathcal{O}_{Z_{\Xi}^{A}\left(P^{\prime}\right)}\right)
$$

is given by the direct sum of mappings

$$
H^{\bullet}\left(\widetilde{M}_{P, \Xi}, \mathcal{H}^{i}\left(\mathfrak{s}_{P}, V\right)^{\mathrm{can}}\right) \longrightarrow H^{\bullet}\left(\widetilde{M}_{P, \Xi}, \mathcal{H}^{i}\left(\mathfrak{s}_{P}, V\right)^{\mathrm{can}} \otimes \mathcal{O}_{Z_{\Xi}\left(P^{\prime}\right)}\right) .
$$


Proof. - The sheaf $\left[\mathcal{V}^{A}\right]^{\text {can }} \otimes \mathcal{O}_{Z_{\Xi}^{A}\left(P^{\prime}\right)}$ is quasi-isomorphic to the complex (2.7.11.1)

$$
\left\{\left[\mathcal{V}^{A}\right]^{\text {can }} \otimes \mathcal{I}_{Z_{\Xi}^{A}\left(P^{\prime}\right)} \longrightarrow\left[\mathcal{V}^{A}\right]^{\text {can }}\right\}[1]
$$

Then

$$
R \widetilde{\pi}_{*}\left\{\left[\mathcal{V}^{A}\right]^{\operatorname{can}} \otimes \mathcal{I}_{Z_{\Xi}^{A}\left(P^{\prime}\right)} \longrightarrow\left[\mathcal{V}^{A}\right]^{\text {can }}\right\}[1]
$$

is quasi-isomorphic to

$$
\bigoplus_{i}\left\{\mathcal{H}^{i}\left(\mathfrak{s}_{P}, V\right)^{\mathrm{can}} \otimes \mathcal{I}_{Z_{\Xi}\left(P^{\prime}\right)} \longrightarrow \mathcal{H}^{i}\left(\mathfrak{s}_{P}, V\right)^{\mathrm{can}}\right\}[1]
$$

which is in turn quasi-isomorphic to $\mathcal{H}^{\bullet}\left(\mathfrak{s}_{P}, V\right)^{\text {can }} \otimes \mathcal{O}_{Z_{\Xi}\left(P^{\prime}\right)}$. Restriction to $Z_{\Xi}^{A}\left(P^{\prime}\right)$ and $Z_{\Xi}\left(P^{\prime}\right)$ is induced by inclusion into (2.7.11.1) and (2.7.11.2). Our assertion follows. 


\section{CHAPTER 3}

\section{THE NERVE SPECTRAL SEQUENCE FOR COHERENT COHOMOLOGY}

For the remainder of the article, we fix a pure (standard) Shimura datum $(G, X)$. Thus $G$ is a reductive group over $\mathbb{Q}$; for simplicity we assume $G^{\text {ad }}$ to be $\mathbb{Q}$-simple. All mixed Shimura varieties will be realized as boundary strata of toroidal compactifications of $K_{f} \operatorname{Sh}(G, X)_{\Sigma}$. Here and in what follows the notation $K_{f}$ and $\Sigma$ are used as in $\S 1$, relative to $(G, X)$, and will be used without comment. Moreover, all toroidal data $\Sigma$ will be assumed to be full, in the sense of [HZ1,2.2.6].

\subsection{Cohomology on boundary strata of toroidal compactifications}

We refer to (0.5)-(0.7) and (1.1.9) for notation and conventions concerning rational parabolics and boundary strata. For $R \in \mathcal{P}(G), R$ subordinate to $P$, we define the $R$-stratum $Z_{\Sigma}(R)$ of $K_{f} \mathrm{Sh}(G, X)_{\Sigma}$ as in (0.6.1) and [HZ2, (1.5.2)]. We revert momentarily to the classical (non-adelic) language of $[\mathrm{HZ} 1, \S \S 1-3]$. When $R=P$ is maximal, $Z_{\Sigma}(R)$ is itself the union of irreducible divisors with normal crossings corresponding to 1 -dimensional cones in $\Sigma_{P}^{c}$ modulo conjugation by $\Gamma_{\ell, P}$ (notation as in [HZ1], esp. $\S 1.5)$. The nerve $\mathfrak{N}_{\Sigma}(R)$ of the closed covering of $Z_{\Sigma}(R)$ by its irreducible components (denoted $\mathfrak{N}\left(\mathcal{Z}_{\Sigma_{P}}\right)$ in [HZ1]) is a simplicial complex canonically isomorphic to $\Gamma_{\ell, P} \backslash \widehat{\Sigma}_{P}^{c}$ [HZ1, Lemma 3.7.2]. More generally, if $r$ is the parabolic rank of $R$, then $Z_{\Sigma}(R)$ has a closed covering by irreducible components of codimension $r$ in $K_{f} \operatorname{Sh}(G, X)_{\Sigma}$. However, the nerve $\mathfrak{N}_{\Sigma}(R)$ of this closed covering does not have a transparent description in terms of $\Sigma_{F}$. This is because, for instance, if $P \prec P_{1}$, $R=P \cap P_{1}$, and $\sigma$ (resp. $\left.\sigma^{\prime}\right) \in \Sigma_{P}$ is a two-dimensional (resp. three-dimensional) cone with one (resp. two) edge(s) in $\Sigma_{P_{1}}$ and one in $\Sigma_{P}^{c}$, then the corresponding closed toroidal stratum $\bar{Z}_{\sigma}$ (resp. $\bar{Z}_{\sigma^{\prime}}$ ) is of codimension 0 (resp. 1) in $Z_{\Sigma}(R)$ but the facets of $\sigma$ and $\sigma^{\prime}$ in $\Sigma_{P}$ have the same dimension. The constructions in [HZ2, $\left.\S 2\right]$ were designed to deal with this problem.

Let $i_{R}: Z_{\Sigma}(R) \hookrightarrow K_{f} \mathrm{Sh}(G, X)_{\Sigma}$ denote the canonical closed immersion. For $R=P$ maximal, the geometric description of $\mathfrak{N}_{\Sigma}(P)$ in $[\mathrm{HZ} 1, \S 3]$ yields a simple 
expression for the coherent cohomology $H^{\bullet}\left(Z_{\Sigma}(P), i_{P}^{*}[\mathcal{W}]^{\text {can }}\right)$, when $[\mathcal{W}]$ is an automorphic vector bundle on $\operatorname{Sh}(G, X)$ [HZ1,3.7.8, 3.13.6]. The main step is the calculation $[\mathrm{HZ1}, 3.6 .4]$ in terms of the pure Shimura variety attached to $G_{h, P}$ of the cohomology $H \bullet\left(\bar{Z}_{\sigma}, i_{\sigma}^{*}[\mathcal{W}]^{\text {can }}\right)$ where $\bar{Z}_{\sigma}$ is an individual closed stratum as above and $i_{\sigma}: \bar{Z}_{\sigma} \hookrightarrow K_{f} \operatorname{Sh}(G, X)_{\Sigma}$ is the inclusion. More precisely, the argument in [HZ1, 3.6.4] only concerns the open stratum $Z_{\sigma}$, and expresses its cohomology in terms of that of a family of automorphic vector bundles $\mathcal{V}_{\lambda(h, w)}$ on the base $M_{P}$. For $\bar{Z}_{\sigma}$ we obtain the analogous expression in terms of the canonical extensions $\left[\mathcal{V}_{\lambda(h, w)}\right]^{\text {can }}$ on the toroidal compactification $M_{P, \Sigma(P)}$, using Proposition 1.4.4 and Corollary 1.4.5; see (3.1.2), below. In particular, the calculation works in exactly the same way when $\mathfrak{N}_{\Sigma}(P)$ is replaced by $\mathfrak{N}_{\Sigma}(R)$ for general $R$.

REMARK. - Careful readers of the proofs in [HZ1] may note an apparent dependence on analytic considerations not discussed here. Specifically, the reduction of the cohomology of a boundary stratum to cohomology of reductive groups is carried out in Corollary 3.7.8 of [HZ1]. An intermediate step in the proof of this corollary is provided by Lemma 3.7 .5 of [op. cit.], which (in the present setting) calculates the coherent cohomology of $Z_{\Sigma}(P)$ in terms of the $\Gamma_{\ell, P}$-equivariant coherent cohomology of an abelian scheme, the analogue of $\operatorname{Sh}(Q / U, U \backslash X)_{\Sigma_{2}}$. In turn, the proof of Lemma 3.7.5 makes forward reference to [HZ,3.9.4], whose proof is analytic and homotopytheoretic. However, that argument was not needed; Lemma 3.7.5 is actually a simple consequence of (3.7.3), (3.7.4), and (3.7.6).

We first state the result (for general $R$ ) in the classical setting. Thus $D$ is a connected component of $X$ and $\Gamma \subset G$ is a neat arithmetic subgroup of $G(\mathbb{R})$ that fixes $X$. We retain the notation $\Gamma_{\ell, R}=\Gamma \cap G_{\ell, P}(\mathbb{Q}) \cap R(\mathbb{Q})$, when $R$ is subordinate to $P$, from [HZ2]. We fix a point $p \in D$ as in $[\mathrm{HZ} 1, \S 3.5]$, let $K_{p} \subset G(\mathbb{R})$ denote its stabilizer. We can and do always choose $p$ so that $K_{p} \cap P(\mathbb{R})$ contains a maximal connected compact subgroup of $P(\mathbb{R})$ for every standard parabolic $P$. As in [HZ1], we restrict attention to fully decomposed automorphic vector bundles; i.e., those associated to representations of $K_{p}$ ([HZ1, Definition 3.1.2]).

We let $M_{P}$ denote the (connected) pure Shimura variety associated to $P$. Similarly, we let $W^{P, p}$ denote the subset of the Weyl group of $K_{p}$ from [HZ1, $\left.\S 3.6\right]$ (see (0.5)). Recall that this is the set of Kostant representatives for the maximal parabolic denoted $Q^{P, p} \subset K_{p}$ with Levi subgroup $K_{p}^{(2)}$ and unipotent radical $S_{p}$. The Lie algebras are denoted by lower case Gothic characters, as usual. Recall further [HZ1, (1.8.3), (1.8.6)] that the Cayley transform induces isomorphisms

$$
K_{p}^{P,(2)} \stackrel{\sim}{\longrightarrow} K_{h, P} \times G_{\ell, P} ; \quad \mathfrak{s}_{p, P} \stackrel{\sim}{\longrightarrow} \mathfrak{v}_{p}^{-} .
$$

Here $K_{h, P} \subset G_{h, P}$ is the maximal compact subgroup associated to $p$. More generally, if $R$ is subordinate to the maximal parabolic $P$, we let $Q^{R, p} \subset Q^{P, p}$ be the parabolic with Levi factor isomorphic via Cayley transform to $K_{h, P} \times G_{\ell, R}$ and unipotent radical 
$S_{p, R} \supset S_{p, P}$ and let $W^{R, p}$ be the corresponding set of Kostant representatives in the Weyl group of $K_{p}$.

Proposition 3.1.1. - Let $[\mathcal{W}]$ be an irreducible automorphic vector bundle on $\operatorname{Sh}(G, X)$ and let $R$ be a proper $\mathbb{Q}$-parabolic subgroup of $G$, subordinate to the maximal parabolic $P$. Then

(i) For each $b \geqslant 0$, the assignment

$$
\sigma \longmapsto H^{b}\left(\bar{Z}_{\sigma}, i_{\sigma}^{*}[\mathcal{W}]^{\text {can }}\right)
$$

defines a locally constant sheaf $\boldsymbol{L}^{b}(\cdot, \mathcal{W})$ on the simplicial complex $\mathfrak{N}_{\Sigma}(R)$, and an associated spectral sequence

$$
E_{1}^{a, b}=H^{a}\left(\mathfrak{N}_{\Sigma}(R), \boldsymbol{L}^{b}(\cdot, \mathcal{W})\right) \Longrightarrow H^{a+b}\left(Z_{\Sigma}(R), i_{R}^{*}[\mathcal{W}]^{\text {can }}\right) .
$$

(ii) Over the universal cover of $\mathfrak{N}_{\Sigma}(R), \boldsymbol{L}^{b}(\bullet, \mathcal{W})$ is isomorphic to

$$
\bigoplus_{w \in W^{P, p}} H^{b-\ell(w)}\left(M_{P, \Sigma(P)},\left[\mathcal{V}_{\lambda(h, w)}\right]^{\mathrm{can}}\right) \otimes V_{\lambda(\ell, w)} .
$$

Here $M_{P, \Sigma(P)}$ is any admissible toroidal compactification of $M_{P}, \ell(w)$ is the length of $w$ and $\mathcal{V}_{\lambda(h, w)}$ and $V_{\lambda(\ell, w)}$ are defined (relative to $\left.[\mathcal{W}]\right)$ as in $[\mathrm{HZ1},(3.6 .1)]$.

(iii) The spectral sequence in (i) degenerates at $E_{1}$, and is naturally split.

\section{REMARKS 3.1.2}

(i) The hypothesis that $[\mathcal{W}]$ be irreducible is made in order to obtain the simple decomposition (3.1.1.3). This hypothesis was inadvertently omitted from the discussion in $[\mathrm{HZ} 1, \S 3]$.

(ii) For $R=P$ maximal, the above is a strengthening of (3.7.3), (3.7.4) and (3.7.7) of [HZ1], which pertain to the (open) $P$-stratum. (Note the misprint in [loc. cit], where the spectral sequence was labelled $E_{2}$ rather than $E_{1}$.) Here, we extend those results in two ways: we are working with the closed $R$-stratum $Z_{\Sigma}(R)$, and this is for arbitrary $R$. Correspondingly, the above formulas involve canonical extension to some toroidal compactification $M_{P, \Sigma(P)}$.

Proof of (3.1.1). - We adapt the line of reasoning in [HZ1,§3]. By [P, Corollary 7.17], every closed stratum $\bar{Z}_{\sigma} \subset Z_{\Sigma}(R)$ (whose interior was constructed from a cone in $\Sigma_{P}$ ) can be realized as a toroidal compactification of a (connected) mixed Shimura variety of the form ${ }_{K_{f}} \operatorname{Sh}(Q, X)_{\Sigma}$, of the sort considered in (1.4). For appropriately chosen $\Sigma$, we get a morphism

$$
\pi_{\sigma}=\pi_{2, \sigma} \circ \pi_{1, \sigma}: \bar{Z}_{\sigma} \longrightarrow M_{P, \Sigma(P)}
$$

as in (1.4.2). To obtain the formulas in (3.1.1), we incorporate growth conditions at the toroidal boundary, replacing Corollary 3.6.3 of [loc. cit.] by Proposition 1.4.4 
here. Then, Corollary 1.4.5 (the generalization of the Leray spectral sequences of [HZ1, 3.5.10]) reads:

$$
E_{2}^{p, q}=H^{p}\left(M_{P, \Sigma(P)},\left(R^{q} \Phi_{\sigma} i_{\sigma}^{*}[\mathcal{W}]\right)^{\text {can }}\right) \Longrightarrow H^{p+q}\left(\bar{Z}_{\sigma}, i_{\sigma}^{*}[\mathcal{W}]^{\text {can }}\right)=\boldsymbol{L}^{p+q}(\sigma, \mathcal{W}) .
$$

Moreover, as noted in Remark (1.2.16.3), $i_{\sigma}^{*}[\mathcal{W}]^{\text {can }}$ satisfies a Kostant condition in this case. Hence this Leray spectral sequence degenerates at $E_{2}$ and the grading induced by the $E_{2}$-term splits canonically:

$$
\begin{aligned}
\boldsymbol{L}^{b}(\sigma, \mathcal{W}) & =\bigoplus_{q} H^{b-q}\left(M_{P, \Sigma(P)},\left(R^{q} \Phi_{\sigma} i_{\sigma}^{*}[\mathcal{W}]\right)^{\mathrm{can}}\right) \\
& =\bigoplus_{w \in W^{P, p}} H^{b-\ell(w)}\left(M_{P, \Sigma(P)},\left[\mathcal{V}_{\lambda(h, w)}\right]^{\mathrm{can}}\right) \otimes V_{\lambda(\ell, w)} .
\end{aligned}
$$

The second equality, the decomposition of the higher direct image as a sum over Kostant representatives, is (the version with growth conditions of) [HZ1,Corollary 3.6.4].

Note that the final expression is independent of $\sigma$. As in [HZ1, § 3.6], this expression is compatible with all restriction maps for the simplicial complex $\mathfrak{N}_{\Sigma}(R)$ [loc. cit., 3.6.4] and with the action of the fundamental group of $\mathfrak{N}_{\Sigma}(R)$ ([loc. cit, 3.6.3]; see below). This proves (i) and (ii), and implies that the $E_{1}$-term in (3.1.1.2) is canonically isomorphic to

$$
\bigoplus_{w \in W^{P, p}} H^{a}\left(\pi_{1}\left(\mathfrak{N}_{\Sigma}(R)\right), V_{\lambda(\ell, w)}\right) \otimes H^{b-\ell(w)}\left(M_{P, \Sigma(P)},\left[\mathcal{V}_{\lambda(h, w)}\right]^{\mathrm{can}}\right) .
$$

(The fundamental group of $\mathfrak{N}_{\Sigma}(R)$ is identified with $\Gamma_{\ell, R}$ in Proposition 3.1.3, below.) Let $\widetilde{\boldsymbol{V}}_{\lambda(\ell, w)}$ denote the local system on $\mathfrak{N}_{\Sigma}(R)$ associated to the representation of the fundamental group on $V_{\lambda(\ell, w)}$. Thus (iii) follows from the Künneth formula, or more precisely from the universal coefficient theorem, applied to the sum over $w \in W^{P, p}$ of the local systems

$$
\widetilde{\boldsymbol{V}}_{\lambda(\ell, w)} \otimes H^{b-\ell(w)}\left(M_{P, \Sigma(P)},\left[\mathcal{V}_{\lambda(h, w)}\right]^{\mathrm{can}}\right)
$$

on $\mathfrak{N}_{\Sigma}(R)$, as in the last part of the proof of Proposition 3.7.7 of [HZ1].

In $\S 2$ of [HZ2] the nerves $\mathfrak{N}_{\Sigma}(R)$ are identified for general $R$, up to homotopy type. Let $X\left(\Gamma_{\ell, P}\right)$ be the locally symmetric space associated to the arithmetic subgroup $\Gamma_{\ell, P}\left(\right.$ denoted $X\left(\Gamma_{\ell}\right)$ in $\left.[\mathrm{HZ1}, 2.2 .10]\right)$. Let $X\left(\Gamma_{\ell, P}\right) \hookrightarrow \bar{X}\left(\Gamma_{\ell, P}\right)$ denote the BorelSerre compactification. The result is the following:

Proposition 3.1.3. - Let $R$ be a proper $\mathbb{Q}$-parabolic subgroup of $G$, subordinate to the maximal parabolic $P$. As above, let $R_{\ell, P}$ denote the corresponding $\mathbb{Q}$-parabolic subgroup of $G_{\ell, P}$. Then

(i) The nerve $\mathfrak{N}_{\Sigma}(R)$ is homotopy equivalent to the $R_{\ell, P}$-stratum $\overline{e^{\prime}\left(R_{\ell, P}\right)} \subset$ $\bar{X}\left(\Gamma_{\ell, P}\right)$.

(ii) Let $R \subset R^{\prime}$ be two $\mathbb{Q}$-parabolic subgroups of $G$ subordinate to P. Under the homotopy equivalences in (i), the natural inclusion $\mathfrak{N}_{\Sigma}(R) \subset \mathfrak{N}_{\Sigma}\left(R^{\prime}\right)$ corresponds to the natural inclusion $\overline{e^{\prime}\left(R_{\ell, P}\right)} \subset \overline{e^{\prime}\left(R_{\ell, P}^{\prime}\right)}$. 
This is essentially contained in Proposition 2.6.4 of [HZ2]; the translation into the present language is provided by Corollaries 2.5.9 and 2.5.10 of [loc. cit.].

The fundamental group of $\overline{e^{\prime}\left(R_{\ell, P}\right)}$ is just $\Gamma_{\ell, R}$. Let $\tilde{\boldsymbol{V}}_{\lambda(\ell, w)}$ denote the local system on $\bar{X}\left(\Gamma_{\ell, P}\right)$ associated to the representation $\lambda(\ell, w)$, as in the proof of (3.1.1) (cf. the statement of Corollary 3.7.8 of [HZ1]). Just as in the case of maximal strata (cf. [HZ1, Corollary 3.7.8]), Proposition 3.1.3 and (3.1.2) allow us to identify

$$
\begin{aligned}
& H^{a}\left(\mathfrak{N}_{\Sigma}(R), \boldsymbol{L}^{b}(\bullet, \mathcal{W})\right) \simeq \oplus_{w \in W^{P, p}} H^{a}\left(\Gamma_{\ell, R}, H^{b-\ell(w)}\left(M_{P, \Sigma(P)},\left[\mathcal{V}_{\lambda(h, w)}\right]^{\mathrm{can}}\right) \otimes V_{\lambda(\ell, w)}\right) \\
& \simeq \oplus_{w \in W^{P, p}} H^{b-\ell(w)}\left(M_{P, \Sigma(P)},\left[\mathcal{V}_{\lambda(h, w)}\right]^{\mathrm{can}}\right) \otimes H^{a}\left(\Gamma_{\ell, R}, V_{\lambda(\ell, w)}\right) \\
&(3.1 .4) \simeq \oplus_{w \in W^{P, p}} H^{b-\ell(w)}\left(M_{P, \Sigma(P)},\left[\mathcal{V}_{\lambda(h, w)}\right]^{\mathrm{can}}\right) \otimes H^{a}\left(\overline{e^{\prime}\left(R_{\ell, P}\right)}, i_{R_{\ell, P}}^{*} \widetilde{\boldsymbol{V}}_{\lambda(\ell, w)}\right)
\end{aligned}
$$

where the second isomorphism expresses the fact that $\Gamma_{\ell, R}$ acts trivially on the first factor, and in the third isomorphism $i_{R_{\ell, P}}: \overline{e^{\prime}\left(R_{\ell, P}\right)} \hookrightarrow \bar{X}\left(\Gamma_{\ell, P}\right)$ is the natural inclusion.

On the other hand, the calculation of $H^{\bullet}\left(\overline{e^{\prime}\left(R_{\ell, P}\right)}, i_{R_{\ell, P}}^{*} \tilde{\boldsymbol{V}}_{\lambda(\ell, w)}\right)$ is standard. Let $W_{\ell, P}$ denote the relative Weyl group (over $\mathbb{Q}$ ) of $G_{\ell, P}$, and let $W_{\ell}^{R} \subset W_{\ell, P}$ denote the subset of Kostant representatives relative to $R_{\ell, P}$ (cf. Notation). Identify $G_{\ell, R}$ with a Levi quotient of $R_{\ell, P}$ and let $\Gamma_{\ell, R}^{\text {red }}$ denote the image of $\Gamma_{\ell, R}$ in $G_{\ell, R}(\mathbb{Q})$; let $X\left(\Gamma_{\ell, R}^{\text {red }}\right)$ be the corresponding locally symmetric space, the quotient by $\Gamma_{\ell, R}^{\mathrm{red}}$ of the symmetric space attached to $G_{\ell, R}$. Let $\mu(\ell, w)$ denote the highest weight of $\lambda(\ell, w)$ relative to the choice of positive roots for $G_{\ell, P}$ made in [HZ1,3.6]. Then [Ha]

$$
H^{a}\left(\overline{e^{\prime}\left(R_{\ell, P}\right)}, i_{R_{\ell, P}}^{*} \tilde{\boldsymbol{V}}_{\lambda(\ell, w)}\right) \stackrel{\sim}{\longrightarrow} \bigoplus_{\omega \in W_{\ell}^{R}} H^{a-\ell(\omega)}\left(X\left(\Gamma_{\ell, R}^{\mathrm{red}}\right), \tilde{\boldsymbol{V}}_{\lambda(\ell, w ; \omega)}\right)
$$

Here $\widetilde{\boldsymbol{V}}_{\lambda(\ell, w ; \omega)}$ is the local system on $X\left(\Gamma_{\ell, R}^{\mathrm{red}}\right)$ associated to the representation $\lambda(\ell, w ; \omega)$ of $G_{\ell, R}$, and this in turn is the representation with highest weight $\omega\left(\mu(\ell, w)+\rho_{\ell}\right)-\rho_{\ell}$, where $\rho_{\ell}$ is the half-sum of positive roots of $G_{\ell, P}$.

The following easy lemma has already been used several times in Chapter 1, and will be used repeatedly in what follows. Presumably it is well known but we did not find a reference.

Lemma 3.1.6. - Let $H$ be a quasisplit reductive group over the field $k$, with Borel subgroup $B$, and let $P_{1} \supset P_{2}$ be a pair of standard $k$-parabolic subgroups of $G$, with Levi decompositions $P_{1}=L_{1} \cdot U_{1}, P_{2}=L_{2} \cdot U_{2}, L_{1} \supset L_{2}$. Let $Q=P_{2} \cap L_{1}$. Let $T \subset B$ be a maximal $k$-split torus and let $W=W(H, T)$ and $W_{1}=W\left(L_{1}, T\right)$ be the relative Weyl groups. Let $W^{i} \subset W$ be the sets of Kostant representatives for $P_{i}$, $i=1,2$; let $W^{Q} \subset W_{1}$ be the set of Kostant representatives for $Q$. (In both cases the Kostant representatives are defined relative to the ordering determined by B.) Under the natural identification of $W_{1}$ with a subgroup of $W$, we then have

$$
W^{2}=\left\{\omega \cdot w \mid \omega \in W^{Q}, w \in W^{1}\right\} .
$$


Moreover, with the above notation, $\ell_{W}(\omega \cdot w)=\ell_{W}(w)+\ell_{W_{1}}(\omega)$, where $\ell_{W}$ and $\ell_{W_{1}}$ are the length functions on $W$ and $W_{1}$, respectively.

Proof. - The map

$$
W^{Q} \times W^{1} \longrightarrow W ; \quad \omega \times w \longmapsto \omega \cdot w
$$

is injective, since $W^{1}$ is a set of right coset representatives $\bmod W_{1}$. If the image is contained in $W^{2}$ then it must equal $W^{2}$, since the two sets have the same cardinality. Let $w^{\prime}=\omega \cdot w$ as above, and suppose $\alpha$ is a positive root in $L_{2}$. Then $\omega^{-1}(\alpha)$ is a positive root in $L_{1}$, by definition of $W^{Q}$, hence

$$
w^{\prime,-1}(\alpha)=w^{-1} \omega^{-1}(\alpha)
$$

is a positive root in $H$, by definition of $W^{1}$. Since $\ell\left(w^{\prime}\right)=\ell\left(w^{\prime,-1}\right)$, the assertion about the lengths follows from the definitions by counting the positive roots made negative by $w^{\prime,-1}=w^{-1} \omega^{-1}$, bearing in mind that $W_{1} \subset L_{1}$ fixes the set of positive roots of the unipotent radical of $P_{1}$.

Applying Lemma 3.1.6, with $P_{1}=Q^{P, p}, P_{2}=Q^{R, p}$, and thus $Q \simeq R_{\ell, P}$, to (3.1.4) and (3.1.5), we thus find for $(3.1 .1)(\mathrm{i})$

$$
E_{2}^{a, b} \simeq \bigoplus_{w=w_{1} \cdot w_{2} \in W^{R, p}} H^{b-\ell\left(w_{2}\right)}\left(M_{P, \Sigma(P)},\left[\mathcal{V}_{\lambda(h, w)}\right]^{\text {can }}\right) \otimes H^{a-\ell\left(w_{1}\right)}\left(X\left(\Gamma_{\ell, R}^{\mathrm{red}}\right), \widetilde{\boldsymbol{V}}_{\lambda(\ell, w)}\right)
$$

Here $w \in W^{R, p}$ is factored as in Lemma 3.1.6 as $w_{1} \cdot w_{2}$ with $w_{1} \in W_{\ell}^{R}$ and $w_{2} \in W^{P, p}$; $\ell\left(w_{i}\right)$ is given by the appropriate length function; and $\lambda(\ell, w)=\lambda\left(\ell, w_{2} ; w_{1}\right)$ in the previous notation. Moreover, we have used Kostant's theorem to write

$$
H^{i}\left(\mathfrak{s}_{p, R}, V_{\lambda}\right)=\bigoplus_{w \in W^{R, p} ; \ell(w)=i} V_{\lambda(h, w)} \otimes V_{\lambda(\ell, w)}
$$

where $V_{\lambda(h, w)}=V_{\lambda\left(h, w_{2}\right)}$ is a representation of $K_{h, P}$ and $V_{\lambda(\ell, w)}$ is a representation of $G_{\ell, R}$.

Combining this with (3.1.1)(iii), we obtain the following formula:

Proposition 3.1.8. - Let $[\mathcal{W}]$ be an irreducible automorphic vector bundle on $\operatorname{Sh}(G, X)$, and let $R$ be a proper $\mathbb{Q}$-parabolic subgroup of $G$, subordinate to the maximal parabolic $P$. Then

$$
\begin{aligned}
& H^{k}\left(Z_{\Sigma}(R), i_{R}^{*}[\mathcal{W}]^{\text {can }}\right) \\
& \quad \simeq \bigoplus_{a+b=k} \bigoplus_{w \in W^{R, p}} H^{b-\ell(w)}\left(M_{P, \Sigma(P)},\left[\mathcal{V}_{\lambda(h, w)}\right]^{\text {can }}\right) \otimes H^{a}\left(X\left(\Gamma_{\ell, R}^{\mathrm{red}}\right), \widetilde{\boldsymbol{V}}_{\lambda(\ell, w)}\right) .
\end{aligned}
$$

REMARK 3.1.9

(i) In the setting of Lemma 3.1.6, let $\mathfrak{u}_{i}$ denote the Lie algebra of $U_{i}$ for $i=1,2$ and $\mathfrak{u}_{Q}$ the Lie algebra of the unipotent radical of $Q$. Then $\mathfrak{u}_{2}=\mathfrak{u}_{1} \oplus \mathfrak{u}_{Q}$. If follows from Lemma (3.1.6) that the spectral sequence

$$
E_{2}^{p, q}=H^{q}\left(\mathfrak{u}_{Q}, H^{p}\left(\mathfrak{u}_{1}, V\right)\right) \Longrightarrow H^{p+q}\left(\mathfrak{u}_{2}, V\right) .
$$


degenerates at $E_{2}$.

(ii) Here and in what follows, the passage from the sum over $W^{P, p}$ to a sum over $W^{R, p}$ should be regarded as a purely topological operation. All the arithmetic information is carried by the $G_{h, P}$-factor, and is independent of the parabolic subgroup $R$ subordinate to $P$. In particular:

(a) The isomorphism (3.1.5) is rational over the field of coefficients of the automorphic vector bundle $[\mathcal{W}]$. This is because the spectral sequence in (i) above, with $Q=R_{\ell, P}$, is purely algebraic and $\mathfrak{u}_{Q}$ is a $\mathbb{Q}$-rational nilpotent Lie subalgebra of $\mathfrak{g}$. In particular, the adelic reformulation (3.2.9) of the calculations of this section is compatible with the canonical models of $\operatorname{Sh}(G, X)$ and $\operatorname{Sh}\left(G_{h, P}, X(P)\right)$.

(b) Similarly, for the Hodge-theoretic applications in $\S 4$, it makes no difference whether the cohomology of the $R$-stratum is written as a sum indexed by $W^{P, p}$ or by $W^{R, p}$. (Compare (4.2.27) and (4.2.29)). Again, this is because the contribution of the $G_{\ell, R}$-factor is purely topological.

\subsection{The nerve spectral sequence for boundary cohomology}

The spectral sequence in question is the one associated to the closed covering $\left\{Z_{\Sigma}(P): P\right.$ maximal $\}$ of $Z_{\Sigma}$. In classical language, the $E_{1}$-term is

$$
\begin{gathered}
E_{1}^{r, s}=\bigoplus_{r(R)=r+1} E_{1}^{r, s}(R) \Longrightarrow H^{r+s}\left(Z_{\Sigma}, i^{*}[\mathcal{W}]^{\text {can }}\right), \quad \text { where } \\
E_{1}^{r, s}(R)=H^{s}\left(Z_{\Sigma}(R), i_{R}^{*}[\mathcal{W}]^{\text {can }}\right) .
\end{gathered}
$$

Using the description of the right-hand side of (3.2.2) given in (3.1.8), we can write the above as

$$
E_{1}^{r, s}(R)=\bigoplus_{a+b=s} \bigoplus_{w \in W^{R, p}} H^{b-\ell(w)}\left(M_{P, \Sigma(P)},\left[\mathcal{V}_{\lambda(h, w)}\right]^{\mathrm{can}}\right) \otimes H^{a}\left(X\left(\Gamma_{\ell, R}^{\mathrm{red}}\right), \widetilde{\boldsymbol{V}}_{\lambda(\ell, w)}\right) .
$$

For $R$ of parabolic rank $r+1$ and $R^{\prime} \subset R$ of parabolic corank 1 , we will see that the nature of the contribution to $d_{1}$,

$$
d_{1}\left(R, R^{\prime}\right): E_{1}^{r, s}(R) \longrightarrow E_{1}^{r+1, s}\left(R^{\prime}\right),
$$

depends on whether or not $\Pi\left(R^{\prime}\right)=\Pi(R)$ (in the notation of $(0.7)$ ).

We set up the adelic version of the above. We drop the level subgroup $K_{f}$ from the notation and write $\mathrm{Sh}_{\Sigma}$ for $\operatorname{Sh}(G, X)_{\Sigma}, \partial \mathrm{Sh}_{\Sigma}$ for its (toroidal) boundary, $\operatorname{Sh}_{\Sigma}^{R(*)}$ for its $R(*)$-stratum, and $\overline{\mathrm{Sh}}_{\Sigma}^{R(*)}$ for the Zariski closure of $\mathrm{Sh}_{\Sigma}^{R(*)}$ in $\partial \mathrm{Sh}_{\Sigma}$. As in [HZ1, 5.3], the $\overline{\mathrm{Sh}}_{\Sigma}^{P(*)}$, as $P$ runs through the maximal admissible parabolics, form a closed cover of $\partial \mathrm{Sh}_{\Sigma}$. Taking the nerve of this closed cover thus yields a spectral sequence for the cohomology of $\partial \mathrm{Sh}_{\Sigma}$ with coefficients in a canonically extended automorphic vector 
bundle. For simplicity we describe this spectral sequence when $G^{\text {ad }}$ is assumed $\mathbb{Q}$ simple. Let $i_{R}: \overline{\mathrm{Sh}}_{\Sigma}^{R(*)} \hookrightarrow \mathrm{Sh}_{\Sigma}$ denote the corresponding closed embedding, and let $r(R)$ denote the parabolic rank of $R$. Taking the limit over neat open compact subgroups and families of fans, we obtain the nerve spectral sequence in the following form:

$$
E_{1}^{r, s}=\bigoplus_{r(R)=r+1} \underset{\overline{l i m}_{f}, \Sigma}{\longrightarrow} H^{s}\left(\overline{\operatorname{Sh}}_{\Sigma}^{R(*)}, i_{R}^{*}\left([\mathcal{W}]^{\text {can }}\right)\right) \Longrightarrow H^{r+s}([\mathcal{W}](\infty)) .
$$

Here $H \bullet([\mathcal{W}](\infty))$ denotes $\lim _{K_{f}, \Sigma} H \bullet\left(\partial \mathrm{Sh}_{\Sigma},[\mathcal{W}]^{\text {can }} \otimes \mathcal{O}_{\partial \mathrm{Sh}_{\Sigma}}\right)$, as in [HZ1].

The individual summands on the left-hand side of (3.2.4) can be written as induced representations, just as in [HZ1]. It is most convenient to express each term as a tensor product of the coherent cohomology of a (pure) Shimura variety by the cohomology of a locally symmetric space. Let $R$ be any rational parabolic, subordinate to the maximal rational parabolic $P$, and define $G_{\ell, R}$ as before. We define the adelic locally symmetric space

$$
X\left(G_{\ell, R}\right)=\lim _{\longleftarrow} G_{\ell, R}(\mathbb{Q}) \backslash G_{\ell, R}(\boldsymbol{A}) /\left(K_{\ell, R} \cdot Z_{\ell, R}\right)(\mathbb{R}) \cdot K_{\ell, R, f},
$$

where $Z_{\ell, R}$ is the center of $G_{\ell, R}, K_{\ell, R}=K_{p} \cap G_{\ell, R}(\mathbb{R})$, and $K_{\ell, R, f}$ runs through compact open subgroups of $G_{\ell, R}\left(\boldsymbol{A}_{f}\right)$. Let $L_{R}$ be a (standard) Levi subgroup of $R$. We also let $\operatorname{Sh}\left(G_{h, P}, X(P)\right)$ denote the Shimura variety attached to $G_{h, P}$. For brevity we write $\operatorname{Sh}(R)=\operatorname{Sh}\left(G_{h, P}, X(P)\right)$ when $R$ is subordinate to $P$. For each $w \in W^{R, p}$ we have the automorphic vector bundle $\left[\mathcal{V}_{\lambda(h, w)}\right]$ over $\operatorname{Sh}(R)$, as above, and therefore we can define the cohomology of its canonical extensions in the adelic limit

$$
\widetilde{H}^{\bullet}\left(\left[\mathcal{V}_{\lambda(h, w)}\right]\right)
$$

(notation as in [H4] and $[\mathrm{HZ} 1, \S 4])$. We define

$$
\mathcal{H}^{\bullet}(w)=\widetilde{H}^{\bullet}\left(\left[\mathcal{V}_{\lambda(h, w)}\right]\right) \otimes H^{\bullet}\left(X\left(G_{\ell, R}\right), \tilde{\boldsymbol{V}}_{\lambda(\ell, w)}\right)[-\ell(w)]
$$

by analogy with $[\mathrm{HZ} 1,(4.1 .10)]$

Parametrization of connected components introduces annoying complications, as in $[\mathrm{HZ1}, 4.1 .12]$. We let

$$
\Delta_{1, R}=\operatorname{Ker}: G_{h}\left(\boldsymbol{A}_{f}\right) \times G_{\ell, R}\left(\boldsymbol{A}_{f}\right) \longrightarrow G_{h}\left(\boldsymbol{A}_{f}\right) \cdot G_{\ell, R}\left(\boldsymbol{A}_{f}\right)
$$

(the product viewed as a subgroup of $R\left(\boldsymbol{A}_{f}\right)$ ) and let

$$
\Delta_{0, R}=L_{R}(\mathbb{Q})^{+} /\left(G_{h}(\mathbb{Q})^{+} \cdot G_{\ell, R}(\mathbb{Q})^{+}\right) ; \quad \Delta_{R}=\Delta_{0, R} \times \Delta_{1, R}
$$

(For the relation between (3.2.7) and connected components of the $R$ stratum when $R$ is maximal, see [HZ1, (4.1.10,4.1.11)]. The analogous relation holds for general $R$.) Then $G_{h}\left(\boldsymbol{A}_{f}\right) \times G_{\ell, R}\left(\boldsymbol{A}_{f}\right)$ acts on $\mathcal{H}^{\bullet}(w)$, and therefore so does its subgroup $\Delta_{1, R}$. Moreover $\Delta_{0, R}$ acts on $\operatorname{Sh}(R) \times X\left(G_{\ell, R}\right)$ by the analogue of $[\mathrm{HZ1},(4.1 .11)]$. We define

$$
I^{R}\left\{\mathcal{H}^{\bullet}(w)\right\}=\operatorname{Ind}_{G_{h}\left(\boldsymbol{A}_{f}\right) \cdot G_{\ell, R}\left(\boldsymbol{A}_{f}\right) \rtimes \Delta_{0, R}}^{L_{R}\left(\boldsymbol{A}_{f}\right)}\left\{\mathcal{H}^{\bullet}(w)^{\Delta_{1, R}}\right\},
$$

just as in $[\mathrm{HZ1},(4.1 .13)]$. Then we have 
Corollary 3.2 .9

(i) In the spectral sequence (3.2.4), there is a natural decomposition $E_{1}^{r, s}=$ $\bigoplus_{r(R)=r+1} E_{1}^{r, s}(R)$, where

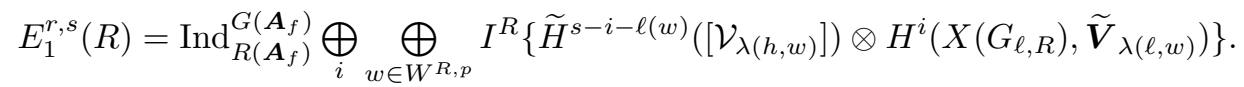

(ii) Moreover, suppose $R^{\prime} \subset R$ are two $\mathbb{Q}$-parabolic subgroups of $G$, both subordinate to $P$, with $r\left(R^{\prime}\right)=r+2, r(R)=r+1$. Let $Q^{\prime}=R_{\ell, P}^{\prime}, Q\left(R^{\prime}\right)=Q^{\prime} \cap G_{\ell, R}$. Let $d_{R^{\prime}, R}$ denote the restriction $E_{1}^{r, s}(R)$ of the nerve spectral sequence differential $d_{1}: E_{1}^{r, s} \rightarrow E_{1}^{r+1, s}$, followed by projection to $E_{1}^{r+1, s}\left(R^{\prime}\right)$. Then, with respect to the above formula, $d_{R^{\prime}, R}$ is induced by the identity on the holomorphic component tensored with the boundary map induced by the inclusion of the boundary stratum $\overline{e^{\prime}\left(Q\left(R^{\prime}\right)\right)}$ in the Borel-Serre compactification of $X\left(G_{\ell, R}\right)$.

Proof. - The first statement generalizes Corollary 4.1.14 of [HZ1], and is proved in the same way. The second statement follows immediately from (ii) of Proposition 3.1.3 (as such, it is deduced from the same for (3.2.3)).

\subsection{Differentials in the nerve spectral sequence}

Corollary 3.2.9 completes the verification of [HZ1, (5.3.12.4)], and computes half of the differentials $d_{1}$ in the nerve spectral sequence in terms of the cohomology of locally symmetric spaces attached to reductive groups. The present section will begin the computation of the remaining half: the $d_{R^{\prime}, R}$ for $r\left(R^{\prime}\right)=r(R)+1$ with $R$ and $R^{\prime}$ subordinate to distinct maximal parabolics, say $P$ and $P^{\prime}$, respectively. Let $P\left(P^{\prime}\right)=$ $P^{\prime} \cap G_{h, P}$, as in (1.4.6). In terms of the expression for $E_{1}^{r, s}$ in Corollary 3.2.9, $d_{R^{\prime}, R}$ will be expressed in terms of the restriction map from the first (holomorphic) tensor factor to the boundary stratum corresponding to $P\left(P^{\prime}\right)$. In other words, $d_{R^{\prime}, R}$, which is given a priori by a restriction map on the coherent cohomology of mixed Shimura varieties - more precisely, the cohomology of simplicial schemes whose components are all toroidal compactifications of mixed Shimura varieties - can be expressed as a restriction map whose source is the coherent cohomology of a toroidally compactified pure Shimura variety. This is the opposite of the story in (ii) in Corollary 3.2.9, and will be proved in (3.4) below.

As usual, we will verify this at the level of connected Shimura varieties at finite level, the extension to the adelic setting being routine. Thus we have isomorphisms

$$
E_{1}^{r, s}(R) \stackrel{\sim}{\longrightarrow} \underset{w \in W^{R, p}}{\longrightarrow} \bigoplus_{i} H^{s-i-\ell(w)}\left(M_{P, \Sigma(P)},\left[\mathcal{V}_{\lambda(h, w)}\right]^{\mathrm{can}}\right) \otimes H^{i}\left(X\left(\Gamma_{\ell, R}^{\mathrm{red}}\right), \widetilde{\boldsymbol{V}}_{\lambda(\ell, w)}\right)
$$


and

$$
\begin{aligned}
& E_{1}^{r+1, s}\left(R^{\prime}\right) \stackrel{\sim}{\longrightarrow} \\
& \bigoplus_{w^{\prime} \in W^{R^{\prime}, p}} \bigoplus_{j} H^{s-j-\ell\left(w^{\prime}\right)}\left(M_{P^{\prime}, \Sigma\left(P^{\prime}\right)},\left[\mathcal{V}_{\lambda\left(h, w^{\prime}\right)}\right]^{\mathrm{can}}\right) \otimes H^{j}\left(X\left(\Gamma_{\ell, R^{\prime}}^{\mathrm{red}}\right), \tilde{\boldsymbol{V}}_{\lambda\left(\ell, w^{\prime}\right)}\right)
\end{aligned}
$$

On the other hand, letting $[\mathcal{B}]$ denote any automorphic vector bundle on $M_{P, \Sigma(P)}$, we have by (3.1.8) applied to $G_{h, P}$

$$
\begin{aligned}
& H^{t}\left(\overline{\operatorname{Sh}_{\Sigma}^{P\left(P^{\prime}\right)(*)}}, i_{P\left(P^{\prime}\right)}^{*}[\mathcal{B}]^{\text {can }}\right) \stackrel{\sim}{\longrightarrow} \\
& \quad \bigoplus_{\omega \in W^{P\left(P^{\prime}\right), p^{\prime}}} H^{t-k-\ell(\omega)}\left(M_{P^{\prime}, \Sigma\left(P^{\prime}\right)},\left[\mathcal{B}_{\lambda(h, \omega)}\right]^{\text {can }}\right) \otimes H^{k}\left(X\left(\Gamma_{\ell, P\left(P^{\prime}\right)}\right), \widetilde{B}_{\lambda(\ell, \omega)}\right)
\end{aligned}
$$

Here $\left.\left[\mathcal{B}_{\lambda(h, \omega}\right)\right]$ and $\widetilde{\boldsymbol{B}}_{\lambda(\ell, \omega)}$ bear the same relation to $[\mathcal{B}]$ (for the boundary component $M_{P^{\prime}, \Sigma\left(P^{\prime}\right)}$ of $\left.M_{P, \Sigma(P)}\right)$ as $\left[\mathcal{V}_{\lambda(h, \omega}\right)$ and $V_{\lambda(\ell, \omega)}$ bear to $[\mathcal{W}]$ (for the boundary component $M_{P, \Sigma(P)}$ of $\left.\operatorname{Sh}(G, X)\right)$. Finally, $p^{\prime}$ is the fixed point of the subgroup $K_{p} \cap G_{h, P}$. Bear in mind that

$$
G_{\ell, R^{\prime}}=G_{\ell, R} \cdot G_{\ell, P\left(P^{\prime}\right)},
$$

where $G_{\ell, P\left(P^{\prime}\right)} \subset G_{h, P}$ is the $G_{\ell}$-factor of the maximal parabolic $P\left(P^{\prime}\right) \subset G_{h, P}$. Then for sufficiently small $\Gamma$, we have

$$
\Gamma_{\ell, R^{\prime}}=\Gamma_{\ell, R} \times \Gamma_{\ell, P\left(P^{\prime}\right)} ; \quad \Gamma_{\ell, R^{\prime}}^{\mathrm{red}}=\Gamma_{\ell, R}^{\mathrm{red}} \times \Gamma_{\ell, P\left(P^{\prime}\right)} .
$$

Moreover, Lemma 3.1.6 allows us to write

$$
W^{R^{\prime}, p}=W^{P\left(P^{\prime}\right), p^{\prime}} \cdot W^{R, p} .
$$

Say $w^{\prime} \in W^{R^{\prime}, p}$ is the product $\omega \cdot w$, with $\omega \in W^{P\left(P^{\prime}\right), p^{\prime}}$ and $w \in W^{R, p}$. We then have canonical isomorphisms

$$
\begin{gathered}
V_{\lambda\left(\ell, w^{\prime}\right)} \stackrel{\sim}{\longrightarrow} V_{\lambda(h, w)(\ell, \omega)} \otimes V_{\lambda(\ell, w)} ; \\
H^{j}\left(X\left(\Gamma_{\ell, R^{\prime}}^{\mathrm{red}}\right), \widetilde{\boldsymbol{V}}_{\lambda\left(\ell, w^{\prime}\right)}\right) \stackrel{\sim}{\longrightarrow} \\
\bigoplus_{i+k=j} H^{k}\left(X\left(\Gamma_{\ell, P\left(P^{\prime}\right)}\right), \tilde{\boldsymbol{V}}_{\lambda(h, w)(\ell, \omega)}\right) \otimes H^{i}\left(X\left(\Gamma_{\ell, R}^{\mathrm{red}}\right), \widetilde{\boldsymbol{V}}_{\lambda(\ell, w)}\right) .
\end{gathered}
$$

Here $V_{\lambda(h, w)(\ell, \omega)}$ is the representation of $G_{\ell, P\left(P^{\prime}\right)}$ associated to the highest weight $\lambda(h, w)$ of $K_{h, P}$ and the Kostant representative $\omega$.

We consider the toroidally compactified (connected) Shimura variety $M_{P, \Sigma(P)}$; let

$$
i_{P\left(P^{\prime}\right)}: Z_{\Sigma(P)}\left(P\left(P^{\prime}\right)\right) \longleftrightarrow M_{P, \Sigma(P)}
$$

denote the inclusion of the closed $P\left(P^{\prime}\right)$ stratum of its boundary. If in (3.3.3) we now let $[\mathcal{B}]$ vary over the $\left[\mathcal{V}_{\lambda(h, w)}\right]$ from $(3.3 .1)$, we conclude that the right-hand side of 
(3.3.2) is the sum over $w$ of the right-hand sides of (3.3.3), tensored with the sum over $i$ of $H^{i}\left(\Gamma_{\ell, R}, V_{\lambda(\ell, w)}\right)$ :

$$
\begin{aligned}
E_{1}^{r+1, s}\left(R^{\prime}\right) \stackrel{\sim}{\longrightarrow} \\
\quad \bigoplus_{w \in W^{R, p}} \bigoplus_{i} E_{1}^{0, s-i-\ell(w)}\left(P\left(P^{\prime}\right) ;\left[\mathcal{V}_{\lambda(h, w)}\right]^{\mathrm{can}}\right) \otimes H^{i}\left(X\left(\Gamma_{\ell, R}^{\mathrm{red}}, \widetilde{\boldsymbol{V}}_{\lambda(\ell, w)}\right)\right) .
\end{aligned}
$$

Here we have written $E_{1}^{0, s}\left(P\left(P^{\prime}\right) ;[\mathcal{B}]\right)$ for the $P\left(P^{\prime}\right)$ piece of the $E_{1}^{0, s}$-term of the nerve spectral sequence calculating the boundary cohomology of $[\mathcal{B}]^{\text {can }}$ on $M_{P, \Sigma(P)}$; it is the cohomology of the pullback of $\left[\mathcal{V}_{\lambda(h, w)}\right]$ to $Z_{\Sigma(P)}\left(P\left(P^{\prime}\right)\right)$.

For use in the next section, we note that the above factorization of $W^{R^{\prime}, p}$ can be continued. As in (3.1), we have Levi subgroups $K_{p}^{P^{\prime},(2)} \subset K_{p}, K_{p^{\prime}}^{P\left(P^{\prime}\right),(2)}$, etc., and the corresponding sets of Kostant representatives. Now we have the factorizations:

$$
\begin{aligned}
W^{R^{\prime}, p} & =W^{P\left(P^{\prime}\right), p^{\prime}} \cdot W^{R, p}=W^{P\left(P^{\prime}\right), p^{\prime}} \cdot W^{P, p} \cdot W_{\ell}^{R}=W^{P \cap P^{\prime}, p} \cdot W_{\ell}^{R} \\
& =W^{Q_{\ell}} \cdot W^{P^{\prime}, p} \cdot W_{\ell}^{R} .
\end{aligned}
$$

Here $W_{\ell}^{R}$ is a set of Kostant representatives for $R \cap G_{\ell, P}$ in $G_{\ell, P}$ and the factorization $W^{R, p}=W^{P, p} \cdot W_{\ell}^{R}$ is the application of Lemma 3.1.6, via Cayley transform, to the Levi subgroup $K_{h, P} \times G_{\ell, R} \subset K_{h, P} \cdot G_{\ell, P}$. Similarly, $W^{P \cap P^{\prime}, p}$ is a set of Kostant representatives in $K_{p}$ obtained by identifying $K_{p}^{P,(2)} \stackrel{\sim}{\longrightarrow} K_{h, P} \times G_{\ell, P}$ and then taking the parabolic subgroup associated to $P\left(P^{\prime}\right)$ in $K_{h, P}$; the last equality on the first line is the application of Lemma 3.1.6 to the pair of inclusions

$$
K_{p^{\prime}}^{P\left(P^{\prime}\right),(2)} \times G_{\ell} \subset K_{h, P} \times G_{\ell} .
$$

Finally, we can identify $K_{p^{\prime}}^{P\left(P^{\prime}\right),(2)} \stackrel{\sim}{\longrightarrow} K_{h, P\left(P^{\prime}\right)} \times G_{\ell, P\left(P^{\prime}\right)}$ via Cayley transform on $G_{h, P}$, and $K_{h, P\left(P^{\prime}\right)}$ is just $K_{h, P^{\prime}}$. Applying Lemma 3.1.6 to the standard parabolic $R_{\ell}^{\prime}$ of $G_{\ell, P^{\prime}}$ with Levi factor $G_{\ell, R^{\prime}}=G_{\ell, R} \times G_{\ell, P\left(P^{\prime}\right)}$, we obtain the factorization $W^{P \cap P^{\prime}, p}=W^{R_{\ell}^{\prime}} \cdot W^{P^{\prime}, p}$, whence the second line.

\subsection{Differentials in the nerve spectral sequence, concluded}

For any automorphic vector bundle $[\mathcal{B}]$ on $M_{P}$, let

$$
d_{P\left(P^{\prime}\right)}: H^{\bullet}\left(M_{P, \Sigma(P)},[\mathcal{B}]^{\text {can }}\right) \longrightarrow H^{\bullet}\left(Z_{\Sigma(P)}\left(P\left(P^{\prime}\right)\right), i_{P\left(P^{\prime}\right)}^{*}[\mathcal{B}]^{\text {can }}\right)
$$

denote the natural restriction. We use the same notation to designate the homomorphism

$$
H^{\bullet}\left(M_{P, \Sigma(P)},[\mathcal{B}]^{\text {can }}\right) \longrightarrow E_{1}^{0, \bullet}\left(P\left(P^{\prime}\right), \mathcal{B}\right)
$$

obtained by composing the restriction map with the decomposition (3.2.3), and analogously in the adelic setting. 
Proposition 3.4.1. - Let $[\mathcal{W}]$ be an automorphic vector bundle on $\operatorname{Sh}(G, X)$, as above, and let $R$ and $R^{\prime}$ be a pair of proper parabolic subgroups of $G$, with $R^{\prime} \subset R$, $r\left(R^{\prime}\right)=r(R)+1$. Suppose that $R$ and $R^{\prime}$ are subordinate to the maximal parabolics $P$ and $P^{\prime}$ respectively $\left(P^{\prime} \prec P\right)$. In terms of the (adelic versions of the) isomorphisms (3.3.1) and (3.3.7), the map $d_{R, R^{\prime}}$ of Corollary (3.2.9) is given by

$$
\bigoplus_{w \in W^{R, p}} \bigoplus_{i} d_{P\left(P^{\prime}\right)} \otimes \mathbf{1}_{i, \lambda(\ell, w)}
$$

where $\mathbf{1}_{i, \lambda(\ell, w)}$ is the identity map of $H^{i}\left(X\left(G_{\ell, R}\right), \tilde{\boldsymbol{V}}_{\lambda(\ell, w)}\right)$.

Proof. - The proposition is stated in the adelic framework, but for the proof it suffices to work with connected Shimura varieties. Let $\bar{Z}_{\sigma} \subset Z_{\Sigma}(R)$ be an irreducible stratum and let $\bar{Z}_{\sigma\left(R^{\prime}\right)}=\bar{Z}_{\sigma} \cap Z_{\Sigma}\left(R^{\prime}\right)=\bar{Z}_{\sigma} \cap Z_{\Sigma}\left(P^{\prime}\right)$. Let $i_{\sigma}$ and $i_{\sigma\left(R^{\prime}\right)}$ be the inclusions of $\bar{Z}_{\sigma}$ and $\bar{Z}_{\sigma\left(R^{\prime}\right)}$, respectively, in $K_{f} \operatorname{Sh}(G, X)_{\Sigma}$.

We will show that the following diagram commutes:

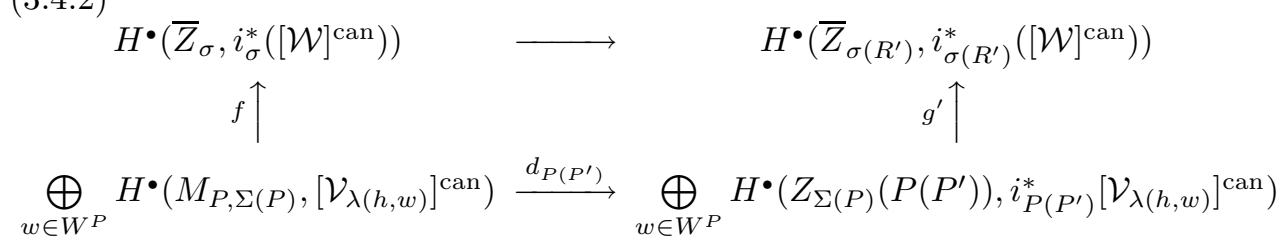

Here the top arrow is the natural restriction map, the left-hand vertical arrow is given by Corollary 1.4.5, and the right-hand vertical arrow is to be constructed. There is difficulty caused by the fact that $\bar{Z}_{\sigma\left(R^{\prime}\right)}$, by which we mean the reduced divisor on $\bar{Z}_{\sigma}$, need not be the pullback of $Z_{\Sigma(P)}\left(P\left(P^{\prime}\right)\right)$.

We abstract the situation and study the restriction map $d_{R^{\prime}, R}$ in the context of base change. Consider the following diagram of morphisms of schemes of finite type over $\mathbb{C}$ :

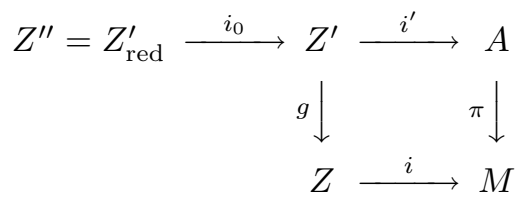

We assume that the square is cartesian. The notation $Z_{\text {red }}^{\prime}$ refers to the underlying reduced scheme of $Z^{\prime}$. The schemes $A$ and $M$ are assumed smooth, and $Z$ is a (reduced) divisor on $M$. The morphism $\pi$ is proper. More generally, we will be later considering situations in which

(3.4.4) (i) $A$ is a finite union of irreducible components, each of which is a smooth scheme;

(ii) any intersection of irreducible components of $A$ is smooth and the restriction of $\pi$ to it is proper. 
In effect, we will take $A$ to be a simplicial scheme, each of whose components is as in (3.4.3).

Let $\mathcal{F}$ be a locally-free sheaf on $A$. Consider the following diagram:

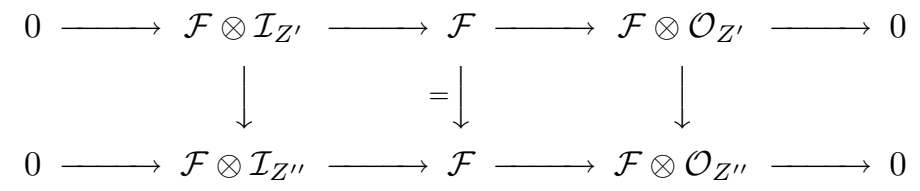

The rows are exact because $\mathcal{F}$ is locally-free. Applying the (exact) functor $R \pi_{*}$ to the first row, we get

$$
0 \longrightarrow R \pi_{*}\left(\mathcal{F} \otimes \mathcal{I}_{Z^{\prime}}\right) \longrightarrow R \pi_{*} \mathcal{F} \longrightarrow R \pi_{*}\left(i^{*} \mathcal{F}\right) \longrightarrow
$$

Since $\mathcal{I}_{Z^{\prime}}=\pi^{*} \mathcal{I}_{Z}$ and $\mathcal{I}_{Z}$ is locally-free, we can rewrite (3.4.6) by "adjunction" as

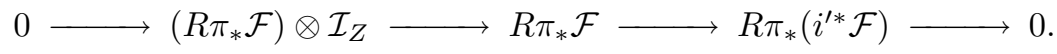

This yields the isomorphism

$$
R \pi_{*}\left(i^{*} \mathcal{F}\right) \approx i^{*} R \pi_{*} \mathcal{F}
$$

We get from (3.4.5):

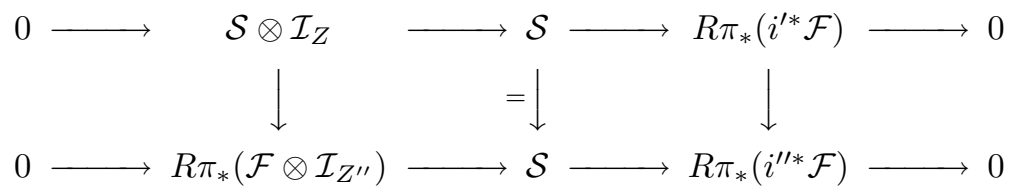

where we have written $\mathcal{S}$ for $R \pi_{*} \mathcal{F}$, and $i^{\prime \prime}=i^{\prime} \circ i_{0}$.

We consider the diagram (3.4.3), where $\pi: A \rightarrow M$ is a morphism of toroidally compactified mixed Shimura varieties $\bullet \operatorname{Sh}(Q, X)_{\Sigma} \longrightarrow . \operatorname{Sh}\left(Q^{\prime}, X^{\prime}\right)_{\Sigma_{1}}$, with $Q^{\prime}=Q / R_{u} Q$ (so $M$ is pure), and $Z$ is the (reduced) boundary divisor associated to a maximal parabolic subgroup of $Q^{\prime}$. Take $\mathcal{F}$ to be a canonically extended automorphic vector bundle $\left[\mathcal{V}^{A}\right]^{\text {can }}$ on $A$ satisfying a Kostant condition, and $\mathcal{S}=R \pi_{*} \mathcal{F}$ again. We want to compare the restriction map $H^{\bullet}(A, \mathcal{F}) \longrightarrow H^{\bullet}\left(Z^{\prime \prime}, i^{\prime \prime}, * \mathcal{F}\right)$ to the map $H^{\bullet}(M, \mathcal{S}) \longrightarrow H^{\bullet}\left(Z, i^{*} \mathcal{S}\right)$.

We are actually interested in the case in (3.4.2): $A=\bar{Z}_{\sigma}, M=M_{P, \Sigma(P)}, Z=$ $Z_{\Sigma(P)}\left(P\left(P^{\prime}\right)\right)$, and $\mathcal{F}=i_{\sigma}^{*}\left([\mathcal{W}]^{\text {can }}\right)$. However, we see by Proposition 1.4.3 that in general we may replace $Q$ by $Q / W_{-2} Q$, thereby reducing the issue to the case where $W_{-2} Q=(0)$. Then $\pi: A \rightarrow M$ above is generically an abelian scheme, and that puts us into the situation of Proposition 2.7.1 (with $P^{\prime}$ replaced here by $P\left(P^{\prime}\right)$ and $\left.Z^{\prime \prime}=Z_{P^{\prime}}^{A}\right)$.

In the present situation, it follows from Proposition 2.7.1 that the left vertical arrow in (3.4.9) is a quasi-isomorphism. This provides the top half of the following 
commutative diagram, where the vertical arrows are just the Leray spectral sequence:

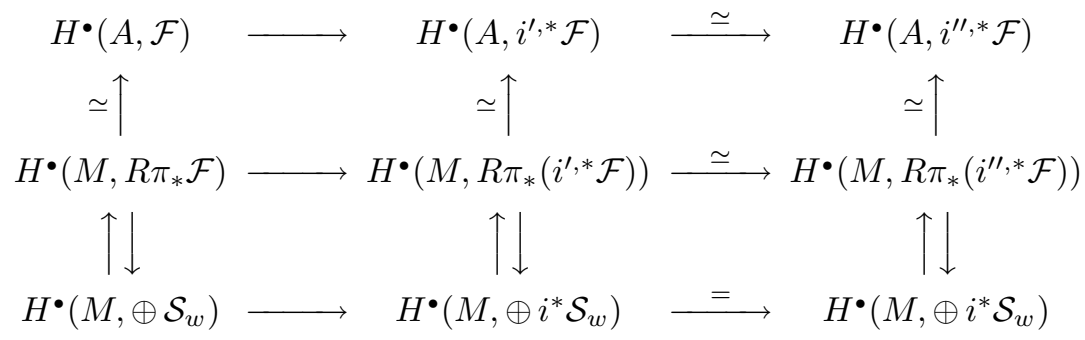

As for the bottom half of (3.4.10), the pairs of opposing vertical maps (all isomorphisms) are given by the splitting in the derived category provided by (2.7.6) and (2.7.7) for all three instances. More precisely, from Lemma (2.7.10) and the subsequent discussion we obtain the left half of the following commutative diagram:

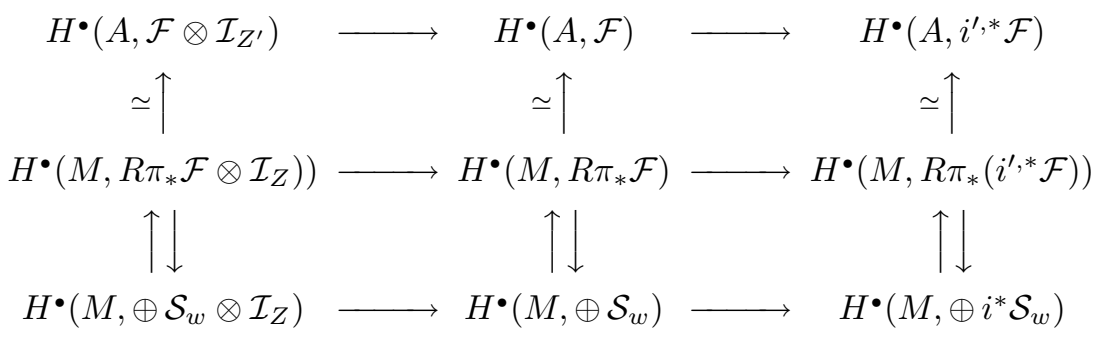

The top of (3.4.11) is obtained from (3.4.7). Using (3.4.8), we can replace $R \pi_{*}\left(i^{\prime, *} \mathcal{F}\right)$ by $i^{*} R \pi_{*} \mathcal{F}$ in the second row of (3.4.11). That the last pair of opposing vertical maps are isomorphisms then follows by the five lemma. It follows that restriction in (3.4.10) respects the splittings. This completes the verification of (3.4.2).

Now the natural projection $Z_{\Sigma(P)}\left(P\left(P^{\prime}\right)\right) \rightarrow M_{P^{\prime}, \Sigma\left(P^{\prime}\right)}$-we are assuming our toroidal compactifications have been chosen compatibly - defines isomorphisms

$$
g^{\prime \prime}: H^{\bullet}\left(Z_{\Sigma(P)}\left(P\left(P^{\prime}\right)\right), i_{P\left(P^{\prime}\right)}^{*}\left[\mathcal{V}_{\lambda(h, w)}\right]^{\text {can }}\right) \stackrel{\sim}{\longrightarrow} E_{1}^{0, \bullet}\left(P\left(P^{\prime}\right),\left[\mathcal{V}_{\lambda(h, w)}\right]\right) .
$$

We claim that the composite

$$
g^{\prime \prime} \circ\left(g^{\prime}\right)^{-1}: H^{\bullet}\left(\bar{Z}_{\sigma\left(R^{\prime}\right)}, i_{\sigma\left(R^{\prime}\right)}^{*}\left([\mathcal{W}]^{\text {can }}\right)\right) \stackrel{\sim}{\longrightarrow} \oplus_{w \in W^{P}} E_{1}^{0, \bullet}\left(P\left(P^{\prime}\right),\left[\mathcal{V}_{\lambda(h, w)}\right]\right)
$$

equals the isomorphism given by applying Proposition 1.4.5 simplicially to the individual closed strata of $\bar{Z}_{\sigma\left(R^{\prime}\right)}$.

More precisely, if $\bar{Z}_{\sigma^{\prime}}$ is an irreducible stratum of $\bar{Z}_{\sigma\left(R^{\prime}\right)}$, then (1.4.5), combined with (3.1.2), asserts an isomorphism between $H^{\bullet}\left(\bar{Z}_{\sigma^{\prime}}, i_{\sigma^{\prime}}^{*}\left([\mathcal{W}]^{\text {can }}\right)\right)$ and a sum over terms indexed by $W^{P^{\prime}, p}$. Similarly, (3.3.3) writes each $E_{1}^{0, \bullet}\left(P\left(P^{\prime}\right),\left[\mathcal{V}_{\lambda(h, w)}\right]\right)$ as a sum of terms indexed by $W^{P\left(P^{\prime}\right), p^{\prime}}$. As in (3.3.8), we have

$$
W^{P\left(P^{\prime}\right), p^{\prime}} \cdot W^{P, p}=W^{P \cap P^{\prime}, p}=W^{R_{\ell}^{\prime}} \cdot W^{P^{\prime}, p} .
$$

Thus this sum can be regrouped as a sum over terms indexed by $W^{P^{\prime}, p}$. The claim, from which the proposition will follow, is that the composite $g^{\prime \prime} \circ\left(g^{\prime}\right)^{-1}$ can be identified with the isomorphism of (1.4.5) after regrouping in this way. But this follows 
formally by applying Proposition 1.5.34 simplicially to the individual closed strata (cf. Remark 1.5.35 (ii)).

We conclude as in [HZ1, §4.8]:

THEOREM 3.4.12. - Let $[\mathcal{W}]$ be an automorphic vector bundle on $\operatorname{Sh}(G, X)$, with canonical model over the field $E(\mathcal{W})$. Suppose $R^{\prime} \subset R$ are two $\mathbb{Q}$-parabolics of $G$ with $r\left(R^{\prime}\right)=r+2=r(R)+1$. Write $E_{1}^{r, s}(R)$ and $E_{1}^{r+1, s}\left(R^{\prime}\right)$ as in Corollary 3.2.9. Then the differential $d_{R, R^{\prime}}: E_{1}^{r, s}(R) \rightarrow E_{1}^{r+1, s}\left(R^{\prime}\right)$ is rational with respect to the $E(\mathcal{W})$-structures on the two sides for all $s$.

Proof. - If $R^{\prime}$ and $R$ are subordinate to two distinct maximal parabolics, this follows from Theorem 4.8.1 of [HZ1] and Proposition 3.4.1, above. If $R^{\prime}$ and $R$ are subordinate to the same maximal parabolic, the theorem is a simple consequence of Corollary 3.2 .9 .

\subsection{Towards the determination of $E_{2}$}

It is a good idea at this point to recall how the nerve spectral sequence (3.2.1) arises as the spectral sequence of a filtered complex. Writing $C^{\bullet}(\cdot)$ for any functorial cochain complex representing $R \Gamma(\cdot)$, one has that $C^{\bullet}\left(i^{*}[\mathcal{W}]^{\text {can }}\right)$ is quasi-isomorphic to the double complex $K^{\bullet}$, with terms

$$
K^{r, s}=\bigoplus_{r(R)=r+1} C^{s}\left(i_{R}^{*}[\mathcal{W}]^{\text {can }}\right),
$$

in which the differential $d$ is the sum of the differential of $C^{\bullet}$ (which increases $s$ by one), and restriction (which increases $r$ by one). We denote the latter by $d_{\text {par }}$. The filtration $\mathcal{R}$ according to the value of $r$ is a decreasing filtration of $K^{\bullet}$, for which

$$
\mathrm{Gr}_{\mathcal{R}}^{t} K^{\bullet}=\underset{r(R)=t+1}{\bigoplus} C^{\bullet}\left(i_{R}^{*}[\mathcal{W}]^{\text {can }}\right)[-t],
$$

in which $d_{\text {par }}$ vanishes. This is the source of the nerve spectral sequence, with $E_{1}$-term $E_{1}\left(K^{\bullet}, \mathcal{R}\right)$, and $d_{1}$ induced by $d_{\text {par }}$.

For comparison later, it is convenient to replace parabolic rank by actual rank. Let $\rho$ denote the rank of $G$ (over $\mathbb{Q}$ ). A $\mathbb{Q}$-parabolic subgroup $R$ of $G$ has $\mathbb{Q}$-rank $\rho_{R}$, with $\rho_{R}+r(R)=\rho$. Thus, $r(R)=t+1$ if and only if $\rho_{R}=\rho-1-t$. The increasing filtration by $\mathbb{Q}$-rank determines the same spectral sequence (up to a translation) that $\mathcal{R}$ does; we allow $\mathcal{R}$ to refer to that from now on.

Our calculations in this Section suggest the following notion. Let $R$ be a parabolic subgroup of $G$ that is subordinate to the maximal parabolic $P$. Then $R$ has a Levi subgroup $G_{h, P} \cdot G_{\ell, R}$.

Definition 3.5.3. - The holomorphic rank of $R$ is the $\mathbb{Q}$-rank of $G_{h, P}$, and is denoted $\rho_{h}(R)$. 
Thus, all parabolic subgroups subordinate to a given $P$ have the same holomorphic rank. We use this notion now to combine (3.2.9) and (3.4.1) into a single statement:

THEOREM 3.5.4. - The differential $d_{\text {par }}$ of $K^{\bullet}$ decomposes as $d_{\text {par }}=d^{\ell}+d^{h}$, where $d^{\ell}$ preserves holomorphic rank and $d^{h}$ lowers holomorphic rank. This induces the same for the differential $d_{1}$ of the $E_{1}$-term $E_{1}\left(K^{\bullet}, \mathcal{R}\right)$ of the nerve (rank) spectral sequence: $d_{1}=d_{1}^{\ell}+d_{1}^{h}$. The determination of $d_{1}^{\ell}$ is given in (3.2.9); that of $d_{1}^{h}$ is given in (3.4.1).

It seems right at this point to shift gears and look instead at the spectral sequence for the filtration $\mathcal{R}^{h}$ of $K^{\bullet}$ by holomorphic rank: $\mathcal{R}_{j}^{h} K^{\bullet}$ is the direct sum of those terms in (3.5.1) with $R$ of holomorphic rank $\leqslant j$. This is clearly closed under $d$. We have

$$
\operatorname{Gr}_{t}^{\mathcal{R}^{h}} K^{\bullet}=\bigoplus_{\rho_{h}(R)=t} C^{\bullet}\left(i_{R}^{*}[\mathcal{W}]^{\mathrm{can}}\right)[1-r(R)]
$$

on which $d^{h}$ vanishes (compare (3.5.2)).

Though we were preoccupied with cohomology calculations in (3.1), we point out that the discussion in (3.1.1) and (3.1.4) actually give assertions in the derived category, viz.,

Proposition 3.5.6. - Let $P$ be a maximal parabolic subgroup of $G$, and $R$ a parabolic subordinate to $P$. Let $\bar{\pi}_{P}: Z_{\Sigma}(R) \rightarrow M_{P, \Sigma(P)}$ be the boundary projection. Then there is a canonical isomorphism in the derived category of $M_{P, \Sigma(P)}$ :

$$
\begin{aligned}
R\left(\bar{\pi}_{P}\right)_{*}\left(i_{R}^{*}[\mathcal{W}]^{\text {can }}\right) & \approx \bigoplus_{w \in W^{P, p}}\left[\mathcal{V}_{\lambda(h, w)}\right]^{\text {can }} \otimes C^{\bullet}\left(\Gamma_{\ell, R}, V_{\lambda(\ell, w)}\right)[-\ell(w)] \\
& \approx \bigoplus_{w \in W^{R, p}}\left[\mathcal{V}_{\lambda(h, w)}\right]^{\text {can }} \otimes C^{\bullet}\left(\Gamma_{\ell, R}^{\mathrm{red}}, V_{\lambda(\ell, w)}\right)[-\ell(w)]^{.}
\end{aligned}
$$

Note that the first factor of the tensor product above is independent of $R$ (for $R$ subordinate to $P$ ). If we combine (3.5.6) with (3.5.5), we obtain something "familiar":

COROLlary 3.5.7. - There is an isomorphism in the derived category of $M_{P, \Sigma(P)}$ :

$$
\operatorname{Gr}_{t}^{\mathcal{R}^{h}} R\left(\bar{\pi}_{P}\right)_{*} K^{\bullet} \approx \bigoplus_{\rho_{h}(P)=t, w \in W^{P, p}}\left[\mathcal{V}_{\lambda(h, w)}\right]^{\operatorname{can}} \otimes K_{c}^{\bullet}\left(X\left(\Gamma_{\ell, P}\right), V_{\lambda(\ell, w)}\right)[-\ell(w)]
$$

where

$$
K_{c}^{\bullet}\left(X\left(\Gamma_{\ell, P}\right), V_{\lambda(\ell, w)}\right)=\bigoplus_{\Pi(R)=P} C^{\bullet}\left(\Gamma_{\ell, R}, V_{\lambda(\ell, w)}\right)
$$

is a cochain complex for the cohomology of the pair $\left(\bar{X}\left(\Gamma_{\ell, P}\right), \partial \bar{X}\left(\Gamma_{\ell, P}\right)\right)$.

As suggested by the notation, one can identify $\left.H^{\bullet}\left(\bar{X}\left(\Gamma_{\ell, P}\right), \partial \bar{X}\left(\Gamma_{\ell, P}\right) ; V_{\lambda(\ell, w)}\right)\right) \simeq$ $\left.H_{c}^{\bullet}\left(X\left(\Gamma_{\ell, P}\right), V_{\lambda(\ell, w)}\right)\right)$. Numbering the standard maximal parabolics by omitted simple root, so that $P_{i} \succ P_{i+1}$, we can now assert: 
COROLlary 3.5.8. - In the spectral sequence for $\mathcal{R}^{h}$, the $E_{1}$-term is

$$
\begin{aligned}
E_{1}^{p, q}\left(K^{\bullet}, \mathcal{R}^{h}\right) & =H^{p+q}\left(\mathrm{Gr}_{-p}^{\mathcal{R}^{h}} K^{\bullet}\right) \\
& =\bigoplus_{a, w ; P \sim P_{\rho+p}} H^{a}\left(M_{P, \Sigma(P)},\left[\mathcal{V}_{\lambda(h, w)}\right]^{\mathrm{can}}\right) \otimes H_{c}^{p+q-a-l(w)}\left(X\left(\Gamma_{\ell, P}\right), \widetilde{V}_{\lambda(\ell, w)}\right) .
\end{aligned}
$$

NB - The non-trivial terms in the above are for $p \leqslant 0$.

Next, we note that the differential $d_{1}$ in (3.5.8) is induced by $d^{h}$. We can deduce the effect of $d_{1}$ by observing that $\mathcal{R}$ is the composite, or convolution, of $\mathcal{R}^{h}$ and an evident analogously defined filtration $\mathcal{R}^{\ell}$, written

$$
\mathcal{R}=\mathcal{R}^{h} * \mathcal{R}^{\ell}
$$

This implies that

$$
\operatorname{Gr}_{j}^{\mathcal{R}} K^{\bullet} \simeq \bigoplus_{t+u=j} \operatorname{Gr}_{t}^{\mathcal{R}^{h}} \operatorname{Gr}_{u}^{\mathcal{R}^{\ell}} K^{\bullet}
$$

(see $[Z 3, \S 1(6)])$. We can see, from the calculations that produced (3.5.4), that $d_{1}$ does "nothing" on the second factor of the tensor product in (3.5.7). In actuality, that means we are looking at the inclusions of $\bar{X}\left(\Gamma_{\ell, P}\right)$ in $\partial \bar{X}\left(\Gamma_{\ell, P^{\prime}}\right)$, with $\rho_{h}\left(P^{\prime}\right)=\rho_{h}(P)-1$. Also, note that there is a cohomology equivalence by excision

$$
\bigsqcup_{P}\left(\bar{X}\left(\Gamma_{\ell, P}\right), \partial \bar{X}\left(\Gamma_{\ell, P}\right)\right) \approx\left(\partial \bar{X}\left(\Gamma_{\ell, P^{\prime}}\right), \partial \bar{X}\left(\Gamma_{\ell, P^{\prime}}\right)-\bigsqcup_{P} X\left(\Gamma_{\ell, P}\right)\right) .
$$

Thus:

Proposition 3.5.11. - In the spectral sequence for $\mathcal{R}^{h}$, the differential

$$
d_{1}: E_{1}^{p, q}\left(K^{\bullet}, \mathcal{R}^{h}\right) \longrightarrow E_{1}^{p+1, q}\left(K^{\bullet}, \mathcal{R}^{h}\right)
$$

is given by

$$
\begin{gathered}
\bigoplus_{a, w ; P}\left\{H^{a}\left(M_{P, \Sigma(P)},\left[\mathcal{V}_{\lambda(h, w)}\right]^{\mathrm{can}}\right) \otimes H_{c}^{p+q-a-l(w)}\left(X\left(\Gamma_{\ell, P}\right), \widetilde{V}_{\lambda(\ell, w)}\right)\right\} \stackrel{d_{1}^{h} \otimes \delta}{\longrightarrow} \\
\bigoplus_{a, w ; P^{\prime}} \underset{\sim P_{\rho+p+1}}{\longrightarrow}\left\{H^{a}\left(M_{P^{\prime}, \Sigma\left(P^{\prime}\right)}, i_{P\left(P^{\prime}\right)}^{*}\left[\mathcal{V}_{\lambda(h, w)}\right]^{\mathrm{can}}\right) \otimes H_{c}^{p+q+1-a-l(w)}\left(X\left(\Gamma_{\ell, P^{\prime}}\right), \widetilde{V}_{\lambda(\ell, w)}\right)\right\} ;
\end{gathered}
$$

in the above, $d_{1}^{h}$ is induced by $d^{h}$ and acts between consecutive boundary components $\left(P \succ P^{\prime}\right)$, and $\delta$ is the connecting homomorphism in the exact sequence of the triple

$$
\left(\bar{X}\left(\Gamma_{\ell, P^{\prime}}\right), \partial \bar{X}\left(\Gamma_{\ell, P^{\prime}}\right), \partial \bar{X}\left(\Gamma_{\ell, P^{\prime}}\right)-\bigsqcup_{P} X\left(\Gamma_{\ell, P}\right)\right)
$$

where $P$ runs over a finite set of conjugates of $P_{\rho+p}$ (see [HZ2, App. to (3.5)]).

The $E_{2}$-term of the holomorphic rank spectral sequence, $E_{2}\left(K^{\bullet}, \mathcal{R}^{h}\right)$, is, of course, isomorphic to the cohomology of $\left(E_{1}\left(K^{\bullet}, \mathcal{R}^{h}\right), d_{1}\right)$. This can be determined as in the appendix below. 
Appendix: Zipper products. - Let $C \cdot$ be a cochain complex with differential $d$, and $V^{\bullet}$ a chain of vector spaces:

$$
V^{0} \stackrel{T_{0}}{\longrightarrow} V^{1} \stackrel{T_{1}}{\longrightarrow} V^{2} \stackrel{T_{2}}{\longrightarrow} \cdots .
$$

For the next definition, one really need assume only that $C \cdot$ is a chain of vector spaces.

Definition 3.A.1. - The zipper product $\operatorname{Zip}\left(C^{\bullet}, V^{\bullet}\right)$ of $C^{\bullet}$ and $V^{\bullet}$ is the chain of vector spaces

$$
V^{0} \otimes C^{0} \stackrel{T_{0} \otimes d}{\longrightarrow} V^{1} \otimes C^{1} \stackrel{T_{1} \otimes d}{\longrightarrow} V^{2} \otimes C^{2} \stackrel{T_{2} \otimes d}{\longrightarrow} \cdots .
$$

The following is easy to verify:

Proposition 3.A.2

(i) If $C^{\bullet}$ is a complex, then $\operatorname{Zip}\left(C^{\bullet}, V^{\bullet}\right)$ is actually a complex.

(ii) $H^{i}\left(\operatorname{Zip}\left(C^{\bullet}, V^{\bullet}\right)\right) \simeq\left\{\left(V^{i} \otimes \operatorname{Ker} d_{i}\right)+\left(\operatorname{Ker} T_{i}\right) \otimes C^{i}\right\} /\left(\operatorname{Im} T_{i-1}\right) \otimes\left(\operatorname{Im} d_{i-1}\right)$.

Our goal is to express the above in terms of the cohomology of $C \bullet$. We point out that (3.5.8) is the direct sum of zipper products. The calculation is based on the unremarkable:

Lemma 3.A.3. - Let $A$ and $B$ be subspaces of a vectorspace, and let $E$ be a subspace of both. Then there is a short exact sequence

$$
0 \longrightarrow A / E \longrightarrow(A+B) / E \longrightarrow(A+B) / A \simeq B /(A \cap B) \longrightarrow 0 .
$$

In our case (3.A.2)(ii), we compute

$$
A / E=\left(V^{i} \otimes \operatorname{Ker} d_{i}\right) /\left(\operatorname{Im} T_{i-1}\right) \otimes\left(\operatorname{Im} d_{i-1}\right) \simeq\left(\operatorname{Im} T_{i-1}\right) \otimes H^{i}\left(C^{\bullet}\right) \oplus\left(\tilde{V}^{i} \otimes \operatorname{Ker} d_{i}\right),
$$

where $\widetilde{V}^{i}$ is a complement to $\operatorname{Im} T_{i-1}$ in $V^{i}$. On the other hand,

$B /(A \cap B)=\left\{\left(\operatorname{Ker} T_{i}\right) \otimes C^{i}\right\} /\left\{\left[\left(\operatorname{Ker} T_{i}\right) \otimes C^{i}\right] \cap\left[V^{i} \otimes\left(\operatorname{Ker} d_{i}\right)\right]\right\}=\left(\operatorname{Ker} T_{i}\right) \otimes\left(C^{i} / \operatorname{Ker} d_{i}\right)$.

Putting these together, we obtain:

Proposition 3.A.4

$$
\begin{aligned}
& H^{i}\left(\operatorname{Zip}\left(C^{\bullet}, V^{\bullet}\right)\right) \\
& \simeq\left\{\left(\operatorname{Im} T_{i-1}\right) \otimes H^{i}\left(C^{\bullet}\right)\right\} \oplus\left\{\left(\operatorname{Coker} T_{i-1}\right) \otimes\left(\operatorname{Ker} d_{i}\right)\right\} \oplus\left\{\left(\operatorname{Ker} T_{i}\right) \otimes\left(C^{i} / \operatorname{Ker} d_{i}\right)\right\} .
\end{aligned}
$$

\subsection{Differentials and automorphic forms}

It remains to express the $E_{1}$ terms and the differentials $d_{1}$ in terms of automorphic forms. The terms $E_{1}^{1, s}$ have already been treated in [HZ1, $\left.\$ 4.2\right]$. The general case is completely analogous, and we will simply state the result. However, Franke's theorem [Fr1] justifies replacing slowly increasing smooth functions by automorphic forms in the Lie algebra cohomology groups, and we will do so. We warn the reader that 
[Fr1] only treats (topological) cohomology with local coefficients. The extension to coherent cohomology is contained in an unpublished early version of [Fr1].

Suppose $H$ is a reductive algebraic group over $\mathbb{Q}, P \subset H$ a parabolic subgroup, $N \subset P$ its unipotent radical, $L=P / N$ the Levi quotient. The constant term map $c_{H, P}: \mathcal{A}(H) \rightarrow \mathcal{A}(L)$ is defined as usual:

$$
c_{H, P}(f)(g)=\int_{N(\mathbb{Q}) \backslash N(\boldsymbol{A})} f(n g) d n,
$$

where $d n$ is the right-invariant measure on $N(\mathbb{Q}) \backslash N(\boldsymbol{A})$ with total mass 1 . If now $R^{\prime} \subset R$ is a pair of parabolic subgroups of $G$, with Levi quotients $L_{R^{\prime}}$ and $L_{R}$, respectively, we write $c_{R, R^{\prime}}$ for $c_{L_{R}, L_{R^{\prime}}}$.

As in [HZ1, $\S 4$, we write $I_{R}^{G}=\operatorname{Ind}_{R\left(\boldsymbol{A}_{f}\right)}^{G\left(\boldsymbol{A}_{f}\right)}$, when $R$ is a parabolic subgroup of $G$. Given $R$ subordinate to the maximal parabolic $P$, we write $\mathfrak{p}_{h, R}^{-}=\mathfrak{p}_{h, P}^{-}$. Let

$$
\mathfrak{g}_{\ell, R}=\mathfrak{k}_{\ell, R} \oplus \widetilde{\mathfrak{p}}_{\ell, R}
$$

be the (complex) Cartan decomposition. As in [HZ1, 4.2$]$, we write

$$
\widetilde{\mathfrak{p}}_{\ell, R}=\mathfrak{a}_{R} \oplus \mathfrak{p}_{\ell, R},
$$

where $\mathfrak{a}_{R}$ is the Lie algebra of a split component $A_{R}$ of $G_{\ell}$ containing the center $Z_{G}$ of $G$. Finally, write $\mathfrak{s}_{R}$ instead of $\mathfrak{s}_{p, R}$, and let $\mathfrak{u}_{P}(R)$ denote the Lie algebra of the unipotent radical of $R_{\ell, P}$. Then we can decompose

$$
\mathfrak{s}_{R} \stackrel{\sim}{\longrightarrow} \mathfrak{s}_{P} \oplus \mathfrak{u}_{P}(R)
$$

Recall from $[\mathrm{HZ1},(3.10 .3 .2),(4.2 .6)]$ that we can use the Cayley transform to identify

$$
\mathfrak{p}^{-} \stackrel{\sim}{\longrightarrow} \mathfrak{p}_{h, P}^{-} \oplus \mathfrak{u}_{P, \mathbb{C}} \oplus \mathfrak{v}_{P}^{-}
$$

It is better for our purposes to use the further identification

$$
\mathfrak{p}^{-} \stackrel{\sim}{\longrightarrow} \mathfrak{p}_{h, P}^{-} \oplus \tilde{\mathfrak{p}}_{\ell, P} \oplus \mathfrak{s}_{P}
$$

derived from $[\mathrm{HZ1},(1.8 .6)]$. Now projection of $\operatorname{Lie}\left(R_{\ell, P}\right)=\mathfrak{g}_{\ell, R} \oplus \mathfrak{u}_{P}(R)$ onto $\widetilde{\mathfrak{p}}_{\ell, P}$ via the Cartan decomposition for $\mathfrak{g}_{\ell, P}$ defines an isomorphism of $K_{\ell, R^{-}}$modules

$$
\tilde{\mathfrak{p}}_{\ell, R} \oplus \mathfrak{u}_{P}(R) \stackrel{\sim}{\longrightarrow} \widetilde{\mathfrak{p}}_{\ell, P} .
$$

Combining (3.6.3-3.6.5), we obtain an isomorphism of $K_{h, R} \cdot K_{\ell, R} \cdot A_{R}(\mathbb{R})$-modules

$$
\mathfrak{p}^{-} \stackrel{\sim}{\longrightarrow} \mathfrak{p}_{h, P}^{-} \oplus \widetilde{\mathfrak{p}}_{\ell, R} \oplus \mathfrak{s}_{R} .
$$

Now 
74 CHAPTER 3. THE NERVE SPECTRAL SEQUENCE FOR COHERENT COHOMOLOGY

THEOREM 3.6.7

(i) For each $r, s$, there is an isomorphism

$$
\begin{aligned}
E_{1}^{r, s} \stackrel{\sim}{\longrightarrow} & \left.\underset{r(R)=r+1}{\bigoplus} I_{R}^{G}\left\{\bigoplus_{w \in W^{R, p}}\left[\wedge \cdot\left(\mathfrak{p}_{h, R}^{-} \oplus \widetilde{\mathfrak{p}}_{\ell, R}\right)^{*} \otimes \mathcal{A}\left(L_{R}\right) \otimes H^{\bullet}\left(\mathfrak{s}_{R}, V_{\lambda}\right)\right]^{K_{h} \cdot K_{\ell, R} \cdot A_{R}(\mathbb{R})}\right]\right\}^{s}
\end{aligned}
$$

(on the right-hand side: terms of total degree $s$ ).

(ii) For each $r, s$, there is a commutative diagram

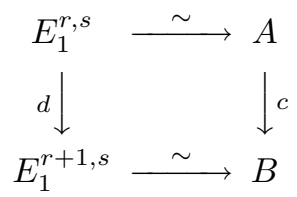

with $A \stackrel{c}{\longrightarrow} B$ given by

$$
\begin{gathered}
\bigoplus_{r(R)=r+1} I_{R}^{G}\left\{\bigoplus_{w \in W^{R, p}}\left[\wedge^{\bullet}\left(\mathfrak{p}_{h, R}^{-} \oplus \widetilde{\mathfrak{p}}_{\ell, R}\right)^{*} \otimes \mathcal{A}\left(L_{R}\right) \otimes H^{\bullet}\left(\mathfrak{s}_{R}, V_{\lambda}\right)\right]^{\left.K_{h} \cdot K_{\ell, R} \cdot A_{R}(\mathbb{R})\right]}\right\}^{s} \\
\bigoplus_{R^{\prime} \subsetneq R, r\left(R^{\prime}\right)=r(R)+1=r+2} c_{R, R^{\prime}} \\
\bigoplus_{r\left(R^{\prime}\right)=r+2} I_{R^{\prime}}^{G}\left\{\bigoplus_{w \in W^{R^{\prime}, p}}\left[\wedge \cdot\left(\mathfrak{p}_{h, R^{\prime}}^{-} \oplus \widetilde{\mathfrak{p}}_{\ell, R^{\prime}}\right)^{*} \otimes \mathcal{A}\left(L_{R^{\prime}}\right) \otimes H^{\bullet}\left(\mathfrak{s}_{R^{\prime}}, V_{\lambda}\right)\right]^{\left.K_{h} \cdot K_{\ell, R^{\prime}} \cdot A_{R^{\prime}}(\mathbb{R})\right]}\right\}^{s} .
\end{gathered}
$$

Here the constant term maps are applied to functions, and the map on coefficients:

$$
\left[\wedge^{\bullet}\left(\mathfrak{p}_{h, R}^{-} \oplus \widetilde{\mathfrak{p}}_{\ell, R}\right)^{*} \otimes H^{\bullet}\left(\mathfrak{s}_{R}, V_{\lambda}\right)\right] \longrightarrow\left[\wedge^{\bullet}\left(\mathfrak{p}_{h, R^{\prime}}^{-} \oplus \widetilde{\mathfrak{p}}_{\ell, R^{\prime}}\right)^{*} \otimes H^{\bullet}\left(\mathfrak{s}_{R^{\prime}}, V_{\lambda}\right)\right]
$$

is obtained by applying (3.6.6) to identify

$$
\wedge^{\bullet}\left(\mathfrak{p}_{h, R}^{-} \oplus \widetilde{\mathfrak{p}}_{\ell, R} \oplus \mathfrak{s}_{R}\right)^{*} \stackrel{\sim}{\longrightarrow} \wedge \cdot\left(\mathfrak{p}^{-}\right)^{*},
$$

and likewise for $R^{\prime}$.

Proof. - That this holds for the $d_{R, R^{\prime}}$ with $R$ and $R^{\prime}$ subordinate to the same maximal parabolic follows from Corollary 3.2.9 and [Sch,1.10]. For $R$ and $R^{\prime}$ subordinate to distinct maximal parabolics, this follows from Proposition 3.4.1 and diagram (4.2.4) of [HZ1]. 


\section{CHAPTER 4}

\section{HODGE THEORETIC APPLICATIONS}

In this Section, we will tie together the results and methods of [HZ1] (as generalized here in $\S 3$ ) with the Hodge theoretic considerations from [HZ2].

\subsection{The Hodge filtration for boundary cohomology}

Let $(G, X)$ be a pair defining a (pure) Shimura variety, $E$ a finite-dimensional vector space over $\mathbb{Q}$, and $\rho: G \rightarrow G L(E)$ a homomorphism of algebraic groups. This determines a compatible family of local systems $\widetilde{\boldsymbol{E}}$ on the associated complex varieties $\operatorname{Sh}(G, X)(\mathbb{C})$. Moreover, the space $X$, by its nature (see (1.1)), defines a family of variations of Hodge structure on $\operatorname{Sh}(G, X)(\mathbb{C})$, with underlying local system $\widetilde{\boldsymbol{E}}$.

Let $M=M_{\Gamma}$ denote a connected component of $\operatorname{Sh}(G, X)(\mathbb{C})$. Let $j: M \rightarrow M_{\Sigma}$ be the inclusion of $M$ in a smooth toroidal compactification, and let $Z_{\Sigma}$ denote the corresponding boundary divisor $\partial M_{\Sigma}$, a divisor with normal crossings in $M_{\Sigma}$. This implies that the holomorphic logarithmic complex,

$$
\Omega_{M_{\Sigma}}^{\bullet}\left(\log Z_{\Sigma}\right) \otimes \widetilde{\mathcal{E}}^{\text {can }}
$$

is quasi-isomorphic to $R j_{*} \widetilde{\boldsymbol{E}}$. In the above, $\widetilde{\mathcal{E}}^{\text {can }}$ denotes the canonical extension of the flat vector bundle $\mathcal{E}$ determined by $E$, as in [HZ1, 4.4]. The complex (4.1.1) is equipped with the decreasing Hodge filtration $F$, and the increasing weight filtration $W$ induced from one on $R j_{*} \widetilde{\boldsymbol{E}}$. These complete the data of a cohomological mixed Hodge complex that induces, upon taking hypercohomology, the mixed Hodge structure on $H^{\bullet}(M, \widetilde{\boldsymbol{E}})^{(1)}$.

Though the description of $W$ on (4.1.1) is rather complicated, it is easy to specify $F$, and we will study the latter. Let $\left\{F^{p} \Omega_{M}^{\bullet}\left(\log Z_{\Sigma}\right)\right\}$ be the usual Hodge filtration, given simply by truncation from below, and let $\left\{\mathcal{F}^{s}\right\}$ be the filtration of $\mathcal{E}$ that gives

\footnotetext{
(1) It may be useful to keep in mind that this remains true for admissible variations of mixed Hodge structure [Sa2].
} 
the variation of Hodge structure. Then the filtration $F$ of (4.1.1) is given by the tensor product of these two:

$$
F^{p}\left(\Omega_{M_{\Sigma}}\left(\log Z_{\Sigma}\right) \otimes \widetilde{\mathcal{E}}^{\mathrm{can}}\right)=\sum_{r+s=p} F^{r} \Omega_{M_{\Sigma}}^{\bullet}\left(\log Z_{\Sigma}\right) \otimes\left(\mathcal{F}^{s}\right)^{\text {can }} .
$$

For this, there is a general definition of $\left(\mathcal{F}^{s}\right)^{\text {can }}$ for a variation of Hodge structure, given by $j_{*} \mathcal{F}^{s} \cap \widetilde{\mathcal{E}}^{\text {can }}$. In the present case, each $\mathcal{F}^{s}$ is an automorphic vector bundle and $\left(\mathcal{F}^{s}\right)^{\text {can }}$ coincides with the canonical extension in the sense of automorphic vector bundles (see [HZ1,3.2]). Because $\left(\Omega_{M}^{p}\right)^{\text {can }}$ is $\Omega_{M_{\Sigma}}^{p}\left(\log Z_{\Sigma}\right)$, we can rewrite (4.1.2) as:

$$
F^{p}\left(\Omega_{M_{\Sigma}}\left(\log Z_{\Sigma}\right) \otimes \widetilde{\mathcal{E}}^{\mathrm{can}}\right)=\sum_{r+s=p}\left(F^{r} \Omega_{M}^{\bullet} \otimes \mathcal{F}^{s}\right)^{\text {can }} .
$$

By Hodge theory [De2, (8.1.9)], the spectral sequence for $F$ degenerates at $E_{1}$.

There are parallel assertions for deleted neighborhood cohomology. For each admissible parabolic subgroup $R$ of $G$, let $i_{R}: Z_{\Sigma}(R) \rightarrow M_{\Sigma}$ denote the inclusion of the $R$-stratum of $\partial M_{\Sigma}$. The complex

$$
\mathcal{C}_{\mathrm{dn}}\left(Z_{\Sigma}(R), \widetilde{\boldsymbol{E}}\right)=i_{R}^{*} R j_{*} \widetilde{\boldsymbol{E}}
$$

is called the deleted neighborhood complex for $Z_{\Sigma}(R)$ in $M_{\Sigma}$, for its hypercohomology is naturally isomorphic to

$$
H_{\mathrm{dn}}\left(Z_{\Sigma}(R), \widetilde{\boldsymbol{E}}\right)=H^{\bullet}\left(N(R)-Z_{\Sigma}(R), \widetilde{\boldsymbol{E}}\right),
$$

where $N(R)$ is a regular neighborhood of $Z_{\Sigma}(R)$ in $M_{\Sigma}$. For $R^{\prime}$ parabolic in $R$, let

$$
i_{R^{\prime}, R}: Z_{\Sigma}\left(R^{\prime}\right) \longrightarrow Z_{\Sigma}(R)
$$

denote the inclusion; note that the evident relation $i_{R^{\prime}}=i_{R^{\prime}, R} \circ i_{R}$ yields:

$$
\mathcal{C}_{\mathrm{dn}}\left(Z_{\Sigma}\left(R^{\prime}\right), \widetilde{\boldsymbol{E}}\right) \approx i_{R^{\prime}, R}^{*} \mathcal{C}_{\mathrm{dn}}\left(Z_{\Sigma}(R), \widetilde{\boldsymbol{E}}\right) .
$$

It is not hard to see that the following complex is quasi-isomorphic to $\mathcal{C}_{\mathrm{dn}}\left(Z_{\Sigma}(R), \widetilde{\boldsymbol{E}}\right)$ :

$$
i_{R}^{*}\left(\Omega_{M_{\Sigma}}(\log Z) \otimes \widetilde{\mathcal{E}}^{\mathrm{can}}\right),
$$

the complex of locally-free sheaves on $Z_{\Sigma}(R)$ given by restricting (4.1.1). This complex inherits a filtration $F$ from (4.1.2). Again, for general reasons, the filtration $F$ induces the Hodge filtration of the natural mixed Hodge structure on $H_{\mathrm{dn}}\left(Z_{\Sigma}(R), \widetilde{\boldsymbol{E}}\right)$ [Sa3]; and the spectral sequence for $F$ degenerates at $E_{1}$.

We now introduce the notation

$$
\mathcal{D R}(M, \widetilde{\boldsymbol{E}})=\Omega_{M}^{\bullet} \otimes \widetilde{\boldsymbol{E}} \simeq \mathcal{C} \cdot\left(\mathfrak{p}^{+}, E\right),
$$

so that, for instance, (4.1.1) becomes $\mathcal{D} \mathcal{R}(M, \widetilde{\boldsymbol{E}})^{\text {can }}$ (cf. (4.1.3)). This is a complex of automorphic vector bundles that are fully decomposed only when the representation $\rho$ is trivial. We need to determine the direct images of deleted neighborhood complexes, first in the unfiltered category (the filtered version will be deduced later, in (4.3)): 
Proposition 4.1.9. - Let $P$ be a maximal $\mathbb{Q}$-parabolic subgroup of $G$, and $R$ a parabolic subordinate to $P$. Let $\bar{\pi}_{P}: Z_{\Sigma}(R) \rightarrow\left(M_{P}\right)_{\Xi}$ be the boundary projection. Then there is a canonical isomorphism in the derived category of $\left(M_{P}\right)_{\Xi}$ :

$$
R\left(\bar{\pi}_{P}\right)_{*}\left(i_{R}^{*}\right)\{\mathcal{D} \mathcal{R}(M, \widetilde{\boldsymbol{E}})\}^{\mathrm{can}} \approx\left\{\mathcal{D} \mathcal{R}\left(M_{P}, \widetilde{\boldsymbol{C}}^{\bullet}\left(\Gamma_{\ell, R}, H^{\bullet}\left(\mathfrak{w}_{P}, E\right)\right)\right)\right\}^{\mathrm{can}} .
$$

Proof. - Using the composite $\bar{\pi}_{P}=\bar{\pi}_{1} \circ \bar{\pi}_{2}$, as per (1.4.2), we calculate that:

$$
\begin{aligned}
R\left(\bar{\pi}_{P}\right)_{*}\left(i_{R}^{*}\{\mathcal{D} \mathcal{R}\right. & \left.(M, \widetilde{\boldsymbol{E}})\}^{\text {can }}\right) \approx R \bar{\pi}_{1, *}\left(\bar{\pi}_{2, *} i_{R}^{*} \mathcal{C}^{\bullet}\left(\mathfrak{p}^{+}, E\right)^{\text {can }}\right) \quad(\text { by }(4.1 .8)) \\
& \approx \mathcal{C} \bullet\left(\Gamma_{\ell, R}, C^{\bullet}\left(\mathfrak{s}_{P}^{\prime},\left(C^{\bullet}\left(\mathfrak{p}^{+}, E\right)\right)\right)\right)^{\text {can }} \quad(\text { by }(2.7 .5) \text { and }(3.5 .6)) \\
& \approx \mathcal{C} \bullet\left(\Gamma_{\ell, R}, C^{\bullet}\left(\mathfrak{s}_{P}^{\prime} \oplus \mathfrak{p}^{+}, E\right)\right)^{\text {can }} \quad(\text { by Remark } 3.1 .9)
\end{aligned}
$$

where $\mathfrak{s}_{P}^{\prime}$ denotes the complex conjugate of $\mathfrak{s}_{P}$. The Cayley transform switches us from $G$ to $G_{h, P}$ as follows:

$$
\begin{aligned}
& \left.R\left(\bar{\pi}_{P}\right)_{*}\left(i_{R}^{*}\right)\{\mathcal{D} \mathcal{R}(M, \widetilde{\boldsymbol{E}})\}^{\text {can }}\right) \approx \mathcal{C}^{\bullet}\left(\Gamma_{\ell, R}, C^{\bullet}\left(\mathfrak{s}_{P}^{\prime} \oplus \mathfrak{p}^{+}, E\right)\right)^{\text {can }} \quad \text { (repeated from above) } \\
& \left.\approx \mathcal{C}^{\bullet}\left(\Gamma_{\ell, R}, C^{\bullet}\left(\mathfrak{p}_{h, P}^{+} \oplus \mathfrak{w}_{P}, E\right)\right)^{\text {can }} \quad \text { (induced by } \bar{c}_{P}\right) \\
& \approx \mathcal{C}^{\bullet}\left(\Gamma_{\ell, R}, C^{\bullet}\left(\mathfrak{p}_{h, P}^{+}, C^{\bullet}\left(\mathfrak{w}_{P}, E\right)\right)\right)^{\text {can }} \quad((3.1 .9) \text { again }) \\
& \approx \mathcal{C}^{\bullet}\left(\mathfrak{p}_{h, P}^{+}, C^{\bullet}\left(\Gamma_{\ell, R}, C^{\bullet}\left(\mathfrak{w}_{P}, E\right)\right)\right)^{\text {can }} \quad\left(\text { as } G_{h, P} \text { and } G_{\ell, R} \text { commute }\right) \\
& \approx\left\{\mathcal{D} \mathcal{R}\left(M_{P}, \widetilde{\boldsymbol{C}}^{\bullet}\left(\Gamma_{\ell, R}, C^{\bullet}\left(\mathfrak{w}_{P}, E\right)\right)\right)\right\}^{\text {can }} \quad \text { (equivalent formula) } \\
& \approx\left\{\mathcal{D R}\left(M_{P}, \widetilde{\boldsymbol{C}}^{\bullet}\left(\Gamma_{\ell, R}, H^{\bullet}\left(\mathfrak{w}_{P}, E\right)\right)\right)\right\}^{\text {can }} \quad \text { (follows by Kostant; cf. (2.7.5)). }
\end{aligned}
$$

\section{REMARKS 4.1.10}

(i) Recall (cf. $[\mathrm{HZ1}, 1.8 .3])$ that conjugation by $c_{P}^{-1}$ takes a subgroup $K_{t}$ of $K_{p}$ onto $G_{\ell}$, and transforms the adjoint action of $K_{t}$ on $\mathfrak{s}_{P} \oplus \mathfrak{p}^{-}$to the action of $G_{\ell}$ on $\mathfrak{p}_{h, P}^{-} \oplus \mathfrak{w}_{P}$. Thus the action of $\Gamma_{\ell, R}$ on $C \cdot\left(\mathfrak{s}_{P} \oplus \mathfrak{p}^{+}, E\right)$ in the first line above comes from the identification of $\Gamma_{\ell, R}$, via $\bar{c}_{P}=c_{P}^{-1}$, with a subgroup of $K_{p}$, or equivalently, the identification

$$
C^{\bullet}\left(\mathfrak{s}_{P}^{\prime} \oplus \mathfrak{p}^{+}, E\right) \simeq C^{\bullet}\left(\mathfrak{p}_{h, P}^{+} \oplus \mathfrak{w}_{P}, E\right)
$$

The above is the basis of the calculations in [HZ1] that underlie this isomorphism (see [HZ1, Lemma 3.5.11]). The action on the second line of the above series of quasiisomorphisms, by contrast, is via the usual (adjoint) action of $G_{\ell, R}$ on $\mathfrak{w}_{P} ; G_{\ell, R}$ and $\bar{c}_{P}$ both act as the identity on $\mathfrak{p}_{h, P}^{+}$.

Moreover, for $P^{\prime} \prec P$, one has $c_{P^{\prime}}=c_{P} \circ c_{P^{\prime}, P}$, where $c_{P^{\prime}, P}$ denotes the Cayley transform for $\widehat{x}_{P^{\prime}}$ as a boundary component of $\widehat{x}_{P}$.

(ii) Note that we have elected not to make use of the Kostant decomposition of $H \bullet\left(\mathfrak{s}_{P}, V\right)$ (thus also its complex conjugate), utilized in [HZI, 3.6] and Section 3 here. The conclusion of the above result can be viewed as the extension of (3.5.6) to special complexes that are not fully decomposed. Also, the final answer is, as one expects 
after [HZ1], independent of the choice of toroidal compactification. Introducing the Kostant decomposition,

$$
H^{\bullet}\left(\mathfrak{w}_{P}, E\right)=\bigoplus_{w \in W^{P}} E_{\mu(h, w)} \otimes E_{\mu(\ell, w)}[-l(w)]
$$

we can rewrite the formula of (4.1.9) as:

$$
\begin{aligned}
R\left(\bar{\pi}_{P}\right)_{*} i_{R}^{*} R j_{*} \widetilde{\boldsymbol{E}} & \approx \bigoplus_{w \in W^{P}} R\left(j_{P}\right)_{*} \widetilde{\boldsymbol{E}}_{\mu(h, w)} \otimes H^{\bullet}\left(\Gamma_{\ell, R}, E_{\mu(\ell, w)}\right)[-l(w)] \\
& \approx\left\{\mathcal{D} \mathcal{R}\left(M_{P}, \widetilde{\boldsymbol{L}}_{R}\right)\right\}^{\mathrm{can}}
\end{aligned}
$$

where $j_{P}: M_{P} \hookrightarrow\left(M_{P}\right)_{\Xi}$ is the inclusion, and $\left.L_{R}=H^{\bullet}\left(\Gamma_{\ell, R}, H \bullet\left(\mathfrak{w}_{P}, E\right)\right)\right)$.

(iii) Throughout, we can express the $\Gamma_{\ell, R^{-c o h o m o l o g y}}$ as $\Gamma_{\ell, R}^{\text {red }}$-cohomology, using the maneuver that produced (3.1.8). We would then write

$$
L_{R}=H^{\bullet}\left(\Gamma_{\ell, R}^{\mathrm{red}}, H^{\bullet}\left(\mathfrak{w}_{R}, E\right)\right)
$$

Next, let $R^{\prime}=R \cap P^{\prime}$, with $P^{\prime}$ maximal. We consider the restriction mappings from $Z_{R}$ to $Z_{R^{\prime}}$ in conjunction with Proposition (4.1.9). If $P^{\prime} \succ P$, then $R^{\prime}$ is also subordinate to $P$. In that case,

$$
R\left(\bar{\pi}_{P}\right)_{*}\left(\left(i_{R}^{*}\right)\{\mathcal{D} \mathcal{R}(M, \widetilde{\boldsymbol{E}})\}^{\mathrm{can}}\right) \longrightarrow R\left(\bar{\pi}_{P}\right)_{*}\left(i_{R^{\prime}}^{*}\{\mathcal{D} \mathcal{R}(M, \widetilde{\boldsymbol{E}})\}^{\mathrm{can}}\right)
$$

is given tautologically:

$$
\begin{aligned}
& \left\{\mathcal{D} \mathcal{R}\left(M_{P}, \widetilde{\boldsymbol{C}}^{\bullet}\left(\Gamma_{\ell, R}, H^{\bullet}\left(\mathfrak{w}_{P}, E\right)\right)\right)\right\}^{\mathrm{can}} \longrightarrow \\
& \left\{\mathcal{D} \mathcal{R}\left(M_{P}, \widetilde{\boldsymbol{C}}^{\bullet}\left(\Gamma_{\ell, R^{\prime}}, H^{\bullet}\left(\mathfrak{w}_{P}, E\right)\right)\right)\right\}^{\text {can }},
\end{aligned}
$$

which is induced by the morphism of local systems coming by restriction on the $\Gamma_{\ell}$ 's. After grading for $F$, the above is essentially equivalent to what we discussed in (3.2). On the other hand, if $P^{\prime} \prec P$, then $R^{\prime}$ is subordinate to $P^{\prime}$. In that case, one reverts to the determination in (3.4).

The treatment of $d^{h}$ has the same problem and the same remedy as before. Consider the diagram:

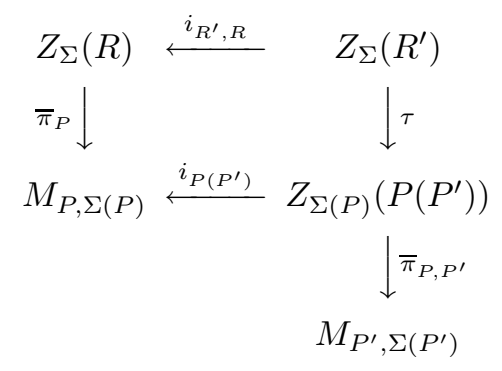


Let $L_{R}^{\bullet}=C^{\bullet}\left(\Gamma_{\ell, R}, H \bullet\left(\mathfrak{w}_{P}, E\right)\right)$. Then the calculation:

$$
\begin{aligned}
R\left(\bar{\pi}_{P^{\prime}}\right)_{*}\left(i_{R^{\prime}}^{*}\{\right. & \left.\mathcal{D} \mathcal{R}(M, \widetilde{\boldsymbol{E}})\}^{\mathrm{can}}\right)=R\left(\bar{\pi}_{P, P^{\prime}}\right)_{*} R \tau_{*}\left(i_{R^{\prime}, R}^{*} i_{R}^{*}\right)\{\mathcal{D} \mathcal{R}(M, \widetilde{\boldsymbol{E}})\}^{\text {can }} \\
& \approx R\left(\bar{\pi}_{P, P^{\prime}}\right)_{*} i_{P\left(P^{\prime}\right)}^{*} R\left(\bar{\pi}_{P}\right)_{*} i_{R}^{*}\{\mathcal{D} \mathcal{R}(M, \widetilde{\boldsymbol{E}})\}^{\mathrm{can}} \quad(\text { from }(2.7 .11)) \\
& \approx R\left(\bar{\pi}_{P, P^{\prime}}\right)_{*} i_{P\left(P^{\prime}\right)}^{*}\left\{\mathcal{D} \mathcal{R}\left(M_{P}, \widetilde{\boldsymbol{L}}_{R}\right)\right\}^{\mathrm{can}} \quad(\text { by }(4.1 .9)) \\
& \approx\left\{\mathcal{D} \mathcal{R}\left(M_{P^{\prime}}, \widetilde{\boldsymbol{C}}^{\bullet}\left(\Gamma_{\ell, P\left(P^{\prime}\right)}, H^{\bullet}\left(\mathfrak{u}_{P\left(P^{\prime}\right)}, L_{R}\right)\right)\right)\right\}^{\text {can }} \\
& =\left\{\mathcal { D } \mathcal { R } \left(M_{P^{\prime}}, \widetilde{\boldsymbol{C}}^{\bullet}\left(\Gamma_{\ell, P\left(P^{\prime}\right)}, H^{\bullet}\left(\mathfrak{u}_{P\left(P^{\prime}\right)}, H^{\bullet}\left(\Gamma_{\ell, R}, H^{\bullet}\left(\mathfrak{w}_{P}, E\right)\right)\right)\right\}^{\mathrm{can}}\right.\right. \\
& \simeq\left\{\mathcal{D} \mathcal{R}\left(M_{P^{\prime}}, \widetilde{\boldsymbol{C}}^{\bullet}\left(\Gamma_{\ell, R^{\prime}}, H^{\bullet}\left(\mathfrak{w}_{P^{\prime}}, E\right)\right)\right)\right\}^{\mathrm{can} \quad(\text { as in }(3.1 .6))}
\end{aligned}
$$

recovers the direct computation of $R\left(\bar{\pi}_{P^{\prime}}\right)_{*} i_{R^{\prime}}^{*}\{\mathcal{D} \mathcal{R}(M, \widetilde{\boldsymbol{E}})\}^{\text {can }}$, because of $(4.1 .10)$ and the way in which the Cayley transforms entered. We now see that $d^{h}$ is induced by restriction on the $M_{P}$ 's.

When one grades (4.1.2) for $F$, the differentials become linearized, and each $\operatorname{Gr}_{F}^{p}\left(\Omega_{M_{\Sigma}}\left(\log Z_{\Sigma}\right) \otimes \widetilde{\mathcal{E}}^{\text {can }}\right)$ becomes a complex of canonically-extended, fully decomposed automorphic vector bundles, placing us in the context of (3.2). For (4.1.7),

$$
i_{R}^{*}\left(\operatorname{Gr}_{F}^{p}\left(\Omega_{M_{\Sigma}}\left(\log Z_{\Sigma}\right) \otimes \widetilde{\mathcal{E}}^{\text {can }}\right)\right)
$$

becomes the restriction of the same to $Z_{\Sigma}(R)$.

We briefly recall the topological nerve spectral sequence that is of fundamental interest, which we treated in [HZ2, (3.5.4)]; it is the one associated to the local system $\widetilde{\boldsymbol{E}}$ and the covering of the Borel-Serre boundary by its closed faces, $\left\{\overline{e^{\prime}(P)}: P\right.$ maximal $\}$ :

$$
E_{1}^{r, s}=\bigoplus_{r(R)=r+1} H^{s}\left(\overline{e^{\prime}(R)}, \widetilde{\boldsymbol{E}}\right) \Longrightarrow H^{r+s}(\partial \bar{M}, \widetilde{\boldsymbol{E}}) .
$$

By $[\mathrm{HZ2},(3.5 .5)]$, the above coincides with the corresponding spectral sequence for deleted neighborhood cohomology on $M_{\Sigma}$, viz.,

$$
E_{1}^{r, s}=\bigoplus_{r(R)=r+1} H_{\mathrm{dn}}^{s}\left(Z_{\Sigma}(R), \widetilde{\boldsymbol{E}}\right) \Longrightarrow H_{\mathrm{dn}}^{r+s}\left(Z_{\Sigma}, \widetilde{\boldsymbol{E}}\right)
$$

We can use (4.1.7) and (4.1.8) to rewrite (4.1.16) in the form

$$
E_{1}^{r, s}=\bigoplus_{r(R)=r+1} \mathbb{H}^{s}\left(Z_{\Sigma}(R), i_{R}^{*} \mathcal{D} \mathcal{R}(M, \widetilde{\boldsymbol{E}})^{\mathrm{can}}\right) \Longrightarrow \mathbb{H}^{r+s}\left(Z_{\Sigma}, i^{*} \mathcal{D} \mathcal{R}(M, \widetilde{\boldsymbol{E}})^{\mathrm{can}}\right),
$$

where $\mathbb{H}$ indicates hypercohomology. This is a spectral sequence of mixed Hodge structures (see [HZ2, (5.5.2)]; cf. (4.5.2) here). A morphism of mixed Hodge structures is completely determined by its gradation for the Hodge filtration $F$, so we can grade for $F$ without losing information about kernels and images; this reduces such questions about the spectral sequence (4.1.12) to analogous considerations for spectral sequences 
of the form (3.2.1). Thus,

$$
\begin{aligned}
\operatorname{Gr}_{F}^{\bullet} E_{1}^{r, s}=\bigoplus_{r(R)=r+1} \operatorname{Gr}_{F}^{\bullet} \mathbb{H}^{s}\left(Z_{\Sigma}(R), i_{R}^{*} \mathcal{D} \mathcal{R}(M, \widetilde{\boldsymbol{E}})^{\mathrm{can}}\right) \\
\\
\Longrightarrow \operatorname{Gr}_{F}^{\bullet} \mathbb{H}^{r+s}\left(Z_{\Sigma}, i^{*} \mathcal{D} \mathcal{R}(M, \widetilde{\boldsymbol{E}})^{\mathrm{can}}\right) .
\end{aligned}
$$

By basic Hodge theory, we can write (4.1.18) as

$$
\begin{aligned}
\operatorname{Gr}_{F}^{\bullet} E_{1}^{r, s}=\bigoplus_{r(R)=r+1} \mathbb{H}^{s}\left(Z_{\Sigma}(R), \mathrm{Gr}_{F}^{\bullet}\right. & \left.\left\{i_{R}^{*} \mathcal{D} \mathcal{R}(M, \widetilde{\boldsymbol{E}})^{\mathrm{can}}\right\}\right) \\
& \Longrightarrow \mathbb{H}^{r+s}\left(Z_{\Sigma}, \operatorname{Gr}_{F}^{\bullet}\left\{i^{*} \mathcal{D R}(M, \widetilde{\boldsymbol{E}})^{\mathrm{can}}\right\}\right) .
\end{aligned}
$$

Using (4.1.14) and (4.1.16), we obtain:

$$
\begin{aligned}
\operatorname{Gr}_{F}^{\bullet} E_{1}^{r, s}=\bigoplus_{r(R)=r+1} H^{s}\left(Z_{\Sigma}(R), i_{R}^{*}\right. & \left.\left\{\operatorname{Gr}_{F}^{\bullet} \mathcal{H}^{\bullet}\left(\mathfrak{p}^{+}, E\right)\right\}^{\text {can }}\right) \\
& \Longrightarrow H^{r+s}\left(Z_{\Sigma}, i^{*}\left\{\operatorname{Gr}_{F}^{\bullet} \mathcal{H}^{\bullet}\left(\mathfrak{p}^{+}, E\right)\right\}^{\mathrm{can}}\right) .
\end{aligned}
$$

\subsection{The dual Bernstein-Gelfand-Gelfand complex}

Following Faltings $[\mathrm{F}]$, we can replace the de Rham complex $\mathcal{D} \mathcal{R}(M, \widetilde{\boldsymbol{E}})$ by a quasiisomorphic filtered subcomplex, the dual $B G G$ complex $\mathcal{B G} \mathcal{G}(M, \widetilde{\boldsymbol{E}})$, which is minimal in the sense that each degree is given by a sum of irreducible automorphic vector bundles. After taking cohomology, the quasi-isomorphism between $\mathcal{B G G}(M, \widetilde{\boldsymbol{E}})$ and $\mathcal{D R}(M, \widetilde{\boldsymbol{E}})$ recovers the results of [Z1]. Because its terms are irreducible automorphic vector bundles, the dual BGG complex has particularly nice rationality and integrality properties. Moreover, its cohomology is directly expressible in terms of automorphic forms.

We use the formalism introduced in $[\mathrm{H} 5, \S 2]$, but with slightly different notation. We assume first that $E$ is an absolutely irreducible representation of $G$, with highest weight $\Lambda \in \mathfrak{h}^{*}$ (see (0.3)); the irreducibility hypothesis will be removed at the end of the section. Let $W^{1}$ denote the set of Kostant representatives for the parabolic subalgebra $\mathfrak{P}^{+}$(see (0.3)). For any $t \in W^{1}$, we let $\Lambda(t)=t(\Lambda+\rho)-\rho$, where $\rho$ is the half-sum of positive roots. Then $\Lambda(t)$ is the highest weight of an irreducible representation of $K_{p}$, hence defines an irreducible automorphic vector bundle which we denote $\mathcal{B G G}^{t}(M, \widetilde{\boldsymbol{E}})$. For $0 \leqslant i \leqslant \operatorname{dim}_{\mathbb{C}}(M)$, let

$$
\mathcal{B G G}^{i}(M, \widetilde{\boldsymbol{E}})=\underset{\ell(t)=i}{\bigoplus} \mathcal{B G G}^{t}(M, \widetilde{\boldsymbol{E}})
$$

The $\mathcal{B G G}^{i}(M, \widetilde{\boldsymbol{E}})$ form a complex $\mathcal{B G G}(M, \widetilde{\boldsymbol{E}})$ in which the differentials are algebraic differential operators, and there is a canonical inclusion $[\mathrm{F}]$

$$
\mathcal{B G G}(M, \widetilde{\boldsymbol{E}}) \hookrightarrow \mathcal{D} \mathcal{R}(M, \widetilde{\boldsymbol{E}}) .
$$


In (4.2.1), one places the Hodge filtration $F$ on the left-hand side in the usual way for homogeneous vector bundles (see $[\mathrm{H} 5,(2.2 .3)]$ ), and we have likewise the Hodge filtration from (4.1) on the right-hand side.

THEOREM $4.2 .2([\mathrm{~F}])$. - The inclusion (4.2.1) is a filtered quasi-isomorphism that extends to a filtered quasi-isomorphism of complexes of canonical extensions.

REMARK. - It is implicit in the second part of the above theorem that the complex $\mathcal{B G G}(M, \widetilde{\boldsymbol{E}})^{\text {can }}$ is functorial with respect to change of toroidal compactification. This follows easily from Proposition 1.3.7, or alternatively from the functoriality of the canonical extension of the de Rham complex.

Theorem (4.2.2) yields immediately:

Corollary 4.2.3. - There is a spectral sequence

$$
E_{1}^{p, q}=H^{p+q}\left(\operatorname{Gr}_{F}^{p} \mathcal{B} \mathcal{G G}(M, \widetilde{\boldsymbol{E}})^{\mathrm{can}}\right) \Longrightarrow H^{p+q}(M, \widetilde{\boldsymbol{E}})
$$

that degenerates at $E_{1}$. The filtration induced by $F$ on the abutment is the Hodge filtration in its mixed Hodge structure.

Using (4.2.2) on both sides of (4.1.9), we obtain the following reformulation of the latter:

COROLlary 4.2.4. - In the situation of (4.1.9), there is a canonical isomorphism in the derived category of $\left(M_{P}\right)_{\Xi}$ :

$$
R\left(\bar{\pi}_{P}\right)_{*}\left(i_{R}^{*}\right)\{\mathcal{B G} \mathcal{G}(M, \widetilde{\boldsymbol{E}})\}^{\text {can }} \approx\left\{\mathcal{B G} \mathcal{G}\left(M_{P}, \widetilde{\boldsymbol{C}}^{\bullet}\left(\Gamma_{\ell, R}, H^{\bullet}\left(\mathfrak{w}_{P}, E\right)\right)\right)\right\}^{\text {can }} .
$$

We present below a more concrete way to see this quasi-isomorphism.

We begin with some general considerations involving Weyl group combinatorics. Let $G$ be a reductive group as in (0.2), with chosen set $\Phi^{+}$of positive roots, and let $P, Q \subset G$ denote two standard parabolics; let $R=P \cap Q$. Let $\mathfrak{w}_{P}, \mathfrak{w}_{Q}, \mathfrak{w}_{R}$ denote the Lie algebras of the unipotent radicals of $P, Q$, and $R$, respectively; similarly, let $L_{\text {? }}$, for ? $=P, Q, R$, be a compatible set of Levi factors. Then we can decompose

$$
\mathfrak{w}_{R}=\mathfrak{w}_{P Q} \oplus \mathfrak{w}_{P}^{Q} \oplus \mathfrak{w}_{Q}^{P}
$$

in such a way that

$$
\mathfrak{w}_{P}=\mathfrak{w}_{P Q} \oplus \mathfrak{w}_{P}^{Q}, \quad \mathfrak{w}_{Q}=\mathfrak{w}_{P Q} \oplus \mathfrak{w}_{Q}^{P}
$$

( so $\mathfrak{w}_{P Q}=\mathfrak{w}_{P} \cap \mathfrak{w}_{Q}$ ) and $\Phi_{R}=\Phi_{P Q} \sqcup \Phi_{P}^{Q} \sqcup \Phi_{Q}^{P}$; here $\Phi_{R}, \Phi_{P Q}, \Phi_{P}^{Q}, \Phi_{Q}^{P}$ denote the sets of positive roots in $\mathfrak{w}_{R}$, etc. Let $W^{?} \subset W$, for $?=P, Q, R$, denote the corresponding sets of Kostant representatives, and let $W_{\text {? }} \subset W$ be the Weyl group of $L_{\text {? }}$. Finally, $L_{P} \cap R=L_{P} \cap Q$ is parabolic in $L_{P}$ and we let $W_{P}^{R}=W_{P}^{Q}$ denote the Kostant representatives in $W_{L_{P}}=W_{P}$ relative to $W_{L_{P} \cap R}$; define $W_{Q}^{R}=W_{Q}^{P}$ similarly. As in Lemma 3.1.6, we have canonical decompositions

$$
W^{R}=W_{P}^{R} \cdot W^{P}=W_{Q}^{R} \cdot W^{Q} .
$$


We denote the elements of $W^{R}$ by $\omega$, and those of $W_{P}^{Q}, W^{P}, W_{Q}^{P}, W^{Q}$ by $a, b, s$, and $t$, respectively. For any $\omega \in W^{R}$ we write

$$
\Phi_{P Q}(\omega)=\omega\left(\Phi^{+}\right) \cap-\Phi_{P Q} ; \Phi_{P}^{Q}(\omega)=\omega\left(\Phi^{+}\right) \cap-\Phi_{P}^{Q} ; \Phi_{Q}^{P}(\omega)=\omega\left(\Phi^{+}\right) \cap-\Phi_{Q}^{P} .
$$

Then

$$
\ell(\omega)=\left|\Phi_{P Q}(\omega)\right|+\left|\Phi_{P}^{Q}(\omega)\right|+\left|\Phi_{Q}^{P}(\omega)\right| .
$$

If $\omega=a b=s t$ by the factorizations of (4.2.6), we then have

$$
\begin{gathered}
\ell(\omega)=\ell(a)+\ell(b)=\ell(s)+\ell(t), \\
\ell(a)=\left|\Phi_{P}^{Q}(\omega)\right|, \quad \ell(b)=\left|\Phi_{P Q}(\omega)\right|+\left|\Phi_{Q}^{P}(\omega)\right| ; \\
\ell(s)=\left|\Phi_{Q}^{P}(\omega)\right|, \quad \ell(t)=\left|\Phi_{P Q}(\omega)\right|+\left|\Phi_{P}^{Q}(\omega)\right| .
\end{gathered}
$$

Finally, if $\omega, \omega^{\prime} \in W$, we write $\omega \rightarrow \omega^{\prime}$ if there exists $\gamma \in \Phi^{+}$such that $\omega=r_{\gamma} \omega^{\prime}$ and $\ell(\omega)=\ell\left(\omega^{\prime}\right)+1$; here $r_{\gamma}$ is the reflection attached to $\gamma$.

Lemma 4.2.10. - Let $\omega, \omega^{\prime} \in W^{R}$, with factorizations $\omega=a b=s t, \omega^{\prime}=a^{\prime} b^{\prime}=s^{\prime} t^{\prime}$ as above. Suppose $\omega \rightarrow \omega^{\prime}$. Suppose further that $b=b^{\prime}$. Then $s=s^{\prime}$ and $t \rightarrow t^{\prime}$.

Proof. - It follows from (4.2.8) and (4.2.9) that $\ell(t)=\ell\left(t^{\prime}\right)+1$. Indeed, $\Phi_{P Q}(\omega)=$ $\Phi_{P Q}(b)=\Phi_{P Q}\left(\omega^{\prime}\right)$. Suppose we know that $s=s^{\prime}$. Writing $s t=r_{\gamma} s t^{\prime}$, we see that $t=r_{s^{-1} \gamma} t^{\prime}$, hence $t \rightarrow t^{\prime}$. So it remains to prove $s=s^{\prime}$. For this it suffices to show that $\Phi_{P}^{Q}(s)=\Phi_{P}^{Q}\left(s^{\prime}\right)$, since both $s$ and $s^{\prime}$ preserve $\mathfrak{w}_{Q}$. But

$$
\Phi_{P}^{Q}(s)=\Phi_{P}^{Q}(\omega)=\Phi_{P}^{Q}(b), \quad \Phi_{P}^{Q}\left(s^{\prime}\right)=\Phi_{P}^{Q}\left(\omega^{\prime}\right)=\Phi_{P}^{Q}\left(b^{\prime}\right),
$$

where the first equalities follow from the definition of $W^{Q}$ and the second from the fact that $W_{P}^{Q}$ preserves $\mathfrak{w}_{P}$. The assertion is now clear.

Let $\mathfrak{g}, \mathfrak{p}, \mathfrak{q}, \mathfrak{r}$ denote the Lie algebras of $G, P, Q$, and $R$ respectively. Letting $E$ and $\Lambda$ be as above, we consider the generalized Bernstein-Gelfand-Gelfand resolutions of $E$ with respect to the parabolic subalgebras we have introduced. Thus for $\omega \in W^{R}$ let

$$
\mathbb{M}_{R}(\Lambda(\omega))=\mathfrak{U}(\mathfrak{g}) \otimes_{\mathfrak{U}(\mathfrak{r})} E(\Lambda(\omega)),
$$

with $\Lambda(\omega)$ defined as above and $E(\Lambda(\omega))$ the finite-dimensional $L_{R}$ module with highest weight $\Lambda(\omega)$, extended as usual to an irreducible $\mathfrak{U}(\mathfrak{r})$ module. The generalized $B G G$ complex $B G G^{R}(E)$ of $E$, relative to $\mathfrak{r}$, is the complex

$$
\begin{aligned}
& \cdots \longrightarrow \bigoplus_{\omega \in W^{R}, \ell(w)=i+1} \mathbb{M}_{R}(\Lambda(\omega)) \quad \stackrel{d_{i}^{R}}{\longrightarrow} \underset{\omega \in W^{R}, \ell(\omega)=i}{\bigoplus} \mathbb{M}_{R}(\Lambda(w)) \longrightarrow \\
& \cdots \longrightarrow \mathbb{M}_{R}(\Lambda) \longrightarrow 0 .
\end{aligned}
$$

Here $d_{i}^{R}$ is the differential constructed in [Le, $\S 4$ ]. It can be written as a sum

$$
d_{i}^{R}=\sum_{\omega, \omega^{\prime} \in W^{R}, \omega \longrightarrow \omega^{\prime}, \ell\left(\omega^{\prime}\right)=i} d_{\omega, \omega^{\prime}}^{R},
$$


where $d_{\omega, \omega^{\prime}}^{R}: \mathbb{M}_{R}(\Lambda(\omega)) \rightarrow \mathbb{M}_{R}\left(\Lambda\left(\omega^{\prime}\right)\right)$. These differentials can be defined uniquely up to sign ([RC, Lemma 10.5]), and the natural surjection $\mathbb{M}_{R}(\Lambda) \rightarrow E$ (defined up to scalar multiples) defines a quasi-isomorphism $B G G^{R}(E) \approx E$ in the category of $\mathfrak{U}(\mathfrak{g})$-modules. It follows from the results of [RC, $\S \S 10-11]$ that changing the sign of one differential changes all signs simultaneously, and that the different choices of sign yield isomorphic complexes.

Let $\bar{P}=L_{P} \bar{N}_{P}$ be the parabolic opposite to $P$, with Lie algebra $\overline{\mathfrak{p}}=\mathfrak{l} \oplus \overline{\mathfrak{w}}_{P}$. As $\mathfrak{U}(\overline{\mathfrak{p}})$-module we have $\mathbb{M}_{R}(\Lambda(\omega))=\mathfrak{U}(\overline{\mathfrak{p}}) \otimes_{\mathfrak{U}\left(\mathfrak{I}_{P} \cap \mathfrak{r}\right)} E(\Lambda(\omega))$. For any irreducible finite-dimensional $L_{P}$-module $V$, we let $B G G^{L_{P} \cap R}(V)$ denote the generalized BGG resolution of $V$ relative to $L_{P} \cap R$, and define the maps $d_{a, a^{\prime}}^{L_{P} \cap R}$ by analogy with (4.2.13).

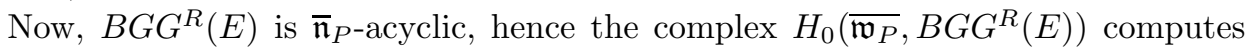
$H_{\bullet}\left(\mathfrak{w}_{P}, E\right)$, via the quasi-isomorphism $B G G^{R}(E) \approx E$. For any $\omega$ we have natural isomorphisms

$$
H_{0}\left(\overline{\mathfrak{w}}_{P}, \mathbb{M}_{R}(\Lambda(\omega))\right) \stackrel{\sim}{\longrightarrow} \mathfrak{U}\left(\mathfrak{l}_{P}\right) \otimes_{\mathfrak{U}\left(\mathfrak{l}_{P} \cap \mathfrak{r}\right)} E(\Lambda(\omega)) .
$$

We let $\mathbb{M}\left(\Lambda(\omega) ; L_{P}\right)$ denote the right-hand side of (4.2.14). Then

Proposition 4.2.15

(i) There is a canonical isomorphism of complexes

$$
H_{0}\left(\overline{\mathfrak{n}}_{P}, B G G^{R}(E)\right) \stackrel{\sim}{\longrightarrow} \bigoplus_{b \in W^{P}} B G G^{R(P)}(E(\Lambda(b))[\ell(b)] .
$$

Here $E(\Lambda(b))$ is the irreducible representation of $L_{P}$ with highest weight $\Lambda(b)$.

(ii) More precisely, for any pair $a, a^{\prime} \in W_{P}^{Q}=W^{R(P)}$, with $a \rightarrow a^{\prime}$, and for any $b \in W^{P}$, there is a pair $t, t^{\prime} \in W^{Q}$ and $s \in W_{Q}^{P}$ such that the differential $d_{a, a^{\prime}}^{R(P)}$ in $B G G^{R(P)}(E(\Lambda(b)))[\ell(b)]$ is obtained by applying $H_{0}\left(\overline{\mathfrak{n}}_{P}, \bullet\right)$ to $d_{\omega, \omega^{\prime}}^{R}$, with $\omega=a b=s t$, $\omega^{\prime}=a^{\prime} b=s t^{\prime}$.

(iii) For any pair $\omega, \omega^{\prime} \in W^{R}$ not obtained as in (ii), the morphism $H_{0}\left(\overline{\mathfrak{n}}_{P}, d_{\omega, \omega^{\prime}}^{R}\right)$ vanishes.

Proof. - We first verify (ii) and (iii). Assertion (ii) is a consequence of Lemma 4.2.10. Suppose $\omega \rightarrow \omega^{\prime}$ in $W^{R}$, and write $\omega=a b, \omega^{\prime}=a^{\prime} b^{\prime}$. Now it follows from the theory of Verma modules for $L_{P}$ that the infinitesimal character separates $\mathbb{M}\left(\Lambda(\omega) ; L_{P}\right)$ from $\mathbb{M}\left(\Lambda\left(\omega^{\prime}\right) ; L_{P}\right)$ if $b \neq b^{\prime}$. Thus $H_{0}\left(\overline{\mathfrak{n}_{P}}, d_{\omega, \omega^{\prime}}^{R}\right)=0$ unless $b=b^{\prime}$, and (iii) follows from (ii).

The assertion (i) follows easily from (ii) and (iii) by checking degrees. Indeed, by writing $\omega=a b$ as above, we see that the assertion is true in each degree separately, and it remains only to check that the differentials correspond (up to sign). But this follows from the explicit construction of the generalized BGG resolutions in [Le,RC].

We fix a toroidal compactification $\operatorname{Sh}(G, X)_{\Sigma}$ (omitting the left subscript for the level subgroup), with $\Sigma$ assumed to be sufficiently fine for the following arguments. 
Fix a maximal parabolic subgroup $P \subset G$. Let $P^{\prime} \subset P$ denote $G_{h, P} \cdot W_{P}$, as in [HZ1, 1.2.3]. Thus there is a mixed Shimura datum $\left(P^{\prime}, X\right)$ and a partially compactified mixed Shimura variety $\operatorname{Sh}\left(P^{\prime}, X\right)_{\Sigma^{\prime}}$ corresponding to the $P$-stratum of the boundary of $\operatorname{Sh}(G, X)_{\Sigma}$. We refer to (1.4) for the morphisms $\pi_{1, \Sigma^{\prime}}, \pi_{2, \Sigma^{\prime}}$, and let $\pi_{\Sigma^{\prime}}=\pi_{1, \Sigma^{\prime}} \circ \pi_{2, \Sigma^{\prime}}$ denote the composite morphism from $\operatorname{Sh}\left(P^{\prime}, \mathcal{X}\right)_{\Sigma^{\prime}}$ to $M_{P, \Sigma(P)}$, where $M_{P, \Sigma(P)}$ is a toroidal compactification of the pure Shimura variety associated to $\left(G_{h, P}, W \backslash X\right)$. We let $i_{P}^{o}$ denote the inclusion of the open $P$-stratum in the boundary, and let $\pi: \operatorname{Sh}\left(P^{\prime}, \mathcal{X}\right) \rightarrow M_{P}$ be the corresponding map of uncompactified (mixed) Shimura varieties. The differentials in the complex $i_{P}^{o, *} \mathcal{B G} \mathcal{G}(M, \widetilde{\boldsymbol{E}}$ ) (with $M$ a connected component of $\operatorname{Sh}(G, X))$ are differential operators of positive degree, hence not $\mathcal{O}$-linear. But they are morphisms of abelian sheaves, hence give rise to functorial morphisms under $R^{i} \pi_{*}$, for each $i$, and these are also differential operators. Our goal is to calculate these directly at the level of $\mathcal{B G G}\left(M_{P}, \widetilde{\boldsymbol{C}}^{\bullet}\left(\Gamma_{\ell, R}, H^{\bullet}\left(\mathfrak{w}_{P}, E\right)\right)\right)$, using Proposition 4.2.4.

We apologize that the standard maximal parabolic $P$ here will play the role of $\bar{P}$ in the applications of Proposition 4.2.15 in what follows; this is because we are ultimately interested in homology and cohomology of $\mathfrak{w}_{P}$, not $\overline{\mathfrak{w}}_{P}$. Thus, our notation is correspondingly contorted. First, let $Q_{1}$ be the complex parabolic with Lie algebra $\mathfrak{k} \oplus \mathfrak{P}^{-}$, and $\bar{Q}=\operatorname{Ad}\left(c_{P}^{-1}\right)\left(Q_{1}\right)$. Then, let $\bar{Q} \subset G$ denote the standard complex parabolic conjugate to $Q_{1}=\mathfrak{k} \oplus \mathfrak{P}^{-}$; and $Q$ the parabolic opposite to $\bar{Q}$, viz., $\operatorname{Ad}\left(c_{P}\right)\left(\mathfrak{k} \oplus \mathfrak{P}^{-}\right)$. Let $R=\bar{P} \cap Q$, and $R_{1}=\operatorname{Ad}\left(c_{P}\right) R \subset Q_{1}$.

Via $\operatorname{Ad}\left(c_{P}\right)$ we may identify $W^{Q}=W^{Q_{1}}, W^{R}=W^{R_{1}}$. We use the same letters $a, b, s, t$ as above to denote corresponding Kostant representatives. In particular, we denote the components of the BGG complex by $\mathcal{B G} \mathcal{G}^{t}(\widetilde{\boldsymbol{E}})$, dropping the " $M$ " in (4.2.1). Similarly, for each $q$ and $t$, we have

$$
R^{q} \pi_{*} \mathcal{B G}^{t}(\widetilde{\boldsymbol{E}}) \stackrel{\sim}{\longrightarrow} \bigoplus_{s \in W_{Q}^{\bar{P}}, \ell(s)=q}\left[\mathcal{B G}^{t}(\widetilde{\boldsymbol{E}})\right]^{s} .
$$

Here if $\mathcal{B G G}^{t}(\widetilde{\boldsymbol{E}})$ is the automorphic vector bundle associated to the representation $E(t)$ of $K_{p},\left[\mathcal{B G G}^{t}(\widetilde{\boldsymbol{E}})\right]^{s}$ is the automorphic vector bundle on $M_{P}$ associated to $\boldsymbol{C}^{\bullet}\left(\Gamma_{\ell, P}, H^{s}\left(\mathfrak{s}_{P}^{\prime}, E(t)\right)\right)$, where $H^{s}$ denotes the Kostant constituent of the cohomology associated to $s \in W_{Q}^{\bar{P}}$. We are abusing notation (in a harmless way) by writing in the discrete group $\Gamma_{\ell, P}$ in this adelic situation. If now $\omega=s t=a b$ as in (4.2.6), then we can identify

$$
\left[\mathcal{B G G}^{t}(\widetilde{\boldsymbol{E}})\right]^{s}=\boldsymbol{C}^{\bullet}\left(\Gamma_{\ell, P}, \mathcal{B} \mathcal{G} \mathcal{G}^{a}\left(\widetilde{\boldsymbol{H}}^{b}\left(\mathfrak{w}_{P}, E\right)\right)\right)
$$

It then follows from (4.2.6) that

LEMma 4.2.18. - The identifications (4.2.16) and (4.2.17) define an isomorphism between the constituents of the two quasi-isomorphic complexes in (4.2.4). 
It remains to show that these isomorphisms are compatible with the differentials on the two sides of (4.2.4). Recall that, if $\mathcal{E}$ and $\mathcal{F}$ are vector bundles on a smooth complex variety $Z$, a $\mathbb{C}$-linear map $\Delta: \mathcal{E} \rightarrow \mathcal{F}$ is a differential operator of order $n$ if it can be factored as

$$
\mathcal{E} \stackrel{j^{n}}{\longrightarrow} \operatorname{Jet}^{n}(\mathcal{E}) \longrightarrow \mathcal{F},
$$

where $\operatorname{Jet}^{n}(\mathcal{E})$ is the bundle of $n$-jets of sections of $\mathcal{E}, j^{n}$ is the canonical differential operator, and the right-hand arrow is $\mathcal{O}_{Z}$-linear. (Here the notation for jets is as in [H1].) Classification of differential operators $\Delta: \mathcal{E} \rightarrow \mathcal{F}$ thus comes down to classification of $\mathcal{O}_{Z}$-linear maps $\operatorname{Jet}^{\infty}(\mathcal{E}) \rightarrow \operatorname{Jet}^{\infty}(\mathcal{F})$, where $\mathrm{Jet}^{\infty}$ denotes the inverse limit over the jet bundles of finite order.

For our purposes, it is more useful to look at the dual picture. Let $\mathcal{D}_{Z}$ denote the sheaf of differential operators on $Z$; we write $\mathcal{D}$ instead of $\mathcal{D}_{Z}$ when the base $Z$ is understood. For $\mathcal{G}$ a vector bundle on $Z$, let $\mathcal{D}(\mathcal{G})=\mathcal{D}_{Z}(\mathcal{G})$ denote the locally free $\mathcal{D}$-module $\mathcal{D} \otimes \mathcal{O}_{Z} \mathcal{G}^{\vee}$. Then to $\Delta$ as above we associate a homomorphism of $\mathcal{D}$-modules

$$
\Delta^{\vee}: \mathcal{D}\left(\mathcal{F}^{\vee}\right) \longrightarrow \mathcal{D}\left(\mathcal{E}^{\vee}\right)
$$

More precisely, $\operatorname{Jet}^{\infty}(\mathcal{E})$ is naturally isomorphic to the $\mathcal{O}_{Z}$-linear dual of $\mathcal{D}_{Z}\left(\mathcal{E}^{\vee}\right)$ and the dual of $\Delta^{\vee}$ is the map of infinite jet bundles mentioned above. We call $\Delta^{\vee}$ the linearization of $\Delta$.

We fix a point $p \in X$ compatible with our system of standard rational parabolic subgroups. If $[W]$ is the automorphic vector bundle corresponding to the irreducible representation $\left(\rho, W_{\rho}\right)$ of the stabilizer $K_{p}$ of $p$, then $\mathcal{D}([W])$ is the automorphic vector bundle corresponding to the natural representation of $\mathfrak{P}_{p}^{-}$on the generalized Verma module

$$
\mathbb{D}\left(W_{\rho}^{*}\right)=\mathfrak{U}(\mathfrak{g}) \otimes_{\mathfrak{U}\left(\mathfrak{P}_{p}^{-}\right)} W_{\rho}^{*} .
$$

We point out that $\mathcal{D}\left([W]^{\text {can }}\right) \neq \mathcal{D}([W])^{\text {can }}$. However, we will only be concerned with the latter, to which logarithmic differential operators naturally extend.

We now fix a maximal standard rational parabolic $P$ and the subgroup $P^{\prime} \subset$ $P$ to which the corresponding boundary mixed Shimura datum $\left(P^{\prime}, X\right)$ is attached. The constructions in (1.2) associate automorphic vector bundles on the associated mixed Shimura varieties to representations of the stabilizer in $P^{\prime}$ of the fixed point $p \in X$. This stabilizer will be denoted $F^{0} P^{\prime}$, since its Lie algebra is $F^{0} \operatorname{Lie}\left(P^{\prime}\right)$ for the Hodge filtration associated to $p$; in particular, we drop the base point $p$ from the notation. In what follows, we will identify automorphic vector bundles with the locally homogeneous vector bundles attached to representations of

$$
F^{0} \operatorname{Lie}\left(P^{\prime}\right)=\mathfrak{k}_{h, P} \oplus \mathfrak{p}_{h, P}^{-} \oplus \mathfrak{v}^{-},
$$

and we will also drop the subscript $P$ from the above.

Without loss of generality we may assume that, for fixed $x$ in the $P$-stratum, there is an étale analytic morphism from $\operatorname{Sh}\left(P^{\prime}, X\right)_{\Sigma^{\prime}}$ to a neighborhood of $x$. The 
pullback of $\mathcal{D}([W])$ to $\operatorname{Sh}\left(P^{\prime}, X\right)$ via this morphism is the $\mathcal{D}(\bullet)$ of the pullback of $[W]$. This can be determined just as above, and we find that it is the automorphic vector bundle corresponding to the natural representation of $F^{0} \mathrm{Lie}\left(P^{\prime}\right)$ on the tensor product module

$$
\mathbb{D}_{P}\left(W_{\rho}^{*}\right)=\mathfrak{U}\left(\operatorname{Lie}\left(P^{\prime}\right)\right) \otimes \mathfrak{U}\left(F^{0} \operatorname{Lie}\left(P^{\prime}\right)\right) W_{\rho}^{*} \stackrel{\sim}{\longrightarrow} \mathfrak{U}(\operatorname{Lie}(P)) \otimes_{\mathfrak{U}\left(F^{0} \operatorname{Lie}\left(P^{\prime}\right) \oplus \mathfrak{g}_{\ell}\right)} W_{\rho}^{*}
$$

where the second isomorphism just serves to indicate that arithmetic subgroups of $G_{\ell}=G_{\ell, P}$ act on the coefficients and commute with all homogeneous differential operators.

Now we concentrate on the map, again denoted $\pi: Z=\operatorname{Sh}\left(P^{\prime}, X\right)_{\Sigma^{\prime}} \rightarrow M_{P}$, where the subscript $\Sigma^{\prime}$ corresponds to a compactification in the vertical direction. In particular the map $\pi$ is smooth and proper. Henceforward, we let

$$
\mathcal{E}=\mathcal{B G}^{t^{\prime}}(\widetilde{\boldsymbol{E}}), \quad \mathcal{F}=\mathcal{B G}^{t}(\widetilde{\boldsymbol{E}})
$$

with $t \rightarrow t^{\prime}$ in $W^{Q}$ as above. Let $\Lambda$ be the highest weight of the contragredient of $E$. Then the fibers at $p$ of $\mathcal{F}^{\vee}$ and $\mathcal{E}^{\vee}$ are $\mathbb{D}_{P}\left(H^{t}\left(\mathfrak{p}^{+}, E\right)^{\vee}\right)$ and $\mathbb{D}_{P}\left(H^{t^{\prime}}\left(\mathfrak{p}^{+}, E\right)^{\vee}\right)$, respectively. In turn, these can be identified respectively with $\mathbb{M}_{Q_{1}}(\Lambda(t))$ and $\mathbb{M}_{Q_{1}}\left(\Lambda\left(t^{\prime}\right)\right)$. The differential $d_{t, t^{\prime}}^{Q_{1}}: \mathbb{M}_{Q_{1}}(\Lambda(t)) \rightarrow \mathbb{M}_{Q_{1}}\left(\Lambda\left(t^{\prime}\right)\right)$ in the generalized $B G G$ complex gives rise to the differential operator $\Delta_{t, t^{\prime}}: \mathcal{E} \rightarrow \mathcal{F}$, corresponding to a component of the differential in $\mathcal{B G} \mathcal{G}(\widetilde{\boldsymbol{E}})$.

To determine the image of $\Delta_{t, t^{\prime}}$ under $R^{i} \pi_{*}$, we pass to linearizations. The map $\Delta_{t, t^{\prime}}^{\vee}$ of (4.2.19) is a morphism of $\mathcal{D}_{Z}$-modules, hence it is natural to consider its image under the direct image in the category of $\mathcal{D}_{Z}$-modules. Following [B4], we denote this derived functor $R \pi_{+}$.

Proposition 4.2.21

(i) Let $\mathcal{G}=\mathcal{E}$ or $\mathcal{F}$, and $d=\operatorname{dim} Z-\operatorname{dim} M$. For each $q \geqslant 0$, there is a natural isomorphism

$$
R^{\bullet} \pi_{+} \mathcal{D}_{Z}\left(\mathcal{G}^{\vee}\right) \stackrel{\sim}{\longrightarrow} \mathcal{D}_{M_{P}}\left(R^{d-\bullet} \pi_{*}\left(\mathcal{G}^{\vee}\right)\right) .
$$

(ii) Under this isomorphism, the image of $\Delta_{t, t^{\prime}}^{\vee}$ is the linearization of the image of $\Delta_{t, t^{\prime}}$ via $R^{d-\bullet} \pi_{*}$.

Proof. - The first assertion is a simple consequence of the definitions. Recall that $\pi$ is smooth and proper. By definition, for any $\mathcal{D}_{Z}$-module $\mathcal{N}$,

$$
R \pi_{+} \mathcal{N}=R \pi_{*}\left(\mathcal{D}_{\left\{M_{P} \leftarrow Z\right\}} \underset{\mathcal{D}_{Z}}{\stackrel{\mathbb{L}}{\otimes}} \mathcal{N}\right)
$$

Here $\mathcal{D}_{\left\{M_{P} \leftarrow Z\right\}}=\pi^{*}\left(\mathcal{D}_{M_{P}}\right) \otimes \omega_{Z / M_{P}}$, where $\pi^{*}$ is inverse image in the category of $\mathcal{O}$-modules and $\omega_{Z / M_{P}}=\Omega_{Z / M_{P}}^{d}$ is the relative dualizing sheaf. In our case, $N=\mathcal{D}_{Z} \otimes_{\mathcal{O}_{Z}} \mathcal{G}^{\vee}$ is a free $\mathcal{D}_{Z}$-module, hence we can replace $\otimes^{\mathbb{L}}$ by $\otimes$ above, and we 
have

$$
\begin{aligned}
R^{\bullet} \pi_{+} \mathcal{D}_{Z}\left(\mathcal{G}^{\vee}\right) & =R^{\bullet} \pi_{*}\left[\pi^{*}\left(\mathcal{D}_{M_{P}}\right) \otimes \omega_{Z / M_{P}} \otimes \mathcal{G}^{\vee}\right] \\
& \stackrel{\sim}{\longrightarrow} \mathcal{D}_{M_{P}} \otimes R^{\bullet} \pi_{*}\left(\omega_{Z / M_{P}} \otimes \mathcal{G}^{\vee}\right) \\
& \stackrel{\sim}{\longrightarrow} \mathcal{D}_{M_{P}} \otimes\left(R^{\bullet} \pi_{*} \mathcal{G}\right)^{\vee}=\mathcal{D}_{M_{P}}\left(R^{d-\bullet} \pi_{*}\left(\mathcal{G}^{\vee}\right)\right)
\end{aligned}
$$

where the first isomorphism is the projection formula and the second is Serre duality. The second assertion is well-known (see [Sa1, Lemme 2.3.6]).

We rewrite the formulas (4.2.22) in terms of representation theory. Recall that

$$
\mathfrak{w}_{P, \mathbb{C}}=\mathfrak{u}_{\mathbb{C}} \oplus \mathfrak{v}^{+} \oplus \mathfrak{v}^{-}
$$

as modules over $\mathfrak{k}_{h}$; here $\mathfrak{u}=\operatorname{Lie}(U)$ with $U$ the center of $W_{P}$, as usual. We write $\mathfrak{w}^{+}=$ $\mathfrak{u}_{\mathbb{C}} \oplus \mathfrak{v}_{p}^{+}$. Then the bundle $\omega_{Z / M_{P}}$ is the automorphic vector bundle on $M_{P}$ associated to the adjoint representation of $\mathfrak{k}_{h}$ on the highest exterior power of the holomorphic cotangent space at the image of $p$ in $M_{P}$, i.e. on $\wedge^{d}\left(\mathfrak{w}^{+}\right)^{*}$. Next, $\pi^{*}\left(\mathcal{D}_{M_{P}}\right)$ is the bundle associated to the adjoint representation of $F^{0} \operatorname{Lie}\left(P^{\prime}\right)$ (or $F^{0} \operatorname{Lie}(P)$, if we want to keep track of the $G_{\ell}$-action) on $H_{0}\left(\mathfrak{w}^{+}, \mathfrak{U}\left(\operatorname{Lie}\left(P^{\prime}\right)\right)\right)\left(\right.$ or $H_{0}\left(\mathfrak{w}^{+}, \mathfrak{U}(\operatorname{Lie}(P))\right)$, but the addition of $\mathfrak{g}_{\ell}$ at this point changes nothing in the final calculation). Finally, the functor $R \pi_{*}$ corresponds to the standard bar complex $C^{\bullet}\left(\mathfrak{v}^{-}, \bullet\right)$ for Lie algebra cohomology. Thus the first line of (4.2.22) expresses $R \pi_{+} \mathcal{D}_{Z}\left(\mathcal{G}^{\vee}\right)$ as the automorphic vector bundle attached to

$$
C^{\bullet}\left(\mathfrak{v}^{-}, H_{0}\left(\mathfrak{w}^{+}, \mathfrak{U}(\operatorname{Lie}(P)) \otimes_{\mathfrak{U}\left(F^{0} \operatorname{Lie}(P)\right)} \wedge^{d}\left(\mathfrak{w}^{+}\right)^{*} \otimes W\right)\right),
$$

where $\mathcal{G}^{\vee}$ is the automorphic vector bundle attached to the representation of $\mathfrak{k}$ on $W$. Since $\mathfrak{v}^{-} \subset F^{0} \operatorname{Lie}\left(P^{\prime}\right)$ the action passes across the tensor product to yield

$$
H_{0}\left(\mathfrak{w}^{+}, \mathfrak{U}(\operatorname{Lie}(P)) \otimes_{\mathfrak{U}\left(F^{0} \operatorname{Lie}(P)\right)} C^{\bullet}\left(\mathfrak{v}^{-}, \wedge^{d}\left(\mathfrak{w}^{+}\right)^{*} \otimes W\right)\right) .
$$

Koszul duality [Kn, Theorem $6.10(6.30)]$ provides a canonical identification

$$
C^{\bullet}\left(\mathfrak{v}^{-}, ?\right) \stackrel{\sim}{\longrightarrow} C_{d_{-}-\bullet}\left(\mathfrak{v}^{-}, \wedge^{\operatorname{dim} \mathfrak{v}^{-}}\left(\mathfrak{v}^{-}\right)^{*} \otimes ?\right),
$$

where $C_{\bullet}\left(\mathfrak{v}^{-}, ?\right)$ is the standard complex for Lie algebra homology and $d_{-}=\operatorname{dim} \mathfrak{v}^{-}$. Thus we can replace the last complex by

$$
\begin{aligned}
& H_{0}\left(\mathfrak{w}^{+}, \mathfrak{U}\left(\operatorname{Lie}\left(P^{\prime} \cdot W_{2} P_{\mathbb{C}}^{\prime}\right) \otimes_{\mathfrak{U}\left(F^{0} \operatorname{Lie}\left(P^{\prime}\right)\right)} C_{d_{-}-\bullet}\left(\mathfrak{v}^{-}, \wedge^{d_{P}}\left(\mathfrak{w}_{P}\right)^{*} \otimes W\right)\right)\right. \\
& \quad=H_{0}\left(\mathfrak{w}^{+}, \mathfrak{U}\left(\operatorname{Lie}\left(P^{\prime} \cdot W_{2} P_{\mathbb{C}}^{\prime}\right) \otimes_{\mathfrak{U}\left(F^{0} \operatorname{Lie}\left(P^{\prime}\right)\right)} \wedge^{d_{P}}\left(\mathfrak{w}_{P}\right)^{*} \otimes C_{d_{--} \bullet}\left(\mathfrak{v}^{-}, W\right)\right)\right.
\end{aligned}
$$

Here $d_{P}=\operatorname{dim} \mathfrak{w}_{P}$ and we have used (4.2.23) and the fact that $\mathfrak{v}^{-}$, being unipotent, has trivial determinant on $\mathfrak{w}_{P}$.

Remember that $\mathfrak{v}^{-}$acts on $W$ via an identification with $\mathfrak{s} \subset \mathfrak{k}$, the unipotent radical of a parabolic subalgebra we will denote $\overline{\mathfrak{r}}(Q)$. Similarly, we identify $\mathfrak{g}_{\ell}$ with $\operatorname{Ad}\left(c_{P}\right)\left(\mathfrak{g}_{\ell}\right) \subset \mathfrak{k}$. Then there is a Levi decomposition

$$
\overline{\mathfrak{r}}(Q)=\mathfrak{k}_{h} \oplus \mathfrak{g}_{\ell} \oplus \mathfrak{s}
$$


and we let $\mathfrak{r}(Q) \subset \mathfrak{k}$ denote the opposite parabolic, $R(Q)$ the corresponding parabolic subgroup. We can replace $W$ as $\mathfrak{U}(\mathfrak{k})$-module by the quasi-isomorphic complex $B G G^{R(Q)}(W)$. Then there is a canonical quasi-isomorphism

$$
\begin{aligned}
C_{\bullet}(\mathfrak{s}, W) & \cong H_{0}\left(\mathfrak{s}, B G G_{\bullet}^{R(Q)}(W)\right) \\
& \stackrel{\sim}{\longrightarrow} H_{0}\left(\mathfrak{s}, \underset{\ell(s)=\bullet}{\bigoplus} \mathfrak{U}(\mathfrak{k}) \otimes_{\mathfrak{U}(\mathfrak{r}(Q))} E(\Lambda(W)(s))\right) .
\end{aligned}
$$

Here $s$ runs through the set of Kostant representatives relative to $R(Q)$, as in the first part of this section, and we use $\Lambda(W)$ to designate a highest weight of $W$.

We now return to the notation $\mathcal{E}, \mathcal{F}$, associated to the pair $t \rightarrow t^{\prime} \in W^{Q}$; i.e., associated respectively to the representations $E\left(t^{\prime}\right)$ and $E(t)$ of $\mathfrak{k}$. We extend $\mathfrak{U}(\mathfrak{k}) \otimes_{\mathfrak{U}(\mathfrak{r}(Q))}$ $E(t)^{*}(s)$ trivially to a module over $\mathfrak{U}\left(\mathfrak{k} \oplus \mathfrak{p}^{-}\right)$and then restrict to $\mathfrak{U}\left(\mathfrak{k}_{h} \oplus \mathfrak{p}_{h}^{-}\right) \subset$ $\mathfrak{U}\left(F^{0} \operatorname{Lie}\left(P^{\prime}\right)\right)$. We extend from $F^{0} \operatorname{Lie}\left(P^{\prime}\right)$ to $F^{0} \operatorname{Lie}(P)$ by letting $G_{\ell}$ act via the Levi decomposition above. Then as $\mathfrak{U}\left(F^{0} \operatorname{Lie}(P)\right)$-module the right-hand side of the last formula in $(4.2 .25)$ is

$$
\mathfrak{U}\left(F^{0} \operatorname{Lie}(P)\right) \otimes_{\mathfrak{U}(\mathfrak{r}(Q))} E(t)^{*}(s) .
$$

So substituting (4.2.25) into (4.2.24), we obtain

$H_{0}\left(\mathfrak{w}^{+}, \mathfrak{U}(\operatorname{Lie}(P)) \otimes \mathfrak{U}\left(F^{0} \operatorname{Lie}(P)\right) \wedge^{d_{P}}\left(\mathfrak{n}_{P}\right)^{*} \otimes H_{0}\left(\mathfrak{s}, \bigoplus_{\ell(s)=\bullet} \mathfrak{U}\left(F^{0} \operatorname{Lie}(P)\right) \otimes_{\mathfrak{U}(\mathfrak{r}(Q))} E(t)^{*}(s)\right)\right.$

which simplifies as

$$
H_{0}\left(\mathfrak{w}_{P}, \mathfrak{U}(\operatorname{Lie}(P)) \otimes_{\mathfrak{U}(\mathfrak{r}(Q))} E(t)^{*}(s)\right)=H_{0}\left(\mathfrak{w}_{P}, \mathbb{M}(\Lambda(t)(s))\right) .
$$

The same calculation, with $t$ replaced by $t^{\prime}$, shows that $R^{\bullet} \pi_{+} \mathcal{D}_{Z}\left(\mathcal{F}^{\vee}\right)$ is the automorphic vector bundle associated to

$$
\bigoplus_{\ell(s)=\bullet} H_{0}\left(\mathfrak{w}_{P}, \mathbb{M}\left(\Lambda\left(t^{\prime}\right)(s)\right)\right)
$$

We recognize (4.2.26) as the left-hand side of (4.2.14), where (unfortunately) $P$ and $\bar{P}$ have been interchanged. From here we apply Prop. 4.2 .15 (b) to obtain that the correspondence in Lemma 4.2.18 is compatible with the differentials on the two sides of (4.2.4), i.e. that for any $s \in W_{Q}^{\bar{P}}$ the linearization of the direct image

$$
R^{q} \pi_{*}^{(s)} \Delta\left(t, t^{\prime}\right): R^{q} \pi_{*}(\mathcal{E})^{s} \longrightarrow R^{q} \pi_{*}(\mathcal{F})^{s}
$$

is the homomorphism

$$
\mathcal{D}_{M}\left(R^{d_{P}-q} \pi_{*}(\mathcal{F})^{\vee}\right)^{s} \longrightarrow \mathcal{D}_{M}\left(R^{d_{P}-q} \pi_{*}\left(\mathcal{E}^{\vee}\right)\right)^{s}
$$

associated to the homomorphism $\mathbb{M}(\Lambda(t)(s)) \rightarrow \mathbb{M}\left(\Lambda\left(t^{\prime}\right)(s)\right)$ of generalized Verma modules. We conclude: 
THEOREM 4.2.27. - In the situation of (4.1.9), there is a canonical isomorphism of complexes:

$$
\begin{aligned}
& R\left(\bar{\pi}_{P}\right)_{*}\left(i_{R}^{*}\right)\{\mathcal{B G G}(M, \widetilde{\boldsymbol{E}})\}^{\mathrm{can}} \stackrel{\sim}{\longrightarrow} \\
& \bigoplus_{w \in W^{P}}\left\{\mathcal{B G G}\left(M_{P}, \widetilde{\boldsymbol{E}}_{\mu(h, w)}\right)^{\operatorname{can}} \otimes \boldsymbol{C}^{\bullet}\left(\Gamma_{\ell, R}, H^{\bullet}\left(E_{\mu(\ell, w)}\right)[-\ell(w)]\right\} .\right.
\end{aligned}
$$

Here the notation is as in (4.1.10.1).

We adelize the above theorem using the notation $\mathrm{Sh}_{\Sigma}, \mathrm{Sh}(R)$, etc., of (3.2). The least awkward expression is in terms of the inverse limit over all toroidal compactifications

$$
\widetilde{\mathrm{Sh}}=\widetilde{\mathrm{Sh}}(G, X)=\lim _{K_{f}, \Sigma} \mathrm{Sh}_{\Sigma}
$$

(recall that the level subgroup $K_{f}$ is implicit in the notation). Let $i_{R}: \widetilde{\mathrm{Sh}}^{R(*)} \hookrightarrow \widetilde{\mathrm{Sh}}$ denote the inclusion of the $R(*)$-stratum (inverse limit over all $R(*)$-strata at finite level). Let $\Delta_{1, R, h}$ denote the projection of the group $\Delta_{1, R}$ of (3.2) on $G_{h, P}\left(\boldsymbol{A}_{f}\right)$, and let $\operatorname{Sh}(R)^{+}$denote the quotient $\operatorname{Sh}\left(G_{h, P}, X(P)\right) / \Delta_{1, R, h}$. Then $R\left(\boldsymbol{A}_{f}\right)$ acts on $\operatorname{Sh}(R)^{+}$, and the adelic version of $\bar{\pi}_{P}$ is a natural morphism

$$
\bar{\pi}_{R}: \widetilde{\operatorname{Sh}}^{R(*)} \longrightarrow \operatorname{Ind}_{R\left(\boldsymbol{A}_{f}\right)}^{G\left(\boldsymbol{A}_{f}\right)} \widetilde{\operatorname{Sh}}(R)^{+},
$$

where $\widetilde{\mathrm{Sh}}(R)^{+}$is again the projective limit over all toroidal compactifications. For $w \in W^{R}$, let

$$
\begin{aligned}
\mathcal{B G G}(\operatorname{Sh}(R), w, E) & \\
& =\mathcal{B G G}\left(\operatorname{Sh}\left(G_{h, P}, X(P)\right), \widetilde{\boldsymbol{E}}_{\mu(h, w)}\right)^{\operatorname{can}} \otimes C^{\bullet}\left(X\left(G_{\ell, R}\right), E_{\mu(\ell, w)}\right)[-\ell(w)] .
\end{aligned}
$$

Then the formula (3.2.8), with the space $\mathcal{H}^{\bullet}(w)$ replaced by the complex $\mathcal{B G G}(\operatorname{Sh}(R), w, E)$, defines an $R\left(\boldsymbol{A}_{f}\right)$-equivariant vector bundle $I^{R}(\mathcal{B G G}(\operatorname{Sh}(R), w, E))$ on $\widetilde{\operatorname{Sh}}(R)^{+}$. The adelic version of Theorem (4.2.27) is

\section{THEOREM 4.2.30}

(a) In the above situation, there is a canonical isomorphism of complexes:

$$
R\left(\bar{\pi}_{P}\right)_{*}\left(i_{R}^{*}\right)\{\mathcal{B G G}(\widetilde{\operatorname{Sh}}(G, X), \widetilde{\boldsymbol{E}})\}^{\operatorname{can}} \stackrel{\sim}{\longrightarrow} \operatorname{Ind}_{R\left(\boldsymbol{A}_{f}\right)}^{G\left(\boldsymbol{A}_{f}\right)} \underset{w \in W^{R}}{\bigoplus}\left\{I^{R}(\mathcal{B G G}(\operatorname{Sh}(R)), w, E)\right) .
$$

(b) This isomorphism is rational over the reflex field $E(G, X)$.

Proof. - Part (a) is just the adelic version of (4.2.27), or rather of its obvious variant for the $R$-stratum. (Here we are using the fact that the Kostant decomposition for the parabolic subgroup $R_{\ell, P}$ of $G_{\ell, P}$ defines a direct sum decomposition in the derived category for the cohomology complex of the $R_{\ell, P}$-stratum of the boundary of $X\left(G_{\ell, P}\right)$.) Then (b) is immediate because the identification in Lemma 4.2.18 is rational over $E(G, X)$, as are all subsequent constructions. 
REMARK 4.2.31. - Upon taking fixed vectors for a level subgroup $K_{f}$, the above theorem can be given a reasonable expression in terms of toroidal compactifications at finite level.

\subsection{Hodge theory at the boundary, revisited}

Let $M_{P}^{\prime}$ denote a connected component of the mixed Shimura variety associated to a maximal parabolic subgroup $P$ of $G$, and

$$
M_{P}^{\prime} \stackrel{\pi_{2}}{\longrightarrow} A_{P} \stackrel{\pi_{1}}{\longrightarrow} M_{P}
$$

the associated tower of algebraic fibrations (see (1.4.1) or [HZ1,(1.2.5)]). Let, as usual, $\pi$ (or $\pi_{P}$ ) denote $\pi_{1} \circ \pi_{2}$. For $\rho: P \rightarrow G L(E)$ a rational representation, there are isomorphisms of local systems on $M_{P}$ :

$$
R^{k} \pi_{*} \widetilde{\boldsymbol{E}} \simeq \widetilde{\boldsymbol{H}}^{k}\left(\mathfrak{w}_{P}, E\right)
$$

This follows from the fact that the fibers of $\pi$ have the homotopy type of the compact nilmanifold $\Gamma_{W_{P}} \backslash W_{P}(\mathbb{R})$. In fact, (4.3.2) is a consequence of the more basic fact at the cochain level:

$$
R \pi_{*} \widetilde{\boldsymbol{E}} \simeq \pi_{*}\left\{\left.\widetilde{\boldsymbol{C}}^{\bullet}\left(\mathfrak{w}_{P}, E\right)\right|_{S_{L}}\right\}
$$

where $S_{L}$ is the cross-section of $\pi$ given by a choice of rational Levi subgroup $L \subset{ }^{0} P$ and basepoint $c_{P}\left(x_{0}\right)$ (the latter is fixed by $G_{\ell, P}$ ).

In $[\mathrm{HZ} 2, \S 5]$, we saw (among other things) that the above assertions remain true in the Hodge theoretic sense. As J. Wildeshaus has pointed out to us, the arguments in [HZ2] are really assertions about the associated mixed Hodge modules - much of it is already there - and we wish to recast them here.

Let $M_{P, \Sigma}^{\prime}$ denote the toroidal partial compactification of $M_{P}^{\prime}$ determined by $\Sigma$; it is not an algebraic variety, as its boundary has infinitely many irreducible components. It contains the boundary stratum that is called $<\widetilde{Z}_{P, \Sigma}$ in [HZ1]. Taking the quotient of the latter by $\Gamma_{\ell, P}$ gives rise to the $P$-stratum $<Z_{P, \Sigma}$ in $M_{\Sigma}$. And $Z_{\Sigma}(P)$ is the just the closure of $<Z_{P, \Sigma}$ in $M_{\Sigma}$. Of course, $Z_{\Sigma}(P)$ contains $Z_{\Sigma}(R)$ whenever $R \subseteq P$, but $Z_{\Sigma}(R)$ maps surjectively to $\left(M_{P}\right)_{\Xi}$ under the projection $\bar{\pi}: Z_{\Sigma}(P) \rightarrow\left(M_{P}\right)_{\Xi}$ (as in (4.1.10.2)) if and only if $R$ is subordinate to $P$.

We recall some constructions from $[\mathrm{HZ} 2,(5.2)]$. We denote by $j_{\Sigma}$ the inclusion of $M_{P}^{\prime}$ in $M_{P, \Sigma}^{\prime}$. Let $\mathcal{M}_{\Sigma}$ (called $\mathcal{M}$ in [HZ2]) be the unique mixed Hodge module on $M_{P, \Sigma}^{\prime}$ with underlying perverse sheaf $R j_{*} \widetilde{\boldsymbol{E}}$, such that its restriction to $M_{P}^{\prime}$ is the variation of mixed Hodge structure defined by the representation of $P$ on $E$. Then $\left(\pi_{*} \mathcal{M}_{\Sigma}, R \pi_{*} \widetilde{\boldsymbol{E}}\right)$ is a mixed Hodge module on $M_{P}$ with action of $\Gamma_{\ell, P}$.

Proposition 4.3.4 ([HZ2, (5.2.9),(5.4.19)]). — In the derived category of mixed Hodge modules, $\left(\pi_{*} \mathcal{M}_{\Sigma}, R \pi_{*} \widetilde{\boldsymbol{E}}\right)$ is $\Gamma_{\ell, P}$-equivariantly isomorphic to the complex of 
variations of mixed Hodge structure $\pi_{*}\left\{\left.\widetilde{\boldsymbol{C}}^{\bullet}\left(\mathfrak{w}_{P}, E\right)\right|_{S_{L}}\right\}$ with filtrations induced by the mixed Shimura data for $P$. In particular, the latter is admissible.

Though unusual in general, we expected in this case that the mixed sheaf $R \pi_{*} \widetilde{\boldsymbol{E}}$ would split as the direct sum of pure sheaves (of different weights). Indeed, the following is an immediate consequence of (4.3.4):

THEOREM 4.3.5. - In the derived category of mixed Hodge modules on $M_{P}$,

$$
R \pi_{*} \widetilde{\boldsymbol{E}} \approx \widetilde{\boldsymbol{H}}^{\bullet}(\mathfrak{w}, E) \simeq \bigoplus_{w \in W^{P}} \widetilde{\boldsymbol{E}}_{\mu(h, w)} \otimes E_{\mu(\ell, w)}[-l(w)]
$$

underlies a decomposition of mixed Hodge modules. Each $\widetilde{\boldsymbol{E}}_{\mu(h, w)}$, being determined by an irreducible representation of $G_{h, P}$, is pure.

Corollary 4.3.6 ([HZ2, (5.4.20)]). - The mixed Hodge structure of $H^{i}\left(M_{P}^{\prime}, \widetilde{\boldsymbol{E}}\right)$ decomposes as the direct sum of mixed Hodge structures:

$$
\bigoplus_{w \in W^{P}} H^{i-l(w)}\left(M_{P}, \widetilde{\boldsymbol{E}}_{\mu(h, w)}\right) \otimes E_{\mu(\ell, w)} .
$$

It is time to move on to the boundary cohomology of $Z_{\Sigma}=\partial M_{\Sigma}$. The final result in $[\mathrm{HZ} 2, \S 5]$ is the following, which should be compared to (4.3.5) and (4.3.6). It is the Hodge theoretic version of (4.1.9), and, by iteration, it provides the Hodge theoretic version of (4.1.19).

THEOREM 4.3.7 ([HZ2,(5.6.10)]). - Let $E$ be a representation of $G$, so that $\widetilde{\boldsymbol{E}}$ underlies a variation of Hodge structure on $M$. Let $j_{P}: M_{P} \rightarrow M_{P, \Sigma(P)}$ be a suitable toroidal compactification of $M_{P}$, as in (3.1.2). Suppose that $\Pi(R)=P$. Then

(i) there is a decomposition in the derived category of mixed Hodge modules on $M_{P, \Sigma(P)}$

$$
\begin{aligned}
R \bar{\pi}_{*} i_{R}^{*} \widetilde{\boldsymbol{E}} & \approx \bigoplus_{w \in W^{P}} R\left(j_{P}\right)_{*} \widetilde{\boldsymbol{E}}_{\mu(h, w)} \otimes C^{\bullet}\left(\Gamma_{\ell, R}, E_{\mu(\ell, w)}\right)[-l(w)] \\
& \approx \bigoplus_{w \in W^{R}} R\left(j_{P}\right)_{*} \widetilde{\boldsymbol{E}}_{\mu(h, w)} \otimes C^{\bullet}\left(\Gamma_{\ell, R}^{\mathrm{red}}, E_{\mu(\ell, w)}\right)[-l(w)] \\
& \approx R\left(j_{P}\right)_{*} C^{\bullet}\left(\Gamma_{\ell, R}, \widetilde{\boldsymbol{H}}^{\bullet}(\mathfrak{w}, E)\right) ;
\end{aligned}
$$

(ii) there is a decomposition of mixed Hodge structures

$$
H_{d n}^{q}\left(Z_{\Sigma}(R), \widetilde{\boldsymbol{E}}\right) \simeq \bigoplus_{a ; w \in W^{P}} H^{a}\left(M_{P}, \widetilde{\boldsymbol{E}}_{\mu(h, w)}\right) \otimes H^{q-a-l(w)}\left(\Gamma_{\ell, R}, E_{\mu(\ell, w)}\right) .
$$

REMARK. - The passage from the sum over $W^{P}$ to the sum over $W^{R}$ is an isomorphism of mixed Hodge modules; cf. Remark (3.1.9)(ii)(b).

There are four main ingredients in the proof of the above assertions: the simplicial structure of the boundary (which produces the $\Gamma_{\ell, R^{-}}$cohomology in (ii) above), the calculation on $M_{P}^{\prime}$ for a single simplex, the irrelevance of the boundary of $M_{P}$, and 
the inability of the boundary cohomology here to distinguish the variation of mixed Hodge structure from $M_{P}^{\prime}$ from the variation of pure Hodge structure on $M$.

For the second item, one reverts back to $M_{P, \Sigma}^{\prime}$, as the structure of both at the $P$-stratum is locally the same. Let $\tau$ be any cone in $\Sigma_{P}$, and $i_{\tau}: Z_{\tau} \rightarrow M_{P, \Sigma}^{\prime}$ the inclusion. Then,

Proposition 4.3.8. - There is an isomorphism in the derived category of mixed Hodge modules on $M_{P}$, given by restriction of that in (4.3.4):

$$
R \pi_{*} i_{\tau}^{*} R j_{*} \widetilde{\boldsymbol{E}} \approx \pi_{*}\left\{\left.\widetilde{\boldsymbol{C}}^{\bullet}\left(\mathfrak{w}_{P}, E\right)\right|_{S_{L}}\right\} .
$$

The next proposition, tacit in $[\mathrm{HZ} 2, \S 5]$, asserts that a divisor with normal crossings behaves like the boundary of a manifold-with-corners with regard to deleted neighborhood cohomology. (This is consistent with the comparison of nerves in [HZ2, (2.7.8)].)

Proposition 4.3.9. - Let $D$ be a divisor with normal crossings, whose irreducible components will be denoted $D_{i}$, on the complex manifold $Y$, and let $j: Y-D \hookrightarrow Y$ be the inclusion. Let $D_{A}=\bigcap_{i \in A} D_{i}$ be an intersection of components of $D$, and denote by $D_{A}{ }^{\circ}$ the set of points of $D_{A}$ that lie on no additional components of $D$, and $\nu_{A}: D_{A}^{\circ} \hookrightarrow D_{A}$. Finally, let $i_{A}$ and $i_{A}^{\circ}$ denote the respective inclusions of $D_{A}$ and $D_{A}^{\circ}$ in $Y$. Then for any local system $\widetilde{\boldsymbol{L}}$ on $Y-D$,

$$
i_{A}^{*} R j_{*} \widetilde{\boldsymbol{L}} \stackrel{\sim}{\longrightarrow} R \nu_{A, *}\left(i_{A}^{\circ}\right)^{*} R j_{*} \widetilde{\boldsymbol{L}}
$$

Proof. - The assertion is local on $D_{A}$. Also, there is nothing to prove at points of $D_{A}{ }^{\circ}$. Thus, suppose that $y \notin D_{A}{ }^{\circ}$, and let $D_{B}$ denote the (non-empty) intersection of the additional divisors passing through $y$. Then there are local coordinates centered at $y$ such that near $y, j: Y-D \hookrightarrow Y$ is $\left(\Delta^{*}\right)^{a} \times\left(\Delta^{*}\right)^{b} \times \Delta^{n-b-a} \subset \Delta^{n}$, where $a=\# A$ and $b=\# B$. In these terms, $D_{A}{ }^{\circ}=\{0\} \times\left(\Delta^{*}\right)^{b} \times \Delta^{n-b-a}$, and $D_{A}=\{0\} \times \Delta^{b} \times$ $\Delta^{n-b-a}$. Thus, we may as well assume that $n=b+a$. Then the stalk of $i_{A}{ }^{*} R j_{*} \widetilde{\boldsymbol{L}}$ is $C^{\bullet}\left(\left(\Delta^{*}\right)^{a} \times\left(\Delta^{*}\right)^{b}, \widetilde{\boldsymbol{L}}\right)$, while that of $R \nu_{A, *}\left(i_{A}^{\circ}\right)^{*} R j_{*} \widetilde{\boldsymbol{L}}$ is $C^{\bullet}\left(\left(\Delta^{*}\right)^{b}, C^{\bullet}\left(\Delta^{*}\right)^{a}, \widetilde{\boldsymbol{L}}\right)$. In both cases, one has a complex for computing $H^{\bullet}\left(\left(\Delta^{*}\right)^{a} \times\left(\Delta^{*}\right)^{b}, \widetilde{\boldsymbol{L}}\right)$, and our assertion follows.

In short, one does not have to worry about the compactification of a stratum of the boundary in treating the deleted neighborhood cohomology. This addresses the third item in the outline of the proof of (4.3.7) above.

The fourth and final one is worded imprecisely, and we elaborate now. As mentioned before, $\Gamma_{\ell, P} \backslash<\widetilde{Z}_{P, \Sigma}$ is analytically isomorphic to $<Z_{P, \Sigma}$; indeed, there is a $\Gamma_{\ell, P}$-invariant deleted neighborhood $\widetilde{O}$ of $<\widetilde{Z}_{P, \Sigma}$ in $M_{P, \Sigma}^{\prime}$ such that $\Gamma_{\ell, P} \backslash \widetilde{O}$ is analytically equivalent to an deleted neighborhood $O$ of $<Z_{P, \Sigma}$ in $M_{\Sigma}$.

Of course, the local system $\left.\widetilde{\boldsymbol{E}}\right|_{O}$ underlies a variation of (pure) Hodge structure, viz., the restriction of the one on $M$. On the other hand, the variation of mixed Hodge structure determined by $E$ as a representation of $P$ is $\Gamma_{\ell, P}$-equivariant, so its 
restriction to $\widetilde{O}$ descends to a variation of mixed Hodge structure on $O$, with the same underlying local system; it is an admissible variation. To distinguish them as mixed Hodge modules, we write $\left.\widetilde{\boldsymbol{E}}_{(G)}\right|_{O}$ and $\widetilde{\boldsymbol{E}}_{(P)} \mid O$ resp. The following is an elaboration on $[\mathrm{HZ2},(5.6 .12)]$ :

Proposition 4.3.10. - Suppose that $\Pi(R)=P$. Then the mixed Hodge modules $i_{R}^{*} \widetilde{\boldsymbol{E}}_{(G)} \mid O$ and $i_{R}^{*} \widetilde{\boldsymbol{E}}_{(P)} \mid O$ are isomorphic.

Proof. - We begin by recalling that $\widetilde{\boldsymbol{E}}_{(G)} \mid O$ and $\widetilde{\boldsymbol{E}}_{(P)} \mid O$ have the same Hodge filtration, but they differ in their weight filtrations. The latter are determined respectively by the weight homomorphisms $w_{G}$ and $w_{P}$ (as in [HZ1, 1.2.2], where the first one is written $h \circ w)$. As such, they have the same asymptotic Hodge filtrations, and we want to show that the associated weight filtrations along $Z_{\Sigma}(R)$ coincide. Let $Z_{\Sigma}^{\circ}(R)$ be the locus of smooth points of $Z_{\Sigma}(R)$. By (4.3.9) and the uniqueness property $[\mathrm{Sa} 3,2.11]$, it is enough to verify that the weights coincide along $Z_{\Sigma}^{\circ}(R)$.

The local monodromy transformations of $\widetilde{\boldsymbol{E}}$ are unipotent. Until we say otherwise, we continue in the setting of an arbitrary admissible variation of mixed Hodge structure $(\widetilde{\boldsymbol{V}}, \widetilde{\boldsymbol{W}}, \mathcal{F})$ with unipotent local monodromy, defined in a neighborhood of $Z_{\Sigma}^{\circ}(R)$. Then $i_{R}^{*}(\widetilde{\boldsymbol{V}}, \widetilde{\boldsymbol{W}}, \mathcal{F})$, can be determined as a mixed Hodge module by iteration on the following construction (iterated deleted neighborhood cohomology, discussed in [HZ2, (3.1.7)]). Let $y \in Z_{\Sigma}^{\circ}(R)$; because $\Pi(R)=P$, there is a component $D$ of $Z_{\Sigma}(P)$ passing through $y$. Also, let $i_{D} \hookrightarrow M_{\Sigma}$ be the inclusion. Then $i_{D}^{*}(\widetilde{\boldsymbol{V}}, \widetilde{\boldsymbol{W}}, \mathcal{F})$ is a one-variable degeneration with $D$ as "parameter". ${ }^{(2)}$

The issues that occur are at the level of a filtered local system on the punctured disc $\Delta^{*}$ (the situation transverse to $D$ ) with unipotent monodromy. Let $N$ denote the (nilpotent) monodromy logarithm, acting on any reference fiber $V$ of $\widetilde{\boldsymbol{V}}$. The weight filtration of $i_{D}^{*}(\widetilde{\boldsymbol{V}}, \widetilde{\boldsymbol{W}}, \mathcal{F})$ is given by the weight filtration of $N$ relative to $W$, which is denoted $M(N ; W)$. If $W$ is trivial, then $M(N ; W)$ is the absolute weight filtration $M(N)$ of $N$, equal to the convolution of the kernel and image filtrations of $N$ (see $[\mathrm{StZ},(2.3)])$, which is characterized by the statement

$$
\begin{aligned}
& N M_{k}(N) \subseteq M_{k-2}(N) \text {, and } \\
& \qquad N^{k}: \mathrm{Gr}_{k}^{M(N)} V \longrightarrow \mathrm{Gr}_{-k}^{M(N)} V \text { is an isomorphism. }
\end{aligned}
$$

In general, $M(N ; W)$ is characterized (if it exists) by

$$
\begin{aligned}
& N M_{k}(N ; W) \subseteq M_{k-2}(N ; W), \text { and } \\
& \qquad N^{k}: \operatorname{Gr}_{k+i}^{M(N ; W)} \operatorname{Gr}_{i}^{W} V \longrightarrow \mathrm{Gr}_{-k+i}^{M(N ; W)} \operatorname{Gr}_{i}^{W} V \text { is an isomorphism; }
\end{aligned}
$$

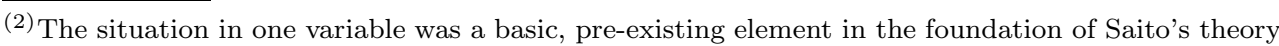
of mixed Hodge modules. 
in other words, $M(N ; W)$ induces $M\left(\mathrm{Gr}_{i}^{W} N\right)[i]$ on $\mathrm{Gr}_{i}^{W} V$. In total generality, there is no reason that a filtration satisfying (4.3.12) should exist. However, it is one of the conditions defining admissibility that the relative weight filtration exist.

At this point, one appeals to the following fact that we used in [HZ2, (5.6)]:

Proposition 4.3.13 ([StZ, (2.14)]). - Let $V$ be a vector space with increasing filtration $W$, and $N$ a nilpotent endomorphism of $V$ such that $N W_{i} \subseteq W_{i-1}$. Then $M(N ; W)$ exists if and only if $N W_{i} \subseteq W_{i-2}$, in which case $M(N ; W)=W$.

We check that this is satisfied in the Shimura variety setting. Let $\sigma$ be the onedimensional cone in $\Sigma_{P}^{c}$ that defines $D$. The monodromy $\operatorname{logarithm} N$ around $D$ is given by some non-zero integral element of $\sigma$, and this is interior to $C_{P}$. Thus, its weight filtration is just the one defined by $w_{P}$. This lowers weights by two, so by (4.3.13), $M(N ; W)=W$, which is also $M(N)$. Thus, there is an isomorphism of mixed Hodge modules:

$$
\left.\left.i_{D}^{*} \widetilde{\boldsymbol{E}}_{(P)}\right|_{O} \simeq i_{D}^{*} \widetilde{\boldsymbol{E}}_{(G)}\right|_{O} .
$$

Using the fact that $i_{R}^{\circ}=i_{D} \circ\left(i_{R}^{D}\right)^{\circ}$, where $\left(i_{R}^{D}\right)^{\circ}$ denotes the inclusion of $Z_{\Sigma}^{\circ}(R)$ in $D$, we get that

$$
i_{R}^{*} \widetilde{\boldsymbol{E}}_{(P)}\left|O \simeq i_{R}^{*} \widetilde{\boldsymbol{E}}_{(G)}\right| O,
$$

on $Z_{\Sigma}^{\circ}(R)$, hence on $Z_{\Sigma}(R)$, which is what we needed to show.

We conclude by formulating the nerve spectral sequence in the language of this section:

ThEOREM 4.3.14 (see [HZ2, (5.5.2)])

(i) The mixed Hodge module $i^{*} R j_{*} \widetilde{\boldsymbol{E}}$ is canonically isomorphic in the derived category of mixed Hodge modules to the chain complex $S_{\bullet}$, where

$$
S_{r}=\bigoplus_{r(R)=r+1} i_{R}^{*} R j_{*} \widetilde{\boldsymbol{E}}
$$

and the differentials are given by restriction. On hypercohomology, the spectral sequence associated to filtration by degree in the latter is the nerve spectral sequence, which is thereby a spectral sequence of mixed Hodge structures.

(ii) Whenever $\Pi(R)=P$, one has, as in (4.3.7)(i),

$$
\begin{aligned}
R\left(\bar{\pi}_{P}\right)_{*} i_{R}^{*} R j_{*} \widetilde{\boldsymbol{E}} & \approx R\left(j_{P}\right)_{*} C^{\bullet}\left(\Gamma_{\ell, R}, \widetilde{\boldsymbol{H}}^{\bullet}\left(\mathfrak{w}_{P}, E\right)\right) \\
& \approx \bigoplus_{w \in W^{P}} R\left(j_{P}\right)_{*} \widetilde{\boldsymbol{E}}_{\mu(h, w)} \otimes C^{\bullet}\left(\Gamma_{\ell, R}, E_{\mu(\ell, w)}\right)[-l(w)] .
\end{aligned}
$$


(iii) Grading for the Hodge filtration $F$, one has that $\operatorname{Gr}_{F}^{p}\left(i^{*} R j_{*} \widetilde{\boldsymbol{E}}\right)$ is canonically quasi-isomorphic to $\operatorname{Gr}_{F}^{p} S_{\bullet}$. The corresponding "nerve" spectral sequence is:

$$
\begin{aligned}
E_{1}^{r, s} & =\bigoplus_{r(R)=r+1} H^{s}\left(Z_{R}, i_{R}^{*} \operatorname{Gr}_{F}^{p}\left(R j_{*} \widetilde{\boldsymbol{E}}\right)\right) \\
& \simeq \bigoplus_{r(R)=r+1} H^{s}\left(Z_{R}, i_{R}^{*} \operatorname{Gr}_{F}^{p} \mathcal{B B} \mathcal{G}(M, \widetilde{\boldsymbol{E}})^{\mathrm{can}}\right) \Longrightarrow H^{r+s}\left(Z, i^{*} \operatorname{Gr}_{F}^{p} \mathcal{B B} \mathcal{G}(M, \widetilde{\boldsymbol{E}})^{\mathrm{can}}\right) .
\end{aligned}
$$

(iv) Whenever $\Pi(R)=P$, one has:

$$
R\left(\bar{\pi}_{P}\right)_{*} i_{R}^{*} \operatorname{Gr}_{F}^{p} \mathcal{B B G}(M, \widetilde{\boldsymbol{E}})^{\text {can }} \approx C^{\bullet}\left(\Gamma_{\ell, R},\left\{\mathcal{H}^{\bullet}\left(\mathfrak{s}_{p, R}, \operatorname{Gr}_{F}^{p} B B G(M, \widetilde{\boldsymbol{E}})\right)\right\}^{\text {can }}\right) .
$$

\section{REMARKS 4.3.15}

(i) Theorem (4.3.14) has an adelic version, along the lines of Theorem (4.2.29), whose formulation we leave to the reader, noting that it is most easily effected upon replacing the sum over $W^{P}$ by a sum over $W^{R}$; see the remark following (4.3.7) and the horizontal arrows in (ii), below. We also note that (4.2.29)(b) implies that (4.3.14.)(i) defines a canonical isomorphism in the derived category of mixed Hodge modules with $E(G, X)$-rational structure.

(ii) Proposition (3.4.1) also has a version in terms of mixed Hodge modules: if $R \supset R^{\prime}$ are two proper parabolics of $G$, with $r\left(R^{\prime}\right)=r(R)+1$, then the following diagram commutes:

$$
\begin{aligned}
& R\left(\bar{\pi}_{R}\right)_{*} i_{R}^{*} R j_{*}(\widetilde{\boldsymbol{E}}) \stackrel{\approx}{\longrightarrow} \operatorname{Ind}_{R\left(\boldsymbol{A}_{f}\right)}^{G\left(\boldsymbol{A}_{f}\right)} \bigoplus_{w \in W^{R}}\left\{I^{R}\left(R\left(j_{R}\right)_{*} C^{\bullet}\left(X\left(G_{\ell, R}\right), E_{\mu(\ell, w)}\right)[-\ell(w)]\right)\right\} \\
& R\left(\bar{\pi}_{R^{\prime}}\right)_{*} i_{R^{\prime}}^{*} R j_{*}(\widetilde{\boldsymbol{E}}) \stackrel{\downarrow}{\underset{\sim}{\longrightarrow} \operatorname{Ind}_{R^{\prime}\left(\boldsymbol{A}_{f}\right)}^{G\left(\boldsymbol{A}_{f}\right)}} \underset{w \in W^{R^{\prime}}}{\bigoplus}\left\{I^{R^{\prime}}\left(R\left(j_{R^{\prime}}\right)_{*} \stackrel{\downarrow}{ } C^{\bullet}\left(X\left(G_{\ell, R^{\prime}}\right), E_{\mu(\ell, w)}\right)[-\ell(w)]\right)\right\}
\end{aligned}
$$

Here the horizontal arrows are the quasi-isomorphisms of (4.3.14.)(ii), and the lefthand vertical arrow is defined by the natural restriction. When $\Pi(R)=\Pi\left(R^{\prime}\right)$ (resp. $\left.\Pi(R) \neq \Pi\left(R^{\prime}\right)\right)$ the right-hand vertical arrow is determined as in (3.2.9)(ii) (resp. as in (3.4.1)). We leave the precise formulation to the reader, noting only that in the $\mathcal{B G G}$ realization, the quasi-isomorphisms can be replaced by isomorphisms of complexes.

\subsection{The topological cohomology}

As usual, let $R \in \mathcal{P}(G)$. In the "standard" way of doing things, one writes $R=$ $L_{R} \cdot W_{R}$; then by $[\mathrm{Ha}]$,

$$
H^{\bullet}\left(\overline{e^{\prime}(R)}, \widetilde{\boldsymbol{E}}\right) \simeq H^{\bullet}\left(e^{\prime}(R), \widetilde{\boldsymbol{E}}\right) \simeq H^{\bullet}\left(\Gamma_{R}, E\right) \simeq H^{\bullet}\left(X\left(\Gamma_{L_{R}}\right), \widetilde{\boldsymbol{H}}^{\bullet}\left(\mathfrak{w}_{R}, E\right)\right) .
$$

In actuality, the above holds at the cochain level, which can be viewed as an instance of (2.7.8):

$$
C^{\bullet}\left(\overline{e^{\prime}(R)}, \widetilde{\boldsymbol{E}}\right) \approx C^{\bullet}\left(X\left(\Gamma_{L_{R}}\right), \widetilde{\boldsymbol{H}} \cdot\left(\mathfrak{w}_{R}, E\right)\right) \approx C^{\bullet}\left(\Gamma_{L_{R}}, H^{\bullet}\left(\mathfrak{w}_{R}, E\right)\right)
$$


If we have that $\Pi(R)=P$, then $G_{h, P}$ is a direct factor of $L_{R}$; indeed, we have, as we noted before, that $R=G_{h, P} \cdot Q \cdot W_{P}$, for $Q$ a parabolic subgroup of $G_{\ell, P}$. The group $\Gamma \cap Q$ has been denoted $\Gamma_{\ell, R}$ (see (3.1.4)). When this is taken into account, we continue as in (3.3) to see that $(4.4 .2)$ can be written as:

$$
C^{\bullet}\left(e^{\prime}(R), \widetilde{\boldsymbol{E}}\right) \approx C^{\bullet}\left(\Gamma_{h, P} \cdot \Gamma_{\ell, R}, H^{\bullet}\left(\mathfrak{w}_{P}, E\right)\right) .
$$

We observe that the only thing on the right-hand side of (4.4.3) that varies when $R$ is, restricted to be subordinate to $P$, is the " $\Gamma_{\ell, R}$ ".

Analogous to (3.5.1), a complex for computing the topological cohomology of the Borel-Serre boundary $\partial \bar{X}$ is the double complex

$$
\bar{K}^{r, s}=\bigoplus_{r(R)=r+1} C^{s}\left(e^{\prime}(R), \widetilde{\boldsymbol{E}}\right),
$$

in which the differential $\widetilde{d}$ is the sum of the differential of $C^{\bullet}$ (which increases $s$ by one), and restriction (which increases $r$ by one). We denote the latter again by $d_{\text {par }}$. The nerve spectral sequence in this setting (see $[\mathrm{HZ2},(3.5)]$ ) is associated to the filtration $\mathcal{R}$ by rank, starting from the analogue of (3.5.2):

$$
\operatorname{Gr}_{\mathcal{R}}^{t} \bar{K}^{\bullet}=\bigoplus_{r(R)=t+1} C^{\bullet}\left(e^{\prime}(R), \widetilde{\boldsymbol{E}}\right)[-t]
$$

in which $d_{\text {par }}$ vanishes. This produces for the $E_{1}$-term, as in (4.1.15):

$$
\begin{aligned}
E_{1}^{p, q}\left(\bar{K}^{\bullet}, \mathcal{R}\right)=\bigoplus_{r(R)=p+1} E_{1}^{p, q}(R), \quad \text { where } \\
E_{1}^{p, q}(R)=H^{q}\left(e^{\prime}(R), \widetilde{\boldsymbol{E}}\right) \\
\simeq \bigoplus_{a+j+k=q} H^{a}\left(M_{P}, \widetilde{\boldsymbol{H}}^{k}\left(\Gamma_{\ell, R}, H^{j}\left(\mathfrak{w}_{P}, E\right)\right)\right) \quad \text { by (4.4.3). }
\end{aligned}
$$

When we invoke Kostant's theorem, this time for the rational parabolic subgroup $P$ of $G$, with Levi subgroup $G_{h, P} \times G_{\ell, P}$ :

$$
H^{\bullet}\left(\mathfrak{w}_{P}, E\right) \simeq \bigoplus_{w \in W^{P}}\left(E_{\mu(h, w)} \otimes E_{\mu(\ell, w)}\right)[-l(w)],
$$

we obtain

$$
\begin{aligned}
E_{1}^{p, q}(R) & \simeq \bigoplus_{a ; w \in W^{P}} H^{a}\left(M_{P}, \widetilde{\boldsymbol{E}}_{\mu(h, w)}\right) \otimes H^{q-a-l(w)}\left(\Gamma_{\ell, R}, E_{\mu(\ell, w)}\right) \\
& \simeq \bigoplus_{a ; w \in W^{P}} H^{a}\left(M_{P}, \widetilde{\boldsymbol{E}}_{\mu(h, w)}\right) \otimes H^{q-a-l(w)}\left(X\left(\Gamma_{\ell, R}\right), \widetilde{\boldsymbol{E}}_{\mu(\ell, w)}\right) .
\end{aligned}
$$

There is some reason to work instead with the filtration $\mathcal{R}^{h}$ by holomorphic rank, which is the filtration determined by the decomposition of $\bar{M}$ induced by the natural mapping of $\bar{X}$ onto the Baily-Borel compactification of $M$ (see [Z6,(1.6)(11)]). For 
this, we have instead

$$
\begin{aligned}
& \mathcal{R}_{t}^{h} \bar{K}^{\bullet}=\bigoplus_{\rho_{h}(R) \leqslant t} C^{\bullet}\left(e^{\prime}(R), \widetilde{\boldsymbol{E}}\right)[1-r(R)] \\
& \mathrm{Gr}_{t}^{\mathcal{R}^{h}} \bar{K}^{\bullet}=\bigoplus_{\rho_{h}(R)=t} C^{\bullet}\left(e^{\prime}(R), \widetilde{\boldsymbol{E}}\right)[1-r(R)] \\
& =\underset{P \sim P_{\rho-t}}{\bigoplus^{\bullet}} C^{\bullet}\left(\Gamma_{h, P}, K_{c}^{\bullet}\left(X\left(\Gamma_{\ell, P}\right), H^{\bullet}\left(\mathfrak{w}_{P}, E\right)\right)\right),
\end{aligned}
$$

with $K_{c}^{\bullet}$ as in (3.5.7), and the differential induced by $d+d^{\ell}$.

REMARK. - We note that the differential $d^{h}$, viewed as a summand of $d_{\text {par }}$, decomposes canonically as

$$
d^{h}=\sum_{r} d^{h, r}
$$

where, $d^{h, r}$ decreases holomorphic rank by $r$, and increases $\mathcal{R}^{\ell}$ by $r-1$.

From (4.4.9)(ii), we can now write down a convenient expression for the $E_{1}$-term of the holomorphic rank spectral sequence (cf. (3.5.8)):

Proposition 4.4.10. - In the spectral sequence for $\mathcal{R}^{h}$, the $E_{1}$-term is

$$
E_{1}^{p, q}\left(\bar{K}^{\bullet}, \mathcal{R}^{h}\right)=\underset{P \sim P_{\rho+p}}{\bigoplus_{1}} \widetilde{E}_{1}^{p, q}(P)
$$

where

$$
\begin{aligned}
\widetilde{E}_{1}^{p, q}(P) & =\bigoplus_{a+j+k=p+q} H^{a}\left(M_{P}, \widetilde{\boldsymbol{H}}_{c}^{k}\left(X\left(\Gamma_{\ell, P}\right), \widetilde{\boldsymbol{H}}^{j}\left(\mathfrak{w}_{P}, E\right)\right)\right) \\
& =\bigoplus_{a+j+k=p+q} H^{a}\left(\Gamma_{h, P}, H_{c}^{k}\left(X\left(\Gamma_{\ell, P}\right), \widetilde{\boldsymbol{H}}^{j}\left(\mathfrak{w}_{P}, E\right)\right)\right) \\
& =\bigoplus_{a+j+k=p+q} H_{c}^{k}\left(X\left(\Gamma_{\ell, P}\right), \widetilde{\boldsymbol{H}}^{a}\left(M_{P}, \widetilde{\boldsymbol{H}}^{j}\left(\mathfrak{w}_{P}, E\right)\right)\right) .
\end{aligned}
$$

NB-The non-trivial terms in the above are for $p \leqslant 0$.

Proof. - We use Kostant's theorem, again applied to $P$ (4.4.7). This enables us to use the Künneth formula to determine that

$$
\widetilde{E}_{1}^{p, q}(P)=\bigoplus_{a ; w \in W^{P}} H^{a}\left(M_{P}, \widetilde{\boldsymbol{E}}_{\mu(h, w)}\right) \otimes H_{c}^{p+q-a-l(w)}\left(X\left(\Gamma_{\ell, P}\right), \widetilde{\boldsymbol{E}}_{\mu(\ell, w)}\right) .
$$

We now recombine the terms:

$$
\begin{aligned}
\widetilde{E}_{1}^{p, q}(P) & =\bigoplus_{a ; w \in W^{P}} H^{a}\left(M_{P}, \widetilde{\boldsymbol{E}}_{\mu(h, w)} \otimes H_{c}^{p+q-a-l(w)}\left(X\left(\Gamma_{\ell, P}\right), \widetilde{\boldsymbol{E}}_{\mu(\ell, w)}\right)\right) \\
& =\bigoplus_{a ; w \in W^{P}} H^{a}\left(M_{P}, \widetilde{\boldsymbol{H}}_{c}^{p+q-a-l(w)}\left(X\left(\Gamma_{\ell, P}\right), \widetilde{\boldsymbol{E}}_{\mu(\ell, w)} \otimes \widetilde{\boldsymbol{E}}_{\mu(h, w)}\right)\right) \\
& =\bigoplus_{a+j+k=p+q} H^{a}\left(M_{P}, \widetilde{\boldsymbol{H}}_{c}^{k}\left(X\left(\Gamma_{\ell, P}\right), \widetilde{\boldsymbol{H}}^{j}\left(\mathfrak{w}_{P}, E\right)\right)\right),
\end{aligned}
$$


which is the first formula for $\widetilde{E}_{1}^{p, q}(P)$ above. The second formula is equivalent, and the third is proved similarly.

We wish to elaborate on the relation between the filtration by holomorphic rank and the Baily-Borel compactification $M^{*}$ of $M=M_{\Gamma}$ that was mentioned before (4.4.9). Let $f: \bar{M} \rightarrow M^{*}$ be the natural map. Our first observation is that, by degeneration of the relevant spectral sequences, one has the following and its corollary:

Proposition 4.4.11. - $\left.\left(R^{i} f_{!} \widetilde{\boldsymbol{E}}\right)\right|_{M_{P}} \simeq \bigoplus_{j+k=i} \widetilde{\boldsymbol{H}}_{c}^{k}\left(X\left(\Gamma_{\ell, P}\right), \widetilde{\boldsymbol{H}}^{j}\left(\mathfrak{w}_{P}, E\right)\right)$.

As we are using a mixture of compact and closed supports, we introduce the symbol $H_{\ell, c}$ to refer to that. Then

Corollary 4.4.12. - We have the formula for $\widetilde{E}_{1}^{p, q}(P)$ of (4.4.10):

$$
\widetilde{E}_{1}^{p, q}(P) \simeq \bigoplus_{a+i=p+q} H^{a}\left(M_{P}, R^{i} f_{!} \widetilde{\boldsymbol{E}}\right) \simeq H_{\ell, c}^{p+q}\left(e^{\prime}(P), \widetilde{\boldsymbol{E}}\right) .
$$

We consider $\partial M^{*}$ as a filtered space. Specifically, one has

$$
\partial M^{*}=\underset{P \text { maximal }}{\bigsqcup} M_{P}
$$

(as a set), and we put for each integer $t$

$$
Y_{t}=\underset{\operatorname{hol} \mathrm{rk} P \leqslant t}{\bigsqcup} M_{P} .
$$

This defines a finite increasing filtration of $\partial M^{*}$, which we also call $Y_{\infty}$, by closed subspaces. Note that $R f_{!} \widetilde{\boldsymbol{E}}$ is constructible with respect to the stratification of $M^{*}$ induced from (4.4.13), and we have for all $P$ :

$$
\mathbb{H} \bullet\left(M_{P}, R f_{!} \widetilde{\boldsymbol{E}}\right) \simeq H_{\ell, c}^{\bullet}\left(e^{\prime}(P), \widetilde{\boldsymbol{E}}\right) .
$$

We now assert:

Proposition 4.4.15. - The spectral sequence for the filtration by holomorphic rank (see (4.4.10)) coincides with that of the filtered space $\partial M^{*}$, with filtration given in (4.4.13), and with coefficients in $\mathcal{F}=R f_{!} \widetilde{\boldsymbol{E}}$ :

$$
E_{1}^{p, q}=H^{p+q}\left(Y_{-p}, Y_{-p-1} ; \mathcal{F}\right) \Longrightarrow H^{p+q}\left(Y_{\infty} ; \mathcal{F}\right) .
$$

Proof. - Let $\bar{Y}_{t}=f^{-1} Y_{t}$, a closed subset of $\partial \bar{M}$ that consists of a union of maximal faces. As such, it is topologically a manifold-with-boundary. Note that the boundary of a maximal face $\overline{e^{\prime}(P)}$ consists of faces $\overline{e^{\prime}(R)}$ with $R \subset P$; then $f\left(e^{\prime}(R)\right) \subset M_{P}$ if and only if $R$ is subordinate to $P$. It follows that $\bar{Y}_{t}$ is the union of those $\overline{e^{\prime}(R)}$ for which $\rho_{h}(R) \leqslant t$. From (4.4.9)(i), one sees that the cochain complex $\mathcal{R}_{t}^{h} \bar{K}^{\bullet}$ is quasi-isomorphic to

$$
C \cdot\left(\bar{Y}_{t}, \partial \bar{Y}_{t} ; \widetilde{\boldsymbol{E}}\right) \approx C_{c}^{\bullet}\left(\bar{Y}_{t}-\partial \bar{Y}_{t} ; \widetilde{\boldsymbol{E}}\right) \approx C \cdot\left(Y_{t} ; R f_{!} \widetilde{\boldsymbol{E}}\right) .
$$

This proves our assertion. 
The following elementary fact, which is essentially (3.3.4), is fundamental in the determination of the differential $d_{1}$ in the holomorphic rank spectral sequence:

Lemma 4.4.16. - Let $R$ be a parabolic subordinate to $P$, and let

$$
L_{R}=G_{\ell, R} \cdot G_{h, P}
$$

be the associated decomposition of its Levi subgroup. Let $P^{\prime}$ be a maximal parabolic with $P^{\prime} \prec P$, and put $R^{\prime}=R \cap P^{\prime}$. Then the decomposition of $L_{R^{\prime}}$ is

$$
L_{R^{\prime}}=G_{\ell, R^{\prime}} \cdot G_{h, P^{\prime}}, \quad \text { with } \quad G_{\ell, R^{\prime}}=G_{\ell, R} \cdot\left(G_{\ell, P^{\prime}} \cap G_{h, P}\right) \text {. }
$$

In particular, when $P^{\prime}$ and $P$ are consecutive, $G_{\ell, R^{\prime}}=G_{\ell, R}$.

When $P^{\prime}$ and $P$ are consecutive, we thus have that

$$
R^{\prime}=L_{R^{\prime}} \cdot W_{R^{\prime}}=G_{\ell, R} \cdot G_{h, P^{\prime}} \cdot\left(W_{R} \cdot W_{P^{\prime}}\right)
$$

is contained in $R=G_{\ell, R} \cdot G_{h, P} \cdot W_{R}$. If $\Gamma$ is sufficiently small, one has the corresponding decomposition of $\Gamma_{R^{\prime}}$. One can elect to factor out $W_{P}$ instead; it is just a question of whether one is viewing $R^{\prime}$ as a parabolic subgroup of $P$ or of $P^{\prime}$.

At bottom, $d^{h}$ is given by $R \mapsto R^{\prime}=R \cap P^{\prime}$ whenever $\Pi(R)=P$ and $P^{\prime} \prec P$ (which entails a shift of rank by one), and the inclusions

$$
\overline{e^{\prime}(P)} \supset \overline{e^{\prime}(R)} \longleftrightarrow e^{\prime}\left(R^{\prime}\right) \longleftrightarrow \overline{e^{\prime}\left(P^{\prime}\right)},
$$

This is the same situation that was faced in (3.5.11), so $d_{1}$ is the composition of a restriction mapping and a connecting homomorphism. In the topological setting, the most efficient way to formulate this is without the Kostant decompositions, though the latter may be needed for calculations. We obtain the following formula:

Proposition 4.4.19. - When $P$ is conjugate to $P_{\rho+p}$ and $P^{\prime}$ is conjugate to $P_{\rho+p+1}$ and satisfies $P^{\prime} \prec P$,

$$
d_{1}: E_{1}^{p, q}\left(\bar{K}^{\bullet}, \mathcal{R}^{h}\right) \longrightarrow E_{1}^{p+1, q}\left(\bar{K}^{\bullet}, \mathcal{R}^{h}\right)
$$

in the spectral sequence for $\mathcal{R}^{h}$, is the direct sum of maps $\widetilde{E}_{1}^{p, q}(P) \rightarrow \widetilde{E}_{1}^{p+1, q}\left(P^{\prime}\right)$ given by

$$
H_{\ell, c}^{i}\left(e^{\prime}(P), \widetilde{\boldsymbol{E}}\right) \stackrel{r}{\longrightarrow} H_{\ell, c}^{i}\left(e^{\prime}\left(P \cap P^{\prime}\right), \widetilde{\boldsymbol{E}}\right) \stackrel{\delta}{\longrightarrow} H_{\ell, c}^{i+1}\left(e^{\prime}\left(P^{\prime}\right), \widetilde{\boldsymbol{E}}\right),
$$

where $r$ is restriction and $\delta$ a connecting homomorphism.

We quickly compare the nerve and holomorphic rank spectral sequences (see (4.4.6) and (4.4.10) resp.) in the least complicated non-trivial situation, namely when the $\mathbb{Q}$-rank of $G$ equals two. In that case, one has standard parabolics, maximal: $P_{1} \succ$ $P_{2}$, and minimal $P_{12}=P_{1} \cap P_{2}$. Both spectral sequences have only one non-trivial differential, so they both degenerate at $E_{2}$. For the sake of simplicity of notation, we will omit the symbol for summation over $\Gamma$-conjugacy classes in both cases, though it is important not to lose sight of this; said summation is explicit only in our formula 
for the terms of the holomorphic rank spectral sequence, but it is present in the other one as well.

The respective differentials $d_{1}$ look like:

$$
\begin{gathered}
H^{q}\left(\overline{e^{\prime}\left(P_{1}\right)}, \widetilde{\boldsymbol{E}}\right) \quad \stackrel{r}{\longrightarrow} H^{q}\left(e^{\prime}\left(P_{12}\right), \widetilde{\boldsymbol{E}}\right) \\
H^{q}\left(\frac{\oplus}{e^{\prime}\left(P_{2}\right)}, \widetilde{\boldsymbol{E}}\right) \stackrel{r}{\longrightarrow} H^{q}\left(e^{\prime}\left(P_{12}\right), \widetilde{\boldsymbol{E}}\right) \\
H_{\ell, c}^{q}\left(e^{\prime}\left(P_{1}\right), \widetilde{\boldsymbol{E}}\right) \stackrel{r}{\longrightarrow} H_{\ell, c}^{q}\left(e^{\prime}\left(P_{12}\right), \widetilde{\boldsymbol{E}}\right) \stackrel{\delta}{\longrightarrow} H_{\ell, c}^{q+1}\left(e^{\prime}\left(P_{2}\right), \widetilde{\boldsymbol{E}}\right) .
\end{gathered}
$$

The arithmetic quotients for $G_{\ell, P_{1}}$ and $G_{h, P_{2}}$ are compact; the nilmanifolds coming from the unipotent radicals are always compact. Thus, (4.4.20.h) simplifies to:

$$
\begin{aligned}
H^{q}\left(e^{\prime}\left(P_{1}\right), \widetilde{\boldsymbol{E}}\right) \stackrel{r}{\longrightarrow} & H^{q}\left(e^{\prime}\left(P_{12}\right), \widetilde{\boldsymbol{E}}\right) \stackrel{\delta}{\longrightarrow} \\
& H_{c}^{q+1}\left(e^{\prime}\left(P_{2}\right), \widetilde{\boldsymbol{E}}\right) \simeq H^{q+1}\left(\overline{e^{\prime}\left(P_{2}\right)}, \partial \overline{e^{\prime}\left(P_{2}\right)} ; \widetilde{\boldsymbol{E}}\right) .
\end{aligned}
$$

Since $\mathcal{R}$ is finer than $\mathcal{R}^{h}$, we have

$$
E_{2}^{0, q}(\bar{K}, \mathcal{R}) \rightarrow E_{2}^{0, q}\left(\bar{K}, \mathcal{R}^{h}\right), \quad E_{2}^{1, q}(\bar{K}, \mathcal{R}) \longleftrightarrow E_{2}^{1, q}\left(\bar{K}, \mathcal{R}^{h}\right)
$$

(these relations can also be deduced directly from (4.4.20.N) and (4.4.21.h)). By reason of degree, the spectral sequence degenerates at $E_{2}$ - this need not be true in higher rank - so the total dimension of $E_{2}$ in each degree is the same for both.

\subsection{The mixed Hodge complex filtered by holomorphic rank}

Given the isomorphism of deleted neighborhood cohomology for $\partial \bar{M}$ and $\partial M_{\Sigma}$ provided by $[\mathrm{HZ} 2,(3.5 .5)]$, we begin by replacing $\bar{K}^{\bullet}$ from (4.4.4) by any compatible system of cohomological mixed Hodge complexes (e.g., the de Rham complexes of the corresponding mixed Hodge modules), and put

$$
\widetilde{K}^{\bullet}(R)=C^{\bullet}\left(i_{R}^{*} \widetilde{\boldsymbol{E}}\right) .
$$

We will see that the double complex $\widetilde{K}^{\bullet}$, with its Hodge and weight filtrations (one can see $F$ via (4.1) here), is a mixed Hodge complex filtered by $\mathcal{R}^{h}$. The last notion is a technical condition, determined by El Zein, that implies that the spectral sequence for $\mathcal{R}^{h}$ is one of mixed Hodge structures. What the latter means is spelled out after [HZ2, (5.5.2)], and it includes the assertion that the differentials of the spectral sequence are morphisms of mixed Hodge structure. A useful criterion for that, which is easy to check in practice, is that $\mathcal{R}^{h}$ be a convolutant of the weight filtration $W$, which in turn implies that $\mathcal{R}^{h}$ splits on $\mathrm{Gr}^{W} \widetilde{K}^{\bullet}$ (see $[\mathrm{Z3},(3.6)]$ ).

We already know that $\mathcal{R}$ is a convolutant of $W$, with $W=\mathcal{R} * W^{H}$, where $W^{H}$ is the usual Hodge weight filtration on the individual summands of the complex. We 
also have that $\mathcal{R}=\mathcal{R}^{h} * \mathcal{R}^{\ell}$ by (3.5.9). However, this fact in general is not enough to give

$$
W=R^{h} *\left(R^{\ell} * W^{H}\right) ;
$$

one would need to check that the three filtrations $W^{H}, R^{h}$ and $R^{\ell}$ form a distributive family in the sense of [Ka, 1.7]. In this case, however, it is evident that (4.5.1) holds, and we leave it to the reader to verify this. We therefore assert:

Proposition 4.5.2. - The complex $\widetilde{K}^{\bullet}$ is a mixed Hodge complex filtered by $\mathcal{R}^{h}$ (as well as by $\mathcal{R}$ ) that determines the mixed Hodge structure of $\partial \bar{M}$ with coefficients in $\widetilde{\boldsymbol{E}}$.

For emphasis, we restate a consequence of (4.5.2) that was mentioned above:

COROLlary 4.5.3. - The holomorphic rank spectral sequence in the topological setting is a spectral sequence of mixed Hodge structures.

With (4.5.3) established, we make a Hodge theoretic comparison of the $E_{1}$-terms of the [topological] nerve and holomorphic rank spectral sequences. For that, we examine the respective formulas, (4.4.8) and (4.4.10.1), for the $E_{1}$-term. One thing stands out: they both involve the same variations of Hodge structure on $M_{P}$, viz., those coming from the representation of $G_{h, P}$ on the $E_{\mu(h, w)}$ 's. Where they differ is in the finite-dimensional vector spaces (with the trivial Hodge structure) that the cohomology groups $H^{a}\left(M_{P}, \widetilde{\boldsymbol{E}}_{\mu(h, w)}\right)$ are tensored with. As in [Z2], an "undesirable" term in a formula can disappear because it gets tensored with 0 .

Finally, we assert the conclusion towards which we have been heading:

THEOREM 4.5.4. - The holomorphic rank (resp. nerve) spectral sequence for coherent cohomology abutting to $H^{\bullet}\left(Z_{\Sigma}, i^{*} \operatorname{Gr}_{F}^{p}\left\{\mathcal{D} \mathcal{R}\left(M, \widetilde{\boldsymbol{E}}^{\text {can }}\right\}\right)\right.$ is canonically isomorphic to the $\mathrm{Gr}_{F}^{p}$ of the topological holomorphic rank (resp. nerve) spectral sequence abutting to $H_{\bullet}(\partial \bar{M}, \widetilde{\boldsymbol{E}}) \simeq H_{d n}^{\bullet}\left(Z_{\Sigma}, \widetilde{\boldsymbol{E}}\right)$.

REMARK 4.5.5. - The same holds for all $F^{p} / F^{p^{\prime}}$ (with $p^{\prime}>p$ ).

\subsection{Ghost classes}

We set up the question of the existence of ghosts in its natural settings. The reader should expect by now that there are two parallel notions, one for the topological setting (local systems) and one for the coherent setting (vector bundles).

Let $Y$ be a manifold-with-corners. Topologically, it is just a manifold-withboundary [BS, App.], but the boundary has designated differentiable corner structure. Let $\left\{E_{\alpha}\right\}$ denote the set of closed faces of codimension one in $\partial Y$, and let $\widetilde{\boldsymbol{V}}$ be a local system on $Y$. We mention that $Y$ and its interior are homotopically indistinguishable. The faces of $\partial Y$ are themselves manifolds-with-corners. If we define the $\operatorname{rank} \rho(Y)$ of $Y$ to be the largest number of maximal (codimension-one) faces of $\partial Y$ having 
non-empty intersection, or equivalently, the highest codimension of a boundary face, then, a face $E$ of codimension $m$ in $\partial Y$ has rank at most $\rho(Y)-m$, with equality if and only if $E$ contains a face of highest codimension in $\partial Y$. Note that this notion of rank is consistent with the intrinsic rank of parabolic subgroups when $Y=\bar{M}$.

A ghost class in $Y$ with coefficients in $\widetilde{\boldsymbol{V}}$ is an element in $H^{\bullet}(Y, \widetilde{\boldsymbol{V}})$ with a non-zero image in

$$
\begin{aligned}
G h^{\bullet}(Y, \tilde{\boldsymbol{V}}) & =\frac{\operatorname{Ker}\left\{H^{\bullet}(Y, \tilde{\boldsymbol{V}}) \rightarrow \bigoplus_{\alpha} H^{\bullet}\left(E_{\alpha}, \tilde{\boldsymbol{V}}\right)\right\}}{\operatorname{Ker}\left\{H \bullet(Y, \widetilde{\boldsymbol{V}}) \rightarrow H^{\bullet}(\partial Y, \widetilde{\boldsymbol{V}})\right\}} \\
& =\frac{\operatorname{Ker}\left\{H^{\bullet}(Y, \widetilde{\boldsymbol{V}}) \rightarrow \bigoplus_{\alpha} H^{\bullet}\left(E_{\alpha}, \widetilde{\boldsymbol{V}}\right)\right\}}{\operatorname{Im}\{H \bullet(Y, \partial Y ; \widetilde{\boldsymbol{V}}) \rightarrow H \bullet(Y, \widetilde{\boldsymbol{V}})\}}
\end{aligned}
$$

(it is denoted $\operatorname{Spect}^{\bullet}(Y, \widetilde{\boldsymbol{V}})$ in $\left.[\mathrm{Z} 5]\right)$. The above is isomorphic to its image under restriction to $\partial Y$ :

$$
\operatorname{Im}\left\{H^{\bullet}(Y, \tilde{\boldsymbol{V}}) \longrightarrow H^{\bullet}(\partial Y, \tilde{\boldsymbol{V}})\right\} \cap \operatorname{Ker}\left\{H^{\bullet}(\partial Y, \widetilde{\boldsymbol{V}}) \longrightarrow \bigoplus_{\alpha} H^{\bullet}\left(E_{\alpha}, \tilde{\boldsymbol{V}}\right)\right\}
$$

We call $G h \cdot(Y, \tilde{\boldsymbol{V}})$ the ghost group of $(Y, \tilde{\boldsymbol{V}})$. Note that the definition depends on the corner structure of $Y$. In the case of $Y=\bar{M}$, we have

$$
G h^{\bullet}(\bar{M}, \widetilde{\boldsymbol{E}})=\frac{\operatorname{Ker}\left\{H^{\bullet}(\bar{M}, \widetilde{\boldsymbol{E}}) \rightarrow \underset{P}{\bigoplus_{P}} H^{\bullet}\left(\overline{e^{\prime}(P)}, \widetilde{\boldsymbol{E}}\right)\right\}}{\operatorname{Im}\left\{H^{\bullet}(\bar{M}, \partial \bar{M} ; \widetilde{\boldsymbol{E}}) \rightarrow H^{\bullet}(\bar{M}, \widetilde{\boldsymbol{E}})\right\}},
$$

where $P$ runs over maximal parabolics.

Next, let $Y$ be instead a complex manifold, and $D$ an SNC divisor on $Y$. Let $\mathcal{F}$ be a locally-free sheaf on $Y$. We assume given a partition of the set of irreducible components of $D$, which decomposes $D$ into a union $\bigcup_{i} D_{i}$ (cf. (2.3)). Then a ghost class in $Y$ with coefficients in $\mathcal{F}$, relative to $\left\{D_{i}\right\}$, is an element of $H^{\bullet}(Y, \mathcal{F})$ that has non-zero image in

$$
G h^{\bullet}\left(Y,\left\{D_{i}\right\} ; \mathcal{F}\right)=\frac{\operatorname{Ker}\left\{H^{\bullet}(Y, \mathcal{F}) \rightarrow \bigoplus_{i} H^{\bullet}\left(D_{i}, \mathcal{F} \otimes \mathcal{O}_{D_{i}}\right)\right\}}{\operatorname{Ker}\left\{H \bullet(Y, \mathcal{F}) \rightarrow H \bullet\left(D, \mathcal{F} \otimes \mathcal{O}_{D}\right)\right\}},
$$

or equivalently:

$$
\begin{aligned}
\operatorname{Im}\left\{H^{\bullet}(Y, \mathcal{F}) \longrightarrow H^{\bullet}\left(D, \mathcal{F} \otimes \mathcal{O}_{D}\right)\right\} \cap \operatorname{Ker}\left\{H^{\bullet}\right. & \left(D, \mathcal{F} \otimes \mathcal{O}_{D}\right) \\
& \left.\longrightarrow \bigoplus_{i} H^{\bullet}\left(D_{i}, \mathcal{F} \otimes \mathcal{O}_{D_{i}}\right)\right\}
\end{aligned}
$$

Of course, we are most interested in the case where $Y=M_{\Sigma}$ is a toroidal compactification of a connected component of a Shimura variety, $i$ stands for $P$, and $D_{i}=Z_{\Sigma}(P)$; in that case, (4.6.4) becomes:

$$
G h^{\bullet}\left(M_{\Sigma},\left\{Z_{\Sigma}(P)\right\} ; \mathcal{F}\right)=\frac{\operatorname{Ker}\left\{H \bullet\left(M_{\Sigma}, \mathcal{F}\right) \rightarrow \bigoplus_{P} H \bullet\left(Z_{\Sigma}(P), \mathcal{F} \otimes \mathcal{O}_{Z_{\Sigma}(P)}\right)\right\}}{\operatorname{Ker}\left\{H \bullet\left(M_{\Sigma}, \mathcal{F}\right) \rightarrow H \bullet\left(Z_{\Sigma}, \mathcal{F} \otimes \mathcal{O}_{Z_{\Sigma}}\right)\right\}}
$$


The following is an immediate consequence of what we have developed in (4.1.7) and $[\mathrm{HZ} 2,(5.6)]$ :

Proposition 4.6.7. - There is a natural decomposition

$$
\operatorname{Gr}_{F}^{\bullet} G h^{\bullet}(\bar{M}, \widetilde{\boldsymbol{E}}) \simeq G h^{\bullet}\left(M_{\Sigma},\left\{Z_{\Sigma}(P)\right\} ; \operatorname{Gr}_{F}^{\bullet} \mathcal{D} \mathcal{R}(M, \widetilde{\boldsymbol{E}})^{\mathrm{can}}\right) .
$$

REMARK 4.6.8. - Contained in the above assertion is the fact that the Hodge components of a ghost class are themselves ghost classes. It follows that the existence of ghost classes for the topological cohomology imply the existence of the same for at least one of the vector bundles on the right-hand side and conversely.

Though we could state the essential content of the Hodge-theoretic criterion (4.6.14) now, we first elect to formulate (4.6.3) in terms of a single filtered mixed Hodge complex. Let

$$
\overline{\mathfrak{C}^{\bullet}}=\operatorname{Cone}\left\{C^{\bullet}(\bar{M}, \widetilde{\boldsymbol{E}}) \longrightarrow C^{\bullet}(\partial \bar{M}, \widetilde{\boldsymbol{E}})\right\}[-1],
$$

and $\widetilde{\mathfrak{C}}$ its Hodge-theoretic quasi-isomorph:

$$
\widetilde{\mathfrak{C}}^{\bullet}=\operatorname{Cone}\left\{C^{\bullet}\left(M_{\Sigma}, R j_{*} \widetilde{\boldsymbol{E}}\right) \longrightarrow C_{\mathrm{dn}}^{\bullet}\left(\partial M_{\Sigma}, \widetilde{\boldsymbol{E}}\right)\right\}[-1] .
$$

We have

$$
\left.H^{\bullet}\left(\widetilde{\mathfrak{C}^{\bullet}}\right) \simeq H^{\bullet}\left(\overline{\mathfrak{C}^{\bullet}}\right) \simeq H^{\bullet}(\bar{M}, \partial \bar{M} ; \widetilde{\boldsymbol{E}})\right) .
$$

As the cone of a morphism of mixed Hodge complexes, (4.6.10) is a mixed Hodge complex in the standard way (see [E, p. 76]). We extend the simplicial filtration from the boundary complex by simply setting $\widetilde{\mathfrak{C}^{\bullet}}=S_{1}$, and likewise for $\overline{\mathfrak{C}^{\bullet}}$. We now assert:

Proposition 4.6.12. - In terms of the filtered mixed Hodge complex $\left(\widetilde{\mathfrak{C}}^{\bullet}, S\right)$, the ghost group $G h^{\bullet}(\bar{M}, \widetilde{\boldsymbol{E}})$ equals

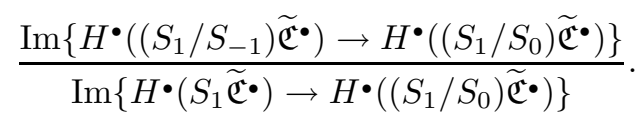

Of course, we may replace $\widetilde{\mathfrak{C}}^{\bullet}$ in the above by $\overline{\mathfrak{C} \bullet}$.

The "denominator" in (4.6.12) (see (4.6.3)) is, in degree $i$,

$$
\operatorname{Im}\left\{H_{c}^{i}(M, \widetilde{\boldsymbol{E}}) \longrightarrow H^{i}(M, \widetilde{\boldsymbol{E}})\right\} .
$$

For $E$ an irreducible representation of $G$ of weight $e$, the weights occuring in the left-hand side are $\leqslant i+e$, and those occurring in the right-hand side are $\geqslant i+e$. It is immediate, then, that the mixed Hodge structure of (4.6.13) is actually pure of weight $i+e$. We obtain the following criterion for the existence of ghosts.

Criterion 4.6.14. - Assume that $\widetilde{\boldsymbol{E}}$ is pure of weight e. If

$$
\operatorname{Im}\left\{H^{i}\left(\left(S_{1} / S_{-1}\right) \widetilde{\mathfrak{C}^{\bullet}}\right) \longrightarrow H^{i}\left(\left(S_{1} / S_{0}\right) \widetilde{\mathfrak{C}^{\bullet}}\right)\right\} \simeq \operatorname{Ker}\left\{H^{\bullet}(\bar{M}, \widetilde{\boldsymbol{E}}) \rightarrow \bigoplus_{P} H^{\bullet}\left(\overline{e^{\prime}(P)}, \widetilde{\boldsymbol{E}}\right)\right\}
$$

is not pure of weight $i+e$, then there are ghost classes in $H^{i}(\bar{M}, \widetilde{\boldsymbol{E}})$. 
REMARK. - Of course, (4.6.14) makes no statement about the possibility that the weight $i+e$ summand of $G h^{i}(\bar{M}, \widetilde{\boldsymbol{E}})$ be non-zero. The method of [KR] finds ghosts for $\widetilde{\boldsymbol{E}}=\mathbb{C}(e=0)$ that are in the image of the Borel map (i.e., among the cohomology classes of the invariant forms). These are of weight $i$. Thus, the above criterion is independent of theirs.

There are other ways to write the ghost group, and each emphasizes a different Hodge-theoretic relation. From the point of view in (4.6.2), we have the equivalent formulas:

$$
\begin{aligned}
&(4.6 .15)(\mathrm{i}) \quad G h^{\bullet}(\bar{M}, \widetilde{\boldsymbol{E}}) \simeq \operatorname{Ker}\left\{H^{\bullet}\left(S_{0} \widetilde{\mathfrak{C}}^{\bullet}\right) \rightarrow H^{\bullet}\left(S_{1} \widetilde{\mathfrak{C}}^{\bullet}\right) \oplus H^{\bullet}\left(\left(S_{0} / S_{-1}\right) \widetilde{\mathfrak{C}^{\bullet}}\right)\right\} \\
& \simeq \operatorname{Ker}\left\{H^{\bullet}(\partial \bar{M}, \widetilde{\boldsymbol{E}}) \rightarrow\left(H^{\bullet}(\bar{M}, \partial \bar{M} ; \widetilde{\boldsymbol{E}})[1] \oplus \bigoplus_{P} H^{\bullet}\left(e^{\prime}(P), \widetilde{\boldsymbol{E}}\right)\right)\right\} \\
&(4.6 .15)(\mathrm{ii}) \quad G h^{\bullet}(\bar{M}, \widetilde{\boldsymbol{E}}) \\
& \simeq \operatorname{Ker}\left\{H^{\bullet}\left(S_{0} \widetilde{\mathfrak{C}^{\bullet}}\right) \rightarrow H^{\bullet}\left(S_{1} \widetilde{\mathfrak{C}^{\bullet}}\right)\right\} \cap \operatorname{Im}\left\{H^{\bullet}\left(S_{-1} \widetilde{\mathfrak{C}}^{\bullet}\right) \rightarrow H^{\bullet}\left(S_{0} \widetilde{\mathfrak{C}}^{\bullet}\right)\right\} \\
& \simeq \operatorname{Ker}\left\{H^{\bullet}(\partial \bar{M}, \widetilde{\boldsymbol{E}}) \rightarrow H^{\bullet}(\bar{M}, \partial \bar{M} ; \widetilde{\boldsymbol{E}})[1]\right\} \cap \operatorname{Im}\left\{H^{\bullet}\left(S_{-1} \widetilde{\mathfrak{C}}^{\bullet}\right) \rightarrow H^{\bullet}(\partial \bar{M}, \widetilde{\boldsymbol{E}})\right\} .
\end{aligned}
$$

The apparent difference between (4.6.15) and (4.6.14) is that the former has one looking at

$$
\begin{aligned}
H^{\bullet}(\partial \bar{M}, \widetilde{\boldsymbol{E}}) \longrightarrow \bigoplus_{P} H^{\bullet} & \left(e^{\prime}(P), \widetilde{\boldsymbol{E}}\right) \\
& \simeq \underset{P \sim P_{1}}{\bigoplus} H^{\bullet}\left(M_{P}, \widetilde{\boldsymbol{H}} \cdot\left(\mathfrak{w}_{P}, E\right)\right) \oplus \underset{P \sim P_{2}}{\bigoplus} H^{\bullet}\left(\mathfrak{w}_{P}, E\right),
\end{aligned}
$$

whereas (4.6.14) emphasizes

$$
H^{\bullet}(\bar{M}, \widetilde{\boldsymbol{E}}) \longrightarrow \bigoplus_{P} H^{\bullet}\left(e^{\prime}(P), \widetilde{\boldsymbol{E}}\right)
$$

REMARK 4.6.18. - The cohomology $H^{i}\left(S_{-1} \widetilde{\mathfrak{C}}^{\bullet}\right)$ is that of the codimension-two skeleton of $\partial \bar{M}$, viz.,

$$
H^{i-1}\left(\bigcup_{r(Q)=2} \overline{e^{\prime}(Q)}, \widetilde{\boldsymbol{E}}\right) .
$$

The above union is a non-vacuous disjoint union if and only if $G$ is of $\mathbb{Q}$-rank two, in which case it equals

$$
\bigoplus_{r(Q)=2} H^{i-1}\left(e^{\prime}(Q), \widetilde{\boldsymbol{E}}\right)
$$

In effect, the method used in [Z5, App. A] to rule out ghosts for $G=G S p(4)$ $(4 \times 4$ matrices), was based on (4.6.15)(ii). The main step was to examine the weight structure of $H^{\bullet}\left(S_{-1} \widetilde{\mathfrak{C}^{\bullet}}\right) / \operatorname{Im} H^{\bullet}\left(S_{0} / S_{-1} \widetilde{\mathfrak{C}^{\bullet}}\right)$, viewing $H^{\bullet}\left(S_{-1} \widetilde{\mathfrak{C}^{\bullet}}\right)$ as the iterated deleted neighborhood cohomology associated to $e^{\prime}(Q) \subset \overline{e^{\prime}(P)} \subset M$ for $P$ of rank one and containing $Q$. Note that when $G=G S p(4), M_{P}$ is a modular curve (uncompactified) if $P \sim P_{1}$ and is a point if $P \sim P_{2}$. 
The local system $\widetilde{\boldsymbol{E}}$ on $M$ gets its weights from the weight homomorphism $w_{G}$, whereas the local systems on $M_{P}$ get theirs from $w_{P}$. The two are related by the formula $w_{P}=m_{P} \cdot w_{G}$ (see [HZ1, (1.2.2.1)]); $m_{P}$ gives rise to the so-called parabolic weights, and the latter are computed from Kostant's theorem.

4.6.A. Appendix: The case of $G S p_{4}$. - For arithmetic quotients $X$ associated to a reductive group $G$, with $\operatorname{rk}_{\mathbb{Q}} G^{\text {ad }}=2$, the ghost group can be approached via (4.6.15)(ii) and (4.6.18). It is not so difficult to see what is at stake, and we describe it here, following and correcting [Z5, App. A].

The maximal closed faces of $\partial \bar{X}$ consist of the Borel-Serre compactifications of arithmetic quotients of spaces associated to $\mathbb{Q}$-rank one groups. These are of two types, corresponding to the two conjugacy types, $P_{1}$ and $P_{2}$, of maximal parabolic subgroups of $G$; the two types of closed boundary face are here denoted $\bar{X}_{1}$ and $\bar{X}_{2}$, and their respective interiors $X_{1}$ and $X_{2}$. An intersection of type $\bar{X}_{1} \cap \bar{X}_{2}$ is a space of type $X_{12}$, corresponding to the minimal parabolics $P_{12}$. The unipotent radicals of the parabolics give rise to proper nilmanifold fibrations, e.g., $X_{1} \rightarrow \widehat{X}_{1}$, so the cohomology of $X_{1}$ (with coefficients) gets expressed as cohomology with coefficients in Lie algebra cohomology; likewise for $X_{2} \rightarrow \widehat{X}_{2}$ and $X_{12} \rightarrow \widehat{X}_{12}$. The numbering has been chosen so that when $X$ is a connected Shimura variety, $\widehat{X}_{1}$ is a connected Shimura variety of a $\mathbb{Q}$-rank one group, and $\widehat{X}_{2}$ is collapsed in the map to the BailyBorel (cf. (4.4)).

The geometry of the boundary is well-known (see the appendix to [HZ2, (3.5)]):

Proposition 4.6.A.1. - Any two closed faces of type $X_{1}$ are disjoint, as are two of type $X_{2}$ or $X_{12}$. The boundary of a face of type $X_{1}$ or $X_{2}$ consists of a finite number of faces of type $X_{12}$, and each face of type $X_{12}$ is a boundary face of exactly one face of type $X_{1}$, and of exactly one face of type $X_{2}$.

Let

$$
\begin{aligned}
& P G h^{i}(X, \widetilde{\boldsymbol{E}}) \\
& \quad=\operatorname{Coker}\left\{\bigoplus H^{i-1}\left(X_{1}, \widetilde{\boldsymbol{E}}\right) \oplus \bigoplus H^{i-1}\left(X_{2}, \widetilde{\boldsymbol{E}}\right) \rightarrow \bigoplus H^{i-1}\left(X_{12}, \widetilde{\boldsymbol{E}}\right)\right\}
\end{aligned}
$$

where the sums are over the set of faces of the given type, be the pre-ghost group; it is only from here that ghost classes could arise. When we decompose the cohomology groups in (4.6.A.2) according to Kostant (cf. (4.6.16)), we get the direct sum of mappings of the form

$$
\begin{aligned}
& H^{k}\left(\widehat{X}_{1}, \widetilde{\boldsymbol{E}}_{w^{1}}\right) \longrightarrow H_{\mathrm{dn}}^{k}\left(\widehat{X}_{12}, \widetilde{\boldsymbol{E}}_{w^{1}}\right) \\
& H^{k}\left(\widehat{X}_{2}, \widetilde{\boldsymbol{E}}_{w^{2}}\right) \longrightarrow H_{\mathrm{dn}}^{k}\left(\widehat{X}_{12}, \widetilde{\boldsymbol{E}}_{w^{2}}\right),
\end{aligned}
$$

in which $w^{j} \in W^{j}$ is a Kostant representative. The mappings (4.6.A.3) give the means for killing potential pre-ghosts. 
In the case of $G S p(4)$, everything is at its easiest. The space $\widehat{X}_{1}$ and $\widehat{X}_{2}$ are both modular for $G L(2)$, and $\widehat{X}_{12}$ is just a point, reducing us to $k=0,1$ in (4.6.A.3). It turns out that $k=1$ is the more interesting, and we denote by $P G h^{i}(X, \widetilde{\boldsymbol{E}})_{1}$ the corresponding subspace of $P G h^{i}(X, \widetilde{\boldsymbol{E}})$. (We refer the reader to [Z5] to see that ghost classes cannot come from $k=0$.) Since $\widehat{X}_{1}$ and $\widehat{X}_{2}$ are both non-compact orientable surfaces - call them $S$ - the mappings in (4.6.A.3), when summed over the boundary circles of a single $S$, can be interpreted as the natural mapping

$$
H^{1}(S, \widetilde{\boldsymbol{E}}) \longrightarrow H^{0}\left(\partial S^{*}, R^{1} j_{*} \widetilde{\boldsymbol{E}}\right),
$$

where $j: S \hookrightarrow S^{*}$ is the inclusion of $S$ in its compactification with cusps. For simple topological reasons, (4.6.A.4) is surjective if and only if $\widetilde{\boldsymbol{E}}$ is a non-trivial local system. When $\widetilde{\boldsymbol{E}} \simeq \mathbb{C}$, we have instead

$$
\operatorname{dim} \operatorname{Coker}\left\{H^{1}(S, \widetilde{\boldsymbol{E}}) \longrightarrow H^{0}\left(\partial S^{*}, R^{1} j_{*} \widetilde{\boldsymbol{E}}\right)\right\}=1 .
$$

On the other hand, $H^{0}(S, \widetilde{\boldsymbol{E}}) \rightarrow H^{0}\left(\partial S^{*}, R^{0} j_{*} \widetilde{\boldsymbol{E}}\right)$ is never surjective.

Let $\lambda_{1}$ and $\lambda_{2}$ be the fundamental dominant weights for the $C_{2}$ root system. Let $E=E_{\lambda}$, and write $\lambda=m_{1} \lambda_{1}+m_{2} \lambda_{2}$.

Calculation 4.6.A.6 ([Z5, (A.1.16)])

(i) $E_{w_{1}}$ is trivial as a representation of $S L(2)$ if and only if $l\left(w^{1}\right)=0$ or 3 , and $m_{2}=0$

(ii) Similarly, $E_{w_{2}}$ is trivial as a representation of $S L(2)$ if and only if $l\left(w^{2}\right)=0$ or 3 , and $m_{1}=0$.

We rewrite the right-hand side of (4.6.A.3) as
(i) $E_{\tau_{1} w^{1}}$
(ii) $E_{\tau_{2} w^{2}}$,

where $\tau_{j}$ is the non-trivial element of the $A_{1}$ Weyl group $W_{j}$ of $P_{j}$. This enables us to invoke (4.6.A.1) and write down a basis of $P G h^{\bullet}\left(X, \widetilde{\boldsymbol{E}}_{\lambda}\right)_{1}$ :

Proposition 4.6.A.8. - Let $w^{12}$ be an element of the Weyl group $W^{12}$ of $C_{2}$, and $E_{w^{12}}$ the corresponding vector space. Then: $P G h^{\bullet}\left(X, \widetilde{\boldsymbol{E}}_{\lambda}\right)_{1}$ is generated by the $E_{w^{12}}$, with $w^{12}$ satisfying one of: $w^{12}=\tau_{1} w^{1}, l\left(w^{1}\right)=0,3$, and $m_{2}=0$; or $w^{12}=\tau_{2} w^{2}$, $l\left(w^{2}\right)=0,3$, and $m_{1}=0$.

It is useful to keep in mind that $\tau_{1} W^{1} \bigcup \tau_{2} W^{2}$ excludes just the identity element of $W^{12}$. Similarly, $\tau_{1} W^{1} \cap \tau_{2} W^{2}$ is the longest element $(l=4)$ of $W^{12}$. We conclude:

Corollary 4.6.A.9. - The exceptions to the assertion "PGh $\left(X, \widetilde{\boldsymbol{E}}_{\lambda}\right)_{1}=0$ " are given by:

(i) $i=2: l\left(w^{1}\right)=0$, and $m_{2}=0$; or $l\left(w^{2}\right)=0$, and $m_{1}=0$,

(ii) $i=5: l\left(w^{1}\right)=3$ or $l\left(w^{2}\right)=3$, and $m_{1}=m_{2}=0$.

We prove next: 
Proposition 4.6.A.10. $-P G h^{2}\left(X, \widetilde{\boldsymbol{E}}_{\lambda}\right)_{1} \cap G h^{2}\left(X, \widetilde{\boldsymbol{E}}_{\lambda}\right)=0$ unless $\left(m_{1}, m_{2}\right)=$ $(0,0)$.

By means of the other calculations in [Z5:(A.1)], we conclude:

Corollary 4.6.A.11. $-G h^{2}\left(X, \widetilde{\boldsymbol{E}}_{\lambda}\right)=0$ unless $\left(m_{1}, m_{2}\right)=(0,0)$.

Proof of (4.6.A.10). - Let $w_{\lambda}$ denote the (Hodge) weight of $\widetilde{\boldsymbol{E}}_{\lambda}$. It is actually defined only up to an even integer, and the choice will be seen to be irrelevant.

We recall the tables from [Z5:(A.1.15)], in abbreviated form, in which the parabolic weights are determined from Kostant's theorem:

$$
\begin{aligned}
& \begin{array}{llll}
W^{1} & \underline{\ell} \quad \frac{\text { parabolic weight }}{m_{1}+m_{2}} & \frac{\text { highest weight for } S L(2)}{m_{2}} \\
0 &
\end{array} \\
& 3 \quad-m_{1}-m_{2}-4 \quad m_{2} \\
& \begin{array}{llll}
W^{2} & \underline{\ell} & \frac{\text { parabolic weight }}{m_{1}+2 m_{2}} & \frac{\text { highest weight for } S L(2)}{m_{1}}
\end{array} \\
& 3-m_{1}-2 m_{2}-6 \quad m_{1}
\end{aligned}
$$

Again, to get a trivial local system at the boundary, we must have either $m_{2}=0$ (for $1 \in W^{1}$ ) or $m_{1}=0$ (for $1 \in W^{2}$ ). This gives, for the corresponding trivial local systems on $\widehat{X}_{1}$ and $\widehat{X}_{2}$, parabolic weights $m_{1}$ and $2 m_{2}$ resp. It follows that $H^{1}\left(X_{12}, \widetilde{\boldsymbol{E}}_{\lambda}\right)$ is of Hodge theoretic weights $w_{\lambda}-m_{1}+2$ and $w_{\lambda}-2 m_{2}+2$ resp. We must check whether this can match a weight occurring in $H^{2}\left(X, \widetilde{\boldsymbol{E}}_{\lambda}\right)$. The latter are all $\geqslant 2+w_{\lambda}$, which is higher than those of $H^{1}\left(X_{12}, \widetilde{\boldsymbol{E}}_{\lambda}\right)$ except when $m_{1}=m_{2}=0$. Our assertion is proved.

\section{REMARK}

(i) The only possibility for a term $E_{w^{12}}$ entering into $P G h^{i}\left(X, \widetilde{\boldsymbol{E}}_{\lambda}\right)$ that is not covered by (4.6.A.9) is for $w^{12}=1$ (when $i=1$ ). This does not yield a non-zero element of $G h^{i}\left(X, \widetilde{\boldsymbol{E}}_{\lambda}\right)$, for the weights are wrong ([Z5, (A.1.18)]).

(ii) The ghost class constructed in $[\mathrm{KR}]$ is for $i=2$ and $m_{1}=m_{2}=0$. 



\section{CHAPTER 5}

\section{ON THE COMPARISON OF HODGE STRUCTURES}

It has been more than ten years since the "Zucker Conjecture" (see $[\mathrm{Z} 2, \S 6]$ ) was proved. We quickly recall the statement in (5.1) below. Let $M^{*}$ be the Baily-Borel Satake compactification of a connected component $M$ of a Shimura variety.

TheORem 5.1 ([L], $[\mathrm{SS}])$. - Let $G$ be the group in the Shimura datum giving rise to $M, E$ a rational representation space for $G$, and $\widetilde{\boldsymbol{E}}$ the associated local system on $M$. Then there is a quasi-isomorphism

$$
\mathcal{L}_{(2)}^{\bullet}\left(M^{*}, \widetilde{\boldsymbol{E}}\right) \approx \mathcal{I C} \bullet\left(M^{*}, \widetilde{\boldsymbol{E}}\right),
$$

between the sheaves of $L^{2} \widetilde{\boldsymbol{E}}$-valued differential forms and the $\widetilde{\boldsymbol{E}}$-valued intersection cochains on $M^{*}$.

This has the standard consequence:

Corollary 5.2. - For all $k, H_{(2)}^{k}(M, \widetilde{\boldsymbol{E}}) \simeq I H^{k}\left(M^{*}, \widetilde{\boldsymbol{E}}\right)$.

Upon seeing (5.2), it is natural to expect more. Recall that each side of the isomorphism comes with an associated Hodge structure. For the $L^{2}$-cohomology (the left-hand side), it comes from the $L^{2}$ harmonic forms (see [SZ]); for the intersection cohomology, it comes via Morihiko Saito's Hodge modules [Sa1]. A priori, the two Hodge structures need not correspond under the isomorphism. However, there is an inevitable conjecture, the Hodge theoretic version of (5.2):

Conjecture 5.3. - The isomorphism in (5.2) is an isomorphism of Hodge structures.

This conjecture remains unresolved. A few cases of (5.3), in which $M^{*}$ has only isolated singular points, are covered in [Z4]. We remind the reader that one cannot even be sure a priori that the Hodge numbers $\left(\operatorname{dim} H^{p, q}\right)$ coincide.

In the direction of (5.3), we now make the following improvement on [H5, 3.3.9]: 
Theorem 5.4. - For all $k$, the mapping

$$
r_{k}: H_{(2)}^{k}(M, \widetilde{\boldsymbol{E}}) \simeq I H^{k}\left(M^{*}, \widetilde{\boldsymbol{E}}\right) \longrightarrow H^{k}(M, \widetilde{\boldsymbol{E}})
$$

is morphism of mixed Hodge structures.

\section{REMARKS 5.5}

(i) The image of $r_{k}$ is the lowest non-zero weight level in the mixed Hodge structure of $H^{k}(M, \widetilde{\boldsymbol{E}})$. This can be deduced from the decomposition theorem: $I H^{k}\left(M^{*}, \widetilde{\boldsymbol{E}}\right)$ is seen to have the same image in $H^{k}(M, \widetilde{\boldsymbol{E}})$ as $I H^{k}\left(M_{\Sigma}, \widetilde{\boldsymbol{E}}\right)$ has, and the latter determines the lowest non-zero weight level. Thus, (5.4) asserts that the Hodge structures in (5.2) have a given common Hodge-theoretic quotient. By semi-simplicity, this quotient can be embedded as a common substructure.

(ii) Let $c$ denote the codimension of the singular locus in $M^{*}$. Included in (5.2) is the assertion that $r_{k}$ is an isomorphism whenever $k<c$, and is injective for $k=c$. Thus, from (5.4) it follows that (5.3) is true in degrees $k \leqslant c$.

We will be referring to the following commutative diagram:

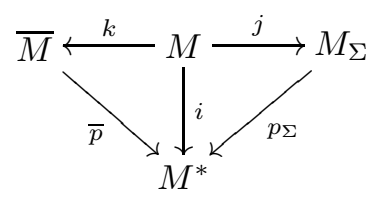

As in (4.1.8), let $\mathcal{D} \mathcal{R}(M, \widetilde{\boldsymbol{E}})$ be the holomorphic de Rham complex of $M$ with values in $\widetilde{\boldsymbol{E}}$. We have the explicit formula on $M_{\Sigma}$ :

$$
\mathcal{D R}(M, \widetilde{\boldsymbol{E}})^{\mathrm{can}}=\Omega_{M_{\Sigma}}\left(\log Z_{\Sigma}\right) \otimes \mathcal{E}^{\mathrm{can}},
$$

a complex quasi-isomorphic to $R j_{*} \widetilde{\boldsymbol{E}}$. We write $\mathcal{A} \cdot\left(\mathcal{D R}(M, \widetilde{\boldsymbol{E}})^{\text {can }}\right)$ to denote the Dolbeault resolution of (5.7); this (or more precisely, its associated single complex) is also quasi-isomorphic to $R j_{*} \widetilde{\boldsymbol{E}}$. We also let

$$
A^{\bullet}=A^{\bullet}\left(\mathcal{D R}(M, \widetilde{\boldsymbol{E}})^{\mathrm{can}}\right)
$$

denote the complex of its global sections. Then $H^{\bullet}\left(A^{\bullet}\right)=H^{\bullet}(M, \widetilde{\boldsymbol{E}})$.

It is clear that $A^{\bullet}$ is a subcomplex of

$$
A_{\mathrm{sia}}^{\bullet}=A_{\mathrm{sia}}^{\bullet}(M, \widetilde{\boldsymbol{E}}),
$$

the global sections of $\mathcal{A}_{\text {sia }}^{\bullet}\left(M_{\Sigma}, Z_{\Sigma}\right) \otimes \Omega_{M_{\Sigma}}^{\bullet}\left(\log Z_{\Sigma}\right) \otimes \mathcal{E}^{\text {can }}$, which is equivalently (by (2.6.1)) the global sections of the de Rham complex of forms with moderate growth $\mathcal{A}_{\text {sia }}^{\bullet}(\bar{M}, \widetilde{\boldsymbol{E}})$ on $\bar{M}$. We know that $\mathcal{A}_{\text {sia }}^{\bullet}(\bar{M}, \widetilde{\boldsymbol{E}})$ is quasi-isomorphic to $R k_{*} \widetilde{\boldsymbol{E}}[\mathrm{B} 2,7.4]$. There is a tautological extension of $\widetilde{\boldsymbol{E}}$ to a local system on $\bar{M}$, as the latter is a manifold-with-corners, and $R k_{*} \widetilde{\boldsymbol{E}}$ is quasi-isomorphic to that. Thus, $H^{\bullet}\left(A_{\mathrm{sia}}^{\bullet}\right)$ is also $H \bullet(M, \widetilde{\boldsymbol{E}})$, so the inclusion of $A^{\bullet}$ in $A_{\text {sia }}^{\bullet}$ is a quasi-isomorphism. 
Both $A^{\bullet}$ and $A_{\text {sia }}^{\bullet}$ inherit a filtration $F$ from the usual Hodge filtration on $A^{\bullet}(M, \widetilde{\boldsymbol{E}})$, which is given in (4.1.2). Note that $\left(A^{\bullet}, F\right)$ underlies the standard mixed Hodge complex for $H^{\bullet}(M, \widetilde{\boldsymbol{E}})$.

Proposition 5.8. - The inclusion of filtered complexes $\left(A^{\bullet}, F\right) \hookrightarrow\left(A_{\text {sia }}, F\right)$ is a filtered quasi-isomorphism.

Proof. - We must show that for all $p$,

$$
\operatorname{Gr}_{F}^{p} A^{\bullet} \longrightarrow \operatorname{Gr}_{F}^{p} A_{\text {sia }}^{\bullet}
$$

is a quasi-isomorphism. Because of the way the filtration $F$ is given, we can rewrite (5.8.1) as

$$
A^{\bullet}\left\{\operatorname{Gr}_{F}^{p}\left(\mathcal{D} \mathcal{R}(M, \widetilde{\boldsymbol{E}})^{\mathrm{can}}\right)\right\} \longrightarrow A_{\text {sia }}^{\bullet}\left\{\operatorname{Gr}_{F}^{p}\left(\mathcal{D} \mathcal{R}(M, \widetilde{\boldsymbol{E}})^{\mathrm{can}}\right)\right\} .
$$

Now, as we mentioned in $\S 4, \operatorname{Gr}_{F}^{p}\left(\mathcal{D} \mathcal{R}(M, \widetilde{\boldsymbol{E}})^{\text {can }}\right)$ is a complex of $\mathcal{O}_{M_{\Sigma}}$-modules. That (5.8.2) is a quasi-isomorphism follows by applying (2.2.5) to each term. ${ }^{(1)}$

Corollary 5.9. - The filtration $F$ on $A_{\text {sia }}^{\bullet}$ induces the Hodge filtration on $H^{\bullet}(M, \widetilde{\boldsymbol{E}})$.

We can now prove (5.4). Let $\mathfrak{h}^{k}$ denote the space of $\widetilde{\boldsymbol{E}}$-valued harmonic $k$-forms on $M$. According to $[\mathrm{BG}], \mathfrak{h}^{k}$ consists of forms of moderate growth. Then, the inclusion

$$
\mathfrak{h}^{\bullet} \longleftrightarrow A_{\text {sia }}^{\bullet}(M, \widetilde{\boldsymbol{E}})
$$

which is for trivial reasons compatible with $F$, induces the morphisms $r_{k}$. We may invoke (5.5)(i) and the strictness principle for Hodge structures: a morphism of filtered vector spaces $(H, F) \rightarrow\left(H^{\prime}, F^{\prime}\right)$ in which $F$ and $F^{\prime}$ define Hodge structures is a morphism of Hodge structures. This completes the proof of (5.4).

\footnotetext{
(1) Alternatively, it is possible to invoke (4.1.12) at this point.
} 



\section{BIBLIOGRAPHY}

[AMRT] Ash, A., Mumford, D., Rapoport, M., Tai, Y.-S., Smooth compactification of Locally Symmetric Varieties, Math. Sci. Press, Brookline, MA, 1975.

[BHR] Blasius, D., Harris, M., Ramakrishnan, D., Coherent cohomology, limits of discrete series, and Galois conjugation, Duke Math. J., 73, 647-685 (1994).

[B1] Borel, A., Introduction to automorphic forms, PSPM 9, In: Algebraic Groups and Discontinuous Subgroups, Proc. Symp. Pure Math. AMS, 9, 199-210 (1966).

[B2] Borel, A., Stable real cohomology of arithmetic groups, Ann. Scient. Ec. Norm. Sup., 7, 235-272 (1974).

[B3] Borel, A., Introduction aux Groupes Arithmétiques, Hermann, Paris, 1969.

[BG] Borel, A., Garland, H., Laplacian and the discrete spectrum of an arithmetic group, Am. J. Math., 105, 309-335 (1983).

[BS] Borel, A., Serre, J.-P., Corners and arithmetic groups. Comm. Math. Helv., 48, 436-491 (1973).

[De1] Deligne, P., Equations différentielles à points singuliers réguliers. Lecture Notes in Math., 163, (1970).

[De2] Deligne, P., Théorie de Hodge, III. Publ. Math. IHES 44, 5-77 (1974).

[E] El Zein, F., Mixed Hodge structures, Trans. AMS 275, 71-106 (1983).

[F] Faltings, G., On the cohomology of locally symmetric hermitian spaces, Lect. Notes in Math., 1029, 55-98 (1984).

[Fr1] Franke, J., Harmonic analysis in weighted $L_{2}$ spaces, Ann. Sci. Ec. Norm. Sup., 31, 181-279 (1998).

[Fr2] Franke, J., A topological model for some summand in the Eisenstein cohomology of congruence subgroups (appended to $[\mathrm{KR}]$ ). 
[GH] Griffiths, P., Harris, J., Principles of Algebraic Geometry, John Wiley and Sons, New York (1978).

[Ha] Harder, G., On the cohomology of discrete arithmetically defined groups. In: Proceedings of the International Colloquium on Discrete Subgroups of Lie Groups and Applications to Moduli. Oxford Univ. Press, Bombay, 1975, 129-160.

[H1] Harris, M., Arithmetic vector bundles and automorphic forms on Shimura varieties. I. Invent. Math., 82, 151-189 (1985); II, Compositio Math., 60, 323-378 (1986).

[H2] Harris, M., Functorial properties of toroidal compactifications of locally symmetric varieties, Proc. Lon. Math. Soc. 59, 1-22 (1989).

[H3] Harris, M., Automorphic forms and the cohomology of vector bundles on Shimura varieties, in L. Clozel and J.S. Milne, eds., Proceedings of the Conference on Automorphic Forms, Shimura Varieties, and $L$-functions, Ann Arbor, 1988, Perspectives in Mathematics, New York: Academic Press, Vol. II, 41-91 (1989).

[H4] Harris, M., Automorphic forms of $\bar{\partial}$-cohomology type as coherent cohomology classes, J. Diff. Geom., 32, 1-63 (1990)

[H5] Harris, M., Hodge-de Rham structures and periods of automorphic forms. In: Motives (Seattle, 1991), Proc. Symp. Pure Math. AMS, 55, Part 2, pp. 573-624 (1994).

[HP] Harris, M., Phong, D.H., Cohomologie de Dolbeault à croissance logarithmique à l'infini. C. R. Acad. Sci. Paris, 302, 307-310 (1986).

[HZ1] Harris, M., Zucker, S.: Boundary cohomology of Shimura varieties, I: coherent cohomology on the toroidal boundary, Ann. Scient. Ec. Norm. Sup., 27, 249-344 (1994).

[HZ2] Harris, M., Zucker, S.: Boundary cohomology of Shimura varieties, II: Hodge theory at the boundary, Inventiones Math., 116, 243-307 (1994); Erratum, Inventiones Math., 121, p. 437 (1995).

[Ka] Kashiwara, M.: The asymptotic behavior of a variation of polarized Hodge structure, Publ. RIMS Kyoto Univ., 21 (1985), 853-875.

[KKMS] Kempf, G., Knudsen, F., Mumford, D., Saint-Donat, B., Toroidal embeddings, Lecture Notes in Math., 339, (1973).

[KR] Kewenig, A., Rieband, Th.: Geisterklassen im Bild der Borelabbildung für symplektishe und orthogonale Gruppen, Diplomarbeit, Uni. Bonn, 1997.

[K] Kostant, B.: Lie algebra cohomology and the generalized Borel-Weil theorem, Ann. of Math., 74, 329-287 (1961). 
[Le] Lepowsky, J., A generalization of the Bernstein-Gelfand-Gelfand resolution, J. Algebra, 49, 496-511 (1977).

[L] Looijenga, E., $L_{2}$-cohomology of locally symmetric varieties, Compositio Math., 67, 3-20 (1988).

[M1] Milne, J.S., Automorphic vector bundles on connected Shimura varieties, Invent. Math., 92, 91-128 (1988).

[M2] Milne, J.S., Canonical models of (mixed) Shimura varieties and automorphic vector bundles. In: Clozel, L., Milne, J. (eds.), Automorphic Forms, Shimura Varieties, and L-functions, v. 1, 283-414 (1990).

[M3] Milne, J.S., Descent for Shimura varieties. Michigan Math. J., 46, 203-208 (1999).

[Mo] Moonen, B., Models of Shimura varieties in mixed characteristics, in A. J. Scholl and R. L. Taylor, eds., Galois Representations in Arithmetic Algebraic Geometry, London Mathematical Society Lecture Note Series, 254, 267-350 (1998).

$[\mathrm{Mu}]$ Mumford, D., Hirzebruch's proportionality theorem in the non-compact case, Inventiones Math., 42, 239-272 (1977).

[P] Pink, R., Arithmetical compactifications of mixed Shimura varieties. Bonn Math. Schrift., Nr. 209 (1990).

[RC] Rocha-Caridi, A., Splitting criteria for $\mathfrak{g}$-modules induced from a parabolic and the Bernstein-Gelfand-Gelfand resolution of a finite dimensional, irreducible $\mathfrak{g}$-module, Trans. Am. Math. Soc., 262, 335-366 (1980).

[Sa1] Saito, Mo., Modules de Hodge polarisables. Publ. RIMS Kyoto Univ., 24 (1988), 849-995.

[Sa2] Saito, Mo.: Mixed Hodge modules and admissible variations. C.R.A.S. Paris, 309, 351-356 (1989).

[Sa3] Saito, Mo., Mixed Hodge modules, Publ. RIMS Kyoto Univ. 26, 221-333 (1990).

[SS] Saper, L., Stern M., $L_{2}$-cohomology of arithmetic varieties. Ann. of Math. 132 (1990), 1-69.

[SZ] Saper, L., Zucker, S., An introduction to $L^{2}$-cohomology. Several Complex Variables and Complex Geometry, Santa Cruz, 1989 Proc. of Symposia in Pure Math. 52 (1991), Part 2, 519-534.

[Sch] Schwermer, J., Kohomologie arithmetisch definierter Gruppen und Eisensteinreihen, Lecture Notes in Math., 988, (1983). 
BIBLIOGRAPHY

[SGA 3] Demazure, M., Grothendieck, A., Schémas en groupes, Lecture Notes in Math., 151, 152, 153 (1970).

[StZ] Steenbrink, J., Zucker, S., Variation of mixed Hodge structure, I. Inventiones Math., 80, 489-542, (1985).

[W1] Wildeshaus, J., Realizations of polylogarithms, Lecture Notes in Math., 1650, (1997).

[W2] Wildeshaus, J., Mixed sheaves on Shimura varieties and their higher direct images in toroidal compactifications, J. Alg. Geom. 9 (2000), 323-353.

[WK] Wolf, J. Kóranyi, A., Generalized Cayley transformations of bounded symmetric domains, Amer. J. Math., 87, 899-939 (1965).

[Z1] Zucker, S., Locally homogeneous variations of Hodge structure. L'Enseignement Math. 27, 243-275, (1981).

[Z2] Zucker, S., $L_{2}$ cohomology of warped products and arithmetic groups. Inventiones Math. 70, 169-218, (1982).

[Z3] Zucker, S., Degeneration of mixed Hodge structures. Algebraic Geometry, Proc. of Symposia in Pure Math., 46, 283-293, (1987).

[Z4] Zucker, S., The Hodge structures on the intersection homology of varieties with isolated singularities. Duke Math. J. 55, (1987), 603-616.

[Z5] Zucker, S., On the boundary cohomology of locally symmetric varieties. Vietnam J. Math. 25, 279-318, (1997).

[Z6] Zucker, S., $L_{2}$ cohomology and intersection homology of locally symmetric varieties, II. Compositio Math. 59, (1986), 339-398. 